\title{
Advances in theory and their application within the field of zeolite chemistry
}

\author{
Veronique Van Speybroeck ${ }^{a}$, Karen Hemelsoet ${ }^{a}$, Lennart Joos ${ }^{a}$, Michel Waroquier ${ }^{a}$, \\ Robert G Bell ${ }^{b}$ and C Richard A Catlow ${ }^{b}$
}

\author{
${ }^{a}$ Center for Molecular Modeling, Ghent University, \\ Technologiepark 903, 9052 Zwijnaarde, Belgium \\ ${ }^{b}$ Department of Chemistry, University College London, \\ 20 Gordon St., London WC1, United Kingdom
}

Corresponding authors : veronique.vanspeybroeck@ugent.be, c.r.a.catlow@ucl.ac.uk

Zeolites are versatile and fascinating materials which are vital for a wide range of industries, due to their unique structural and chemical properties, which are the basis of applications in gas separation, ion exchange and catalysis. Given their economic impact, there is a powerful incentive for smart design of new materials with enhanced functionalities to obtain the best material for a given application. Over the last decades, theoretical modeling has matured to a level that model guided design has become within reach. Major hurdles have been overcome to reach this point and almost all contemporary methods in computational materials chemistry are actively used in the field of modeling zeolite chemistry and applications. Integration of complementary modeling approaches is necessary to obtain reliable predictions and rationalizations from theory. A close synergy between experimentalists and theoreticians has led to a deep understanding of the complexity of the system at hand, but also allowed the identification of shortcomings in current theoretical approaches. Inspired by the importance of zeolite characterization which can now be performed at the single atom and single molecule level from experiment, computational spectroscopy has grown in importance in the last decade. In this review most of the currently available modeling tools are introduced and illustrated on the most challenging problems in zeolite science. Directions for future model developments will be given. 
Table of Contents Entry

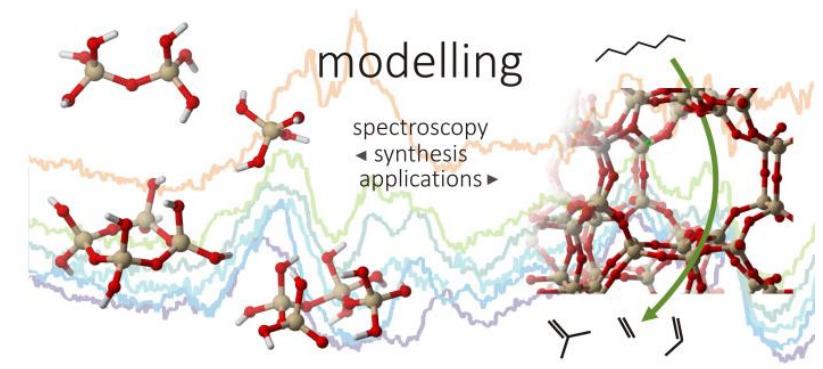

Advances in theory and their application in the field of zeolite chemistry and their rich applications are reviewed. The role of a broad range of theoretical methods to address the most challenging problems within zeolite science is highlighted. 

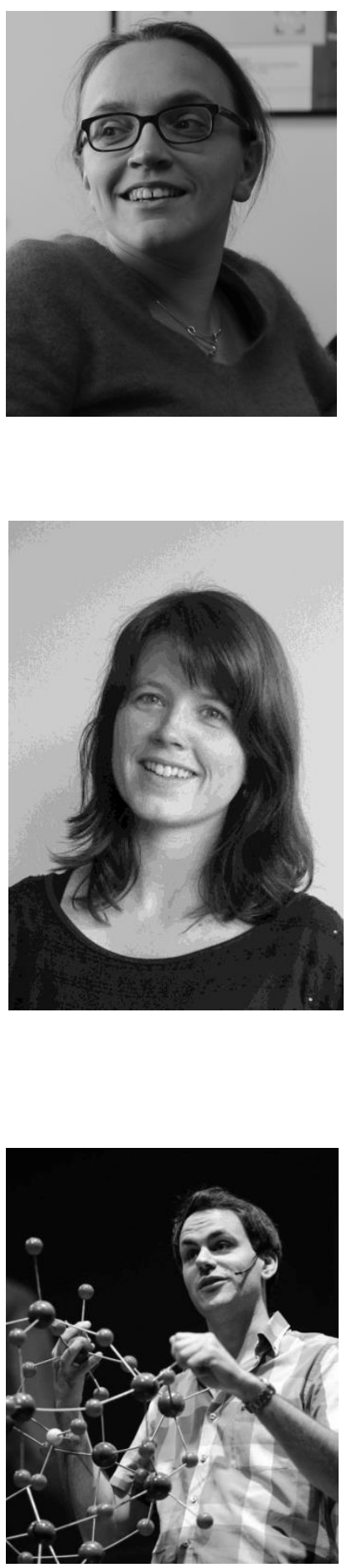

Veronique Van Speybroeck is full professor at the Ghent University and head of the Center for Molecular Modeling . She obtained her Ph.D in 2001 from the Ghent University. She built up a large expertise in first principle kinetics in nanoporous materials in the frame of an ERC starting grant, awarded in 2009. Her current research focusses on first principle molecular dynamics simulations of complex chemical transformation in nanoporous materials, for which she received an ERC Consolidator grant in 2015. She is an elected member of the Royal (Flemish) Academy for Science and the Arts of Belgium.

Karen Hemelsoet is assistant professor at Ghent University (Belgium). She graduated as an Engineer in Physics in 2002 and obtained her PhD in 2007. During her PhD she studied reactivity indicators and coke species using first-principle simulations. She stayed one year in the Inorganic Chemistry and Catalysis Group (Utrecht University, The Netherlands) of Prof. B. Weckhuysen, examining absorbance spectroscopy involving zeolites. Present research interests relate to computational spectroscopy, focusing on vibrational and electronic excitations. The main goal is the combination of these techniques for understanding the structure/property relations of systems, such as flexible dyes and catalytic materials.

Lennart Joos started his PhD at the Center for Molecular Modeling in 2011 under the supervision of Prof. Van Speybroeck, in close collaboration with the experimental group of Prof. Kirschhock and Martens at the KU Leuven. His research interests include zeolite formation, properties and applications such as carbon capture. In view of the latter topic, he stayed for a year in the group of Prof. Berend Smit at the University of California, Berkeley on a Fulbright scholarship. 


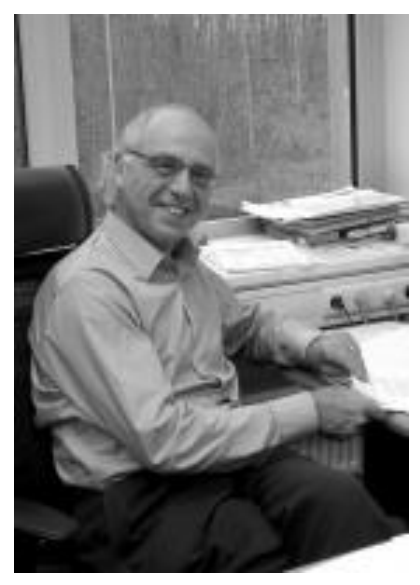

Michel Waroquier is emeritus full professor in physics at the Ghent University since 2012. In 1997 he founded the Center for Molecular Modeling (CMM) together with Professor Veronique Van Speybroeck. He succeeded in turning his former research field around, and in building a team of more than 30 people, bringing together physicists and chemists. The various research areas cover advanced methodologies for catalyst design, for the prediction of chemical kinetics of reactions catalyzed over nanoporous materials, and for the computation of spectroscopic properties (EPR,NMR, IR, Raman).

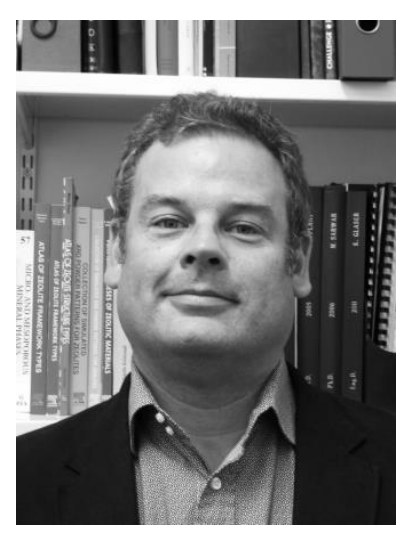

Robert Bell received his BSc from University College London (UCL) and PhD from the University of Southampton. He spent over 15 years researching at the Royal Institution of Great Britain and is now is Reader in Computational Chemistry at UCL. His research covers the application of modelling methods to many current problems in materials chemistry. These include sorption and diffusion of gases in zeolites, MOFs and ionic liquids, including modelling of $\mathrm{CO}_{2}$ capture and separation; Catalysis in zeolitic systems and structure prediction of microporous materials.

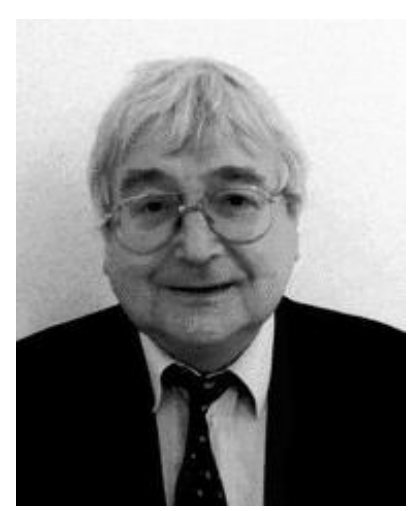

Richard Catlow obtained his doctorate from The University of Oxford and subsequently held academic appointments at University College London, Keele University, The Daresbury Laboratory and The Royal Institution. He is currently Professor of Materials Chemistry at UCL. His research interests are in the field of the chemistry of complex materials, including those for applications in catalytic and energy applications, investigated by a combination of computational and experimental techniques. 


\section{Introduction}

Zeolites are amongst the most widely investigated and topical of inorganic materials. Their synthesis, structures and properties pose fascinating fundamental problems, while their wide-ranging industrial applications have stimulated both the optimization of existing zeolitic structures and the search for new materials with new functionalities. Computational techniques have proved to be indispensable in progressing the field of zeolite-based technologies and in the pursuit of a molecular based design of zeolite-based materials for specific applications. Enormous progress has been made within the field of computational chemistry and materials design, which may be ascribed to development of novel and ingenious methods and algorithms combined with the steady and rapid increase in computer power. Modelling and simulation of zeolites makes use both of all the tools available in contemporary computational materials chemistry and of the full range of hardware from desk-top PC to massively parallel High Performance computing (HPC) resources. This review will illustrate the role of these methods in addressing many of the most challenging problems in zeolite science; and we will show how modelling tools have acquired a predictive character which is increasingly exploited in applied zeolite science.

Like the whole area of computational modeling also the field of theory and zeolites, has benefited largely from evolutions in HPC resources. This together with major methodological advances resulted in the fact that systems of growing realism may be simulated with increasing accuracy. The interested reader is referred to a special issue dedicated to HPC computing in the chemistry of materials that appeared in 2014 and was edited by Woodley and Catlow. ${ }^{1}$ Furthermore the perspective written by de Jong et al. ${ }^{2}$ on the status of parallel computing in quantum chemistry, nicely emphasizes that both advances in hardware, software and methods are closely intertwined and necessary to address larger and more complex systems. ${ }^{1}$

The next section will introduce some of the major industrial applications of zeolites; but the pervasive nature of these applications is worth emphasizing from the outset. For example, every drop of gasoline has seen the inner structure of several zeolites. The catalytic properties and specific architecture of these nanoporous materials are able to transform and purify molecules from crude oil into a fuel that meets technical and environmental requirements for cars. Other petrochemical products are cracked, isomerized and/or hydrotreated inside zeolites. Almost every packet of detergent contains zeolites, exploiting the exceptional ion-exchange properties of these materials. Because of their porous structure and interaction sites, zeolites also have a large potential for gas adsorption. Carbon Capture and Sequestration, methane adsorption and hydrogen storage are only 
a few of the possible applications. The combination of adsorption and catalytic properties is optimally exploited in the selective catalytic reduction of $\mathrm{NO}_{x}$ by ammonia that converts the hazardous $\mathrm{NO}_{x}$ into harmless $\mathrm{N}_{2}$. Zeolites are also able to tackle catalytic challenges in future biorefineries. Their framework has the necessary active sites and can further be functionalized by impregnation with metal particles. However, as the molecules in renewable feedstock are larger and contain more heteromolecules than their fossil counterparts, zeolites with large pores and high stability will be needed. ${ }^{3,4}$ Furthermore we also want to point out the exciting evolutions found in the field of zeolitic membranes, providing new opportunities for separation, coatings, $\mathrm{CO}_{2}$ capture,... This topic is however outside the scope of this review but the interested reader is pointed to interested references in this area. ${ }^{5-7}$

Computational techniques are now applied to almost all aspects of zeolite science. They may be used routinely to model and increasingly to predict zeolite structures. The complex field of zeolite synthesis, including nucleation, growth and dissolution is being increasingly illuminated by the applications of modelling methods. Modelling has been applied with great success to many systems within the huge field of catalysis effected by zeolites; and modelling tools are indispensable aids in the study of molecular sorption and diffusion. All these applications will be addressed in the current review which will emphasise the role of modelling as a tool to be used in direct conjunction with experiment.

The field is now very extensive and our review is necessarily selective; we refer the reader to references for further information on zeolite science and the role of modelling methods. ${ }^{8,9}$ Given the extensive scope of this review, a plethora of modeling techniques will be touched upon, which are often categorized according to various length and time scales on which studied phenomena are occurring. An ingenious coupling from one scale to the other is required to understand the overall complexity of the macroscopic behavior. Indeed the synthesis of a zeolite and the observed function of the material is the result of a multi-scale nature of various phenomena, as was illustrated by Weckhuysen et al. in his reviews on spatial heterogeneities in catalytic solids at different length and time scales. ${ }^{10,11}$ Dedicated reviews on multiscale modeling techniques are readily available. ${ }^{12-}$ 14 In this review the various methods will be introduced throughout the text on a case by case basis, showing their applicability for a given problem. The interested reader is referred to topical textbooks and reference works on each of the different modeling domains. However to assist the reader, a summarizing table has been introduced with the various computational and theoretical methods discussed in this review and their application to various problems in zeolite science. (Table 1.1) 
Table 1.1 : Computational and theoretical methods discussed in this review and their application to various problems in zeolite science.

\begin{tabular}{|c|c|c|}
\hline \multicolumn{2}{|c|}{20} & computational techniques \\
\hline \multicolumn{3}{|c|}{ 3. Zeolite synthesis } \\
\hline \multicolumn{2}{|c|}{ 3.1 Early stages of zeolite growth } & \\
\hline & Condensation of small silica clusters in gas phase & Molecular Mechanics ; DFT \\
\hline & $\begin{array}{l}\text { Condensation/Oligomerization of small silica and } \\
\text { alumino-silicate clusters + water/complex } \\
\text { molecular environment }\end{array}$ & $\begin{array}{l}\text { DFT ; DFT/COSMO; DFT/ explicit water molecules; MP2; ab- } \\
\text { initio MD ; Transition Interface Sampling ; kinetic MC }\end{array}$ \\
\hline \multicolumn{3}{|c|}{ 3.2 Surface processes and crystal growth } \\
\hline & Surface structure and crystal growth & Periodic DFT ; MC \\
\hline \multicolumn{3}{|c|}{ 3.3 Template design } \\
\hline & Template-host interactions & $\begin{array}{l}\text { Minimization techniques/Interatomic Potentials; DFT ; } \\
\text { classical MD }\end{array}$ \\
\hline & Template design / template \& structure direction & codes : Zebeddee and Zeobuilder \\
\hline \multicolumn{3}{|c|}{ 4. Structural Modeling } \\
\hline & Hypothetical zeolite structures & $\begin{array}{l}\text { Symmetry constrained intersite bonding search method } \\
\text { (SCIBS) ; simulated annealing (ZEFSA II) ; tiling theory with } \\
\text { structure optimization with force fields }\end{array}$ \\
\hline & Feasibility of known structures & $\begin{array}{l}\text { Selection criterion on basis of lattice enthalpy (FF with } \\
\text { GULP), degree of distortion of tetrahedra, local interatomic } \\
\text { distances (LIDs), flexibility using Rigid Unit model, Natural } \\
\text { Building Units (NBUs) }\end{array}$ \\
\hline & Conversion of existing zeolites & \\
\hline & germanosilicate UTL as layered zeolite & FF ; DFT ; periodic DFT for interlayer interactions \\
\hline & reversible interconversion of (-)COK-14 & $\begin{array}{l}\text { Cluster/periodic DFT; temperature corrections; classical } \\
\text { force fields for flexibility analysis }\end{array}$ \\
\hline & External surfaces of zeolites & Interatomic Potential Method \\
\hline \multicolumn{3}{|c|}{ 5. Spectroscopy } \\
\hline \multicolumn{2}{|c|}{5.1 Infrared spectroscopy } & $\begin{array}{l}\text { Normal mode analysis ; partial Hessian approaches ; MD } \\
\text { with FFs; }\end{array}$ \\
\hline & CO as probe molecule for acidity of zeolite & DFT on cluster and periodic models for CO adsorption \\
\hline & Scaling methods for frequencies & $\begin{array}{l}\text { large cluster models; periodic DFT ; MD with periodic DFT } \\
\text { codes (VASP) ; QM-pot ; } \omega / \mathrm{r} \text { correlation method }\end{array}$ \\
\hline \multicolumn{2}{|c|}{ 5.2 NMR spectroscopy } & $\begin{array}{l}\text { periodic DFT codes using CASTEP, GIPAW, VASP, } \\
\text { QUICKSTEP/CP2K }\end{array}$ \\
\hline & Aluminophosphates & multi-technique approach \\
\hline & Structure-spectrum relationships & DFT \\
\hline & Template synthesis of zeolites & DFT \\
\hline & Fluoride synthesis route for Si/Ge zeolites & cluster models ; periodic DFT \\
\hline \multicolumn{2}{|c|}{ 5.3 Optical spectroscopy } & TD-DFT ; large clusters models ; periodic models \\
\hline & UV/Vis & MD-averaged TD-DFT \\
\hline & dye molecules in zeolites & $\begin{array}{l}\text { Frozen Density Embedding theory (FDET) ; first principles } \\
\text { MD }\end{array}$ \\
\hline \multicolumn{3}{|c|}{ 6. Applications using zeolites } \\
\hline \multicolumn{3}{|c|}{$6.1 \mathrm{CO}_{2}$ capture } \\
\hline & gas separation $\left(\mathrm{CO}_{2} / \mathrm{N}_{2}\right.$ selectivity) & GCMC and FF simulations \\
\hline & Carbon Capture and Storage (CCS) & \\
\hline & $\begin{array}{l}\text { screening of materials : metrics for CCS } \\
\text { performance }\end{array}$ & $\begin{array}{l}\text { GCMC with FFs a la carte ; structural metrics (Zeo++, } \\
\text { ZEOMICS, Poreblazer, Topos,...); concept of natural tilings }\end{array}$ \\
\hline & $\begin{array}{l}\text { Henry coefficients, adsorption isotherms, } \\
\text { enthalpies }\end{array}$ & $\begin{array}{l}\text { FF development a la carte (for use in GCMC) from periodic } \\
\text { DFT-D }\end{array}$ \\
\hline $\begin{array}{l}6.2 \mathrm{Co} \\
\text { acids }\end{array}$ & nversion of hydrocarbons over zeolitic & \\
\hline
\end{tabular}




\begin{tabular}{|c|c|c|}
\hline \multicolumn{2}{|r|}{ alkane adsorption } & $\begin{array}{l}\text { FFs ; cluster models ; periodic DFT-D ; QM-pot ; ONIOM ; } \\
\text { MD with periodic DFT-D codes }\end{array}$ \\
\hline & dispersion & $\begin{array}{l}\text { Add-on Lennard Jones potential in periodic DFT ; DFT-D ; } \\
\text { contemporary DFT functionals ; MP2 + RPA in clusters ; } \\
\text { hybrid MP2/plane wave based DFT scheme in periodic code } \\
\text {; QM-pot }\end{array}$ \\
\hline \multicolumn{2}{|r|}{ alkene adsorption } & \\
\hline & \begin{tabular}{l|l} 
& $\begin{array}{l}\text { physisorption versus chemisorption } \\
\text { persistent carbenium ions }\end{array}$
\end{tabular} & $\begin{array}{l}\text { QM-pot (MP2//B3LYP); hybrid MP2/plane wave based DFT } \\
\text { scheme in periodic code ; ab-initio MD in periodic DFT-D } \\
\text { code; ONIOM; metadynamics }\end{array}$ \\
\hline \multicolumn{2}{|r|}{ alkane cracking } & cluster models ; ONIOM ; periodic DFT-D ; MC using FFs \\
\hline & \begin{tabular}{l|l} 
& active site coverage \\
\end{tabular} & ab initio MD \\
\hline & \begin{tabular}{l|l} 
& product selectivity, reactive pathways \\
\end{tabular} & QCT simulations ; transition path sampling (TPS) \\
\hline \multicolumn{2}{|r|}{ complex reaction routes in MTO } & \\
\hline & \begin{tabular}{l|l} 
& free energy profiles
\end{tabular} & $\begin{array}{l}\text { extended cluster models ; combined DFT and microkinetic } \\
\text { models, periodic DFT, ab initio MD }\end{array}$ \\
\hline & $\begin{array}{l}\text { Complexity with additional guest } \\
\text { molecules }\end{array}$ & metadynamics \\
\hline \multicolumn{2}{|c|}{ 6.3 Redox/Oxidation } & \\
\hline & $\begin{array}{l}\text { Hydrocarbon oxidation in microporous } \\
\text { aluminophosphates }\end{array}$ & periodic DFT (CRYSTAL) \\
\hline & Titanium substituted zeolites as redox catalysts & QM/MM (CHEMSHELL) \\
\hline & Metal exchanged zeolites for $\mathrm{NH}_{3}-\mathrm{SCR}$ & $\begin{array}{l}\text { extended cluster models ; periodic DFT ; first principles MD } \\
\text { (CP2K) }\end{array}$ \\
\hline \multicolumn{2}{|c|}{ 6.4 Diffusion } & MD with FFs (DL_POLY) ; Kinetic MC \\
\hline & Linear alkane diffusion & \\
\hline & Diffusion in confinement & \\
\hline
\end{tabular}

This review is structured as follows: In the next section we present a brief summary of zeolite structural science, synthesis and applications which will provide context for our subsequent account of the application of modelling techniques. Section 3 and 4 give a comprehensive review of modeling efforts used to unravel the zeolite formation processes, including zeolite surface modeling and structural modeling to guide processes towards desired structures for a given application.

Section 5 gives a current status on the field of computational spectroscopy, which has grown in importance tremendously in the last decade. This evolution has been triggered and inspired by major experimental progress in in-situ characterization techniques. Section 6 gives a flavor of the rich application field of zeolites, encompassing, $\mathrm{CO}_{2}$ capture, hydrocarbon conversion processes using both Brønsted acidic catalysts and redox/oxidation systems but also diffusion processes. Finally an outlook is given and expected future developments in the field of theory for zeolites. 


\section{Condensed overview of current zeolite-based materials and their applications}

\subsection{Structure and Synthesis}

Zeolites may be loosely defined as crystalline framework structured alumino-silicates with pores and cages of molecular dimensions; although in practice, several significant zeolitic materials, e.g. silicalite, are close to being pure silica polymorphs. The materials are tecto-silicates, built out of $\mathrm{SiO}_{4}$ and $\mathrm{AlO}_{4}$ tetrahedra linked into corner-sharing networks; and when aluminum is present in the framework, electro-neutrality requires the incorporation of charge compensating cations, which occupy extra-framework sites in the channels and cages. Almost any cationic species (alkali and alkaline earth, transition metal and lanthanide cations) can be included; and an important special case is compensation by protons which bond to the bridging oxygen atoms, thereby creating Brønsted acid sites (see Figure 2.1.1) which play a key role in much of zeolite catalysis.

Zeolites occur naturally as minerals, for example large deposits of clinoptilolite are found in several locations worldwide. However, most of the materials used in industrial applications are synthesised using hydrothermal procedures, in which an aqueous synthesis gel, at high $\mathrm{pH}$, containing sources of $\mathrm{Si}, \mathrm{Al}$ and appropriate cations is treated under moderate temperatures and pressure and the material slowly crystallises. A widely used procedure is to add an organic template, commonly an alkylammonium cation to the synthesis gel which "directs" the resulting structure towards specific framework architectures. Both natural and as-prepared synthetic zeolites are hydrated with large amounts of water occluded in their pores. Extra-framework cations are hydrated and located at loosely defined sites. For applications in catalysis and separations discussed below, dehydrated materials are necessary; water of hydration can easily be removed at modest temperatures (500 $600 \mathrm{~K}$ ). However, when a synthesis template is used, higher temperature calcination is needed to burn off the occluded organic matter. Fortunately, zeolite frameworks are robust and survive this treatment. Other post synthesis treatment may include steam de-alumination to increase the Si/Al ratio. The high degree of stability of the frameworks is kinetically and not thermodynamically based. Both experimental calorimetry and lattice energy calculations (as discussed later in this review) show that zeolites are metastable with respect to dense alumino-silicates and indeed, if raised to sufficiently high temperatures (1200-1300 K), the frameworks collapse into a dense polymorph. The whole field of zeolite synthesis and stability is a challenging one to which modelling methods have made a substantial contribution as will be discussed later in this review. 
(a) MFI

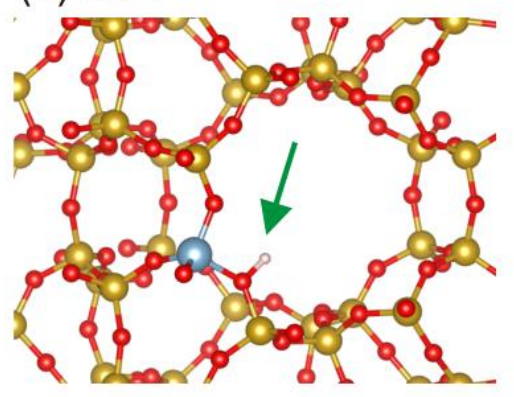

(c) BEA

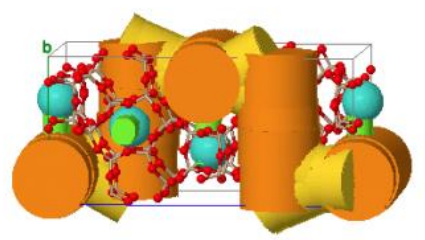

(f) $\mathrm{BCT}$

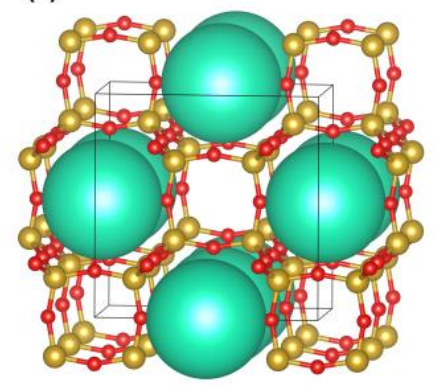

(b) FAU

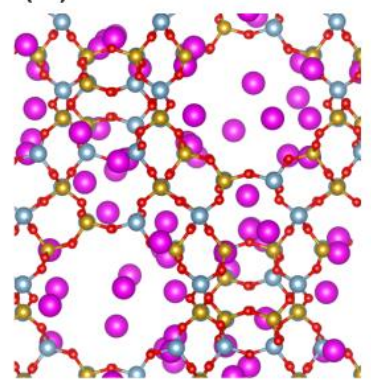

(e) FER

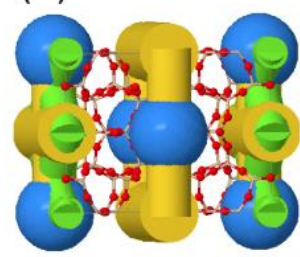

(g) TSC

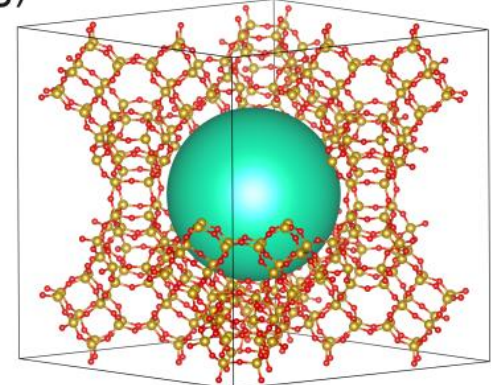

Figure 2.1.1 : Zeolite structures of the 'Big Five', the five zeolites that are most commonly used in industrial applications. Si is shown in yellow, Al in grey, $\mathrm{O}$ in red and $\mathrm{Na}$ in magenta. (a) H-ZSM-5, with the MFI topology and one Brønsted Acid site indicated with the green arrow (b) zeolite $\mathrm{Y}$, with the FAU topology and $\mathrm{Na}+$ countering the negative charges of $\mathrm{Al}$ in the framework (c) zeolite beta, with the BEA topology (d) mordenite (MOR) and (e) ferrierite (FER). The figures for the all-silica zeolites (c-e) are obtained with ZEOMICS. ${ }^{15-18}$ Channel and pore structure of the material are displayed. (f) BCT topology with small pores (cavity in the double four-ring has size $3.8 \AA$ ) (g) TSC structure with a very large pore of $16.4 \AA$.

Zeolites have a rich and intriguing structural chemistry, to whose understanding computational methods have made a substantial contribution, as discussed later. The most recent Atlas of Zeolite Framework Types lists 225 framework structures for microporous tetrahedral frameworks of all compositions. ${ }^{19}$ These materials are often classified according to their pore dimensions. The nomenclature small, medium and large pore zeolites are used for opening of the micropores 
constituted by 8,10 or 12 tetrahedra units in the ring. The pores have molecular dimensions and the possibility to tune the pore aperture lies on the basis for their usage as molecular sieves and shape selective behavior within catalysis. ${ }^{20}$ Some typical and important structures are illustrated in Figure 2.1.1. We note that in the dehydrated unlike the hydrated systems, the cations often occupy well defined sites in the cages or channels, but that in such sites they are typically undercoordinated, which is important in some applications.

The zeolite family may be extended in many ways. Metal atoms may be introduced into framework as well as extra-framework sites, although the level of such substitution is low, with the classic example being the replacement of $\sim 1 \%$ of Si by Titanium in the pure-silica material silicalite to give the "TS-1" oxidation catalysts to which we return later in this review. Both Ga and Ge may also be introduced into framework tetrahedral sites and we will consider later some of the significant new germano-silicate structures. A whole new class of structures of framework structured aluminaphosphates (ALPOs) was discovered in the $1980 \mathrm{~s}^{21}$ in which the building blocks are $\mathrm{AlO}_{4}$ and $\mathrm{PO}_{4}$ tetrahedra. Many of the structures are direct analogues of known zeolites, although some new structural types have been synthesised. The materials have an $\mathrm{Al} / \mathrm{P}$ ratio of very close to one and hence their frameworks are electro-neutral, without compensating cations. However, divalent cations (e.g. $\mathrm{Mg}$ ) can be substituted for $\mathrm{Al}$ and Si for $\mathrm{P}$ (generating "SAPOs") which require charge compensation, most importantly by protons generating acid sites. ALPO frameworks are less robust to heat treatment than zeolites, which has limited their applications. They do potentially have a substantial advantage over zeolites in the greater ease in which metal ions can be introduced into the frameworks; for example transition metal ions ( e.g. $\mathrm{Mn}, \mathrm{Fe}, \mathrm{Co}$ ) can be introduced at levels of 10\%) which leads to significant opportunities in oxidation catalysis as will be discussed later.

The broader class of framework materials with molecular dimensions is generally referred to as "microporous materials" of which zeolites and ALPOs can be considered as sub-classes. The whole family of porous silica and related materials was further broadened in the 1990s by the discovery of meso-porous materials, ${ }^{22,23}$ which have regular structures with pores of the order of 20-50.

Detailed discussion of these fascinating materials is beyond the scope of this article although we will refer to them subsequently.

Two final comments should be noted on zeolite structural chemistry. The first is the importance of their crystallinity, which is of significance in several applications and which also means that they are open to characterisation by the powerful range of both experimental and computational techniques that are available for investigating periodic systems. The second is that despite their crystallinity, they contain significant and in some case high levels of defects, both point and extended, which can play a vital role in controlling their properties. 


\subsection{Applications}

Many of the most significant and interesting challenges in zeolite science arise from their wide ranging applications in industry. All the applications, which are of very considerable economic importance, arise from their fundamental structural and chemical properties.
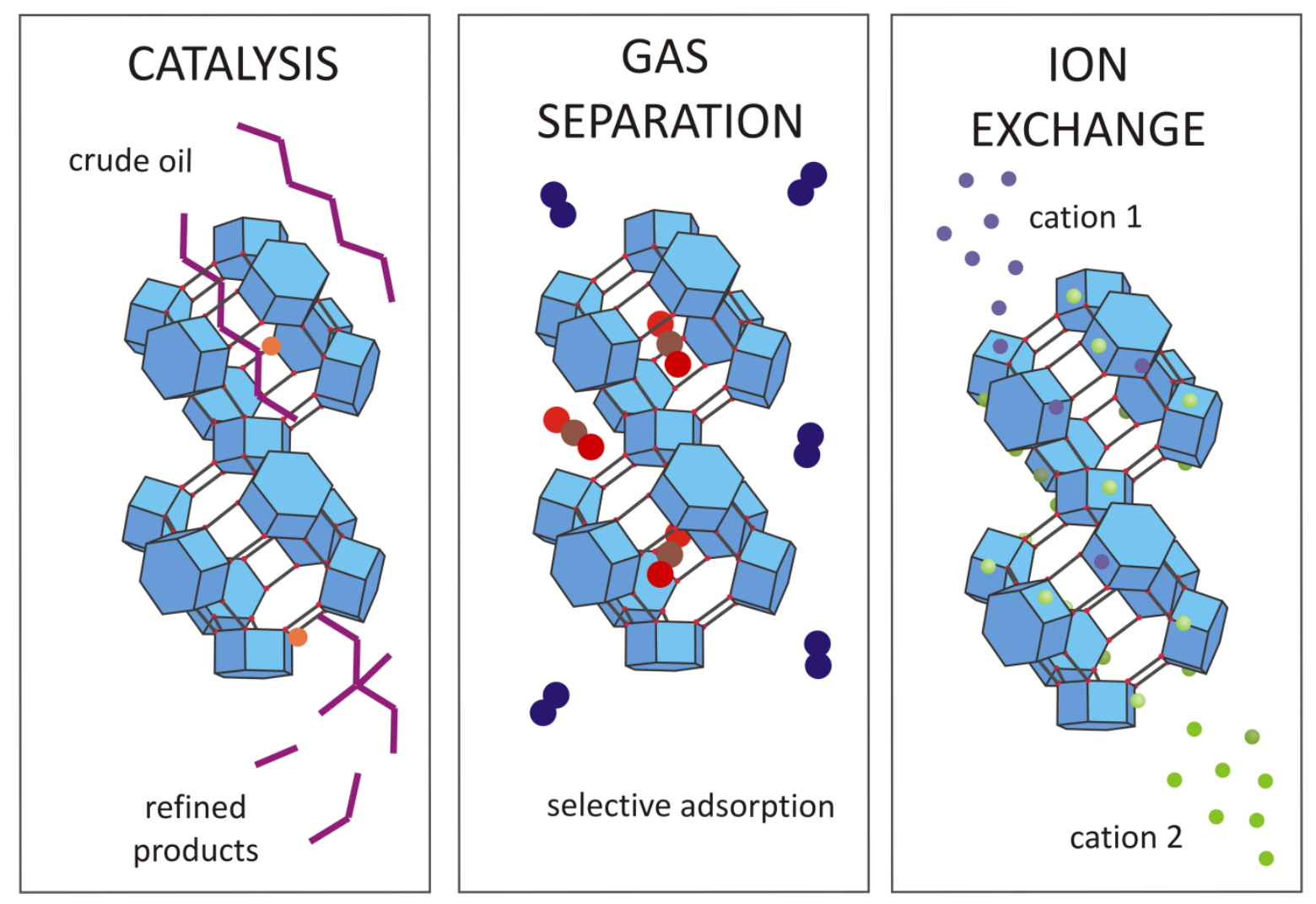

Figure 2.2.1 : Main applications of zeolites

The three main areas of application, illustrated in Figure 2.2.1, are as follows:

- Catalysis, which is probably the most significant economically and which embraces two main areas - acid and redox catalysis. The former is used on a huge scale in the petro-chemicals industry and exploits the acidity of zeolites due to both framework, Brønsted acid centers, as discussed above, but also, in some cases, extra-framework Lewis acid sites. ${ }^{24-27}$ The largest single application is in catalytic cracking of long chain hydrocarbons, (from the heavy fraction of crude oil) into shorter chain molecules appropriate for gasoline, where zeolite $Y$ is the catalyst and where the Brønsted acid center provides the active site. Other applications include isomerisation and hydrocarbon synthesis. Redox catalysis is effected generally by 
framework substituting metal ions of which TS-1 oxidation catalyst to above and discussed later is the classic example. In all cases, a key feature is the "shape selective" nature of the catalysis in which the microporous structure may control the product distribution by steric constraints on the reactants, products or transition state. ${ }^{20,28}$

- Gas separations, which may rely directly on the microporous architectures, as in molecular sieving, which essentially uses the pore structure to separate molecules of different size and shape. This type of separation technology should be distinguished from differential adsorption which may exploit the different affinities of molecules for centers (e.g. cations) within zeolites. ${ }^{8}$

- Ion Exchange which uses the hydrated zeolites materials whose loosely bound extraframework cations may be readily exchanged. ${ }^{24}$ These applications remain of substantial importance in water softening including detergency, while more specialist applications include removal of cations from contaminated water from mining or nuclear plants.

\section{Molecular simulations for unraveling zeolite synthesis}

Amongst the most fascinating questions in zeolite science are those concerning nucleation and growth of the materials in hydrothermal synthesis. This is a big area embracing many different topics and problems - nucleation, growth and agglomeration, surface processes (both growth and dissolution) and templating.

\subsection{Early stages of zeolite growth: Precursor nanoparticles - Basic Condensation Reactions - Early Aggregates}

This section concentrates on various modeling efforts that have been undertaken to understand the very first stages of zeolite synthesis, i.e. the initial stages of aggregation of small silicate and alumino-silicate clusters, where quantum mechanical calculations of the energetics of small silicate clusters have given useful information on some of the fundamental aspects of silicate and aluminosilicate oligomerisation. Modeling has proven extremely valuable in this field, as it is very difficult to extract information about individual reactions solely from experiment due to the simultaneous occurrence of many chemical events. Although the initial reactions are seemingly simple concerning basic silica condensation and polymerization reactions - accurate modeling of these steps has proven challenging. The origin must be traced back to the complexity of the reaction mixture, for which various factors such as $\mathrm{pH}$ of the solution, temperature, presence of structure 
directing agents all influence the outcome of the species formed. Given this intrinsic complexity, many theoretical techniques have necessarily been used. An overview of some of the computational methods used in this field is shown schematically in Figure 3.1.1. Quantum mechanical (QM) calculations are suited to investigate elementary reaction steps with high chemical accuracy, while more approximate methods based on interatomic potentials must be used when large numbers of molecules are needed in the simulations.

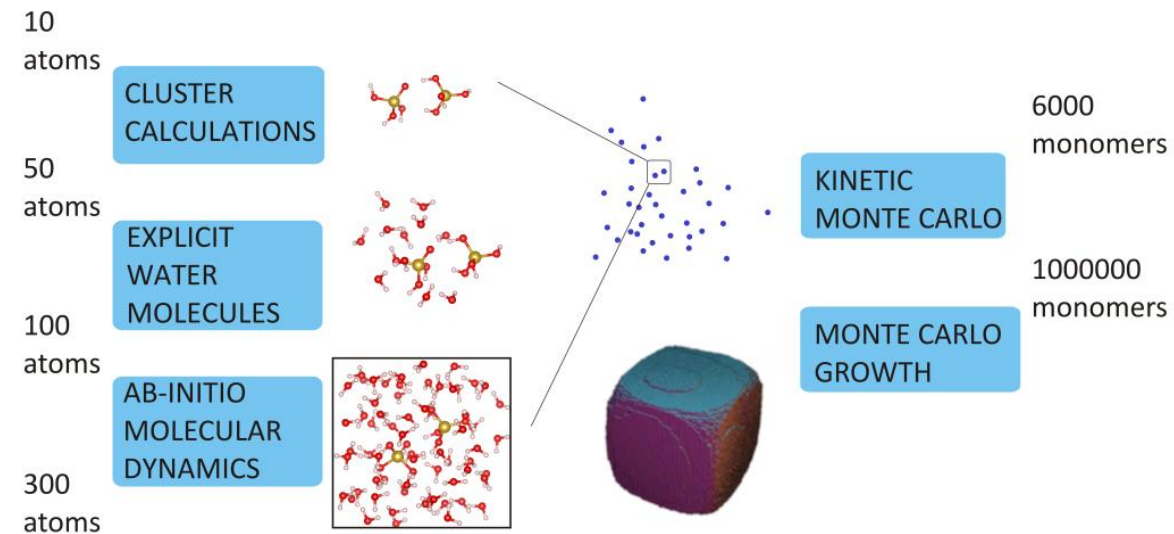

Figure 3.1.1 : Computational methods available in function of the system size.

Elegant NMR studies ${ }^{29,30}$ have clearly established the presence of small silicate clusters in synthesis gels; and studies of their structures and energies are clearly needed if we are to understand how they aggregate to form nuclei which subsequently grow into zeolite crystals. We now outline the approaches used in modelling these species in synthesis gels.

\section{Gas phase calculations}

Amongst the earliest calculations in this field was the work of Pereira et al. ${ }^{31-34}$, who studied the simplest possible silicon containing cluster, i.e. $\mathrm{Si}(\mathrm{OH})_{4}-$ orthosilicic acid - and its condensation to form a dimer - a reaction of fundamental importance as it involves the formation of an Si-O-Si bridge. Despite their relative simplicity, being confined to molecular mechanics and Density Functional Theory (DFT) calculations of species in the gas phase, useful results were obtained and the value of such studies was clearly established.

\section{Accounting for the water molecular environment using various solvent models}

After these initial gas phase studies, a series of papers appeared on the dimerization reaction concentrating on the anionic mechanism. Figure 3.1.2 shows the reaction profile of the anionic mechanism starting from $\mathrm{Si}(\mathrm{OH})_{4}$ and $\mathrm{Si}(\mathrm{OH})_{3} \mathrm{O}^{-}$. The anion attacks the neutral species to form an 
intermediate structure containing a 5-fold coordinated Si centre. Upon water removal, a negatively charged dimer is formed. All computational studies mentioned hereafter have used different models to mimic the water environment. The most straightforward approach is the usage of continuum models such as COSMO, PCM, CPCM,.... 35,36

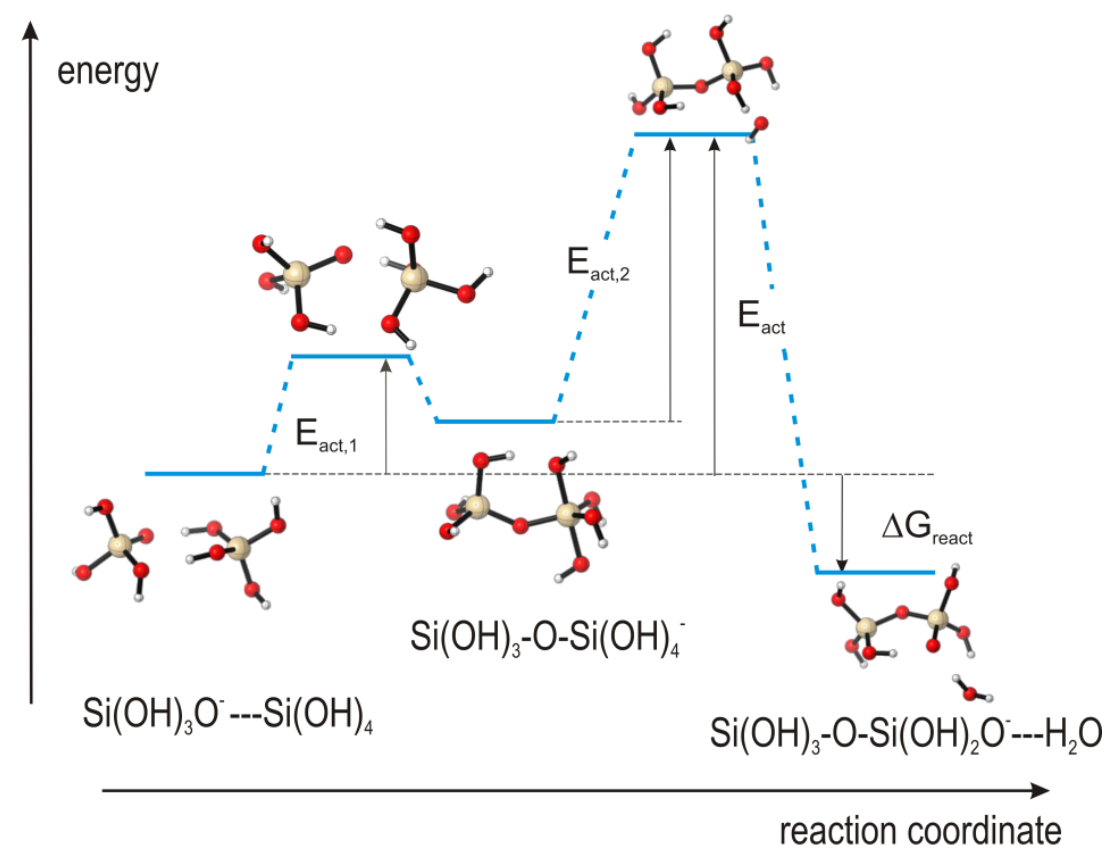

Figure 3.1.2 : Reaction profile for the silica dimerization reaction of $\mathrm{Si}(\mathrm{OH})_{4}$ and $\mathrm{Si}(\mathrm{OH})_{3} \mathrm{O}^{-}$through the anionic pathway.

Such an approach was employed by Mora-Fonz et al. ${ }^{37-40}$, where the basis was still DFT calculations, but explicit water was included and the whole silicate/water complex was embedded in the continuum-like representation of solvation available in the "COSMO" approach. ${ }^{35}$ They were able to calculate free energies as well as energies of reaction using standard statistical mechanical procedures for evaluating entropies of reaction. These calculations found good agreement with experimental data on the free energies of de-protonation and condensation of the $\mathrm{Si}(\mathrm{OH})_{4}$ clusters which encourages confidence in the approach.

Mora-Fonz et al. ${ }^{37-40}$ extended their studies to include the structures and energetics of small linear and ring oligomers. The clusters studied and the calculated free energies of condensation are shown in Figure 3.1.3 - 3.1.6. Analysis of the calculations gives valuable insight into some of the fundamental factors controlling the early stages of the oligomerisation reactions, in particular the calculations highlight the following: 
- The critical dependence of the condensation energies on the charge (i.e. degree of deprotonation) of the cluster. Favourable free energies of condensation require de-protonated clusters, which is in line with the use of high $\mathrm{pH}$ in zeolite synthesis

- The high stability of ring clusters, a factor which is almost certainly of substantial significance in driving the subsequent cluster growth.

- The key role of entropy in favouring the formation of ring clusters whose formation would not be predicted on the basis of the energy of condensation.

After these pioneering studies by Mora-Fonz on silica condensation reactions, a series of other relevant studies appeared using similar as well as more sophisticated computational procedures.

Table 3.1.1 lists the activation barriers of the two steps ( $E_{\text {act, } 1}$ and $\left.E_{a c t, 2}\right)$, the overall activation energy $\left(E_{\text {act }}\right)$ and the reaction energy $\left(\Delta G_{\text {react }}\right)$ for different methods: gas phase calculations and cluster calculations embedded in the continuum-like representation of solvation available in the "COSMO" approach. The values clearly show a distinct influence of the COSMO solvation on the barriers, demonstrating the importance of the implicit solvent model. The oligomers displayed in Figures 3.1.3-3.1.5 show multiple hydrogen bonds and it is to be expected that the addition of explicit water molecules could change the structure and energies of all these species significantly.

Water molecules can form hydrogen bridges with silica oligomers, but can also assist in the water removal step, effects that are not taken into account in implicit solvation schemes.

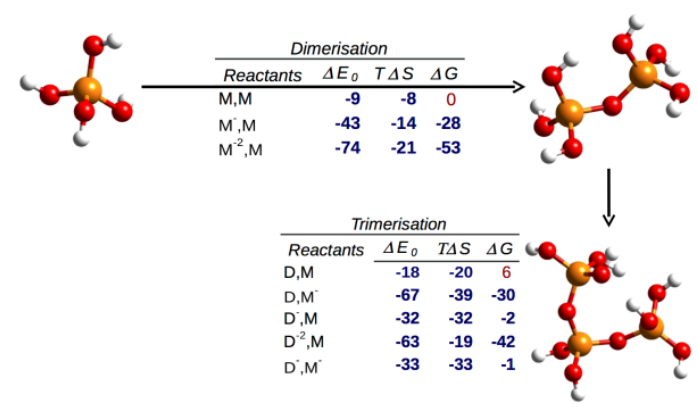

Figure 3.1.3 : Calculated reaction free energies $\left(\mathrm{kJ} \mathrm{mol}^{-1}\right)$ for the formation of dimers (D) from monomers $(\mathrm{M})$ and of trimers $(\mathrm{Tr})$ from dimer and monomer. Results are reported for different charge states of the clusters and for a temperature of $450 \mathrm{~K}$. Red indicates oxygen, orange silicon and white hydrogen atoms. Reprinted with permission from ref. ${ }^{41}$. 


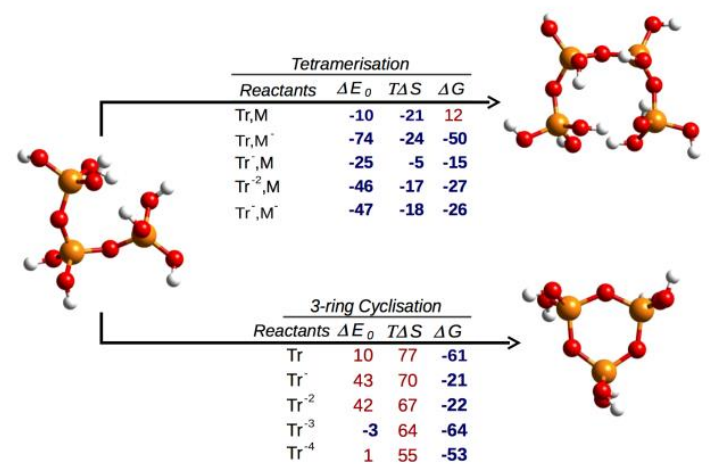

Fig. 3.1.4 Calculated reaction free energies $\left(\mathrm{kJ} \mathrm{mol}^{-1}\right)$ for the formation of tetramer and three-ring (via ring closure) for a temperature of $450 \mathrm{~K}$. Red indicates oxygen, orange silicon and white hydrogen atoms. Reprinted with permission from ref. ${ }^{41}$.

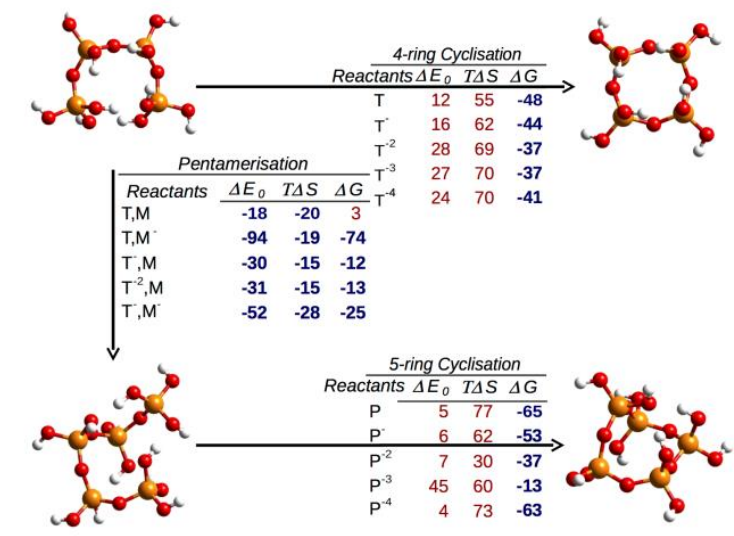

Fig. 3.1.5 : Calculated reaction free energies $\left(\mathrm{kJ} \mathrm{mol}^{-1}\right)$ for the formation of 4-ring, pentamer (P) and 5-ring from tetramer $(T)$ and monomer and via ring closure, for a temperature of $450 \mathrm{~K}$. Red indicates oxygen, orange silicon and white hydrogen atoms. Reprinted with permission from ref. ${ }^{41}$.

McIntosh ${ }^{42}$ tested the influence of explicit solvation by adding up to 12 water molecules to the dimerization reaction, while also including implicit solvation of the full cluster using a conductor-like polarizable continuum (CPCM) model. He initially argued that 8 water molecules suffice to converge the theoretical activation barriers and pre-exponential factors with respect to the number of water molecules. In a follow-up study describing the formation of trimers and tetramers, he lowered this number to 2 water molecules per silica tetrahedral. ${ }^{43,44}$ 
Table 3.1.1 : Activation energies and reaction energy in $\mathrm{kJ} \mathrm{mol}^{-1}$ in the silica dimerization reaction of $\mathrm{Si}(\mathrm{OH})_{4}$ and $\mathrm{Si}(\mathrm{OH})_{3} \mathrm{O}^{-}$through the anionic pathway.

\begin{tabular}{|c|c|c|c|c|c|c|c|}
\hline method & $\mathrm{T}$ & & $E_{\text {act1 }}$ & $E_{\text {act2 }}$ & $E_{\text {act }}$ & $\Delta G_{\text {react }}$ & \\
\hline in vacuo & $197 \mathrm{~K}$ & & 24 & 73 & 52 & -7 & ref. 45 \\
\hline COSMO & $297 \mathrm{~K}$ & & 28 & 101 & 90 & -8 & ref. 45 \\
\hline $8 \mathrm{H} 2 \mathrm{O}$ & $300 \mathrm{~K}$ & & 32 & 38 & 35 & -47 & ref. 43 \\
\hline CPMD & $300 \mathrm{~K}$ & & 44 & 42 & 61 & 25 & ref. ${ }^{46}$ \\
\hline CPMD & $300 \mathrm{~K}$ & $\mathrm{Li}^{+}$ & 69 & 36 & 94 & 36 & ref. 47 \\
\hline CPMD & $300 \mathrm{~K}$ & $\mathrm{Na}^{+}$ & 58 & 33 & 81 & 17 & ref. ${ }^{48}$ \\
\hline CPMD & $300 \mathrm{~K}$ & $\mathrm{NH}_{4}^{+}$ & 106 & 35 & 123 & 78 & ref. ${ }^{47}$ \\
\hline CPMD & $300 \mathrm{~K}$ & TPA $^{+}$ & 63 & 26 & 75 & 14 & ref. 49 \\
\hline
\end{tabular}

In addition, Mclntosh benchmarked a series of electronic levels of theory, selecting finally a hybrid approach as a good compromise between accuracy and computational cost. All hydrated clusters are first geometrically relaxed at the Hartree-Fock level of theory, a relatively inexpensive method. Then, the energy is obtained from a single-point energy calculation at the high MP2 level. ${ }^{42-44} E_{\text {act,2, }}$ the activation energy of the water removal reaction in the anionic mechanism (see Figure 3.1.2), is lowered considerably (from 101 to $38 \mathrm{~kJ} \mathrm{~mol}^{-1}$ ) when water molecules are included explicitly in the cluster calculations. The extra water molecules can assist the proton transfer that is occurring in this step. The overall reaction energy is more negative $\left(-47\right.$ versus $\left.-8 \mathrm{~kJ} \mathrm{~mol}^{-1}\right)$, because the released water molecule is readily accommodated in the hydrogen bond network of the solvent molecules. Explicit water molecules also have an influence on further oligomerization reactions. ${ }^{44}$ For instance, explicit water molecules do raise the barriers of the trimer cyclization reaction, but lower the relative stability of the silica three-ring. To understand the interplay between kinetics and thermodynamics, kinetic models are required. We will come back to this argument later. Trinh et al. ${ }^{50}$ found specific influence of intra- and intermolecular hydrogen bonding on the activation energies of subsequent oligomerization reactions (dimerization, trimerization, tetramerization) and ring closure reactions (3-ring, 4-ring). For instance, they attribute the high barriers for ring closure to the loss of intramolecular hydrogen bonds and the relatively low activation energies for the trimerization and tetramerization reaction to the favourable hydrogen bonds.

\section{Extension to alumino-silicate clusters}

The approach developed by Mora-Fonz and co-workers has also been extended to alumino-silicate clusters. ${ }^{51}$ In a series of studies, this work was able to identify a number of factors which are of particular relevance to zeolite synthesis, of which the most significant are as follows: 
- As would be expected and in line with our earlier discussion, there is a substantial qualitative difference between the results for "gas phase" (i.e. un-solvated) and those for solvated clusters. Only when solvation is included are the cluster stabilities in line with "Lowenstein's rule", which is observed in zeolites, and which requires the avoidance of Al-O-Al bridges.

- Following from the above, the calculations find high stability for the alumino-silicate anion dimer, [AIOSi(OH)6]-, which is predicted to a major player in zeolite growth.

- A mechanistic path was charted for the formation of the cubic double four-ring, $\mathrm{Si}_{4} \mathrm{Al}_{4} \mathrm{O}_{12}(\mathrm{OH})_{8}$, which is illustrated in Figure 3.1.6. This stable cluster is again predicted to be of central importance in the growth of zeolite structures.

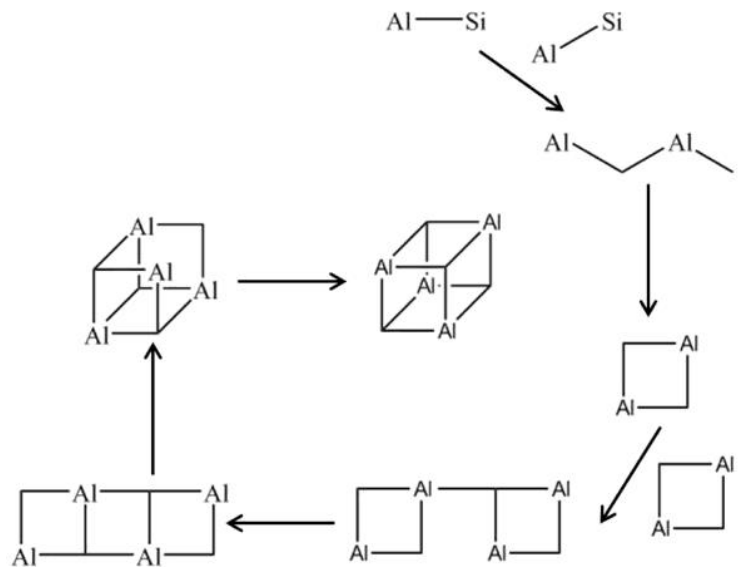

Figure 3.1.6 : A proposed nucleation mechanism for the formation of a D4R. Reprinted with permission from ref. ${ }^{51}$.

Clearly these calculations give useful insights into cluster stability and growth. Moreover, although much remains to be done, the calculations establish the viability and value of modelling in studying the early stages of zeolite synthesis. Further reviews of this work are given in references ${ }^{40}$ and ${ }^{41}$, which also place these studies into the context of the wider field of computational modelling of nano-clusters. ${ }^{52}$ The approach, however focuses on the thermodynamics of cluster oligomerisation and clearly we need more detailed information on kinetics which is provided by the methods which we now discuss.

\section{Accounting for the water molecular environment using ab initio molecular dynamics approaches}

To capture fully the influence of water, the oligomerization reactions were studied using periodically repeated water boxes and an ab initio molecular dynamics (MD) approach. $46,47,49,50,53$ For these calculations Car-Parrinello MD (CPMD) simulations were run, which are ideally suited to describe the dynamics of the water molecules. However, overall the dynamics of the system spans a wide range of characteristic time scales. The time step may not be chosen too large in order to sample the 
fastest motions of the system appropriately. Chemical transformations are rare events as their probability of occurring is very low, although once it occurs the event may be followed during a regular MD run of a few ps. To enhance sampling of interesting regions of the free energy surface a multitude of methods has been developed. ${ }^{54}$

Van Erp et al. proposed Transition Interface Sampling (TIS) simulations to study the oligomerization reactions. ${ }^{55} \mathrm{~A}$ method that has proven to be successful for the system under consideration is constrained dynamics, which effectively decouples the motion along a chosen reaction path from all other motions. By constraining a distance relevant to the reaction and performing CPMD simulations for a number of fixed distances along the path, the free energy can be obtained by thermodynamical integration of the average constraint force along the reaction path. ${ }^{46,50,56}$

For the dimerization reaction, the water removal reaction is no longer the rate limiting step. The water network around the newly formed dimer will assist the proton transfer. The barrier associated with the condensation of the two monomers is higher compared to the model with 8 explicit water molecules. This may be due to the fact that the water network needs to be rearranged before the $\mathrm{Si}$ O-Si bond can be formed. As opposed to all of the considered cluster calculations, the reaction energy is now positive ( 25 versus $-47 \mathrm{~kJ} \mathrm{~mol}^{-1}$ ). The water molecules in the solvent box have to accommodate the extra water molecule and this is thermodynamically not favourable. Water also has an important effect on the kinetics and thermodynamics of the formation of higher oligomers. For instance, the formation of silica 3-ring is favoured over higher branched and ring oligomers, which is not the case for cluster calculations, but corresponds better to experimental observations.

\section{Role of structure directing agents on initial oligomerization reactions}

The ab initio MD approach was also used to study the effect of counterions on the oligomerization reaction. When $\mathrm{Li}^{+}$or $\mathrm{NH}_{4}{ }^{+}$cations are present in the solution, the activation energies of the dimerization reaction increase compared to the case with no counterions $\left(E_{a c t}=94 \mathrm{~kJ} \mathrm{~mol}^{-1}\right.$ for $\mathrm{Li}^{+}$, $123 \mathrm{~kJ} \mathrm{~mol}^{-1}$ for $\mathrm{NH}_{4}{ }^{+}, 61 \mathrm{~kJ} \mathrm{~mol}^{-1}$ for no counterions). $\mathrm{NH}_{4}{ }^{+}$however has little effect on subsequent reactions, so the presence of $\mathrm{NH}_{4}{ }^{+}$increases the relative formation of larger moieties. The presence of $\mathrm{Li}^{+}$again increases the stability of linear and branched oligomers. ${ }^{47}$

Sodium cations also increase the overall barrier for the oligomerization reaction. The water removal reaction has a lower activation energy $\left(E_{\text {act, }}=33 \mathrm{~kJ} \mathrm{~mol}^{-1}\right)$ than for the reaction in absence of cations $\left(42 \mathrm{~kJ} \mathrm{~mol}^{-1}\right)$, which is the case for all cations. It should also be noted that there is no direct coordination between the $\mathrm{Na}^{+}$and the reaction center, the cation is present in the second solvation shell, so the influence of the cation may lie in the rearrangement of the water network. ${ }^{48}$ 
Tetrapropylammonium $\left(\operatorname{TPA}^{+}\right)$, a cation that is commercially used as a templating molecule, also has a distinct influence on the silica dimerization reaction. TPA ${ }^{+}$raises the barrier of the forward reaction by $14 \mathrm{~kJ} \mathrm{~mol}^{-1}$ and that of the backwards reaction by $25 \mathrm{~kJ} \mathrm{~mol}^{-1}$ with respect to a reaction where no counterion is present in the reaction. Moreover, the water molecules are more ordered around the reactants and products, whereas they are more mobile around the transition state. This behavior will certainly have an influence on the reaction profile and stresses the importance of using a dynamical approach with explicit water molecules. ${ }^{49}$ More details on the influence of structure directing agents on the influence on the complex assembly of highly ordered frameworks will be discussed in section 3.3.

\section{A multiscale approach to study initial oligomerization reactions}

It is computationally not feasible to study a realistic reaction mixture with QM methods, but kinetic models have been developed that use output of QM calculations to determine the reaction profiles of the relevant reactions and then describe the reacting mixture with thousands of monomers using Monte Carlo methods. Finally, nucleation and growth of zeolite crystals involve millions of silicon tetrahedral and for these very large systems, coarse-grained Monte Carlo techniques have been developed. ${ }^{57-60}$ Figure 3.1.1 illustrates the variety of available methods during the different stages of zeolite growth. When a complex reaction network is under consideration, it is difficult to determine the dominant species from kinetic and thermodynamic data alone. The reacting system can evolve in time; both forward and backward reactions may occur and there might be a delicate interplay between the height of the barrier and the stability of the species in the reaction, intermediate species can be converted into more stable products...

Kinetic Monte-Carlo (kMC) simulations facilitate the study of reactions in solution, i.e. a mixture of reactants, products and intermediates. The aim of $\mathrm{kMC}$ simulations is to follow the dynamical behavior of the solution and monitor the composition of the reaction mixture in time.

In this method, oligomers are considered as point-like particles, that can react with each other if they are close enough. The reaction rates can be determined up front, with cluster calculations or CPMD simulation, as described above. Since the effect of the solvent can be included in the DFT calculations, it is possible to make abstraction of the solvent in the $\mathrm{KMC}$ simulations. Finally, diffusion of the oligomers is treated analytically and is therefore also included in the reaction rates. Practically, after initialization of the system, neighboring oligomers can react (or not, depending on the barrier of the reaction) and the system is updated until the end of the simulation is reached. kMC elegantly followed the evolution of oligomeric species in solutions with varying $\mathrm{pH}$ and temperature. Near-neutral solutions favor the formation of linear species, while in more basic 
conditions, ring closure is favored. At pH 8, both linear growth and ring closure are optimal, resulting in the fastest oligomerization rates. As the solvent is not taken into account in the kMC simulations, the $\mathrm{pH}$ is factored into the rate constants. ${ }^{59}$

Another study discusses the structure-directing role of counterions for the oligomerization reactions.

58 The counterions that are considered are $\mathrm{Li}^{+}$and $\mathrm{NH}_{4}{ }^{+}$, their effect on the reactions is again included in the rate constants. The results of Zhang et al. from the kinetic Monte Carlo simulations are summarized in figure 3.1.7. $\mathrm{Li}^{+}$favors the formation of branched tetramers over 3-rings, whereas $\mathrm{NH}_{4}{ }^{+}$directs the formation of 3-rings.

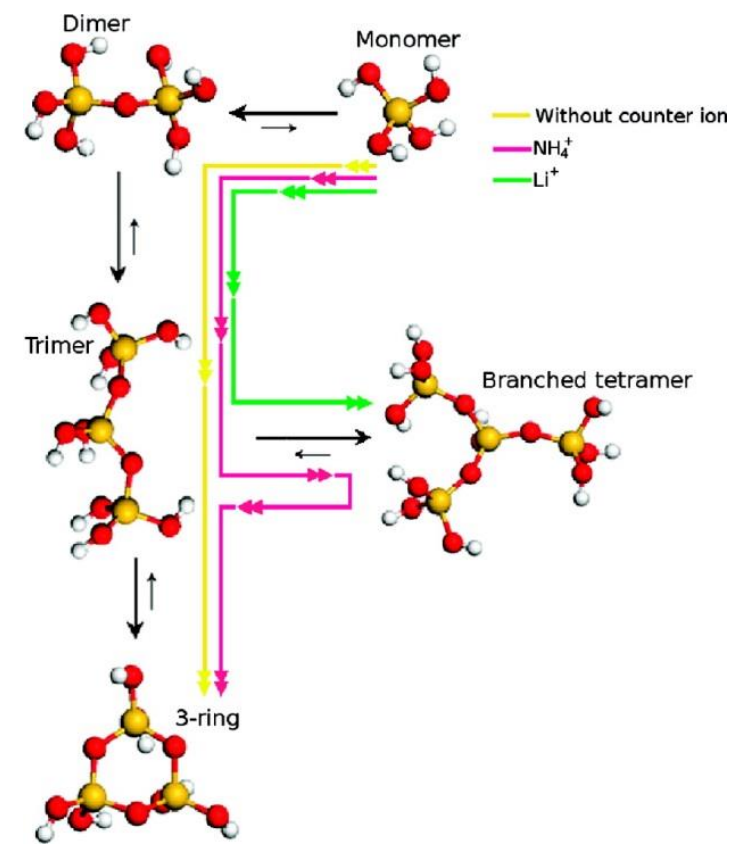

Figure 3.1.7 : schematic overview of structure-directing role of counterions. Reprinted with permission from ref. ${ }^{58}$.

These two examples show the ingenious interplay between various levels of modeling techniques.

\subsection{Surface Processes and Crystal Growth}

In this section we highlight the role of surface modelling in zeolite science and its impact in understanding crystal growth and dissolution mechanisms. We illustrate it with some examples offered by the work of Slater et al. ${ }^{61-64}$, where it is demonstrated how modelling can interact with high resolution TEM studies. We choose in particular their study of the surface structure and growth mechanisms of zeolite Beta C, by means of slab calculations employing DFT methods. Three stable surfaces were identified as illustrated in figs.3.2.1 a-c. Interestingly, the type 2 surface (figure 3.2.1 b) can be generated from the type 1 (figure 3.2.1 a) by addition of 4-rings and the type 3 from type 2, again by addition of a 4- ring to give the double 4-ring termination as shown in figure $3.2 .1 \mathrm{c}$. 


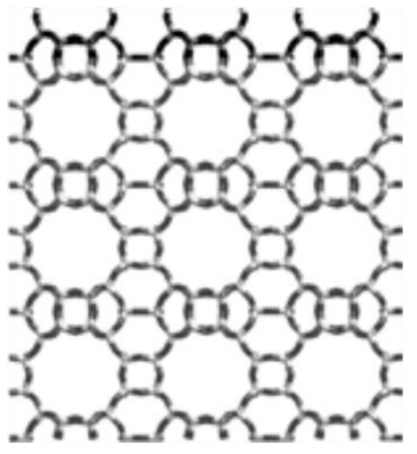

(a)

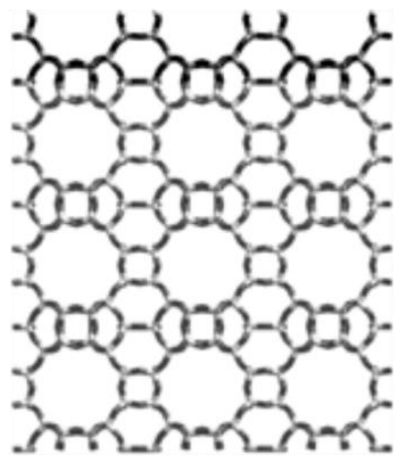

(b)

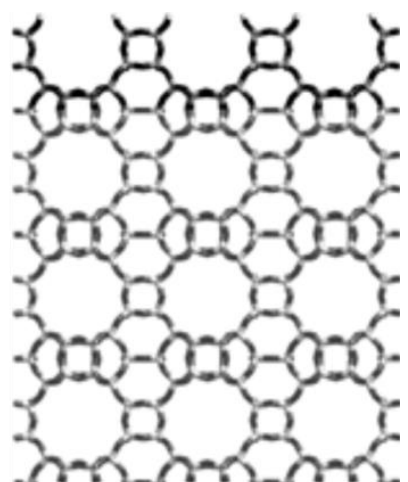

(c)

Figure 3.2.1 (a-c): illustrating the three stable surface configurations identified by DFT simulations: type 1 - upper; type 2 - middle; and type 3 - lower figure. All may be interconverted by addition of 4-rings. Reprinted with permission of ref. ${ }^{65}$.

Comparison with the HRTEM images obtained by Teresaki, Slater and co-workers ${ }^{65,} 66$ was particularly interesting. As will be seen in figure 3.2.2, terminations of type 1 and type 3 are observed experimentally and indeed there is a very nice agreement between calculated and experimental structures. Type 2 is not, however, observed experimentally which is at first sight puzzling.

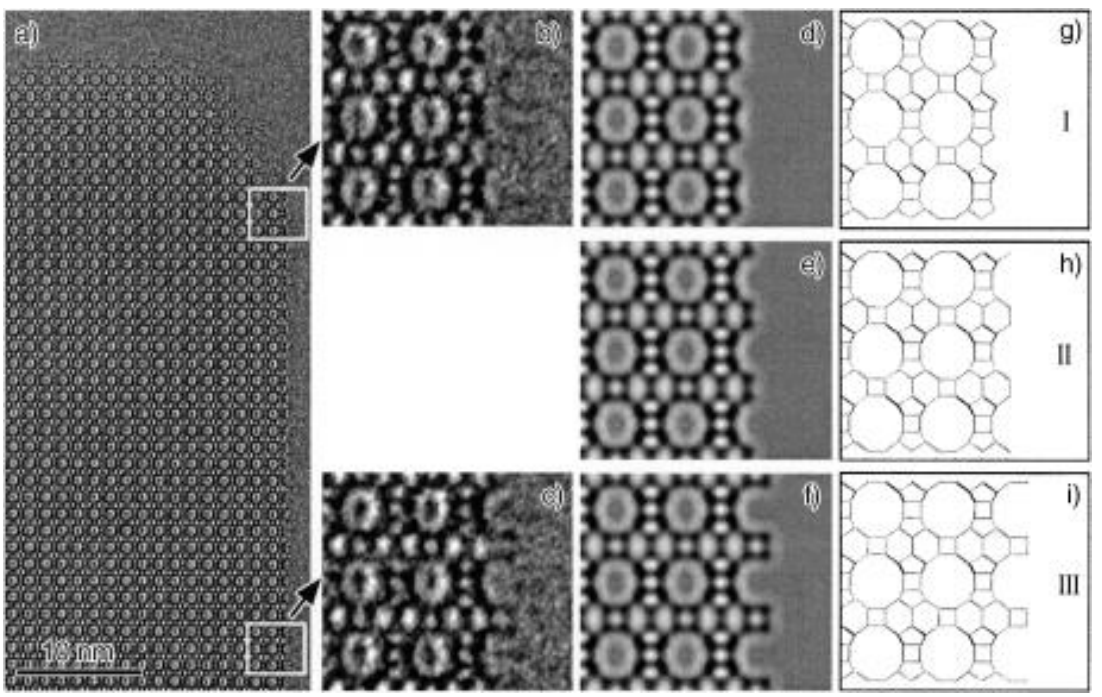

Figure 3.2.2 : a), b), c) Composite of HREM images. d), e), f) Simulated HREM images from relaxed surface structures g), h), and i), respectively, corresponding to a $90^{\circ}$ rotation of the type I, II, and III surfaces shown in Figure 3.2.1 a,b,c respectively. Reprinted with permission of ref. ${ }^{65}$ 
The key to understanding this conundrum was provided by molecular modelling. Energies associated to addition of 4-rings to the surfaces have been calculated. Addition of a 4-ring to the type 1 surface turns out to be an endothermic process. However, addition of a ring to the type 2, which converts it into a type 3 termination, is exothermic. This suggests that any type 2 formed will be rapidly converted during the growth process into type 3 and indeed that type 2 is a short lived intermediate in the whole crystal growth mechanism and hence is not experimentally observed. This study gave valuable insight into the mechanisms controlling crystal growth, as it reveals reliable models for the observed surface structures.

The DFT calculations described above have considered periodically repeated slabs of the crystal surface. The next step is to take a less idealized model of a zeolite surface that can exhibit different surface structures and can contain several types of surface defects. A full description of nucleation and growth with Monte Carlo techniques is computationally demanding and the problem should therefore at present be addressed by coarse-grained models. ${ }^{67}$ The experimental-theoretical studies mentioned before showed that surfaces terminated with closed-cage structures are significantly more stable than partially open structures. Although the actual crystal growth probably occurs via monomers or small oligomers, the stable cage structures will exist much longer at the surface. Therefore, the cages are the rate-determining entities and can be used to coarse-grain the problem, as the timescale of their existence can be decoupled from the much faster processes involving smaller units. ${ }^{63,65}$

Using this coarse-grained model, the zeolite structure is developed following thermodynamical rules. Boerrigter et. al. propose that the probability of growth and dissolution is given by

$$
\frac{P_{i 2 j}^{\text {growth }}}{P_{i 2 j}^{\text {etch }}}=\exp \left(\beta\left(\Delta U_{i 2 j}-\Delta U\right)+\beta \Delta \mu\right)
$$

The probability depends on the supersaturation $(\Delta \mu)$ and the energy difference between the surface site $\left(\Delta \mathrm{U}_{\mathrm{i} 2 \mathrm{j}}\right)$ and the bulk phase $(\Delta \mathrm{U})$. The energy of the surface sites can be estimated from the nearest neighbors (i2j). ${ }^{68}$ This method proved to be very successful in explaining experimental observations with Atomic Force Microscopy (AFM) and High Resolution Scanning Electron Microscopy (HRSEM).

In a study of the crystal growth of zeolite $L$, the parameters of the Monte Carlo simulations were tuned to obtain a good correspondence between the simulated and the observed crystal surface and aspect ratio of the surface. This allowed determining the stabilization energy of the cancrinite cages at $8 \mathrm{~kJ} \mathrm{~mol}^{-1}$ for each linkage to the growing crystal. ${ }^{61}$ 
For the crystallization of Zeolite A in absence of organic additives, Monte Carlo simulations show that the sharpness of the terrace corners is controlled by subtle changes in supersaturation. This was also observed with AFM and HRSEM images (Figure 3.2.3). ${ }^{69}$

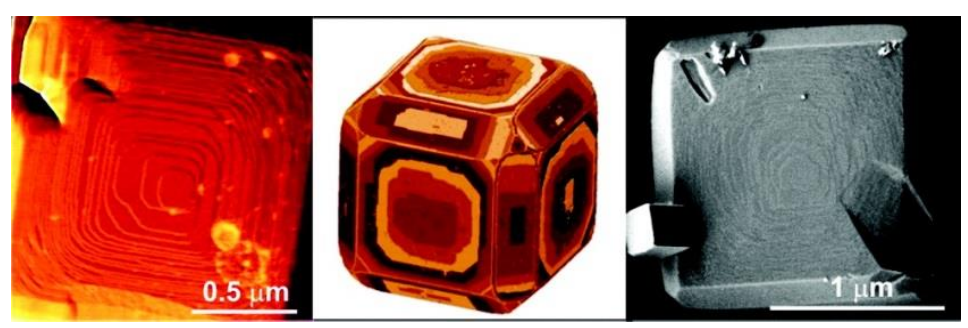

Figure 3.2.3: AFM image, Monte Carlo solution and HRSEM image for Zeolite A in medium supersaturation conditions. Reprinted with permission of ref. ${ }^{69}$.

When an SDA is included in the crystallization solution, it will change the relative stability of the cages that make up the crystal. Monte Carlo simulations showed that the final morphology of the crystal is dictated by small changes in the relative stability of the different units, i.e. the double fourring and $\alpha$ and $\beta$ cages for Zeolite $A .{ }^{70}$

\subsection{Modeling as a tool in template design}

The final topic, addressed in our overview on modeling applied to zeolite synthesis, concerns the use of organic templates, which is a well-established procedure in zeolite synthesis. Templates are used to guide synthesis towards specific microporous architectures and their selection often proceeds via trial and error perhaps inspired by intuition. Work in the 1990s showed, however, how modelling techniques could be used very effectively first in selecting and then in designing templates for targeted syntheses.

The first step ${ }^{71,72}$ was to use standard minimisation methods employing interatomic potentials to model template- host interactions. The binding energies were calculated for a range of alkylammonium and amine templates to zeolites hosts including several of the "ZSM" family $(5,11,18$ and 23). The calculations led to three main conclusions:

- The most effective template observed experimentally is generally that which has the highest calculated binding energy, provided both template - host and template - template interactions are taken into account.

- Following from the above, template - template interactions can have a substantial influence on template efficacy. 
- Modelling methods can predict accurately the sites occupied by template molecules within zeolite pores; where experimental diffraction data are available, the agreement between calculations and experiment is good.

This work clearly allowed screening of templates for a particular synthesis: a range of candidates can be examined using these inexpensive calculations and the optimal candidate selected. The next step was to move towards design. Here, Lewis and co-workers ${ }^{73}$ took approaches developed in the field of drug design and adapted and implemented them in the design of zeolite synthesis templates. In drug design, the aim is to identify a molecule with the optimum fit to a receptor site; in template design, the molecule must optimise the interaction with the target host lattice. The drug design field had developed procedures to "grow" molecules inside the target receptor; the Zebeddee code developed by Lewis et al. grows templates inside the target host. The procedure starts with a "seed" molecule to which functional groups from a fragment library are added; a range of operations may then follow, including bond rotation, translation and energy minimisation as illustrated diagrammatically in Figure 3.3.1. The procedure continues until the optimum template is generated, although commonly, several possible templates may be identified. The figure illustrates the first successful application of the code: a di-substituted cyclohexane derivative was designed and successfully used in the synthesis of a levyne structured alumino-phosphate.

Perhaps the most successful application of the approach ${ }^{74}$ was the design of the template for the synthesis of a chabazitic structured alumino-phosphate (denoted "DAF-5"). Application of Zebeddee predicted the 4-piperidinopiperidine structure, which fits nicely inside the pores of the target host as illustrated in Figure 3.3.2.

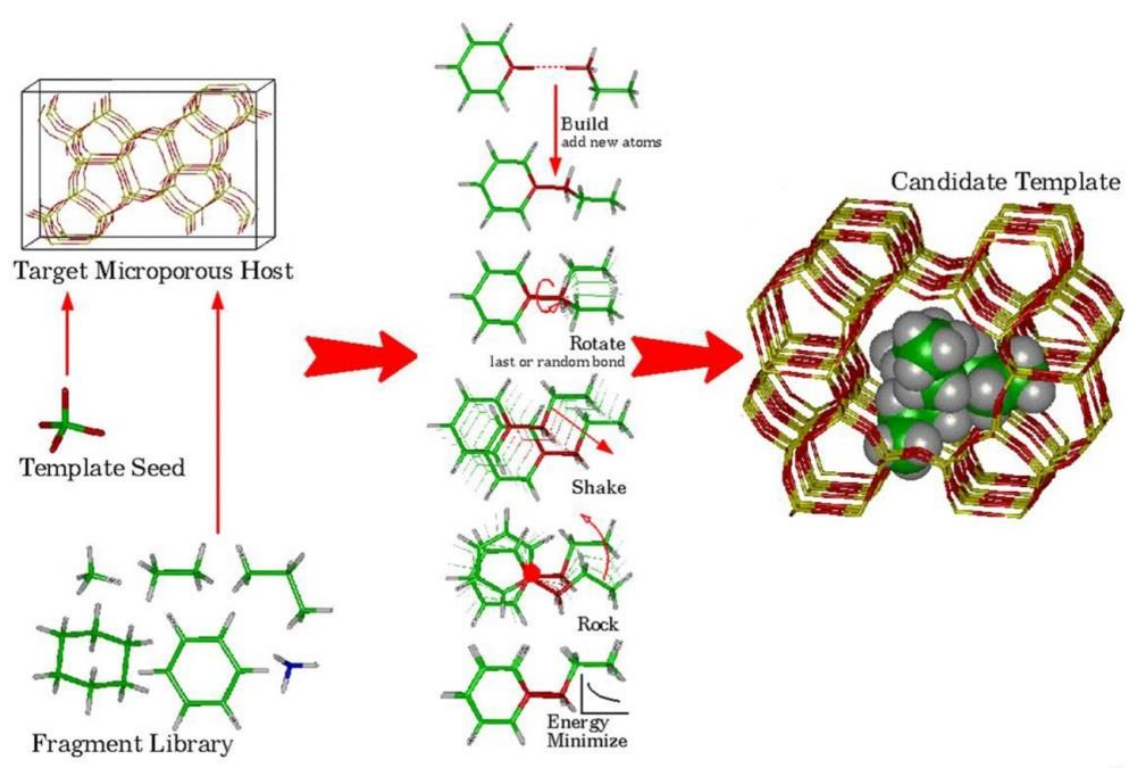


Figure 3.3.1 : Diagrammatic illustration of template design procedure in the Zebeddee code. A real example is shown in which the code was used to design a di-substituted cyclo-hexane derivative to synthesise a levyne structured alumino-phosphate. Reprinted with permission of ref. ${ }^{74}$.

Subsequently, the structure predicted by the calculations was verified by the application of synchrotron based, micro-crystalline diffraction methods which allow single crystal experiments to be performed on the larger crystallites extracted from a powder sample. ${ }^{75}$ This work once again illustrates the close integration of modelling with experiment in zeolite science.

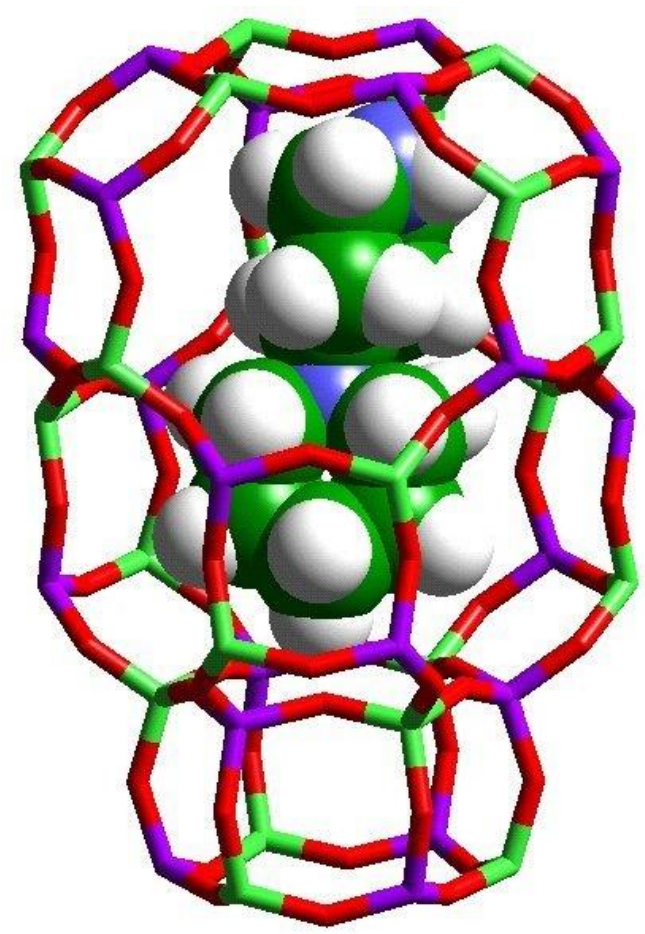

Figure 3.3.2 : Predicted structure of 4 piperidinopiperidine inside the pores of DAF-5. ${ }^{74}$

The value of modelling in understanding templating and structure direction has been illustrated in several recent studies, especially the wide-ranging investigations of Cora, Gomez-Hortiguela et al.. ${ }^{76-}$ ${ }^{78}$ Their work has included investigation of chiral supra-molecular assemblies of template molecules 79 , which may be used to introduce chirality into the target material; and they have extensively investigated fluorinated templates ${ }^{80,81}$ in which the fluorine can play a number of roles, of which its ability to interact with other ions leading to co-operative effects is particularly significant.

Some of the observations made by Lewis et al. ${ }^{74}$ were later confirmed by Verstraelen et al. ${ }^{82}$ in a multi-level modeling study on the silica-template interactions during the initial stages of zeolite synthesis. Both static ab initio and classical MD studies were performed to obtain insight into the interactions between the tetrapropylammonium ions (TPA+) and Silicalite-1 precursors. As soon as 
the typical 10 ring-channels of ZSM- 5 are formed from the smaller oligomers, the TPA+ template is partly squeezed out of the cavities from the precursor units, as is schematically shown in figure 3.3.3. It is clear that the "half in -half out" structures are more stable than the structure where the TPA is fully located inside the precursor. Optimization of the structures without the template molecules resulted in global minima structures where the channel of the initially formed silica precursor collapsed in accordance with the observation of Lewis et al.. ${ }^{74}$ They found that a template prevents the decrease in surface area of the open-structured fragment. The influence of solvent was tested using the COSMO methodology, which was also successfully applied by Mora-Fonz et al.. ${ }^{37}$ In all cases a substantial stabilization is found, which is larger for ethanol than for water. These observations are in agreement with the experimental results of Kirschhock et al. who found that precursor species were longer lived in ethanol compared to water. ${ }^{83,84}$

The TPA-silica structures in which the TPA is positioned partially out of the nanobricks, were further investigated on their ability to eventually form large aggregates. Initially this was done from a purely geometrical point of view using a dedicated software program ZEOBUILDER ${ }^{85}$ that allows the construction of complex molecular structures starting from building blocks. Using only geometrical considerations and a condensation algorithm, searching for the optimal connections between sets of oxygen pairs and MFI-type nano-crystals, could be constructed containing 12 aggregated $\mathrm{Si}_{33}$ precursors as found from the static $a b$ initio calculations (structures $A$ and $C$ in figure 3.3.3). The resulting small nano-crystallite in which the template resides close to the channel intersections is shown in Figure 3.3.4. 

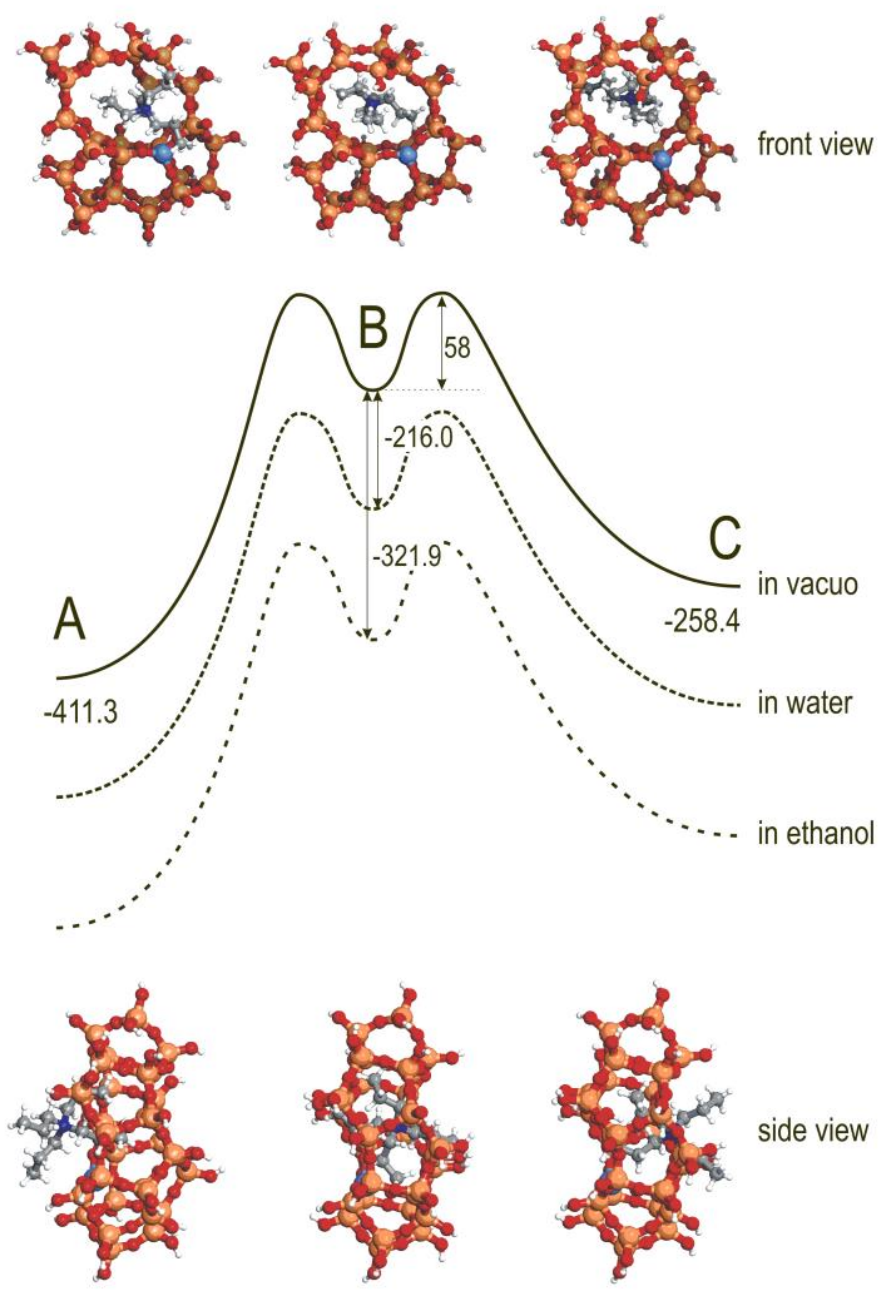

Figure 3.3.3 : Relative energies in $\mathrm{kJ} \mathrm{mol}^{-1}$ between three stable structures ( $\mathrm{Si}_{32} \mathrm{Al}^{-}$precursor and $\mathrm{TPA}^{+}$) at the GPW/PBE/TZ2VP-PSP level. Reprinted with permission from ref. ${ }^{82}$.

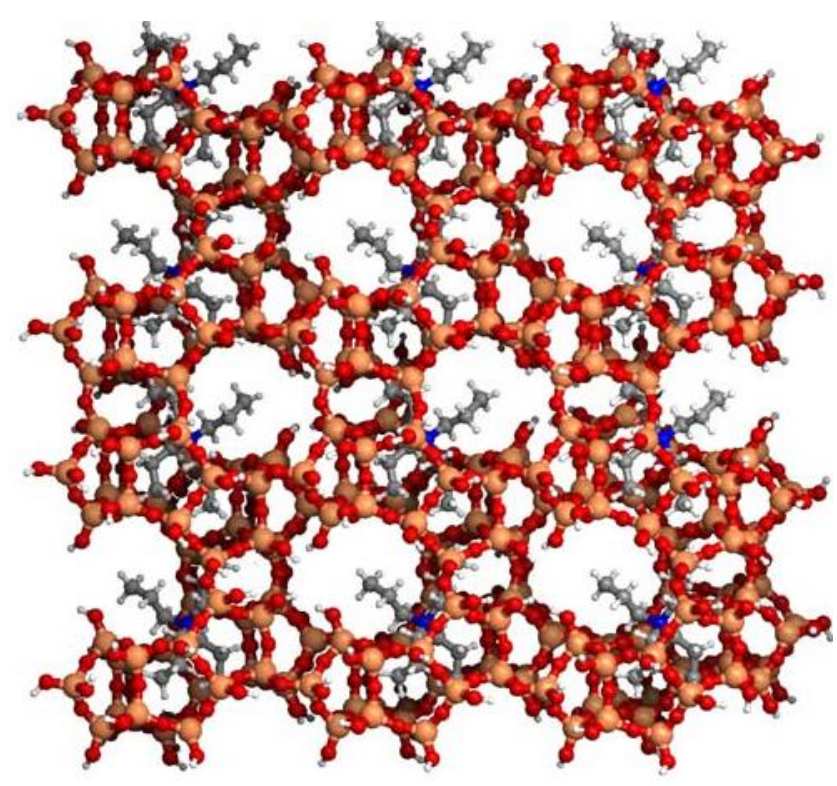


Figure 3.3.4 : MFI-type nano-crystal containing 12 aggregated $\mathrm{Si}_{33}$ precursors constructed from stable TPA-complexes using the ZEOBUILDER program. ${ }^{85}$ Reprinted with permission from ref. ${ }^{82}$.

The conclusions from the static calculations were later confirmed by a classical MD simulation, which accounted for the full molecular environment. More recent studies on the template framework interactions have recently appeared. ${ }^{86,87}$ Overall, this and other work illustrates the detail and complexity of template - template and template- host interactions which can now be elucidated from modelling methods.

Finally we note that a complementary approach to template selection has been proposed recently by Pophale et al. ${ }^{88}$, who use a data base comprising a library of available reagents. The compounds are then evaluated on the basis of their stability during synthesis and other factors which include energy of interaction with the target zeolite. The method has the added advantage of including the likely availability of a proposed template as a criterion for selection.

\section{Structural Modeling}

Some of the earliest successes of zeolite modelling relates to modelling of crystal structures. In this section we illustrate their role in modelling and predicting new zeolite structures, and addressing the conversion of existing zeolites. We also briefly discuss the role of modelling techniques in investigating the external surfaces of zeolites.

\subsection{Hypothetical Zeolites and Structural Modelling}

As we have stated, a key goal of computational chemistry is to be able to design a zeolite, or zeolitebased material, for a specific application. Enormous progress has been made in understanding many aspects of zeolite synthesis and chemistry, though at the present time we are still a long way from being able to produce a pre-defined, previously unsynthesized zeolite structure from scratch. Nevertheless there is great value in exploring the possible zeolite topologies, firstly to try to understand in general terms why such a small proportion of them have so far been synthesized, and secondly to model their likely properties, producing a "library" or database of potential zeolite framework structures. Such a database can be readily screened in order to identify hypothetical zeolites with particular properties, for instance pore volume, internal surface areas, or accessibility to molecules of certain dimensions. 


\section{Enumeration of hypothetical zeolite topologies.}

The characteristic feature of a zeolitic framework structure (in addition to porosity) is that it can be considered as a network of corner-sharing tetrahedra, normally of composition $\mathrm{TO}_{4}$, (where $\mathrm{T}$ may be $\mathrm{Si}, \mathrm{Al}, \mathrm{P}$, etc.). The positions of the central T-atoms thus form the vertices of a 4-connected net which uniquely defines each zeolite topology. Zeolites are of course also periodic structures, and contain well-known structural building units, meaning that it is relatively facile to predict new topologies using a model-building type of approach where known building units are linked together in different arrangements.

Systematic enumeration of hypothetical zeolites using computational methods commenced in the 1990s when Treacy and co-workers ${ }^{89}$ were able to generate a series of structures using the symmetry constrained intersite bonding search method (SCIBS), a combinatorial method in which all possible zeolitic structures are identified within a certain space group and for a given number of unique nodes (T-atoms). Deem and co-workers ${ }^{88,90,91}$ subsequently used the simulated annealing program ZEFSA II, originally intended for zeolite structure solution, to generate and refine over 2.6 million zeolite frameworks (the total possible number of 3-periodic 4-connected nets is in fact infinite). These methods and others are described in greater detail in the comprehensive review of $\mathrm{Li}$ and $\mathrm{Yu} .{ }^{92}$ An alternative approach involves the use of tiling theory, which exploits the fact that zeolites can be represented as space-filling tilings of polyhedra (see Fig. 4.1.1). Delgado Friedrichs et al. ${ }^{93}$ showed that all possible tilings of space may be generated for a given number of unique tetrahedral nodes, i.e. a node is where four tile edges meet at each vertex. This suggested the possibility, albeit within defined boundaries, of a "complete" solution to generating hypothetical zeolite topologies. The generated graphs were converted into zeolite structures by appropriate positioning of silicon and oxygen atoms and optimised using a forcefield method. ${ }^{94-98}$ The several hundred zeolite types thus enumerated contained a number of energetically feasible structures, as well as possessing many interesting structural features.

On a side note, it is worth mentioning that the majority of work that has hitherto appeared on hypothetical zeolites has assumed (largely for convenience) that they adopt purely siliceous frameworks. However modelling aluminum distributions in zeolites is a parallel activity which clearly has great impact when it comes to predicting properties of the materials, such adsorption capacities. An example is the procedure of Garcia-Perez et al. ${ }^{99}$ for identifying the likely aluminium and extraframework cations in known zeolites, based on the best match between matching experimental adsorption data with force field simulations. This procedure was further used in an extensive study predicting $\mathrm{CO}_{2}$ adsorption in hypothetical zeolites with varying Si/Al ratios. ${ }^{100}$ 


\section{Feasibility}

Having enumerated large databases of millions of potential zeolites, assessing the feasibility of such frameworks has proved a more persistent and challenging issue. Initial studies focussed on the thermodynamics of formation of the zeolite, usually in a pure silica composition. This can be evaluated using a cost function, with the structures commonly being energy-minimised in a program such as GULP ${ }^{101}$ using a classical force field. If the lattice enthalpy of an optimised framework with respect to that of quartz is too large, it is assumed, by analogy with the known zeolites, that it cannot be synthesized. Foster at al. ${ }^{96}$ further introduced a feasibility factor based on the energy and density of the material, taking into account the variation of lattice energy with density among synthesized materials.

More recently it has become apparent that a measure based principally on lattice energy is not in itself a sufficiently discriminating criterion. There are a great many hypothetical frameworks which have apparently feasible framework energies - for instance within $30 \mathrm{~kJ} \mathrm{~mol}^{-1}$ of quartz by the widely-used SLC force field. ${ }^{102}$ However many of these possess structural features not commonly found among the known synthesised zeolites, i.e. the 225 structures found in the Database of Zeolite Structures. Zwijnenburg et al. ${ }^{103}$ correlated the calculated lattice energy of the materials with their degree of distortion of their $\mathrm{TO}_{4}$ tetrahedra in the ideal, optimized geometries. The preference of synthesized zeolites, particularly those known in a high-silica composition, to have highly regular tetrahedral geometries is not obvious from energy calculation alone, since slight distortions in bonding geometry affect the energy in a very subtle fashion. This focus on local geometry was further explored by Li et al. ${ }^{104}$ who proposed the Local Interatomic Distances (LIDs) criteria, based on observed correlations between intratetrahedral geometry ( $\mathrm{T}-\mathrm{O}$ and $\mathrm{O}-\mathrm{O}$ distances), as well as T-T distances. These conditions serve further to narrow down the set of hypothetical frameworks which might be feasible synthetic targets. For instance, out of a sample of 665 structures, 372 fell within the feasible energy range, but of these only 181 fulfilled all the LID criteria. 

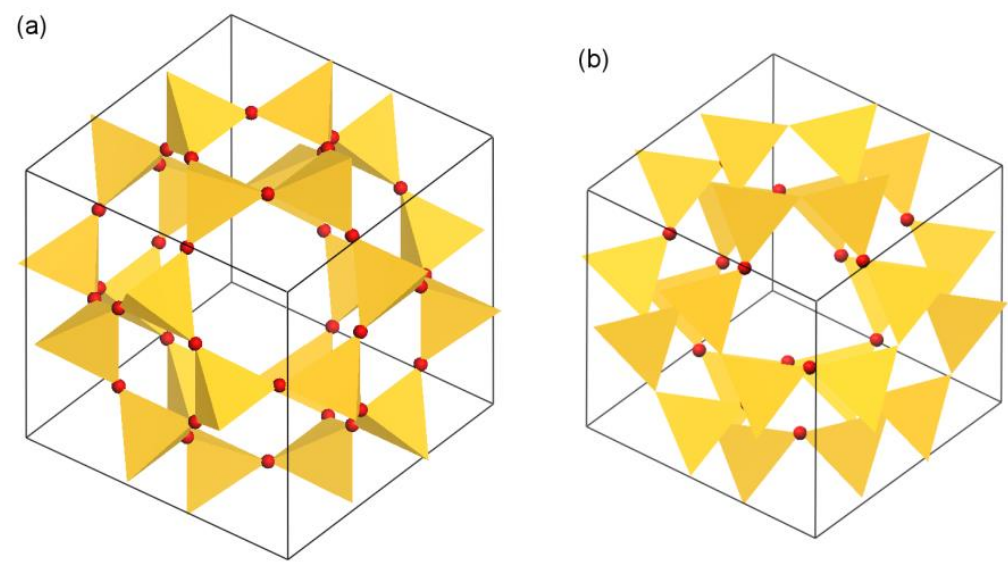

Figure 4.1.1 : Representation of the SOD structure type at (a) minimum and (b )maximum density in the flexibility window, assuming a cubic cell (adapted from Kapko et al. ${ }^{105}$ ).

Flexibility is another key property of zeolite type structures. Due to the geometric inflexibility of the $\mathrm{TO}_{4}$ (or at least $\mathrm{SiO}_{4}$ ) tetrahedra, the dynamics of zeolite frameworks are traditionally described in terms of Rigid Unit Modes (RUM) in which the tetrahedra are considered completely rigid. The shared oxygen atoms act as hinges, and the variation of the T-O-T angles characterises the framework flexibility. Using a purely geometric Rigid Unit model, Sartbaeva et al. ${ }^{106}$ discovered that many synthesized zeolite frameworks display a "flexibility window", a range of density over which the structure can exist whilst retaining all bonding connectivity and without distorting any of the coordination tetrahedra (see fig. 3.3.7) Kapko et al. ${ }^{105}$, extending the study, found that the flexibility window is exhibited by virtually all of the synthesized zeolites. Generally the range of the flexibility window is significant, ranging between 10 and $20 \%$ of the ideal geometry for many zeolites, and considerably more in some cases. In these simulations energy is not included and in fact substantial energy would be required to access most of the configurations in the flexibility window. Nevertheless it is surmised that having a large configurational space is important in zeolite formation, where vibrational entropy is a key factor. Alternatively, the flexibility may come into play at the nucleation stage, where it is important in enabling template molecules to be accommodated. Further studies on a sample of hypothetical zeolites ${ }^{107}$ found that only $10 \%$ of these showed flexibility, and moreover that the flexible frameworks tended to share certain properties in terms of internal surface area, pore volume and accessibility. It seems likely therefore that this type of flexibility, which is inherent to the network topology of the zeolite type framework, is a fundamental property of zeolite structures and that structures which lack it are not feasible synthetic targets. It should also be noted here that, while most simulations have been carried out assuming a siliceous 
composition, there are several known synthesized zeolites which are only flexible when tetrahedra of different sizes are considered (e.g. $\mathrm{SiO}_{4}$ and $\mathrm{AlO}_{4}$ ). Returning to the concept of tiling, recent work of Blatov and co-workers ${ }^{108}$ has also shed light on the area of zeolite feasibility. As mentioned briefly already, zeolite structures can be described in terms of three-dimensional tilings of tetrahedra. A unique tiling may be generated for any topology, known as the natural tiling (see Figure 4.1.2). ${ }^{109}$ The natural tilings of all 225 known zeolites are shown in the Database of Zeolite Structures. ${ }^{110}$ The tiles represent a huge range of polyhedra, from large cage-like structures to small building units such as the cube and hexagonal prism. Blatov et al. ${ }^{108}$ have further analysed the tilings and proposed an alternative model based on Natural Building Units (NBU) (see Figure 4.1.3). These form a much more restricted group of mostly small cages and units, and the majority (163 out of 201) of synthesized zeolite structures can be decomposed into a combination of NBUs and small rings. As in the case of the zeolite secondary building units ${ }^{110}$, but distinct from the natural tilings, the NBUs do not share Tatoms, and so are conceptually "building units".

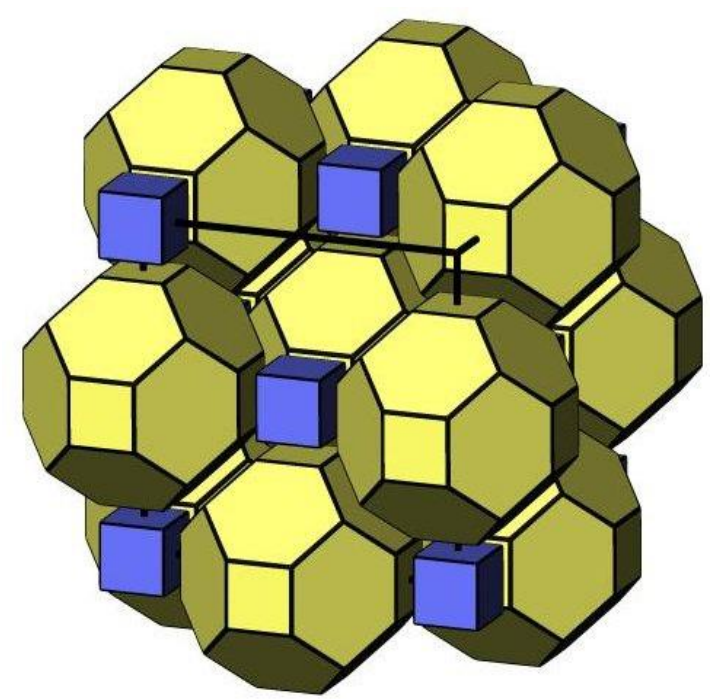

Figure 4.1.2 : Natural tiling from zeolite type AST. Figure from ref. ${ }^{19}$.

The packing of NBUs is potentially a very powerful concept as it may provide a direct link between the zeolite topology and synthetic mechanism. An attempt to decompose a set of hypothetical zeolites into NBUs succeeded with a much smaller proportion (237 out of 1220), potentially providing another feasibility criterion.

To conclude this section, it can be stated the field of structural and topological characterization of zeolites is a highly active one with several different strands of investigation ongoing, including framework flexibility, tilings, and characterization in terms of local geometries (bond lengths and angles) and of pore dimensions and accessibility. In trying to predict feasible zeolite structure types, 
a number of important concepts have been established. Framework flexibility is a bulk property of a zeolite structure, but is clearly also linked to the regularity of coordination tetrahedra and hence to local bonding. The NBU model leads us to think more about aspects of medium-range structure which are otherwise not conveniently described. The next few years are sure to see these ideas further developed and for our knowledge of zeolite topology and its relationship to synthetic pathways and feasibility to be further extended.

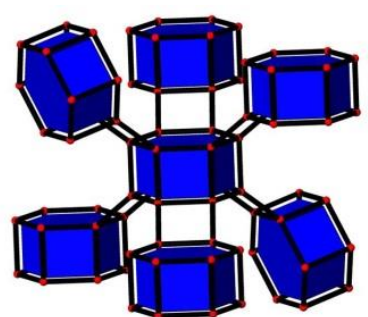

(a) AEI

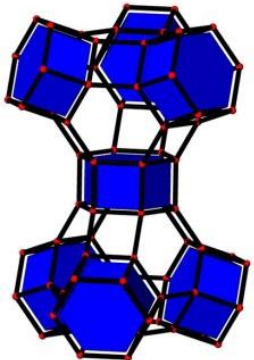

(d) EMT, FAU

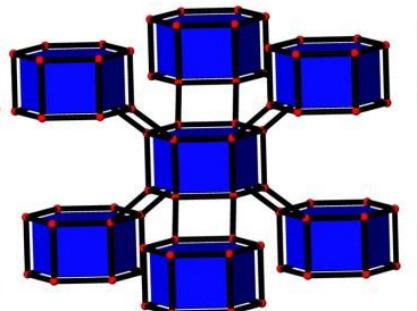

(b) AFT, AFX, CHA

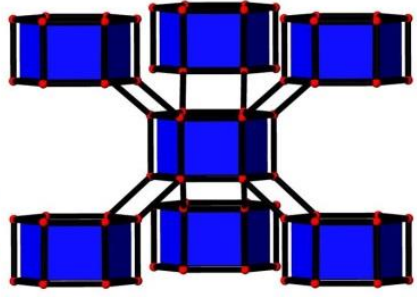

(c) AFX, GME

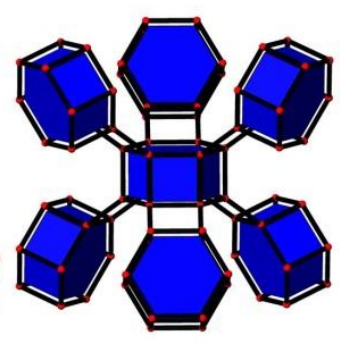

(e) KFI

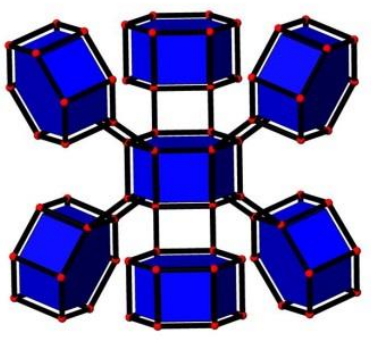

(f) SAV

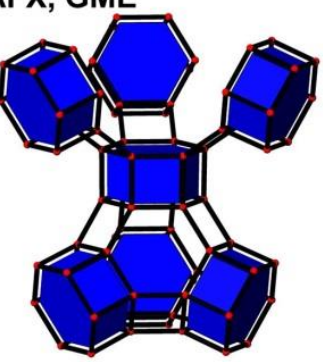

(g) TSC

Figure 4.1.3 : Packings of NBUs (double 6-rings) for 11 Zeolite Structure types (from ref. ${ }^{108}$ )

\subsection{Conversion of existing zeolites}

A promising direction in the rational design of new zeolites is to convert existing zeolite frameworks into new structures. Layered zeolites are promising candidates for such transformations, because it is in principle possible to keep the layer anatomy intact while reshaping the interlayer structure. A showcase example is the conversion of the germanosilicate IM-12 framework (UTL topology) into the all-silica COK-14 structure (OKO topology). ${ }^{111}$ The germanium four-rings in the IM-12 framework are highly reactive and render the framework unstable for industrial applications at high temperatures and in harsh environments. The same reactivity and instability can however be exploited to dislodge the germanium and create a contracted all-silica framework with pores that are slightly smaller, but much more stable. Several computational studies have helped in understanding the mechanisms that are at play.

Two mechanisms are proposed in literature. Verheyen et al. suggest acid leaching whereby the germanium four-rings are dislodged from the parent IM-12 structure and initially accommodated in 
the pores (Ge-COK-14). Further leaching removes all germanium and achieves contraction of the framework (Figure 4.2.1). The as-synthesized structure is a systematically interrupted framework (-COK-14), that can be reversibly interconverted to the fully connected analogue (COK-14) through annealing at high temperature. ${ }^{111,112}$ The leading dash in -COK-14 denotes the framework interruption.

Roth et al. synthesized the same material with the multistep ADOR strategy (Assembly-DisassemblyOrganization-Reassembly). The method first disassembles the assembled UTL zeolite into layers, then organizes the layers using a structure directing agent and subsequently reassembles the layers into a new framework topology (see Figure 4.2.2). Two possible final topologies are reported, one that consists of the two layers directly connected and one with a silicon four-ring in between the layers (corresponding to OKO). ${ }^{111,113,114}$

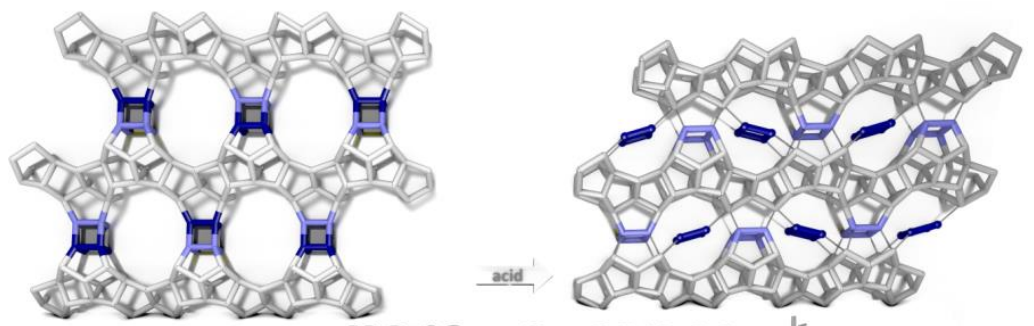

IM-12 Ge-COK-14 㞔

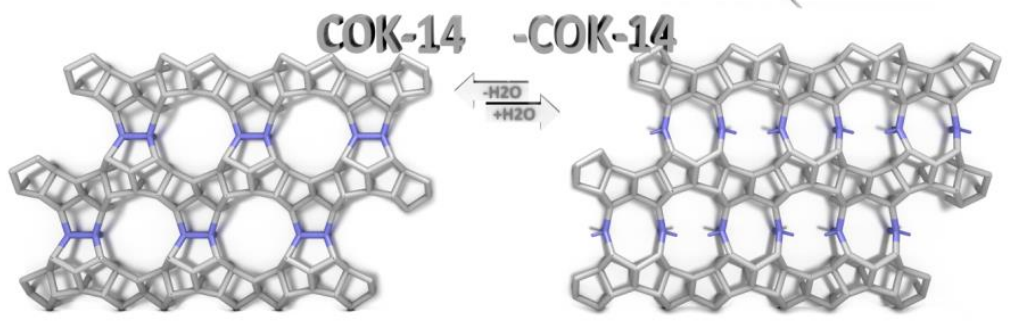

Figure 4.2.1 : An inverse sigma transformation applied on an IM-12 zeolite leading to an interrupted -framework (-COK-14), that can be annealed on calcination $\left(550^{\circ} \mathrm{C}\right)$ to form a closed frame COK-14. Si-atoms in layers are presented in grey, while Si-atoms in layer-connecting Si-4Rs are given in light blue. ). [reprinted with permission from ref. ${ }^{111}$. Copyright 2012 Macmillan Publishers]. 


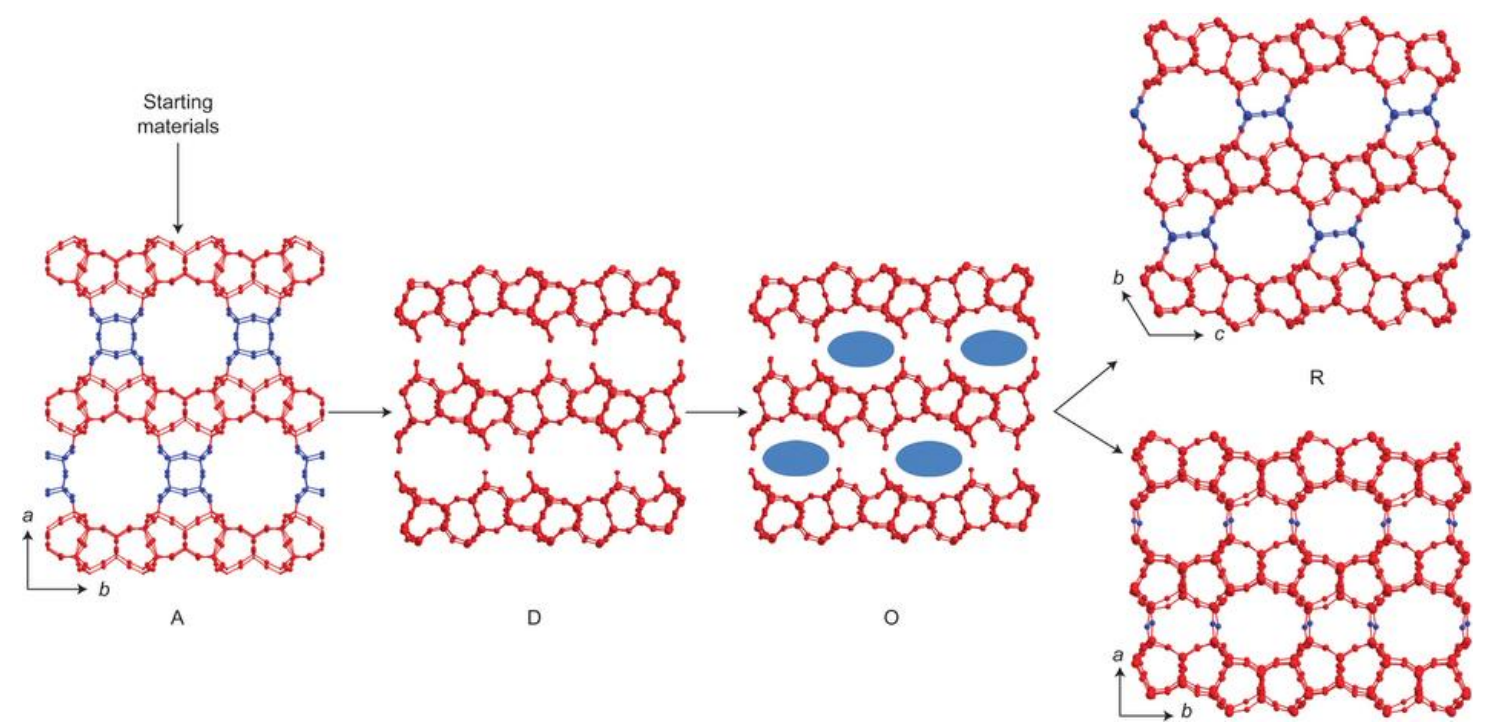

Figure 4.2.2 : ADOR strategy for the synthesis of zeolite structures with PCR and OKO topologies (R) starting from the UTL structure (A). [reprinted with permission of ref. ${ }^{114}$ ].

A wide spectrum of experimental and computational techniques has been used to obtain deep insight into this complex problem. Computational methods initially included cluster and periodic DFT optimizations, which were later extended with periodic frequency calculations to add temperature effects to the framework. Apart from DFT, classical force fields were also employed to provide a flexibility analysis of the framework. First of all, the position of the germanium atoms in the parent material is of high importance for both mechanisms. Moreover, a thorough understanding of the interactions between the separate layers and their arrangement with respect to each other is desired. Finally, the reversible transformation from the periodically interrupted to the fully connected framework is a unique feature where theoretical calculations can shed some light on the phenomena that are at play.

First of all, the position of germanium in the germanosilicate UTL zeolite determines the stability of the parent material and the possibility to convert it into the (-)OKO topology. Acid leaching requires that germanium is located in a four-ring parallel to the UTL layers, that can be dislodged selectively from the structure. In the ADOR approach, the double four-ring connecting the layers is even disassembled entirely, so germanium is preferably not present in the layers to ensure their structural stability. ${ }^{115}$

The synthesis of zeolites containing double four-membered ring (D4R) structures depends critically on the presence of $\mathrm{Ge}$ in the synthesis mixture and several theoretical studies have shown Ge atoms are the most stable in D4R positions. ${ }^{116,117}$

The fluoride synthesis route is the most commonly used method to fabricate Si/Ge zeolites. The role of ${ }^{19} \mathrm{~F}$ NMR to resolve the framework structure is indispensable, and here theory may assist 
experimentalists in rationalizing the signals arising from the different chemical compositions of the D4Rs. We refer to the NMR spectroscopic section (section 5.2) for further details about the role of germanium in the D4R units.

The stability of Ge at different crystallographic positions in the periodic framework structure is an interesting issue that has attracted attention from theoreticians. Sastre et al. ${ }^{118}$ found a linear correlation between the Si-O-Ge angle and the energy of the framework for AST, ASV, BEA, BEC, ISV and LTA zeolites using a force field approach. Low Si-O-Ge angles are therefore energetically favored, explaining the preferential inclusion of Ge in the D4R. Kamakoti et al. used DFT calculations to demonstrate for BEC that Ge is not only more stable in the D4R, it is also preferably clustered in a four-ring. This is the case for loadings from 4 up to $8 \mathrm{Ge}$ atoms per unit cell. ${ }^{119-121}$ Next to the position of Ge atoms, the structural stability of the layers and the interaction between consequent layers is also of critical importance. In the ADOR mechanism to transform UTL to (-)OKO, the layers are first organized and then reassembled into the final framework. Several computational methods have been tested to describe the interlayer interactions. Periodic DFT calculations with different functionals were benchmarked against coupled-cluster calculations of a 3T cluster interacting with a 1T-site. ${ }^{122}$ The non-local exchange-correlation functional vdW-DF2 gives an excellent agreement for the interaction energy between the layers. Other DFT methods (optB86b, optPBE and PBE-D2) overestimate the interlayer interaction by $10-20 \%$. The modified ClayFF force field follows the vdW-DF2 layer dissociation curve closely.

The study shows that the interlayer hydrogen bonds, formed between the surface silanol groups, are responsible for $80 \%$ of the interaction between layers. The other $20 \%$ stems from dispersion interactions. As a result, the interlayer arrangements with the largest number of hydrogen bonds are therefore also the most stable. ${ }^{122}$ Further modeling of the organization phase enabled to predict 20 hypothetical layered zeolites that can be obtained by conversion of existing zeolites like UTL. ${ }^{115}$ There are more computational studies on layered and two-dimensional zeolites, but to stay focused on the interconversion of zeolites, we refer to the original manuscripts for detailed reading. ${ }^{113,123,}$ 124

A last point in this section on the interconversion of zeolites is the reversible opening and closing of the (-)COK-14 framework, the bottom part in Figure 4.2.1. Experiments find that the systematically interrupted -COK-14 is the product obtained after acid leaching of Germanium. Calcination at temperatures above $700 \mathrm{~K}$ is necessary to condense the silanol groups and obtain the fully connected COK-14 framework. It is possible to hydrolyze the latter framework reversibly, by immersing the framework in warm water or by leaving it to ambient atmosphere for an extended period of time. ${ }^{112}$ 
Static DFT calculations at OK can no longer describe this temperature- and time- dependent behavior. The electronic energy profile at $0 \mathrm{~K}$ shows that the systematically interrupted (open) framework is more stable than the fully connected (closed) one (Figure 4.2.3). Full vibrational analysis is needed to determine the thermal contribution to the enthalpy and the entropy of the reaction. When temperature is taken into consideration, it is clear that entropy is the driving force and that the Gibbs free energy of the closing is much more negative at $700 \mathrm{~K}$ than at $300 \mathrm{~K}$. This is mainly due to the release of $4 \mathrm{H}_{2} \mathrm{O}$ molecules upon condensation of the silanol groups.

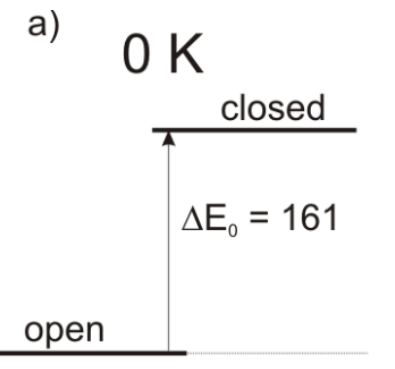

b)

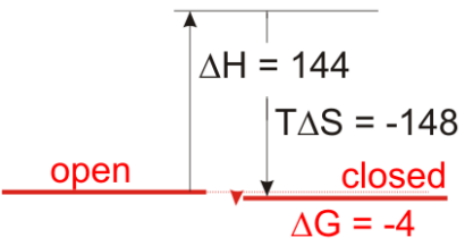

c) $700 \mathrm{~K}$

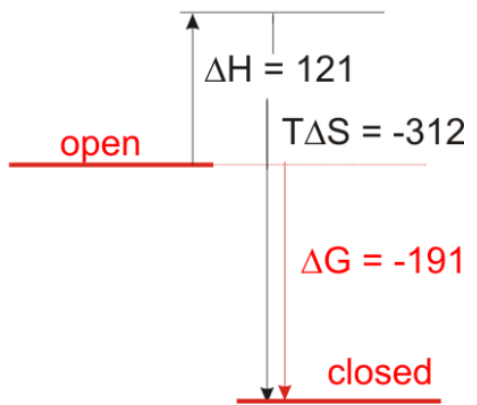

Figure 4.2.3 : Electronic energy and free energy profile for the open and closed COK-14 framework. Energies are in $\mathrm{kJ} \mathrm{mol}^{-1}$. [reprinted with permission of ref. ${ }^{112}$ ]

To obtain a framework without imaginary frequencies, the framework was not only optimized with respect to the forces, but also directed towards the minimum by careful screening of the normal modes. To do so, the framework was relaxed first, after which a frequency calculation was performed. If there are imaginary frequencies in the framework, then the atoms were moved in the direction of the vectors corresponding with these normal modes. Afterwards, the atoms were geometrically relaxed again and the procedure was repeated until there are no longer imaginary modes. A strategy to do this more systematically is proposed by Sauer and coworkers. ${ }^{125}$ The theoretical description of such chemical and physical transformations of lattices, poses an interesting challenge for future methodologies that account for both flexibility of the lattice and anharmonic effects. A possible direction is the usage of advanced MD methods that would account properly for all entropy effects. A careful analysis of the flexibility of the systematically interrupted versus fully connected framework by considering the range of densities within which the atoms in the framework are stress-free, gives another interesting view on this complex problem. Indeed such analysis reveals that the fully connected COK-14 framework is too rigid in the pure silica form, but could exist when enough heteroelements such as Ge or Al are introduced, which is confirmed by 
experiments. ${ }^{105,126,127}$ More details on the flexibility window of zeolites were given in the previous section.

\subsection{Modelling the external surfaces of zeolites}

Little attention has in general been paid to the structures of the external surfaces of zeolites as catalytic action and sorption generally involve the internal pores of the materials. However, external surfaces may control access to the internal pore structure and may strongly influence crystal morphology. Crystal growth involves surfaces processes and the value of modelling in this context was shown in section 3.2. Surface modelling tools are well developed in materials simulation and may use both interatomic potential and electronic structure techniques. In the former case, the commonest procedure is to use a "two-region" approach with 2-dimensional periodic boundary conditions, in which the surface and a region comprising several layers below are fully relaxed using minimization methods with this relaxed region embedded in a rigid representation of the underlying lattice. Electronic structure calculations generally use a slab model (as was the case in the calculations discussed in section 3.2 ), which is repeated infinitely in the $3^{\text {rd }}$ dimension so that standard 3-D periodic methods and codes can be employed. The slab is then wholly or partially relaxed.

Both approaches have been applied in the modelling of zeolite external surfaces. An example of the former is the work of Whitmore, Slater et al. ${ }^{128}$, who modelled the external surfaces of zeolite $Y$ using interatomic potential based methods. Earlier simulation work ${ }^{129}$ had established that the hydroxylated <111> surface was the dominant low energy external surface of the material. This study extended the earlier predictions by investigating the adsorption of benzene on the $<111>$ surface. Three distinct adsorption sites were revealed as illustrated in Figure 4.3.1.
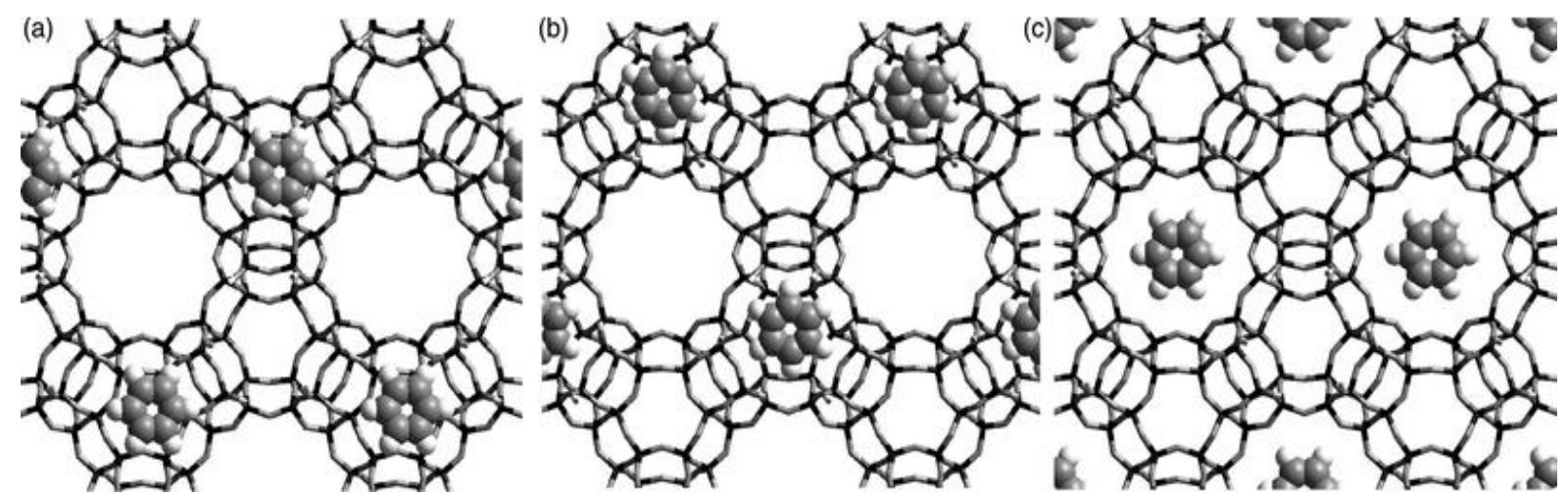

Figure 4.3.1 (a) External surface $6 \mathrm{MR}$ adsorption of benzene; (b) external surface half SOD cage adsorption of benzene; (c) external surface $12 \mathrm{MR}$ adsorption of benzene. [taken with permission from reference ${ }^{128}$ ] 
The site over the $6 \mathrm{MR}$ was predicted to have the lowest energy. Moreover, the calculations suggest that there is a relatively low activation energy for the penetration of the molecule into the bulk lower than for diffusion in the bulk which may lead to surface enrichment of the sorbed molecule.

Recently the diffusion of molecules through the external surfaces of zeolites has been examined using MD and MC simulations by Zimmermann et al. ${ }^{130}$, who modelled the permeation of methane and ethane into and out of an AFI-type zeolite. Interestingly they found that diffusion paths both internal and external are close to the pore wall and that there are appreciable molecular re-crossing events at the surface. The work again illustrates the significance of processes involving external surfaces and the ability of modelling techniques to probe these in detail.

As shown earlier in this section, surface modeling has also proved of considerable value in modeling factors related to crystal growth and their utility in zeolite science is clearly established.

\section{Computational spectroscopy for characterization in zeolite chemistry}

Computational spectroscopy has evolved drastically in latest years, and its use as a characterization tool in zeolite chemistry is nowadays widely accepted. Early research focused on the use of small model systems for zeolite materials and possible reaction intermediates were analyzed without accounting for realistic process parameters, i.e. characterization at $0 \mathrm{~K}$. Nowadays, in-situ experiments deliver data which evolve in time, which vary with temperature and other operating conditions. Molecular simulations have now reached such a level of accuracy that they are able to reproduce the experimental data under real operating conditions. ${ }^{131}$ In-situ spectroscopic techniques aim to reveal the real nature of active sites of the catalyst materials in modus operandi. This information is crucial to decipher the key stages of reaction processes. The field of operando spectroscopic techniques has undergone a tremendous evolution in the last decade, noteworthy are the advances in in-situ IR and optical techniques. ${ }^{132}$ Dedicated complementary spectroscopy tools have been developed to tackle various length and time scales involved in the realistic heterogeneous catalytic systems. Modern spectroscopic characterization studies usually involve a large set of complementary techniques. In this review, we select three main spectroscopic techniques (Infrared, NMR and optical spectroscopy) and describe recent evolutions in their theoretical description. In particular, IR and NMR spectroscopy will be reviewed, focusing on the latest trends mainly due to an improved description of the zeolite models and use of MD. The breakthrough of the time-dependent form of DFT, i.e. TD-DFT, has also enabled the study of electronic excited states of relatively large systems and hence the 
computation of excitation and emission spectra becomes possible. It has opened a lot of perspectives from the theoretical side to give insight in the interpretation of experimental spectra as measured in UV/Vis and luminescence spectroscopy.

\subsection{Infrared spectroscopy}

Infrared (IR) spectroscopy can cover the whole area of catalyst characterization, probing transitions between 4000 and $10 \mathrm{~cm}^{-1}$. It can expose the geometric structure of the solid zeolite material and its changes in lattice vibrations and/or acoustic modes, the chemical structure of adsorbed molecules and host-guest interactions via hydrogen and coordination bonds. Hence, IR spectroscopy can provide crucial insights on zeolite formation, growth and adsorption of small intermediates, forming an essential characterization tool in zeolite chemistry. Increasing computing power and clever implementations have led to breakthroughs in the field of computational IR spectroscopy.

Accurate calculation of IR spectra has long been a difficult task, since it requires calculation of the computationally demanding second order derivatives of the total energy. The computation of these derivatives for complex structures has been facilitated by devoted techniques treating parts of the zeolite structures as blocks. ${ }^{133,134}$ The accurate computation of soft, low frequency modes characteristic of molecule-surface interactions - cannot be achieved in an harmonic approach, as these modes show strong anharmonicities. Inaccurate frequencies lead to unphysical predictions for adsorption entropies and free energies, leading to large deviations with experiment. Noteworthy, Piccini and Sauer implemented a computational protocol based on normal mode coordinates - instead of Cartesian coordinates - for structure optimization and for obtaining harmonic frequencies by numerical differentiation. This method was very useful when employing plane wave basis sets as in periodic DFT calculations. ${ }^{125,135}$ Anharmonic corrections are obtained using a fourth-order expression in harmonic normal coordinates for the Hamiltonian. This method was applied to investigate adsorption of methane, ethane and propane in $\mathrm{H}-\mathrm{CHA}$ and induced changes in free energies of adsorption varying from $+4.3 \mathrm{~kJ}$ $\mathrm{mol}^{-1}$ (ethane) to $-6.6 \mathrm{~kJ} \mathrm{~mol}^{-1}$ (propane) systematically improving the experimental agreement. ${ }^{125,135}$ This type of studies assists in a continuous process of predicting more accurate frequencies and these procedures will become more routine procedures in the near future.

It is not our intention to review the large number of IR studies employing computational simulations for characterizing reaction intermediates of different sorts of zeolite-catalyzed reactions. Instead, we focus on two particular items: (1) theoretical IR spectra of zeolites via MD methods and (2) theoretical shifts in IR frequencies using $\mathrm{CO}$ as a probe molecule to assess the acidity of the zeolite catalyst material. 


\subsubsection{Theoretical IR spectra of zeolites via molecular dynamics methods}

The zeolite lattice dynamics and its characteristic vibrational frequencies can be investigated using MD simulations. This methodology offers advantages over the traditional Normal Mode Analysis (NMA), accounting for the anharmonicity of the potential energy surface and allowing sampling of multiple minima. Various studies have been devoted to the interpretation of vibrational spectra of zeolite catalysts in terms of structural entities. From the time-dependent data, infrared spectra can be obtained via the Fourier transformation of the dipole moment auto-correlation function $\langle d \bar{\mu}(0), \bar{\mu}(t)\rangle$, resulting in the following expression for the IR adsorption cross section : $:^{136}$ $\alpha(\omega) \sim \lim _{\tau \rightarrow \infty} \frac{1}{\tau}\left|\int_{0}^{\tau} d t e^{-i \omega t} \frac{d \bar{\mu}(t)}{d t}\right|^{2}$.

Velocity power spectra can be obtained since the time derivative of the dipole moment can be approximated as:

$$
\frac{d \bar{\mu}(t)}{d t}=\sum_{i=1}^{N} q_{i} \bar{v}_{i}(t)
$$

with $q_{i}$ the charge and $v_{i}$ the velocity of the atom $\mathrm{i}$ and $\mathrm{N}$ the number of atoms.

Smirnov and Bougeard investigated zeolite frameworks constructed from sodalite cages, in particular the SOD, LTA and FAU topologies. In an initial study, comparison of two force fields indicated that a simplified general valence force field - omitting long-range and cross terms resulted in IR and Raman spectra in good agreement with experiment. ${ }^{137}$ The same force field was further used to examine in detail the contribution of the 4- and 6-rings of the SOD cage in the various frameworks, using the autocorrelation of the breathing coordinate of an individual ring. In line with an earlier study of de Man and van Santen ${ }^{138}$, the authors conclude that no general correlation could be found between the pore-opening vibration of the ring structures with the infrared spectra of zeolite spectra. ${ }^{139}$

On the other hand, using larger models representing zeolite subunits at particular stages of the growth process, Lesthaeghe et al. were able to elucidate the origin of the characteristic MFI fingerprint band around $550 \mathrm{~cm}^{-1} .{ }^{140} \mathrm{An}$ in-house developed force-field for zeolites, based on the gradient curves method (GCM) , was employed. ${ }^{141}$ Model systems of increasing size (from 5 to 432 Si atoms) representing the elementary 5-rings up to a true nanoslab were constructed and their vibrational spectra were simulated. It was shown that while isolated five-membered rings show characteristic vibrational bands around $650 \mathrm{~cm}^{-1}$ the combination of five-membered rings in the MFI- 
type structure (modeled using a 1D half nanoslab, 2D nanoslabs and 3D nanoblocks) readily generates the bathochromic shift to the typical pentasil vibration around $550 \mathrm{~cm}^{-1}$ (see Figure 5.1.1). This IR band does not shift gradually with increasing nanoparticle size, but highly depends on the specific way structural units are added.

a)

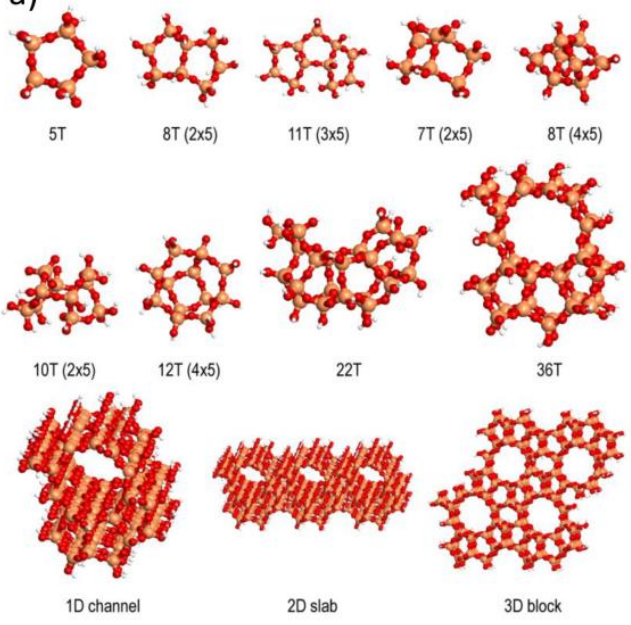

b)

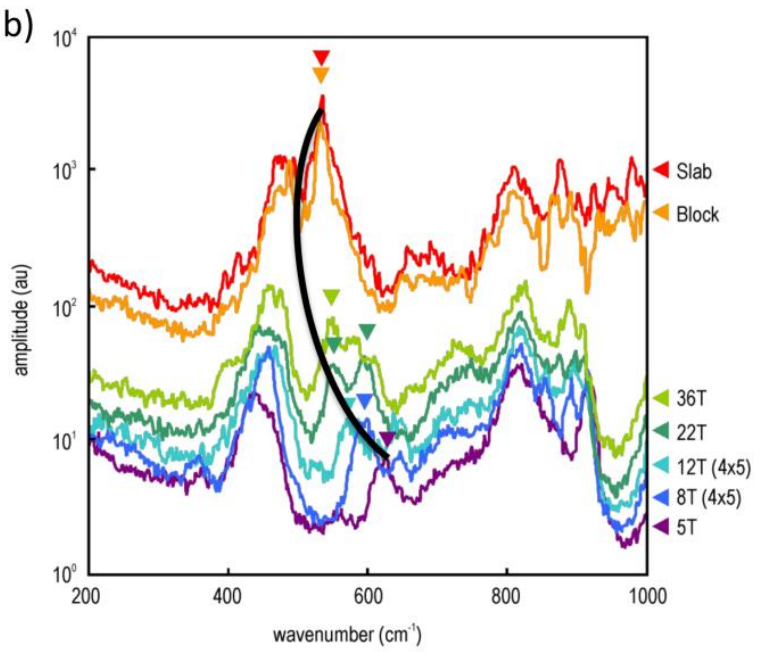

Figure 5.1.1 : (a) Models of increasing size, ranging from the isolated five ring (5T), to the $3 \times 4$ silicalite nanoslab (slab), and $2 \times 2 \times 2$ nanoblock (block). (b) Simulated IR spectra. Adapted with permission from ref. ${ }^{140}$.

In a recent work, Bueno-Perez et al. examined the use of force field models for their ability to compute reliable IR spectra of different zeolite frameworks. ${ }^{142}$ Three force fields - developed by Nicholas et al. ${ }^{143}$, Demontis et al. ${ }^{144}$, and Hill and Sauer ${ }^{145}$ - were tested by calculating IR spectra of 9 flexible frameworks. Overall, the Nicholas force field performed best when comparing with experimental spectra on quantitative basis. The various spectra were compared after introducing a similarity index. Using this similarity analysis, the nine frameworks could be - related to their topology - classified in four sets. Noteworthy, this classification based on theoretical data does not agree with the one obtained using experimental spectra. Despite the detailed vibrational information obtained in this work, the authors concluded that the transferability still poses a main problem.

MD simulations involving classical potential models have also been used to study the vibrational behavior of cation-exchanged zeolites. The low-frequency vibrations of extra-framework cations in mordenite zeolites were studied by Maurin, Bell and co-workers. ${ }^{146}$ For a recent review on the use of interatomic potentials for small pore silica zeolites, we refer to ref. ${ }^{147}$. Also in this study, the 
influence of the particular force field was discussed, reporting changes in frequencies and overall shape of the spectra for Na-exchanged MOR. The framework Si/Al ratio, local environment and site symmetry of the cations also turned out to influence the theoretical power spectra. Furthermore, the series of $\mathrm{Na}^{+}, \mathrm{K}^{+}, \mathrm{Mg}^{2+}$ and $\mathrm{Ca}^{2+}$ cations in MOR was compared. The evolution of the frequency (see Figure 5.1.2) could be explained using their mass and charge, the up-shift induced by the charge was found to be dominant.

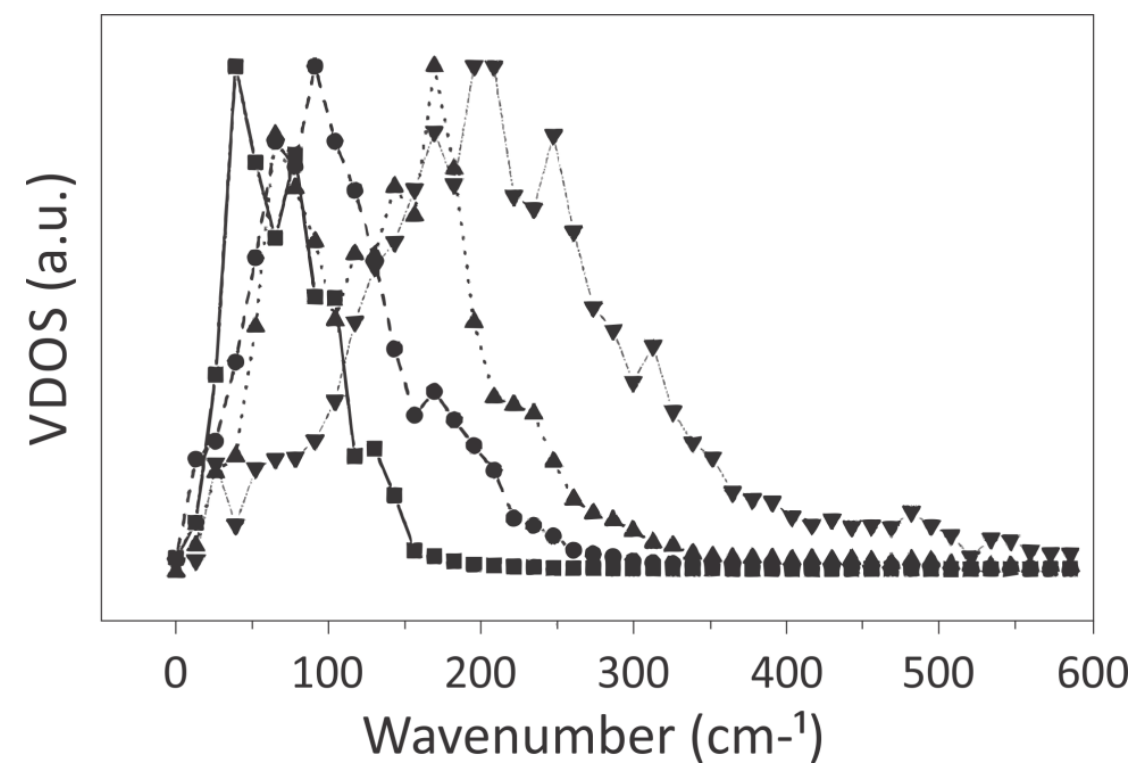

Figure 5.1.2. Evolution of the vibrational power spectrum as a function of the nature of the alkali and alkaline earth extra-framework cations $\mathrm{K}+$ (solid line with square symbols), $\mathrm{Na}+$ (dashed line with circle symbols), $\mathrm{Mg} 2+$ (dotted line with up-triangle symbols) and $\mathrm{Ca} 2+$ (dashed-dotted line with down triangle symbols). Reprinted with permission from ref. ${ }^{146}$.

Previous studies demonstrate that force fields are the natural choice to perform MD simulations on zeolite systems, mainly because they are able to sample during a representative time frame ( ns range). ${ }^{137,139,143-145,148} \mathrm{It}$ is nevertheless clear that careful benchmarking is mandatory when employing these classical techniques to describe the vibrational behavior from dynamic simulations. Increasing computing facilities hence open the route to perform dynamic simulations based on quantum mechanical equations of motions. In the field of zeolite chemistry, only a few studies report on vibrational spectra derived from ab initio MD (AIMD) simulations. In these particular cases, advanced analysis schemes are developed to unravel the vibrational spectra of zeolitic building blocks. ${ }^{149,} 150$ AIMD simulations and subsequent derivation of IR spectra can add insights obtained with computational spectroscopy. It has the main advantage that the time-consuming development of a force field particularly designed for the envisaged zeolite and its specifications is no longer 
mandatory; nevertheless the obtained results depend on the applied methodology and approximations made in the QM description and should be validated as well.

\subsubsection{Theoretical shifts in the IR frequencies using $\mathrm{CO}$ as a probe molecule to assess the acidity of the zeolite catalyst material}

In the context of zeolite characterization using IR spectroscopy, the use of probe molecules is a popular technique. Adsorption of base molecules, such as $\mathrm{H}_{2}, \mathrm{CO}, \mathrm{NH}_{3}, \ldots$, can address the acidity of the zeolite material and/or reveal information on the actual acid sites present throughout the material. This field has grown enormously in the latest decades and the reader is referred to extensive reviews entirely devoted to this topic. ${ }^{151-158}$ Adsorption of small probe molecules is an indirect technique, revealing information on the characteristics of the catalyst via changes in the frequencies of the zeolite hydroxyl group and of the probe molecule. Herein, we highlight the main findings related to $\mathrm{CO}$ adsorption in zeolite materials that have been obtained using theoretical simulations. Carbon monoxide is an archetypal probe used for zeolite characterization, the stretching mode of this very weak base is highly sensitive to its local environment. The small size of the molecule ensures that it can access the zeolitic micropores. CO adsorption (see Scheme 5.1.1) has been used to assess the acidity of both proton- and metal-exchanged zeolites, exhibiting Brønsted and Lewis acidic character, respectively. Both C- and O-end adsorption is possible, however calculated adsorption energies of the $\mathrm{C}$-adducts are over $50 \%$ higher than those of the $\mathrm{O}$-adducts ${ }^{159}$, confirming experimental findings that the latter desorb already at low temperature. Frequency shifts are usually reported together with interaction energies and/or enthalpies; however we limit our review to the discussion of the vibrational properties.

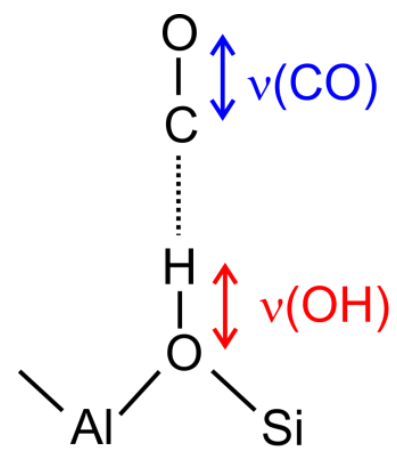

BAS

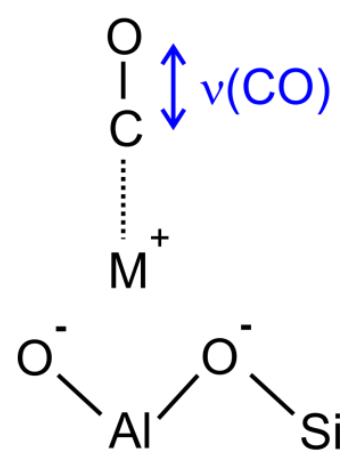

LAS

Scheme 5.1.1 : Schematic representation of $\mathrm{CO}$ adsorption (through C-end) at Brønsted (BAS) and Lewis acid sites (LAS). Relevant stretching frequencies of the zeolite $\mathrm{OH}$ group and the $\mathrm{CO}$ probe are depicted. 
Derouane and co-workers recently reviewed this topic, pointing out that characterization of the acidity of zeolite materials is not straightforward since many factors play a role. ${ }^{160}$ Main factors are that Brønsted (BAS) and Lewis acid (LAS) sites can be present at the same time, their acid strength can vary continuously in a broad range and defects can modify the acidity to a large extent. Simulation of changes in vibrational frequencies is yet one of the most simple and unambiguous methods that can be applied. Scheme 5.1.1 illustrates that $\mathrm{CO}$ interacts with hydroxyl acid groups via hydrogen bonding and with cationic acid sites mainly through electrostatic interactions, leading to a blue shift of the $v(\mathrm{CO})$ stretching frequency. In case of adsorption at BAS, red shifts in the hydroxyl stretching frequency $v(\mathrm{OH})$ can also be computed and used as an indicator of interaction strength. These simulated vibrational frequencies largely depend on two factors, in particular the level of theory and the applied model for the zeolite catalyst. Nowadays, it is accepted that special care needs to be taken to include dispersion corrections into the applied methodology and that the structural models need to be large enough to account for the zeolite environment.

Initial studies employed small cluster models to mimic the zeolitic acid site, as reviewed by Sauer et al. in $1994{ }^{161}$. Larger clusters, accompanied with suitable embedding schemes have been applied extensively and proven to provide accurate results when the inner cluster is taken sufficiently large, see e.g. refs ${ }^{162,163}$ for $\mathrm{CO}$ adsorption at $\mathrm{Cu}(\mathrm{I})$-exchanged zeolites.

In the context of proton-exchanged zeolites, adsorption of $\mathrm{CO}$ and shifts in stretching frequencies of both the zeolite $\mathrm{O}-\mathrm{H}$ and adsorbed $\mathrm{C}-\mathrm{O}$ have been frequently computed. For example, calculated frequency shifts of the isostructural H-SAPO-34 and H-SSZ-13 were shown to indicate the enhanced acidity of the alumino silicate material. In particular, adsorption of $\mathrm{CO}$ was considered at finite extended (42 T sites) clusters, scaled B3LYP-based frequencies lead to a frequency shift of -311 and $364 \mathrm{~cm}^{-1}$ for the zeolite hydroxyl group of H-SAPO-34 and H-SSZ-13, respectively. ${ }^{164}$ These values overestimate the experimentally reported values of -270 and $-316 \mathrm{~cm}^{-1} 165$, but the relative trend is in good agreement.

Noteworthy, Arean et al. recently emphasized that direct correlation of DFT-computed $v(\mathrm{OH})$ with acid strength might not always be possible, particularly when dealing with zeolites with different structure type. ${ }^{166}$ Further studies are necessary to investigate this topic.

Similar comments should be made for the study of metal-exchanged zeolites. IR spectra of CO adsorbed on alkali metal cations in various zeolites appear to be rather different. They differ in 
number of distinguishable bands and in the spectra dependence on the $\mathrm{CO}$ coverage. The group of Nachtigall et al. demonstrated the strength of theoretical simulations to characterize the sitespecific CO-zeolite adsorption complexes responsible for the various peaks in experimental IR spectra. First of all, theoretical simulations are crucial to determine the cation location. For this goal, periodic calculations are clearly preferred over cluster calculations. ${ }^{167}$

CO adsorption at various metal-exchanged zeolites - in particular, ZSM-5, FER and A ${ }^{162,168-181}$ - was investigated using theoretical methods. Noteworthy, a scaling method based on the correlation between the $\mathrm{CO}$ bond length and the $\mathrm{CO}$ stretching frequency - and hence denoted the $\omega / r$ correlation method - was developed, leading to computational frequencies in excellent agreement with experimental values. This method extends beyond the use of scaling of the harmonic frequencies using a fixed scaling parameter ${ }^{182,}{ }^{183}$, which is still considered a very popular and useful technique. Using the $\omega / \mathrm{r}$ correlation method, frequencies are evaluated using : ${ }^{184}$ $\omega_{C O}=a r_{C O}+b+\Delta v+\Delta \omega$,

Using the equilibrium CO distance $r_{C O}$ and an anharmonicity correction $\Delta v$. This correction was e.g. $29 \mathrm{~cm}^{-1}$ in the case of adsorption at FER-exchanged zeolites. The basis-set dependent parameters a, b and $\Delta \omega$ were obtained from $\operatorname{CCSD}(T)$ calculations on a set of test molecules adsorbed at an $1 T$ cluster. The main advantage of this method is that it omits the construction of the Hessian matrix for the adsorbant/zeolite system, since computation of the $\mathrm{CO}$ bond length is sufficient. Using this method, vibrational frequencies of adsorbed CO can be calculated with near-spectroscopic accuracy.

PBE-based calculations on M-FER zeolites ( $\mathrm{M}=\mathrm{Na}, \mathrm{K}, \mathrm{Li})$ were able to unravel the experimental spectra. Both mono- and di-carbonyl species contribute to the IR peaks. Moreover, the theoretical data also demonstrate the importance of dual site adsorption. Figure 5.1.3 (a) depicts CO complexes on $\mathrm{K}+$ dual sites in FER, responsible for the IR peak at $2148 \mathrm{~cm}^{-1}$ in Figure 5.1.3(b). The other peaks of Figure 5.1.3 (b) were assigned to C-end $\left(2163 \mathrm{~cm}^{-1}\right)$ and O-end $\left(2116 \mathrm{~cm}^{-1}\right)$ absorption of CO on a single metal site, respectively. ${ }^{179}$ 

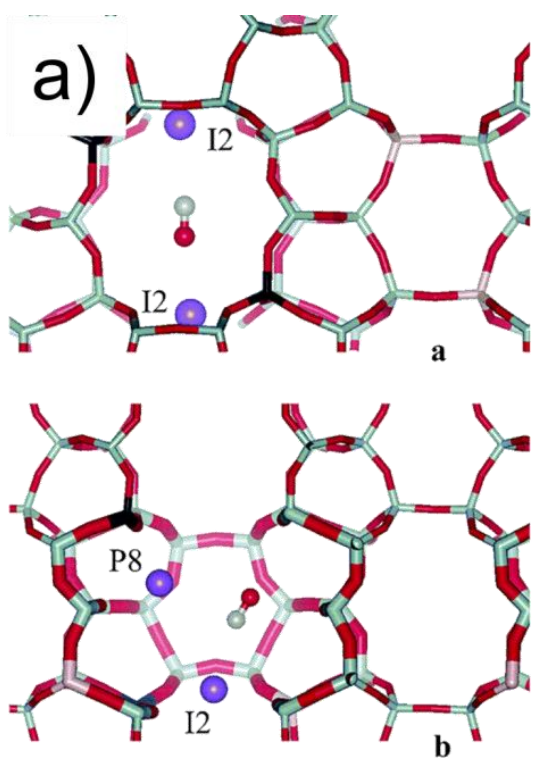

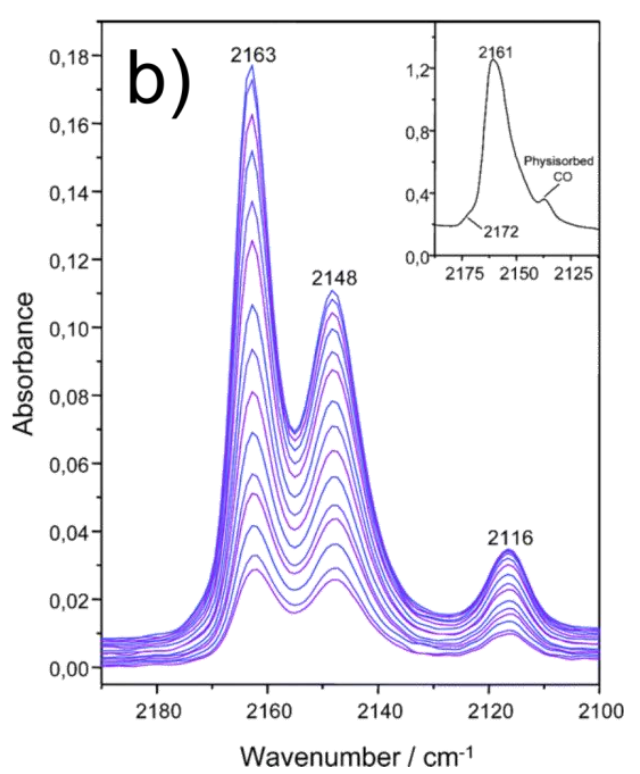

Figure 5.1.3. (a) CO complexes on $\mathrm{K}+$ dual sites for two locations. (b) Variable-temperature FTIR spectra (zeolite blank subtracted) of $\mathrm{CO}$ adsorbed on K-FER, Si: Al=27.5:1. Temperature goes from 176 to $223 \mathrm{~K}$, and pressure from 0.12 to 0.64 Torr. Adapted with permission from Ref. ${ }^{179}$

Using periodic ab initio simulations with the software package VASP, the group of Hafner et al. contributed substantially to an improved theoretical description of $\mathrm{CO}$ adsorption in zeolites, in particular in MOR ${ }^{159,185,186}$ and $\mathrm{CHA}{ }^{187}$. Simulations on distinct adsorption sites can distinguish between high- and low-frequency bands. Figure 5.1.4(a) displays calculated C-O stretching frequencies as a function of the calculated $\mathrm{C}-\mathrm{O}$ distances for $\mathrm{CO}$ adsorption on different active sites in MOR. These values were obtained from MD simulations on the periodic cells using the GGA PW91 functional. ${ }^{159}$ In Figure 5.1.4(a), the stretching frequency of gas phase $\mathrm{CO}$ is also depicted, and comparison with the experimental value of $2140 \mathrm{~cm}^{-1}$ hints towards an accuracy of the simulations (i.e. overestimation of 12 $\mathrm{cm}^{-1}$ comparing the theoretical and experimental $v(\mathrm{CO})$ value). In another study, these authors pointed out the need for large clusters and/or periodic models for the zeolite structure and concluded that the RPBE functional provides a better description of the hydrogen-bonded systems, leading to red-shifts of the zeolite hydroxyl group in close agreement with experiment. ${ }^{185}$ 

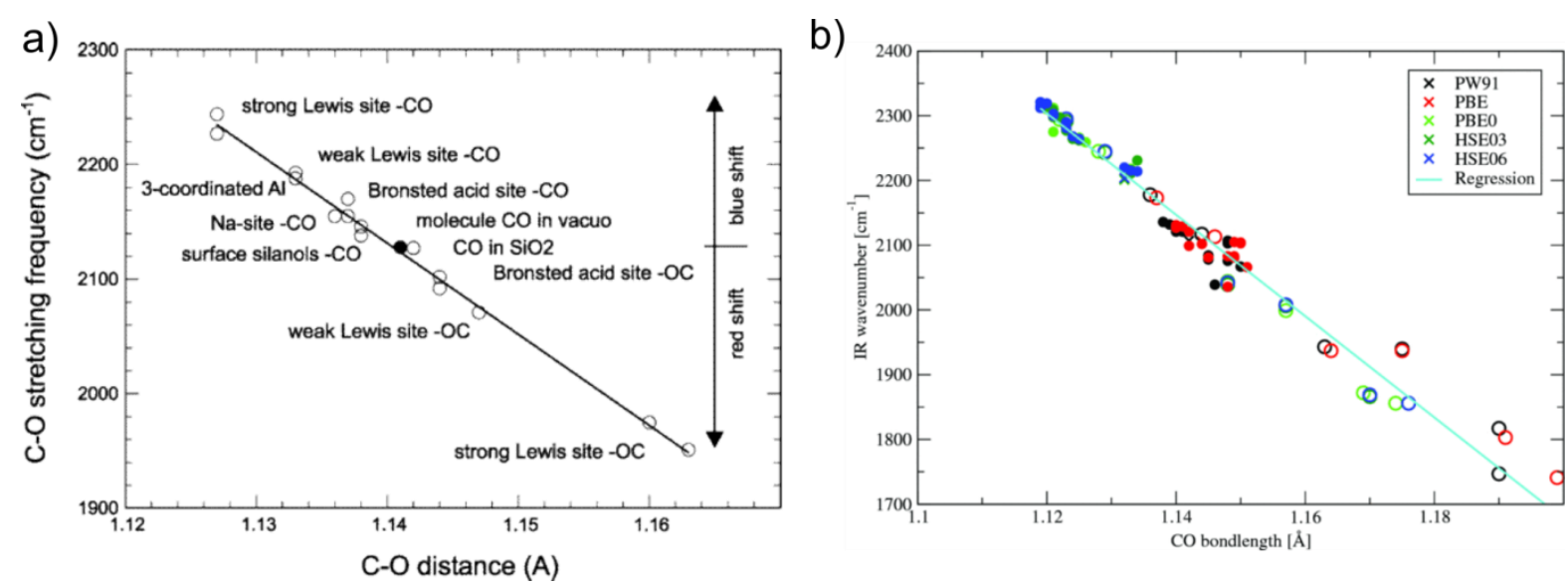

Figure 5.1.4 : Calculated stretching frequencies of $\mathrm{CO}$ versus $\mathrm{CO}$ distance. (a) Results for distinct adsorption sites in MOR. Calculated from MD simulations using the PW91 functional. Reprinted with permission from ref. ${ }^{159}$ (b) Results for $\mathrm{Cu}(\mathrm{I}), \mathrm{Cu}(\mathrm{II})$ and $\mathrm{Co}(\mathrm{I}) \mathrm{CHA}$ zeolites. Calculated using various DFTbased functionals. ${ }^{187}$ Adapted with permission from Ref. ${ }^{187}$.

Progress has also been made in the theoretical description of $\mathrm{CO}$ adsorption on cations of noble and transition metals. In this case, the CO frequency is shifted downwards; the interaction is dominated by $\pi$-back-donation from the metal to the CO molecule. Initial studies reported on the failure of DFT to predict this blue-shift of the CO stretching mode. Dedicated computational work by Goltl and Hafner addressed CO adsorption at cupper- and cobalt-containing CHA zeolites. ${ }^{187}$ Both GGA- PW91, PBE and hybrid -PBEO, HSE03, HSEO6 - DFT methods were investigated. The calculated $v(\mathrm{CO})$ shifts versus CO bond distances are depicted in Figure 5.1.4(b). The full dots correspond with the value for the adsorption complexes. The results indicate that overall a linear trend is maintained, and that only the hybrid functionals correctly predict a blue shift of the $\mathrm{CO}$ stretching frequency. Further studies employed this information for calculating IR spectra of Cu-SSZ-13 used as catalyst in DeNOx chemistry (see section 6.4). ${ }^{188}$ These studies extend beyond the $\omega / \mathrm{r}$ scaling method, which also showed successful for CO adsorption at $\mathrm{Cu}(\mathrm{I})$-exchanged MFI zeolites. ${ }^{162}$ These authors concluded that QM-Pot simulations with sufficiently large inner parts - in particular a minimal inner part of $16 \mathrm{~T}$ atoms - also result in accurate values of the stretching frequencies, as listed in Table 5.1.1.

Table 5.1.1: Theoretical $\mathrm{CO}$ bond lengths [in $\mathrm{A}]$ and stretching frequencies $\left[\right.$ in $\left.\mathrm{cm}^{-1}\right]$ upon adsorption at $\mathrm{Cu}(\mathrm{I})$-exchanged MFI obtained at the QM-Pot and Periodic DFT Levels. All details can be found in ref. ${ }^{162}$. 


\begin{tabular}{llll} 
& DFT functional & $\begin{array}{l}r(\mathrm{CO}) \\
\AA\end{array}$ & $\begin{array}{l}\mathbf{v}(\mathrm{CO}) \\
\mathrm{cm}^{-1}\end{array}$ \\
\hline QM-pot 1T & BLYP & 1.1487 & 2137 \\
QM-pot 3T & BLYP & 1.1487 & 2142 \\
QM-pot 6T & BLYP & 1.1484 & 2138 \\
QM-pot 8Td & BLYP & 1.1478 & 2142 \\
QM-pot 16T & BLYP & 1.1449 & 2158 \\
QM-pot 17T & BLYP & 1.1447 & 2159 \\
QM-pot 28T & BLYP & 1.1458 & 2153 \\
\hline Periodic & PBE & 1.1500 & 2154 \\
Periodic & PW91 & 1.1486 & 2154 \\
\hline
\end{tabular}

\subsection{NMR spectroscopy}

Since the development of magic angle spinning (MAS) for solid-state NMR, this technique has played an important role in zeolite characterization. This technique has currently reached such a high level of resolution that it becomes indispensable for structure determination. In most cases MAS NMR is considered as complementary to other characterization techniques like diffraction. These experimental techniques together with first-principles calculations, form a multi-technique approach, which more and more becomes the norm for a full characterization investigation. The basic constituents of a NMR study are the NMR-active species : ${ }^{29} \mathrm{Si},{ }^{27} \mathrm{Al}$ and ${ }^{17} \mathrm{O}$ for silica zeolites and ${ }^{31} \mathrm{P},{ }^{27} \mathrm{Al}$ and ${ }^{17} \mathrm{O}$ for aluminophosphates (AIPOs). In addition, charge-balancing cations and several structure directing agents (SDAs) may also contain NMR-active elements. It makes solidstate NMR a powerful tool for structure determination, but it also generates a wealth of peaks in the spectra, and first-principles calculations are here needed to introduce some order in the jungle. To exploit the inherent periodicity of these zeotype frameworks, periodic codes are requisite in which a NMR module has been implemented. To the most frequently used program packages, used in literature, belong CASTEP ${ }^{189}$, which employs the GIPAW algorithm ${ }^{190}$ to reconstruct the allelectron wave function in the presence of a magnetic field and VASP (Vienna Ab initio Simulation Package) ${ }^{191,192}$, also employing the GIPAW algorithm ${ }^{190}$ and using projector-augmented waves and a plane wave basis set to describe the electron density. Another approach is based on the Gaussian and augmented-plane-wave (GAPW) density functional method as implemented in QUICKSTEP/CP2K. ${ }^{193}$ The approach relies on the linear response (small perturbation) of a molecular 
system induced by an external magnetic field. ${ }^{194}$ Calculations generate the absolute shielding tensor $\overline{\bar{\sigma}}$ in the crystal frame. From the principal components of the symmetric part of the shielding tensor it is possible to generate the isotropic shielding, $\sigma_{\text {iso }}=(1 / 3) \operatorname{Tr} \overline{\bar{\sigma}}$. The isotropic chemical shift, $\delta_{\text {iso, }}$, is given (assuming $\left.\sigma_{\text {ref }} \ll 1\right)$, by $-\left(\sigma_{\text {iso }}-\sigma_{\text {ref }}\right)$, relative with respect to a reference substance, usually a molecule with barely distorted electron distribution. The reference compounds of relevance in solid-state NMR are: $\left(\mathrm{CH}_{3}\right)_{4} \mathrm{Si}$ for ${ }^{1} \mathrm{H}$ and ${ }^{29} \mathrm{Si}$ chemical shifts, $\mathrm{Al}\left(\mathrm{NO}_{3}\right)_{3}$ for ${ }^{27} \mathrm{Al}, \mathrm{H}_{3} \mathrm{PO}_{4}$ for ${ }^{31} \mathrm{P}, \mathrm{CFCl}_{3}$ for ${ }^{19} \mathrm{~F} \mathrm{NMR}$, etc.

\section{Application of the multi-technique approach : aluminophosphates (AIPOs) as test case}

Aluminophosphates (AIPOs) are ideal materials to be characterized by solid-state NMR spectroscopy as they are composed with NMR-active isotopes like ${ }^{27} \mathrm{Al},{ }^{31} \mathrm{P}$ and in many cases ${ }^{1} \mathrm{H}$ and ${ }^{19} \mathrm{~F}$. In many cases AIPOs are substituted with cations such like $\mathrm{Mg}^{2+}, \mathrm{Cr}^{2 / 3+}, \mathrm{Ga}^{3+}, \mathrm{Si}^{4+}$, etc. to induce catalytic properties. These species influence the chemical and physical behavior of the material and NMR is an ideal tool to investigate the internal structure of the framework.

In this field, the group of Sharon Ashbrook ${ }^{195,196}$ has done groundbreaking research by applying a multi-technique approach. Crystal structures, derived from X-ray diffraction experiments, are used as starting models for first-principles NMR calculations. And in turn these theoretical predictions are extremely useful in unraveling the experimental solid-state NMR data. A nice example of this methodology is the study of Byrne et al. ${ }^{195}$ on $\mathrm{AlPO}_{4}-15$ material, synthesized using $\mathrm{NH}_{4}{ }^{+}$as SDA. Their multi-technique approach consists of (i) identification of the distinct Al species in $\mathrm{AlPO}_{4}-15$ by high resolution ${ }^{27} \mathrm{Al} \mathrm{NMR} \mathrm{experiments,} \mathrm{(ii)} \mathrm{unambiguous} \mathrm{assignment} \mathrm{of} \mathrm{the} \mathrm{resonances} \mathrm{in} \mathrm{both}{ }^{27} \mathrm{Al}$ NMR and ${ }^{31}$ P NMR spectra with the aid of first-principles predictions, (iii) validation of the quality of the X-ray diffraction structures as starting points for the theoretical calculations.

Reference shieldings $\sigma_{\text {ref }}$ of $549,7 \mathrm{ppm}$ and $278,3 \mathrm{ppm}$ were used for ${ }^{27} \mathrm{Al}$ and ${ }^{31} \mathrm{P}$, respectively. The structure of $\mathrm{AlPO}_{4}-15$ is shown in Figure 5.2.1a with $\mathrm{PO}_{4}$ tetrahedra and $\mathrm{AlO}_{6}$ octahedra. The channels are filled with ammonium cations and water molecules, which form both hydrogen bonds to the framework. In the asymmetric unit (Figure 5.2.1b) there are two distinct P species, each coordinated by four oxygen atoms, and two distinct Al species, coordinated by six oxygen atoms. Figure 5.2.2 shows ${ }^{27} \mathrm{Al}$ and ${ }^{31} \mathrm{P}$ MAS NMR spectra of $\mathrm{AlPO}_{4}-16$. The ${ }^{31} \mathrm{P}$ spectrum in Figure 5.2.2a contains two resonances separated by $\sim 6 \mathrm{ppm}$. The ${ }^{27} \mathrm{Al}$ MAS spectrum reveals a sharp peak at $\sim 4$ ppm and a second component at $12.5 \mathrm{ppm}$ after removal of the quadrupole broadening (Figure 5.2.2b). A proper spectral assignment of these resonances can only be given by first-principles NMR calculations, as part of the multi-technique approach. The presence of the template and water in the 
pores of the material and the resulting framework flexibility and dynamics make that use of the crystal structure in the NMR calculation, as extracted from the diffraction experiment, is not necessarily the best initial structure to employ. Table 5.2.1 reports the theoretical NMR predictions from a full geometry optimization including the unit cell and from the original diffraction structure.

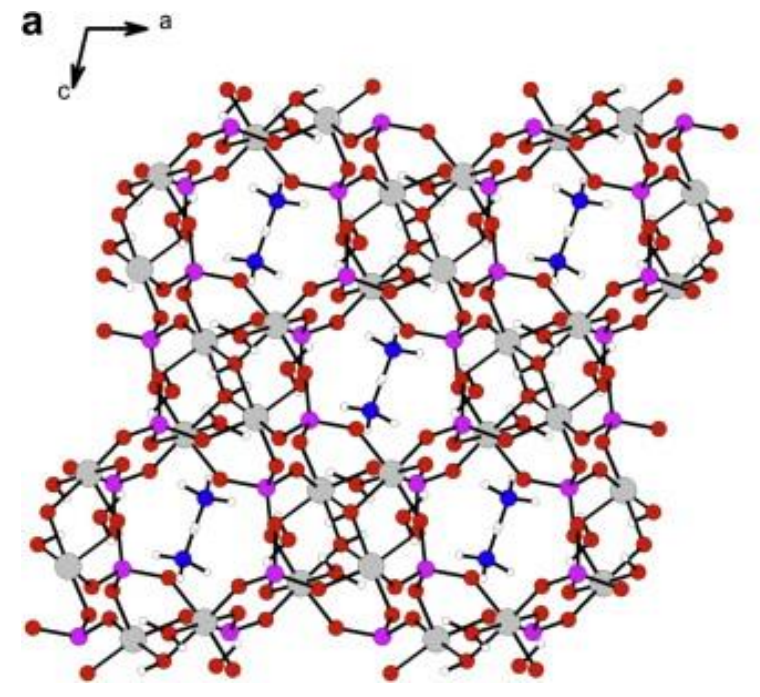

b

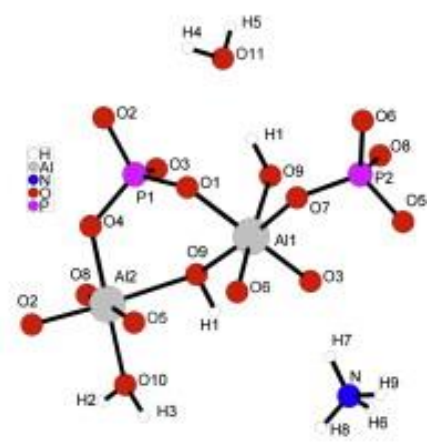

Figure 5.2.1 : (a) Structure of as-synthesized $\mathrm{AlPO}_{4}-15$ with ammonium template (b) Expansion showing the local coordination environments of the $\mathrm{Al}$ and $\mathrm{P}$ species. $\mathrm{P}$ atoms are denoted by pink spheres, $\mathrm{Al}$ by grey spheres, $\mathrm{O}$ by red spheres, $\mathrm{N}$ by blue spheres and $\mathrm{H}$ atoms by white spheres. [reprinted with permission from ref. ${ }^{195}$. Copyright 2009 Elsevier]

Table 5.2.1 : Experimental and calculated ${ }^{31} \mathrm{P}$ and ${ }^{27} \mathrm{Al}$ isotropic chemical shifts $<\delta_{\text {iso }}>$ for assynthesized $\mathrm{AlPO}_{4}-16$. Also taken up are the quadrupolar coupling constant $\left(C_{Q}\right)$ and asymmetry $\left(\eta_{Q}\right)$. Results from ref. ${ }^{195}$.

\begin{tabular}{lccc|ccc|ccc|}
\hline & \multicolumn{3}{c|}{ experiment } & \multicolumn{3}{c|}{ original diffraction structure } & \multicolumn{3}{c}{ full geometry optimization } \\
\cline { 2 - 10 } & $\begin{array}{c}\mathrm{C}_{\mathrm{Q}} \\
(\mathrm{MHz})\end{array}$ & $\eta_{\mathrm{Q}}$ & $\begin{array}{c}<\delta_{\text {iso }}> \\
(\mathrm{ppm})\end{array}$ & $\begin{array}{c}\mathrm{C}_{\mathrm{Q}} \\
(\mathrm{MHz})\end{array}$ & $\eta_{\mathrm{Q}}$ & $\begin{array}{c}<\delta_{\text {iso }}> \\
(\mathrm{ppm})\end{array}$ & $\begin{array}{c}\mathrm{C}_{\mathrm{Q}} \\
(\mathrm{MHz})\end{array}$ & $\eta_{\mathrm{Q}}$ & $\begin{array}{c}<\delta_{\text {iso }}> \\
(\mathrm{ppm})\end{array}$ \\
\hline $\mathrm{Al}(1)$ & -3.1 & 0.80 & $2.5(10)$ & -3.50 & 0.68 & 2.8 & -2.88 & 0.47 & 1.5 \\
$\mathrm{Al}(2)$ & 8.3 & 0.80 & $-6.0(10)$ & 8.25 & 0.94 & -4.6 & 8.29 & 0.86 & -4.9 \\
\hline $\mathrm{P}(1)$ & & & $-14.3(5)$ & & & -12.4 & & & -10.8 \\
$\mathrm{P}(2)$ & & & $-20.5(5)$ & & & -19.1 & & & -17.8 \\
\hline
\end{tabular}

The differences between chemical shifts resulting from the two theoretical calculations are small. The two ${ }^{31} \mathrm{P}$ resonances are separated from each other by $6 \mathrm{ppm}$, in agreement with the experimental spectrum in Figure 5.2.2a. The large difference in quadrupolar coupling $C_{Q}$ between the two $\mathrm{Al}$ species $\mathrm{Al}(1)$ and $\mathrm{Al}(2)$ is well reproduced. This is not trivial as very sensitive to the local environment. Without the support from theory it would be difficult to give the correct interpretation to the measured spectra. 
This test case shows the power of first-principles NMR calculations in interpreting the experimental spectra and to assign peaks to distinct atoms in the framework.

a)

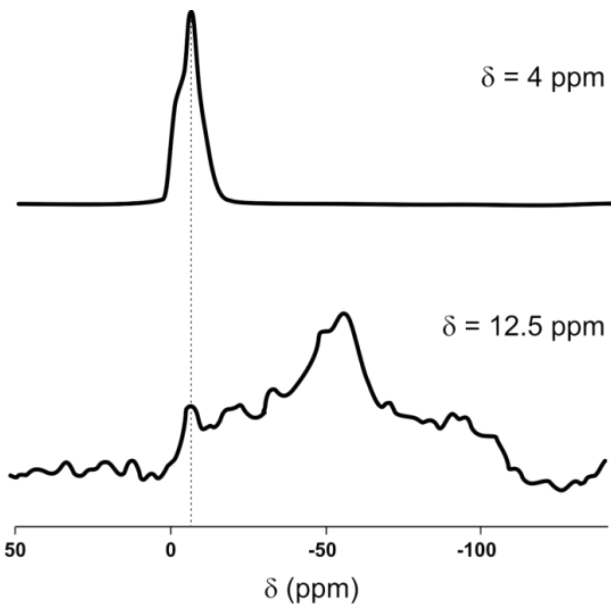

b)

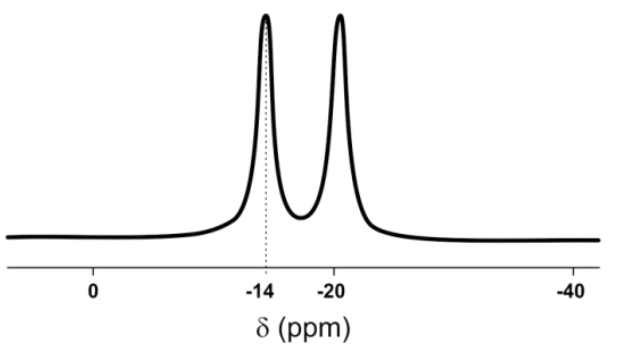

$27 \mathrm{Al}$

$31_{P}$

Figure 5.2.2: ${ }^{27} \mathrm{Al}$ (a) and ${ }^{31} \mathrm{P}$ (b) MAS NMR spectra of as-synthesized $\mathrm{AlPO}_{4}-16$. Results taken from ref. ${ }^{195}$. Adapted with permission from ref. ${ }^{195}$

\section{Structure-spectrum relationships:}

The computation of NMR-parameters with the help of DFT-calculations on very large structures (of a few hundred atoms, such like materials with very large unit cells, disordered supercells, etc... is constrained by huge amounts of required computational time. Very accurate structure-spectrum relations could provide a solution here, but simple linear relations between the isotropic chemical shift and a structure parameter are far from being accurate. Relationships should be refined and in many cases extended to a dependence of two geometrical parameters. Within this respect, the recent work of Dawson et al. ${ }^{196}$ can be regarded as pioneering, demonstrating that a nearly perfect structure spectrum relationship can be constructed in calcined AIPOs between ${ }^{31} \mathrm{P} \sigma_{\text {iso }}$ and two geometrical parameters, characterizing the surface (the average $\mathrm{P}-\mathrm{O}-\mathrm{Al}$ bond angle $\left\langle\theta_{\mathrm{POA}}\right\rangle$ and average $\mathrm{P}-\mathrm{O}$ distance $\left\langle\mathrm{r}_{\mathrm{PO}}\right\rangle$ ). The relationship comprises 159 crystallographically distinct $\mathrm{P}$ species, and is established with the help of first-principles DFT calculations on different structures. The 
advantage of using theoretical rather than experimental data is that in the former there is a unique correspondence between calculated NMR parameters and the structure of the material. In view of the success of this methodology for calcined AIPOs, it could be interesting to investigate how far these principles can be extended and applied to other porous materials related to calcined AIPOs, such like as-made AIPOs, containing SDAs within the pores and charge balancing anions bound to the framework. There exists also no reason why these principles will not hold for aluminosilicate zeolites or any other zeolitic composition. It is unlikely that these simplistic relationships can truly rival firstprinciples calculations, but in very complex systems with framework-bound anions, or other guest species in the pores, for which DFT calculations are very expensive, these structure-spectrum relationships can be of great use to interpret the NMR spectra, and to reveal aspects of the local structure of the material.

These simplistic structure-spectrum relationships have a lot of perspectives when studying dynamic processes in the material, such as the presence of dynamic guest molecules within the pores, with the result that the chemical shifts may vary during time. MD simulations are then very suited if one is able to calculate the NMR parameters at some time steps, which can easily be many tens of thousands. Making use of such simplistic structure-spectrum relationships facilitates the execution of such simulation.

\section{Silicate oligomerization and assistance in templated synthesis of zeolites:}

Molecular modeling is a useful tool to assist in the design of a new structure directing agent (SDA) for the synthesis of zeolites, or to unravel the mechanistic role of the template in the synthesis process. As discussed in section 3.3 the search for a suitable and efficient template is a long-standing issue in the synthesis community. The choice of a template depends on the composition and topology of the envisaged material with the desired chemical and physical properties, and from the plausible candidates preference is given to the species which can be purchased at the lowest price (economical motifs). The experimental approach comprises multinuclear NMR and diffraction studies, but spectra of new materials require a correct assignment of the NMR resonances, and in the exploration phase (different environment of NMR active nuclei results into "unknown" chemical shifts) theoretical support is requisite. We discuss the synthesis of aluminophosphate-based solid STA-2 as an illustrative case. The SDA used for this solid was the dicationic 1,4-bis-Nquinuclidiniumbutane (BQNB) as the best performing among the family of diquinuclidinium cations $\left[\mathrm{C}_{7} \mathrm{H}_{13} \mathrm{~N}-\left(\mathrm{CH}_{2}\right)_{n}-\mathrm{N}_{2} \mathrm{C}_{6} \mathrm{H}_{12}\right]^{2+}$ with linking methylene groups of different lengths ( $\left.n=3-10\right)$ (Scheme 5.2.1 , structure 1). The objective is to replace BQNB by an alternative and cheaper template based on diazabicyclooctane (DABCO) connected by polymethylene chains of different lengths $\left[\mathrm{C}_{6} \mathrm{H}_{12} \mathrm{~N}_{2}-\left(\mathrm{CH}_{2}\right)_{n}-\right.$ $\left.\mathrm{N} 2 \mathrm{C}_{7} \mathrm{H}_{13}\right]^{2+}$ with $\mathrm{n}=3-6$ (Scheme5.2. 1 , structure 2 ) in an attempt to improve the applicability of 
STA-2. ${ }^{197}$ The charge of the DABCO-based templates is found to be divalent. The AIPO ${ }_{4}$ STA-2 material prepared with the help of this template, has been characterized by XRD and multinuclear NMR. The charge balance is achieved by the coordination of hydroxyl ions to framework Al. The obtained $\mathrm{AlPO}_{4}$ STA-2 structure after calcination has been determined with NMR measurements but the full assignment of the ${ }^{27} \mathrm{Al}$ and ${ }^{31} \mathrm{P}$ MAS NMR spectra needed the support of DFT calculations. First, modeling of the new DABCO templates reveals that the most ideal number of methylene groups is four, forming bis-diazabicyclooctane (BDAB). For a larger number of methylene groups the SDA needs to twist to fit into the cage, as clearly seen in Figure 5.2.3. At $n=4$ the torsion energy is found to be a minimum. Secondly, once the most appropriate template has been chosen, the structure of this new $\mathrm{AlPO}_{4}$

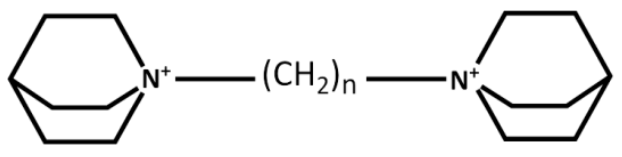

Quinuclidine based

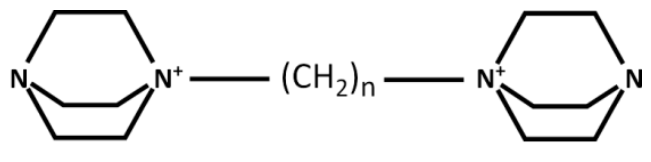

DABCO-based

Scheme 5.2.1 : Quinuclidine and DABCO-based SDAs used in the synthesis of AIPO 4 (STA). Adapted with permission from ref. ${ }^{197}$

$n=3$

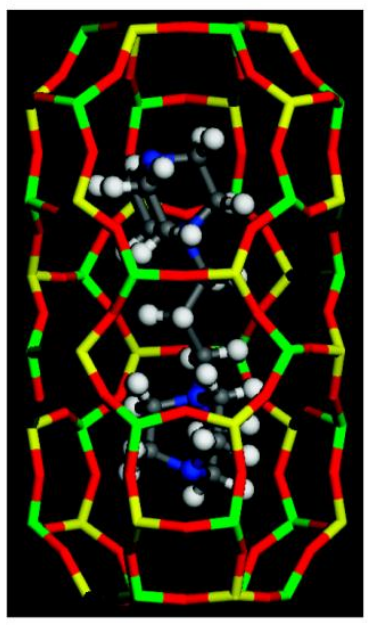

$n=4$

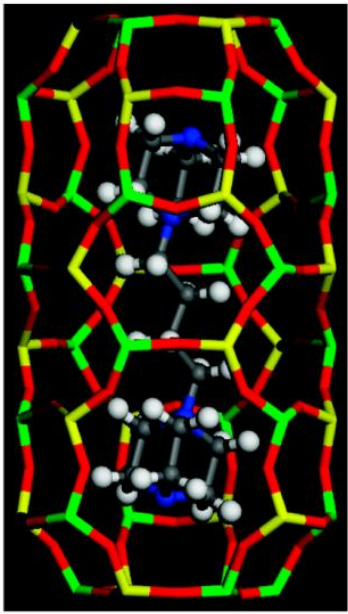

$n=5$

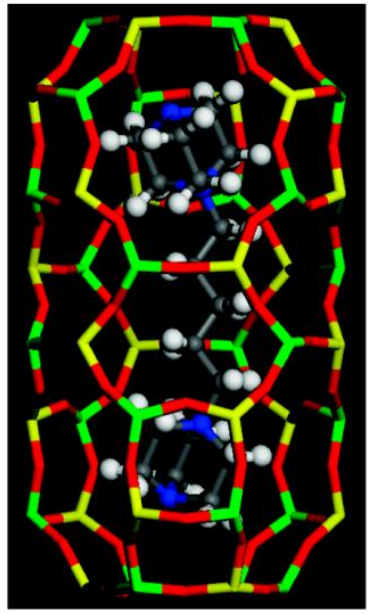

$n=6$

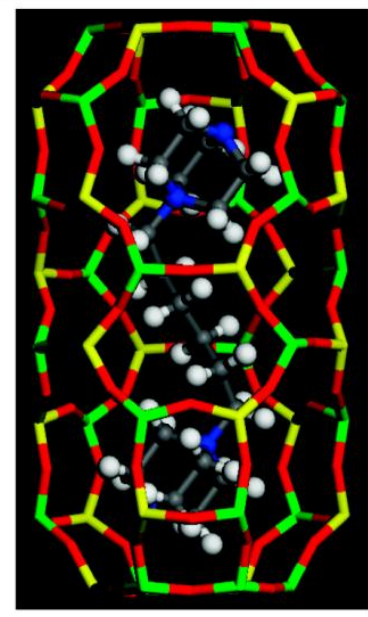

Figure 5.2.3 : Energy-minimized configurations of DABCO-based SDAs with different lengths of linking methylene chains in of $\mathrm{AlPO}_{4}$ (STA). [reprinted with permission from ref. ${ }^{197}$. Copyright 2010 American Chemical Society]

STA-2 solid should be examined using standard experimental techniques including NMR. In an asprepared sample the position of the organic SDA in the pores (as visualized in Figure 5.2.3) is 
analyzed within ${ }^{13} \mathrm{C}$ CP MAS NMR, but a correct interpretation of the resonances can only be done with the assistance of theoretical calculations to estimate the influence of the modified surrounding aluminophosphate framework on the ${ }^{13} \mathrm{C}$ chemical shifts. A similar procedure is followed for the ${ }^{27} \mathrm{Al}$ and ${ }^{31} \mathrm{P}$ MAS NMR spectra. For further details we refer to ref. ${ }^{197}$. It is the combination of experimental and theoretical NMR methods that has led to the conclusion that BDAB is the favored DABCO-based template for STA-2: it acts as a dication and the charge balancing occurs, at least in parts, by the inclusion of hydroxyl groups bridging the $\mathrm{Al}(1)$ and $\mathrm{Al}(2)$ cations. After calcination and removal of the SDAs the structure of the calcined AIPO ${ }_{4}$ STA-2 material, ${ }^{27} \mathrm{Al}$ and ${ }^{31} \mathrm{P}$ MAS NMR experiments are again the most obvious techniques for structure determination. They point to the existence of two crystallographically distinct $\mathrm{Al}$ and P sites in the framework, and that they are all tetrahedrally coordinated, but this assignment was only possible with the support of DFT calculations.

Apart from assistance in the structure determination of SDA directed materials, molecular modeling and first-principles calculation of NMR chemical shifts can also be used to give insight in the formation of elementary framework building units in the early stages of zeolite synthesis, and in particular the initial molecular steps of the acid-catalyzed silica sol-gel process.

The initial steps of silica condensation in silicate oligomerization - of importance in silica-sol-gel chemistry - can also be unraveled by a combined experimental-theoretical approach which leads to the identification of the majority of the formed silicate species. Relevant spectroscopic techniques are here ${ }^{29} \mathrm{Si}$ NMR and UV-Raman. It has been found that oligomerization pathways starting from tetramethylorthosilicate (TMOS) and tetraethylorthosilicate (TEOS) are significantly different. ${ }^{198,199}$ With TEOS rings are formed at the early stages of the oligomerization through cyclo-dimerization; with TMOS this process of ring formation only starts late in the oligomerization scheme. This insight in the different mechanisms in the formation of oligomers is very useful for the design of new silicagel materials, but computational support was here indispensable to give correct interpretation to spectral shifts resulting from chain growth, cyclo-dimerization and branching. Raman spectroscopy is insufficient to unambiguously discriminate the signals of different silicate species and to assign it uniquely to elementary building blocks. ${ }^{29} \mathrm{Si}$ NMR spectroscopy forms here a complementary and indispensable tool to explore the whole process of the oligomerization.

Table 5.2.2 : Experimental and theoretical ${ }^{29} \mathrm{Si}$ NMR chemical shifts and UV-Raman shifts of silicate oligomers departing from TEOS. The $r$-value is a measure of the water:alkoxide molar hydrolysis ratio. Values taken from ref. ${ }^{198}$. 


\begin{tabular}{|c|c|c|c|c|c|}
\hline \multirow[t]{2}{*}{$Q^{i}$ region } & \multirow[t]{2}{*}{ species } & \multicolumn{2}{|c|}{${ }^{29} \mathrm{Si}$ NMR chemical shifts (ppm) } & \multicolumn{2}{|c|}{ Raman shifts $\left(\mathrm{cm}^{-1}\right)$} \\
\hline & & $\exp (r=0.7)$ & theory & $\exp$ & theory \\
\hline $\mathbf{Q}^{0}$ & monomer & -82.50 & $-82.1 ;-82.9$ & 655 & $645-665$ \\
\hline$Q^{1}$ & $\begin{array}{l}\text { dimer } \\
\text { end group }\end{array}$ & $\begin{array}{l}-89.36 \\
-89.53 ;-89.75\end{array}$ & $-86.8 ;-92.7$ & 600 & $585-605$ \\
\hline \multirow[t]{3}{*}{$Q^{2}$ chains } & trimer & -96.72 & & 580 & $560-580$ \\
\hline & tetramer & -96.89 & $-96.7 ; 103.9$ & 565 & $540-560$ \\
\hline & pentamer & -97.10 & & - & $535-555$ \\
\hline \multirow[t]{4}{*}{$Q^{2}$ rings } & 3-ring & -88.4 & $-86.0 ;-88.4$ & - & $580-600$ \\
\hline & 4-ring & -96.69 & & 540 & $535-555$ \\
\hline & 5-ring & -96.94 & $-94.2 ; 100.4$ & - & $530-550$ \\
\hline & 6-ring & -96.10 & & - & $540-560$ \\
\hline$Q^{3}$ & $\begin{array}{l}\text { branched chain } \\
\text { branched ring }\end{array}$ & $\begin{array}{l}-103.3 \\
-102\end{array}$ & $-103.5 ;-106.8$ & & \\
\hline
\end{tabular}

The Raman signatures of the monomer, dimer and linear trimer are readily identified by inspecting experimental and theoretical wavenumbers in Table 5.2.2. The identification of the $565 \mathrm{~cm}^{-1}$ peak is more complex as molecular modeling predicts tetramer vibrations to occur in the region $540-560$ $\mathrm{cm}^{-1}$. Tetramers can occur in the "open" (linear) and "closed" (curled up into closed tetramer) configuration (see Figure 5.2.4). The lower limit of $540 \mathrm{~cm}^{-1}$ stems from the global stretching vibration of the linear tetramer. It converts into a ring breathing vibration as the chain curls up leading to higher wavenumbers. On basis of the spectroscopic signatures relying on ${ }^{29} \mathrm{Si} \mathrm{NMR}$ and molecular modeling a distinction between the Raman signals of the linear and the curled up tetramer and rings with 4-6 Si atoms, could be uniquely made. Once the assignment of each peak in the experimental spectra has been established the whole oligomerization process departing from TEOS can be investigated by in situ ${ }^{29} \mathrm{Si} \mathrm{NMR}$ and UV-Raman spectroscopy. The main condensation reaction pathways were identified as chain extension of dimer through monomer addition, formation of rings via cyclodimerization and branching through attachment of dimer and trimer. Oligomers barely grew larger than $6-9 \mathrm{Si}$ atoms, chains no longer than pentamer and ring sizes limited to 6-rings. The same procedure has been exploited in a similar study on the conversion of TMOS into silica gel network under condition of acid catalysis. The insight gathered into the chemical nature of precursor oligomers may be of great help in the tuning of the synthesis of amorphous microporous silica materials. 


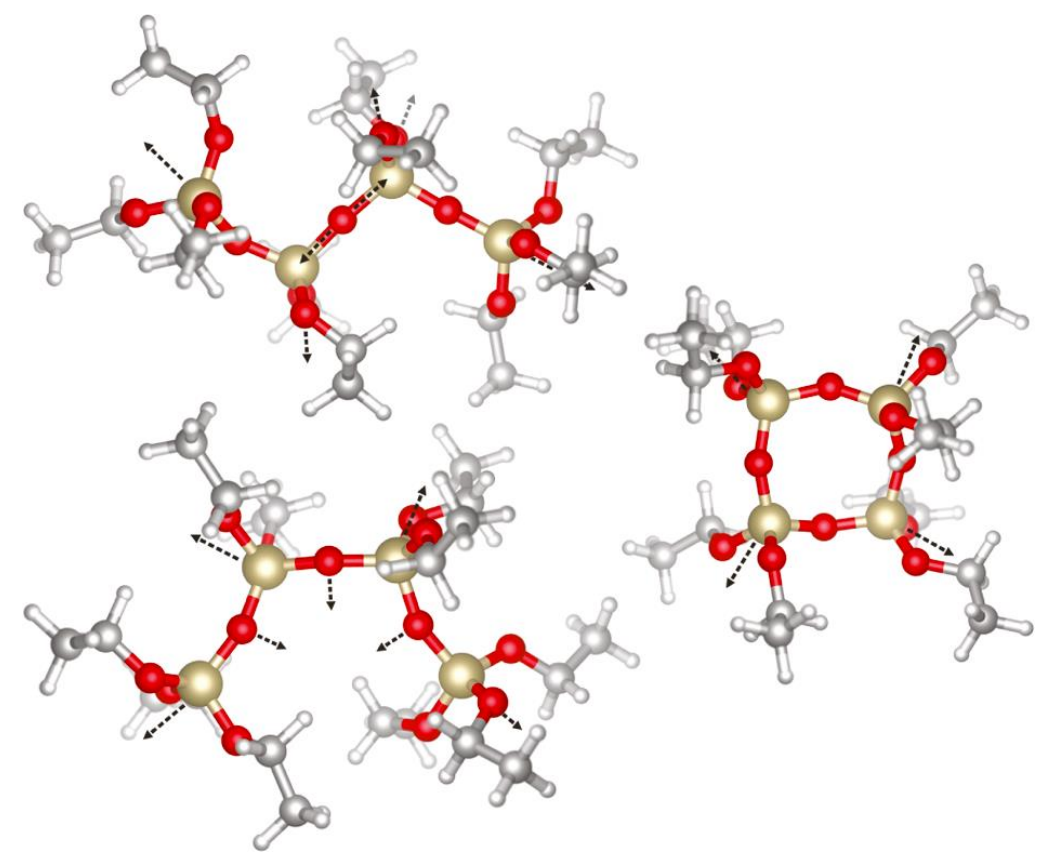

Figure 5.2.4 : Visualization of the tetramer species (open and closed configuration and 4-rings). Reprinted with permission of ref. ${ }^{198}$.

\section{Measuring zeolitic acidity using NMR technique:}

Apart from structure characterization NMR technique can indirectly be applied to measure the acid strength of acidic zeolites. As an illustration, solid-state ${ }^{31}$ P MAS NMR of adsorbed trimethylphosphine oxide (TMPO) is a unique and practical technique for acidity characterization of solid acid catalysts. ${ }^{200}$ The technique is based on a linear correlation between the ${ }^{31} \mathrm{P}$ chemical shifts of TMPO and the proton affinity and hence the BAS. A higher observed $\delta^{31} \mathrm{P}$ value implies a stronger acidic strength, while a value of $88 \mathrm{ppm}$ corresponds with a strong Brønsted acidity. Theoretically the procedure consists in studying the adsorption of TMPO as probe molecule near the acid site with a suitable DFT+D methodology and to calculate the isotropic $\delta^{31} \mathrm{P}$ value, which is a measure of the acid strength.

\section{Future prospects - positioning of hetero atoms in zeolites:}

The application field of solid-state NMR is still growing and most of the progress is due to the increasing accuracy of the measurements. With MAS NMR a very powerful technique has been developed which is currently applied for structure determination of zeolite materials. It is also highly suited to assist in positioning hetero atoms in rings. The presence of hetero atoms in the framework may change the catalytic properties of zeolites, but may also affect the topology obtained during synthesis. The localization of especially $\mathrm{Al}$ in siliceous zeolites is of great importance to understand 
the catalytic activity, but neither X-ray nor neutron diffraction or spectroscopy can distinguish Al from Si. Within this aspect the recent work of Cadars et al. ${ }^{201}$ is a breakthrough in the sense that they propose state-of-the-art NMR methods that allow the identification and quantification of the signatures of otherwise undetectable non-crystalline impurities, thus largely complementing the traditional elemental analyses. The authors succeeded in determining structural ordering in aluminosilicate clays. Experimental information on the molecular structure of clay minerals is particularly difficult to gain, because of small crystallite sizes, morphological and structural heterogeneity, variable composition and complexity of their layer structure. High resolution ${ }^{27} \mathrm{Al}$ and ${ }^{29} \mathrm{Si} \mathrm{NMR} \mathrm{spectroscopy} \mathrm{is} \mathrm{required} \mathrm{to} \mathrm{establish} \mathrm{the} \mathrm{different} \mathrm{chemical} \mathrm{environments} \mathrm{of} \mathrm{Si} \mathrm{atoms} \mathrm{in}$ clay minerals. It is the unique combination of ${ }^{29} \mathrm{Si},{ }^{27} \mathrm{Al},{ }^{25} \mathrm{Mg}$, and ${ }^{1} \mathrm{H}$ NMR with DFT calculations that makes feasible the unraveling of the complicated intralayer atomic arrangements. The different NMR signals are rationalized on the basis of various local environments.

The solid-state NMR technique can be further extended to study other NMR-active elements coming into play in new zeolites, like germanium containing Si-zeolites, as already discussed in section 4.2. The use of $\mathrm{Ge}$ as hetero-element in the synthesis process is known to generate zeolite frameworks with extra-large pores. ${ }^{116,117}$ These large ring sizes are mainly due to the highly flexible O-Ge-O angles and bond lengths, which are also able to stabilize small cage structures like double four- and three-rings (D4R and D3R) at the adjacent large pore. ${ }^{117}$ Figure 5.2 .5 shows the energy profile for the T-O-T angle, with T=Si or Ge, relative to the relaxed angle. The profile for the Si-O-Si angle has a minimum around $150^{\circ}$ and there is only a small energy difference for larger values $\left(<5 \mathrm{~kJ} \mathrm{~mol}^{-1}\right.$ for $180^{\circ}$ ). For Si-O-Si angles below $135^{\circ}$, the energy penalty is much higher but this is exactly the region where the Ge-O-Ge angle attains a minimum energy. Such acute angles can be found in D4R units, explaining why $\mathrm{Ge}$ is preferably incorporated in these units.

The fluoride synthesis route is the most commonly used method to fabricate Si/Ge zeolites. The role of the $\mathrm{F}^{-}$anion in the synthesis process is not exactly known, but it is generally accepted that it resides near a Ge atom in the Ge-containing D4R units and ${ }^{19} \mathrm{~F}$ NMR chemical shifts should in principle be able to rationalize the different signals arising from the different chemical composition of the D4Rs. Germanium is able to form a covalent bond with fluoride, yielding a five-folded coordinated germanium in the D4R units that make up the AST structure. Silicon cannot form a covalent bond with $\mathrm{F}^{-}$and therefore lacks stability in the D4R. The formation of D4R units in Si/Ge zeolites in presence of fluoride anions is more favorable for $\mathrm{Ge}$ than for $\mathrm{Si}$, because of the larger stability of the covalent Ge-F bond with respect to the ionic Si...F interaction. ${ }^{118} \mathrm{~A}$ first computational study of ${ }^{19} \mathrm{~F}$ NMR spectra has been performed by Pulido et al. ${ }^{202}$, but these 


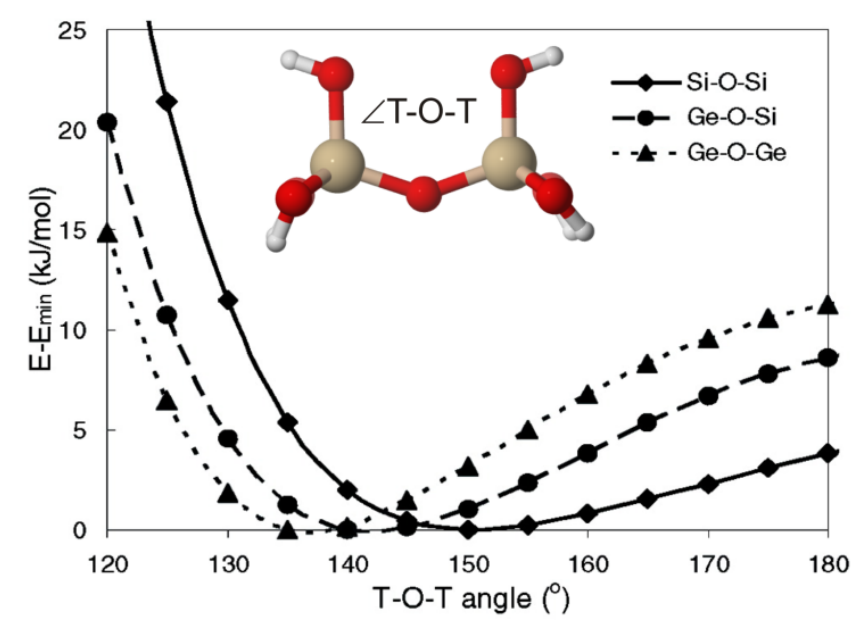

Figure 5.2.5: Energy profile for the T-O-T angle with $\mathrm{T}=\mathrm{Si}$ or $\mathrm{Ge}$, relative to the relaxed angle.

Adapted with permission from ref. ${ }^{119}$.

authors used cluster models to model the D4R units. Very recently fluoride ions have been incorporated post-synthetically in the structure of as-made germanium-containing zeolites, without modification of the framework composition (Si/Ge ratio) and of the crystallinity. ${ }^{203}{ }^{19} \mathrm{~F}$ NMR has been used to probe the location and distribution of Ge atoms in the framework. In ITQ-13 and ITQ17 the incorporated $\mathrm{F}^{-}$anions are located in the same structural units as those occupied when the material is prepared in the presence of fluoride. In the case of ITQ-22 and ITQ-24, the fluoride goes mainly in D4R units. Zeolites prepared in the absence of fluoride in the synthesis contain more Ge atoms in the $\mathrm{D} 4 \mathrm{R}$ units with formation of [4Si,4Ge] units. First principles NMR studies - preferentially with periodic codes - play here a dominant role in the rationalization of the observed ${ }^{19} \mathrm{~F} N \mathrm{NR}$ signals. However, despite the success of ${ }^{19} \mathrm{~F}$ NMR in exploring the composition of the D4R units, a more direct method to determine the framework structure would be a refinement of the chemical shifts in ${ }^{29} \mathrm{Si} \mathrm{NMR} \mathrm{spectra,} \mathrm{where} \mathrm{substitution} \mathrm{of} \mathrm{framework} \mathrm{Si} \mathrm{by} \mathrm{Ge} \mathrm{modifies} \mathrm{the} \mathrm{spectrum,} \mathrm{but} \mathrm{this}$ direct method is not yet developed to clearly resolve the crystallographic ordering in germanozeolites. Such a methodology would be very useful as Ge containing zeolites are also synthesized in the absence of fluoride, and structure characterization should be made possible without using extra-framework species like ${ }^{19} \mathrm{~F}$. This new strategy for structure characterization is highly welcome for structure transformations, which recently caught considerable attention. ${ }^{111,112}$ Verheyen et al. ${ }^{111}$ observed that D4Rs often act as a common building unit to expand the framework and to assume the structural role of linking layers. Via the so-called inverse sigma transformation systematic removal of T-atom layers and framework reconnection new topologies are obtained. Germanium-containing four-ring units are optimally suitable for this transformation. Figure 4.2.1 
depicts how germinate 4-rings are dislodged from an IM-12 zeolite (UTL topology with extra-large pores) by acid leaching, allowing a framework contraction whereby the Ge-4R units are shifted into the channels of Ge-COK-14. Further leaching fully eliminates the Ge-atoms, resulting into a Ge-free interrupted framework (-COK-14), that can be fully closed upon calcination (COK-14).

${ }^{29} \mathrm{Si}$ MAS NMR measurements were recorded at different stages during the phase transformation and a thorough analysis of the signals indicates that the intense $Q^{4}-n G e$ signal of IM-12 was much weaker in -COK-14, confirming the extraction of germanium (Figure 5.2.6).
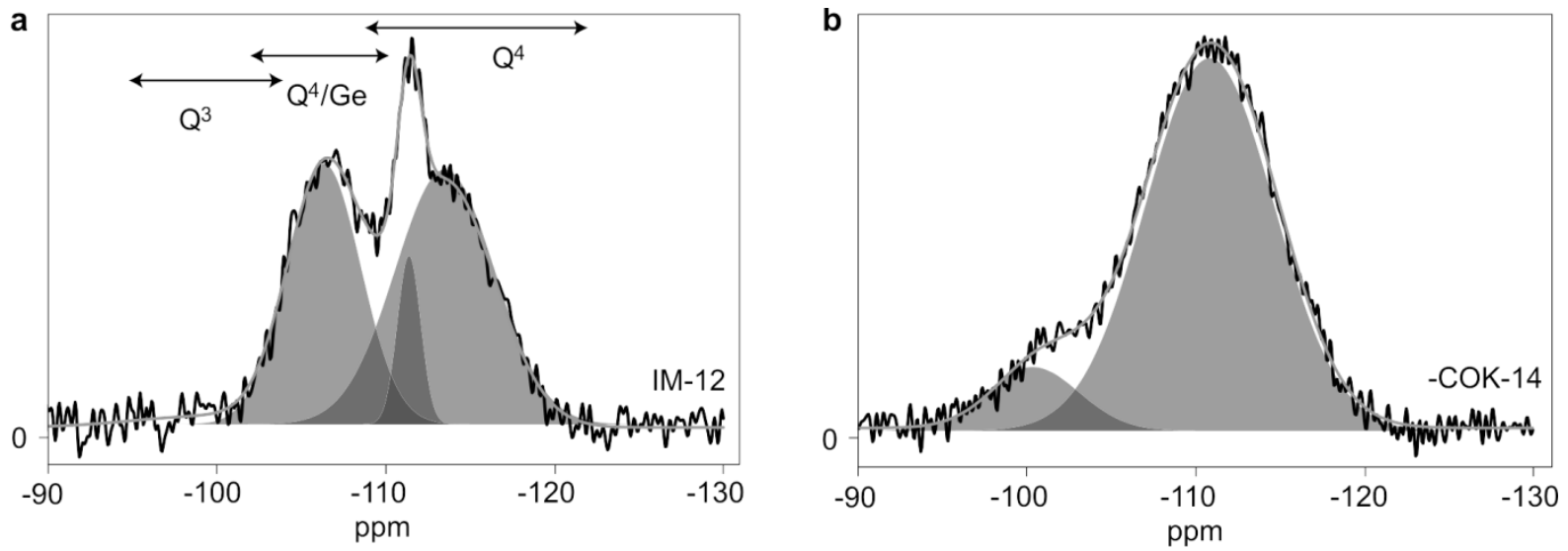

Figure 5.2.6 : Comparison of ${ }^{29} \mathrm{Si}$ NMR spectra in $\mathrm{IM}-12$ and-COK14. Reprinted with permission from ref. ${ }^{111}$

The correct assignment of the signals requires the support of state-of-the-art theoretical calculations. Periodic DFT calculations are also very useful to compare the optimized geometrical structures with those predicted by Rietveld refinement. In addition they give insight on the stability of the different structures. However, the transformation of the interrupted framework of -COK-14 into a fully connected structure COK-14 has not yet been modelled by theory and remains a high challenge to perform. This issue has gained in importance by the recent experimental observation that the chemical and physical transformation from -COK-14 to the closed COK-14 is a reversible process. ${ }^{112}$ Condensation of the interrupted -COK-14 by heating at $923 \mathrm{~K}$ leads to release of water molecules and formation of the highly rigid and fully connected COK-14 framework. The latter is reopened again in the presence of warm water. This transformation is clearly entropy driven, and constitutes a challenging issue for theoreticians to explain the mechanism within advanced MD simulations that can tackle the breaking and forming of bonds. 


\subsection{Optical spectroscopy}

In addition to the aforementioned techniques focusing on the electronic ground state of the involved systems, there is also an urgent need for studying excited state properties in zeolite chemistry. Advanced experimental techniques involving optical light are nowadays applied to study catalyst materials at work, i.e. under operando conditions. ${ }^{132}$ UV/Vis and/or fluorescence spectroscopy can also provide information about the single-molecule detection in zeolite-catalyzed reactions. ${ }^{204}$. Simulation of such excited-state properties of relatively large systems poses new challenges to the available implementations. Computation of optical spectra is becoming more and more possible, thanks to developments in theoretical algorithms, most importantly in timedependent density functional theory (TD-DFT). ${ }^{205,206}$ To date, TD-DFT is feasible for systems with up to 400 atoms and an accuracy of $0.5-0.4 \mathrm{eV}$ for excitation energies can be reached. ${ }^{207}$ Important evolutions is this field are mainly linked to two aspects: (1) use of MD simulations for sampling the potential energy surface ${ }^{208,209}$, (2) inclusion of temperature effects. ${ }^{210-213}$ Focusing on the first aspect, it is accepted that if the spectroscopic transition is fast compared to the time-scale of the dynamical phenomenon, averaging over different configurations - generated during a dynamical simulation - is the best technique to obtain theoretical results which can complement measured data. ${ }^{208,209}$ The resulting averages are an estimate of the electronic transition energy and linewidth.

In the field of zeolite chemistry, some of us applied this technique to compute excitation properties of aromatic carbonaceous species representing reaction intermediates formed during the methanolto-olefins process (see further sections 6.2 and 6.4). ${ }^{214}$ An extended set of both neutral and charged aromatics varying in their size and degree of methylation was considered and dynamic averages of vertical excitation energies were computed. It was found that the B3LYP functional performs best for the neutral compounds, whereas the CAM-B3LYP functional is needed for the cationic species. The MD-based approach, accounting for the flexibility of the carbonaceous compounds, leads to a redshift of approximately $30 \mathrm{~nm}$ and is essential to provide good agreement with available experimental data. A theoretical assignment scale was constructed and served as input for structure characterization of experimentally determined in-situ UV/Vis absorption bands (see Figure 5.3.1). ${ }^{215}$ 
a)

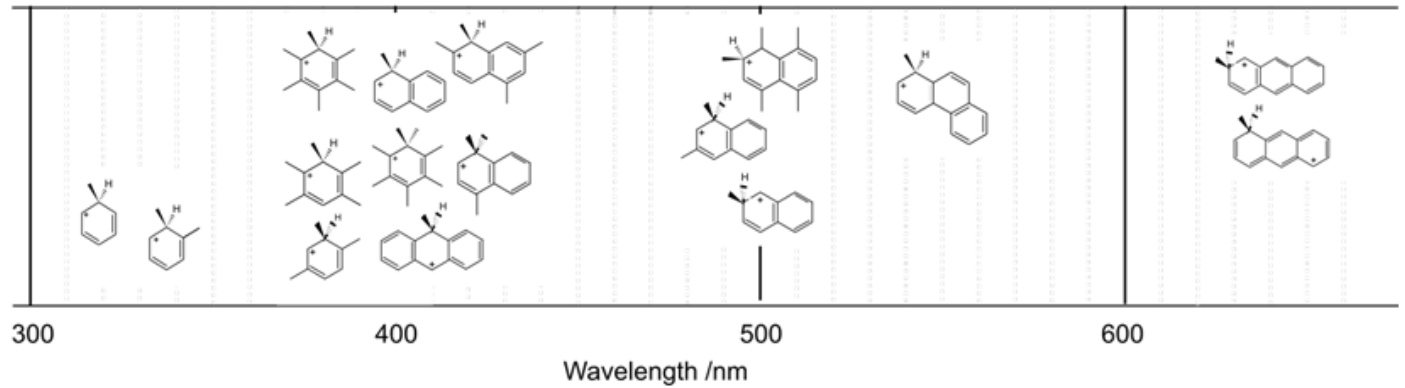

b)

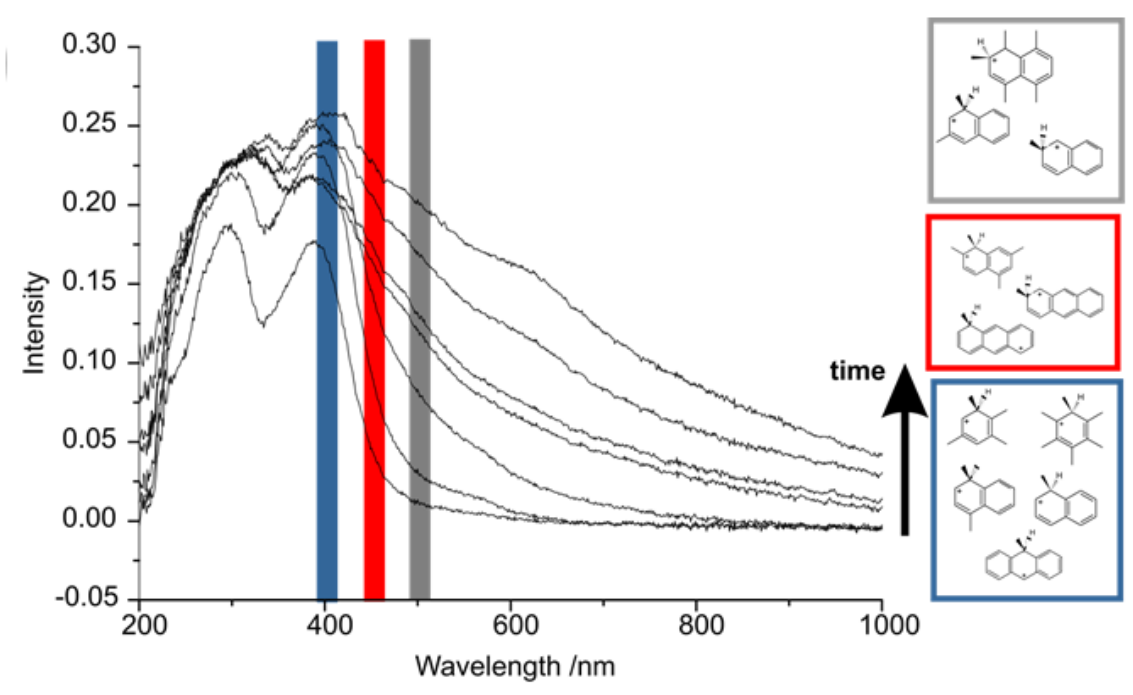

Figure 5.3.1 : Assignment scale constructed from MD-averaged TD-DFT computations (a). Use of the assignment scale to assign bands in in-situ UV/Vis absorption spectra to structurally different aromatic HP species. (b). Adapted with permission from ref. ${ }^{215}$.

Modeling excitation properties involving porous zeolitic materials often remains challenging, mainly due to the large size of the involved systems. Most often, the zeolite environment is taken into account either via small clusters or via an embedding procedure. In this light, potassium-rich zeolite L (LTL framework topology) is one of the most investigated zeolite materials. Its 12-ring onedimensional channels (free diameter of $7.1 \AA ̊$ ) allow incorporating a large variety of guest systems. Calzaferri and collaborators have extensively examined zeolite $L$ filled with dye molecules in view of their use as host-guest antenna materials. ${ }^{216}$ The narrow channels can prevent sufficiently bulky molecules from passing each other, allowing the realization of well-defined domains inside the channel system. First-principles simulations were applied to simulate the absorption bands of fluorenone in zeolite L. Initial work provided the computed TD-DFT excitation spectra of relatively small molecular models extracted from the minimum energy structures of the dye-zeolite system, containing the fluorenone dye and the nearest atoms of the zeolite $L$ environment. ${ }^{217}$ From their 
vertical excitations using the hybrid B3LYP level of theory, the authors concluded that the experimentally observed bathochromic shift results from close contacts between the dye carbonyl group and the extra framework potassium ions. This interaction primarily stabilizes the LUMO of the fluorenone dye. In a next step, the dye was embedded in a large zeolite cluster containing $432 \mathrm{~T}$ sites (Figure 5.3.2). ${ }^{218}$ The Frozen-Density Embedding Theory (FDET) was applied to examine both the induced shifts and shape broadening due to the dye-zeolite interactions. This FDET method was previously shown to lead to accurate environment-induced shifts in spectral positions in case of situations where the orbital overlap between the chromophore and its environment is relatively small. ${ }^{219}$ Both the dry and hydrated zeolites were modeled; the latter includes four extra water molecules. The structural conformations of the dye-zeolite complex were taken into account via First-Principles MD (FPMD) simulations and average absorption properties were obtained after performing a Linear Response -TDDFT calculation on a large series of configurations. Importantly, the calculated excitation energies were empirically corrected, using a constant shift of $0.8 \mathrm{eV}$, correcting for the inaccuracies in the calculated excitation energy of gas phase fluorenone and for the vertical approximation. From Figure 5.3.2 b it can be seen that the simulated absorption spectrum reproduces all the main features of the experimental one. Analysis of the simulations reveals that the width results from fluctuations of the geometry of the whole system, whereas the shape is almost unaffected. The influence of addition of water molecules on the absorption properties was also found negligible for the investigated guest-host system.
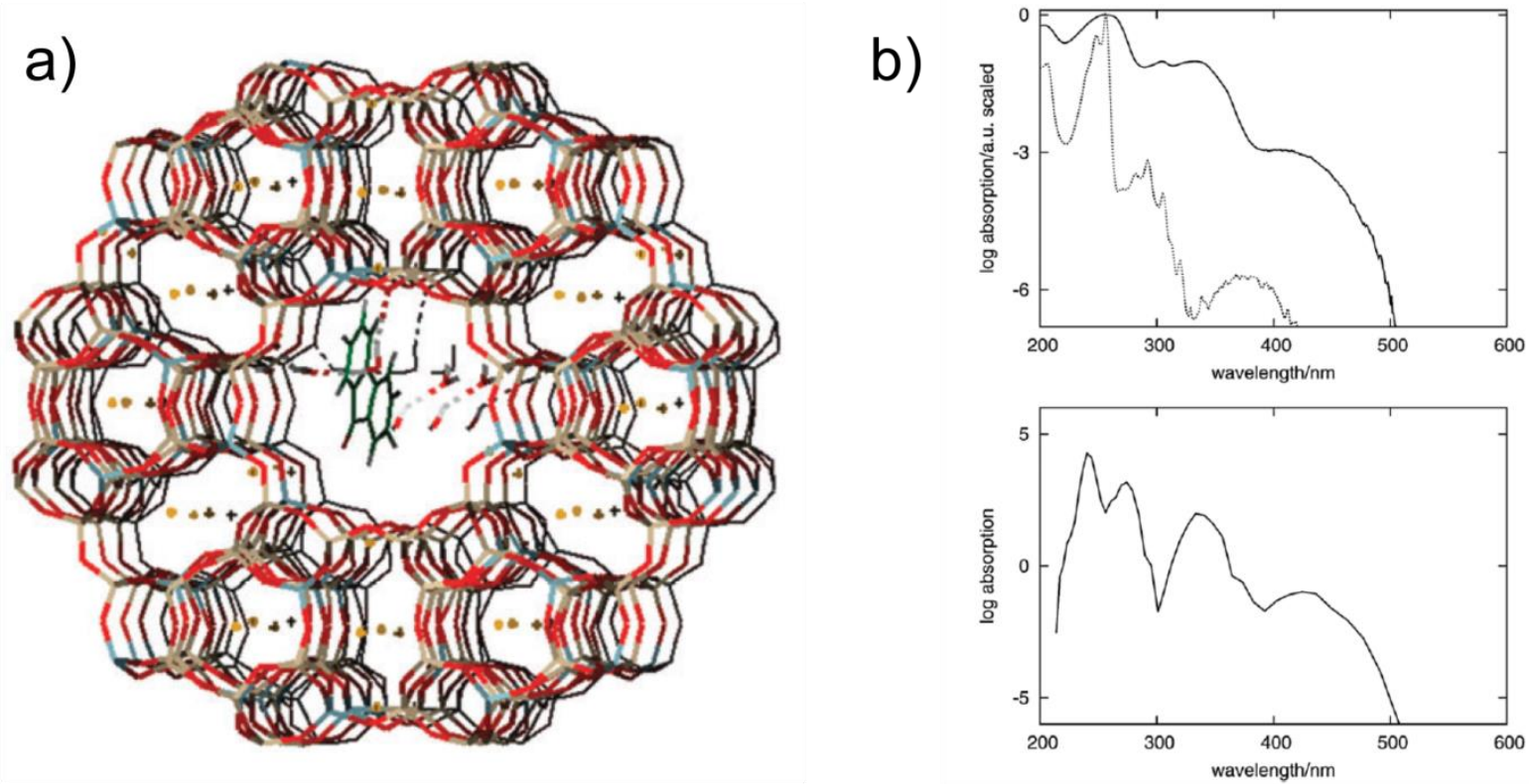

Figure 5.3.2 : a) Cluster used for calculating excitation energies of fluorenone in the hydrated zeolite.

b) Experimental (top) and simulated (bottom) spectrum. The full curve represents the dye in the 
hydrated zeolite, whereas the dashed line represents the dye in cyclohexane solution. Adapted with permission from ref. ${ }^{218}$.

Noteworthy, analysis of the generated First Principle MD trajectories provides valuable information about the organization of the dye molecules in the zeolite channels. ${ }^{218,} 220$

In a similar study on host-guest interactions, the optical properties of 2 coumarin derivatives adsorbed in the mesoporous MCM-11 host has been investigated by Pedone et al.. ${ }^{221}$ In this work, vibrationally resolved electronic spectra including temperature effects were computed on a dye/silica assembly containing more than 120 atoms. Interactions between the dye and the silicate were investigated at the QM level using a slab model for the silicate material. The vibrational resolution of the calculations turned out to be necessary to reproduce the overall band shape of the experimental spectra. This study demonstrates the possibility to calculate highly accurate electronic spectra including interactions with a silica material and including temperature effects and breakthroughs are expected in this field.

\section{Applications using zeolites: selective case studies}

Zeolites are widely used in a broad range of applications as was emphasized in Section 2 . In this section we give a flavor of the added value of modeling within this broad area on basis of some selected case studies. Section 6.1 discusses the case of $\mathrm{CO}_{2}$ capture which is positioned in the application field of gas separation. Indeed carbon capture \& sequestration (CCS) is one of the most promising technologies to reduce $\mathrm{CO}_{2}$ emissions from large power plants on the short term. Adsorption by nanoporous materials has been identified as an efficient process to separate $\mathrm{CO}_{2}$ from $N_{2}$. In view of this, zeolites have received considerable attention from a modeling point of view, to screen zeolites according to their CCS performance. We will highlight some of the recent contributions to show the strengths of current theoretical approaches.

Sections 6.2 and 6.3 discuss several aspects related to the omnipresence of zeolites within catalysis today and the vital role of modeling to obtain molecular control of a given process. Section 6.2 discusses the conversion of hydrocarbons using zeolites with Brønsted acidic functions. Zeolites are indeed the workhorses of current large scale petrochemical industry. We specifically focus on the process of catalytic cracking of long chain hydrocarbons and the conversion of methanol to hydrocarbons. A broad variety of aspects will be discussed such as the adsorption, diffusion of 
reacting agents and the construction of complex catalytic cycles. The section will clearly reveal the importance of spectroscopy to characterize important intermediates.

Section 6.3 shows the importance of advanced molecular modeling to unravel complex catalytic cycles for selective oxidations using zeotype systems which have been doped with transition metals. Furthermore we discuss in section 6.3.3 the case of metal-exchanged small pore zeolites which are used in the ammonia-assisted selective catalytic reduction of NOx. In this section we particularly emphasize the role of theoretical modeling and computational spectroscopy to reveal the position of $\mathrm{Cu}$ in chabazite type zeolites.

Section 6.4 discusses modeling techniques for the description of diffusion and adsorption within zeolites. A proper understanding of diffusion is prerequisite for catalysis and separation technologies.

\section{1. $\mathrm{CO}_{2}$ capture}

Due to their high $\mathrm{CO}_{2} / \mathrm{N}_{2}$ selectivities, zeolites have been proposed as potential candidates for the separation process in Carbon Capture and Storage (CCS). ${ }^{222-225} \mathrm{CO}_{2}$ adsorbs selectively in the pores of the zeolites and the material can afterwards be regenerated by heating (TSA, temperature swing adsorption) or by applying a vacuum (PSA, pressure swing adsorption). This regeneration imposes a substantial energy requirement for CCS, the main bottleneck for its industrial application. Computational tools can help to understand the interactions between $\mathrm{CO}_{2}$ and the nanoporous material, as well as identify where improvements are possible and what the most promising materials are.

To compare zeolites among each other or against other classes of nanoporous materials ${ }^{226}$, Bae and Snurr proposed 5 metrics to evaluate the CCS performance: $\mathrm{CO}_{2}$ uptake, $\mathrm{CO}_{2} / \mathrm{N}_{2}$ selectivity, $\mathrm{CO}_{2}$ working capacity, regenerability of the material and a sorbent selection parameter. ${ }^{227,}{ }^{228}$ Comparing the relative importance of 5 competing parameters for a large set of materials however is not trivial. Therefore, Smit and coworkers suggested one metric instead, the 'parasitic energy', i.e. the energy parasitized by the CCS process. ${ }^{229}$ This metric is highly relevant to the industry, as it straightforwardly determines the energy demand for CCS and allows to pinpoint the material that can achieve CCS with the lowest energy requirement. Moreover, it was recently demonstrated that there exist good correlations between the parasitic energy and criteria proposed by Bae and Snurr. 230

Figure 6.1.1 shows the parasitic energy as a function of the $\mathrm{CO}_{2}$ Henry coefficient for all structures in the IZA database, with varying Si/Al ratios. The negative charges introduced by $\mathrm{Al}$ in the framework are countered with sodium cations. The parameters of the force field are taken from Calero and co- 
workers and have proved to be successful in reproducing experimental $\mathrm{CO}_{2}$ and $\mathrm{N}_{2}$ isotherms. The large number of data points allow the determination of an envelope curve (the black line in Figure 6.1.1). This type of materials screening is thus a convenient tool by which to gauge the minimum parasitic energy for zeolites and to identify the most interesting classes of materials (most of the optimal performing materials have Al in their framework) or interesting regions (materials with Henry coefficients between $10^{-4}$ and $10^{-3}$ have optimal performance). It would be difficult if not impossible to obtain this information from experiments alone, as larger data sets are needed to uncover these kinds of trends.

To calculate the Henry coefficient of a given material, Grand Canonical Monte Carlo (GCMC) simulations at infinite dilution are performed. The so-called Widom particle insertion method randomly inserts a guest molecule inside the framework and calculates the Boltzmann factor for this configuration. ${ }^{231}$ From the statistics, the adsorption enthalpy and Henry coefficient can be determined. Kim et al. proposed an algorithm that uses an energy grid to store the interactions between the framework atoms and the gas molecules. ${ }^{232}$ This approach speeds up the subsequent MC simulation significantly. The building of the grid requires most of the computational time, but when the grid is stored in the permanent memory of a GPU (Graphics Processing Unit), and the MC simulations are also performed on that GPU, the simulation can be up to 50 times faster than on conventional CPU's (Central Processing Unit). ${ }^{233}$ This recent improvement in both algorithm and hardware allows the screening of much larger databases, although the code is less flexible than more established CPU codes.

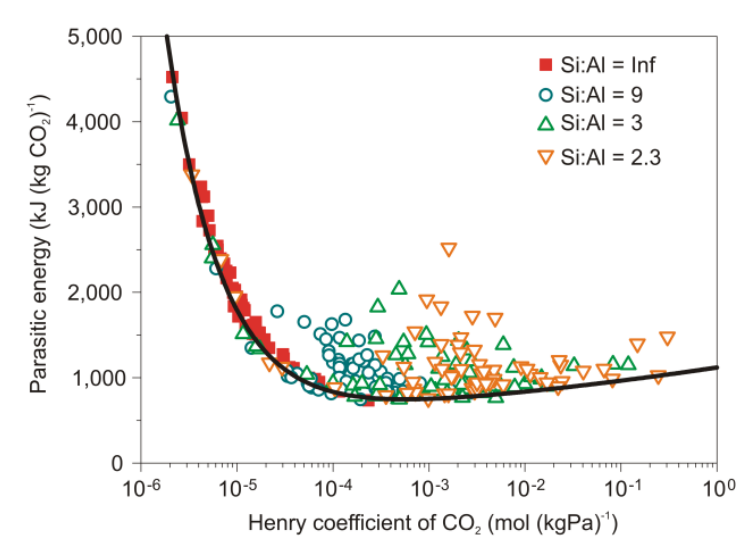

Figure 6.1.1 : Parasitic energy as a function of the $\mathrm{CO}_{2}$ Henry Coefficient, for zeolites with different $\mathrm{Si} /$ Al ratios. Reprinted with permission of ref. ${ }^{229}$

The strength of high-throughput computer screenings lies in their capacity to screen a large area of the material space. One of the possibilities is the identification of the most promising materials for 
one or more desired properties. Another opportunity is to determine the limits of the material properties. As an example, a screening of thousands of porous materials from different families showed that the performance targets for methane storage set by funding agencies are unrealistic. This screening study was able to assess a more realistic limit, determine the material family that has the best properties as well as identify the performance the best materials up to date. ${ }^{234}$ This principle could also be used in the field of CCS.

In Figure 6.1.1., the parasitic energy is plotted as a function of the Henry Coefficient, determined by (computationally expensive) Widom particle insertions. It could therefore be interesting to estimate the performance of a material based on a structural metric, that is computationally easy to determine. ${ }^{235}$ In Figure 6.1.2, the $\mathrm{CO}_{2}$ uptake is represented as a function of the diameter of the largest included sphere, with the highest $\mathrm{CO}_{2}$ uptake for cavities around $10 \AA .{ }^{100}$ Codes like Zeo++ 236-239 and ZEOMICS ${ }^{15,16,18}$ quickly provide geometrical features of nanoporous materials, such as the diameter of the largest included sphere, channel characterization, available pore volume, surface area, pore limiting diameter and pore size distribution.

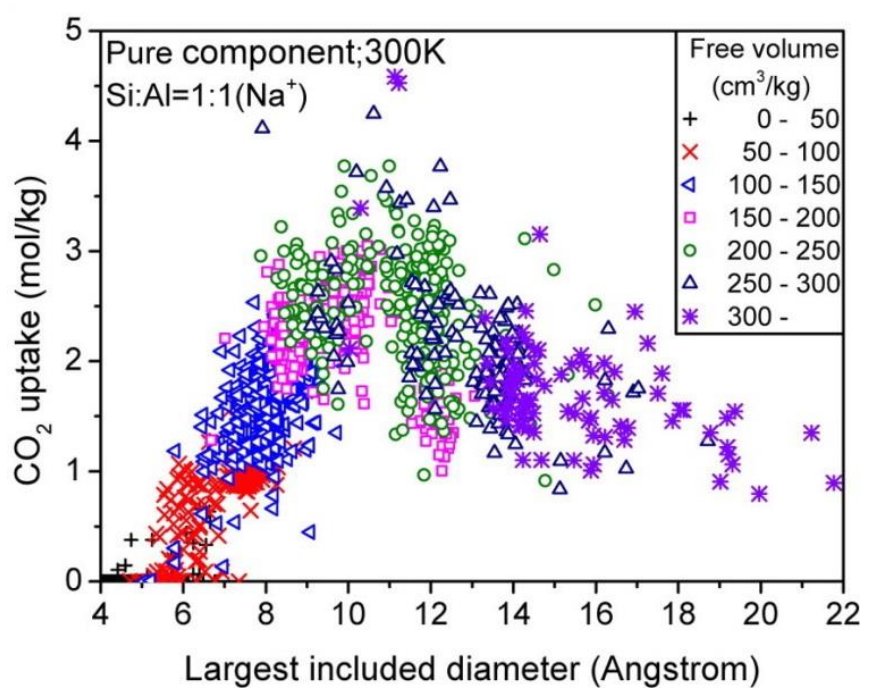

Figure 6.1.2 : $\mathrm{CO} 2$ uptake as a function of the diameter of the largest included sphere. Reprinted with permission of ref. ${ }^{100}$.

Sarkisov and Kim give an excellent overview of recent developments in these structure characterization codes. ${ }^{240}$ Further advances are however necessary to ensure computational efficiency in large-scale screening of geometric identities. Although the diameter of the largest included sphere is a very simple metric, it does not provide all information about the topology of the pores and channel network. A more sophisticated characterization of the effect of topology on adsorption behavior can be obtained using the concept of natural tiling. ${ }^{241,242}$ 
As described in section 4.1 the tiles can be thought of as building blocks that make up the zeolite framework. These units often correspond to the cavities and cages in the framework they describe and their faces represent the structural windows that are also present. Understanding the interaction of gas molecules with the individual tiles and selecting the tiles that have the best interaction with $\mathrm{CO}_{2}$ molecules can therefore help to identify the most favorable frameworks for $\mathrm{CO}_{2}$ adsorption. Fischer et al. ${ }^{241}$ considered 18 different zeolite topologies for $\mathrm{CO}_{2} / \mathrm{N}_{2}$ separation and found that the best performing materials were those with double-crankshaft chains and/or double eight-ring units as components of their framework structure. In a later paper ${ }^{242}$, the work was extended to identify natural tiles (and hence the zeolites which contain them) possessing the most energetically favorable $\mathrm{CO}_{2}$ sites. Three criteria were proposed: (1) the tile should have eight-ring faces, (2) none of the tile's faces should be larger than an eight-ring and (3) at least two of the tile's faces should be four-rings which share a common edge. The tile with the strongest affinity for $\mathrm{CO}_{2}$ was found to be the single tile of the GIS framework, corresponding to the gismondine cage (Figure 6.1.3).
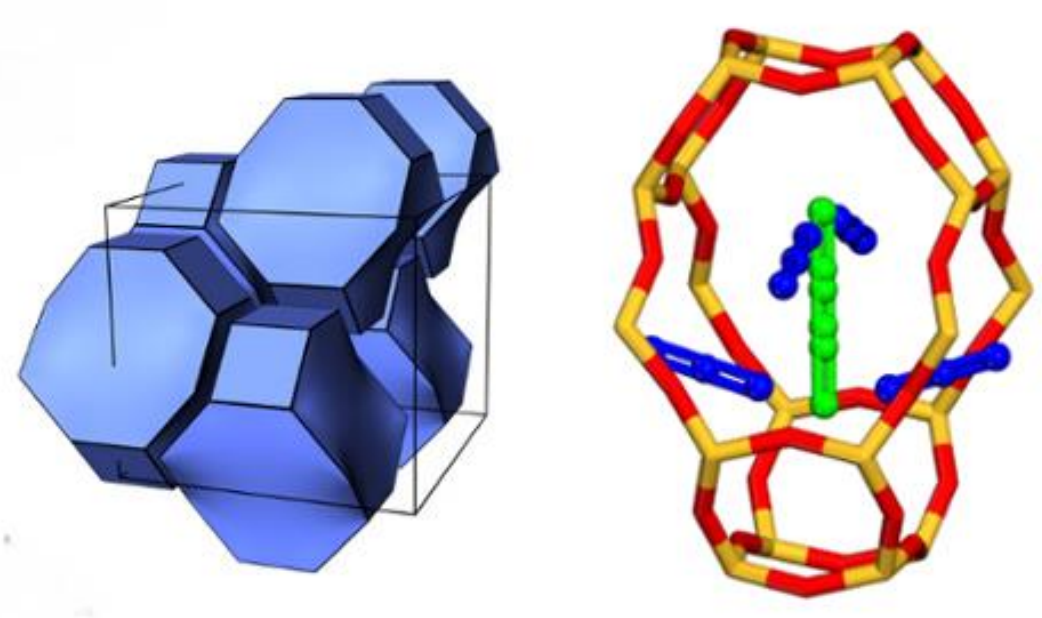

Figure 6.1.3 : Left - Tiling of the GIS (Gismondine) Zeolite framework type; Right - cage of the gismondine structure showing favored $\mathrm{CO}_{2}$ molecule sites. ${ }^{242}$

As an alternative to considering the full natural tiling units of zeolite frameworks, smaller structural units have also proved useful in characterizing adsorption properties. Martin et al. ${ }^{237}$ carried out a screening of hypothetical zeolite structures in which, from a small set of structures, they first identified sections of the pore volume which contained strong binding sites for $\mathrm{CO}_{2}$, so called "sweet spots", which correlated with high values of the simulated $\mathrm{CO}_{2}$ Henry coefficients and heats of adsorption. From this initial training set, it was detected that the sweet spots corresponded to certain substructural units comprising $8 \mathrm{~T}$-atoms (Fig. 6.1.4), of which two were necessary to define 
a binding site, enclosing each end of the linear molecule. Based on the principle that similar chemical structures have similar chemical properties, screening a much larger database for potentially good $\mathrm{CO}_{2}$ adsorbers thus involves the relatively trivial task of searching for these substructural fragments, greatly reducing the number of materials which need fuller computational characterization.

a)
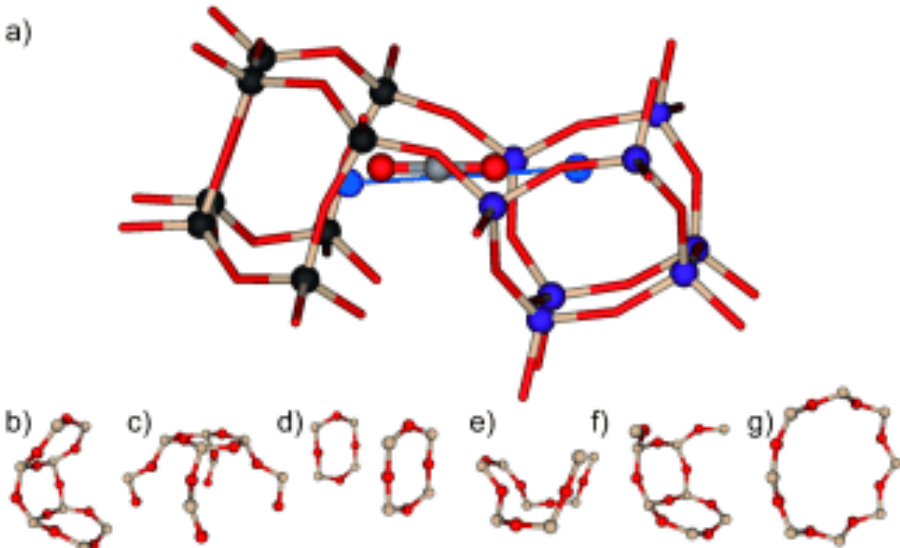

Figure 6.1.4 : (a) Two substructural features (black, purple) detected in a hypothetical zeolite. A vector between features (blue) enables estimation of the $\mathrm{CO} 2$ binding position. The minimumenergy position for $\mathrm{CO}_{2}$ obtained from simulation lies approximately along this vector; $b$ )-g) all six $\mathrm{CO}_{2}$ binding site features identified in a diverse set of hypothetical zeolites. Reprinted with permission from ref. ${ }^{237}$.

Screening of the PZEF database ${ }^{88,90}$ of around one million hypothetical zeolites revealed some 30000 which exhibited the known structural features, a saving well of robbover an order of magnitude in terms of the materials needing to be examined in detail. Recent years have also seen efforts to improve the accuracy of force fields used in the Monte Carlo simulations. Calculating bulk properties such as Henry coefficients, adsorption isotherms and enthalpies is invariably carried out using GCMC in which simple empirical force fields are employed using 2-parameter Lennard-Jones potentials and fixed partial atomic charges on both sorbates and host framework. Inevitably the simplicity of the model is a compromise with the speed and capacity of the modelling technique. Compared to, for instance, hydrocarbons (of which more in a later section), the assumptions of the model are more critical in the case of $\mathrm{CO}_{2}$ adsorption due to the importance of electrostatic effects. $\mathrm{CO}_{2}$ has a significant static quadrupole moment and the $\mathrm{C}-\mathrm{O}$ bonds are strongly polarizable. This suggest that local variations of charge distribution, could have a non-negligible effect on $\mathrm{CO}_{2}$ adsorption properties. The increasing use of periodic DFT as a mainstream technique also means that it is now easily possible to use DFT to derive and calibrate force field models from "first principles". In addition to the force fields already mentioned, Sholl and co-workers recently developed force fields for adsorption in zeolites based on periodic DFT. For $\mathrm{CO}_{2}$ in purely siliceous 
zeolites ${ }^{243}$ a force field based on dispersion-corrected DFT-D2 ${ }^{244}$ calculations gave the best agreement with experiment for a range of siliceous zeolites. When considering sodium-exchanged aluminosilicate zeolites however, it was found that DFT-D2 overestimated the strength of interaction with $\mathrm{CO}_{2} .{ }^{245}$ Instead a force field was derived from calculations using the DFT/CC method ${ }^{246}$ which was able more correctly to describe the interaction with dual-cation sites, and hence was more accurate in reproducing adsorption data in the cationic zeolites. Fischer and Bell ${ }^{242}$ derived a set of charges for framework atoms from DFT calculations using the REPEAT method ${ }^{247}$, together with associated Lennard-Jones parameters. This force field performed effectively in reproducing experimental data and in identifying optimal structure types for $\mathrm{CO}_{2}$ capture as already described. The drawback is that the each individual framework atom can have a different charge, and that the charges thus need to be derived separately for each zeolite to be studied. A set of generic charges was subsequently fitted so as to give the closest possible fit to DFT-D interaction energies in a range of zeolites, but using the same short-range parameters as with the REPEAT charges. ${ }^{248}$ The direct comparison between GCMC simulations using the two sets of charges showed qualitative agreement in terms of comparting the isotherms and related data for different zeolites, but, quantitatively, there were discrepancies of between 10 and $30 \%$ in calculated datasets, with the generic charge model tending to underestimate the strength of $\mathrm{CO} 2$ adsorption. The results thus strongly indicate that, although generic charges are likely to be adequate for initial screening simulations, for more detailed accurate and simulations of $\mathrm{CO}_{2}$ adsorption in zeolites, system-specific charges should be used in future.

We end this case study with the observation that in the past, most studies on CCS have focused on the separation of $\mathrm{CO}_{2}$ and $\mathrm{N}_{2}$, the two main components in post-combustion exhaust gases. Recently, more attention is given to the co-adsorption of other components in the gas stream, such as $\mathrm{H}_{2} \mathrm{O}{ }^{249}, 250, \mathrm{SO}_{x}$ and $\mathrm{NO}_{x}$. ${ }^{251}$ Although the concentration of these molecules is significantly lower than $\mathrm{CO}_{2}$, they can interact strongly with available adsorption sites in zeolites.

Molecular simulations in the cation-exchanged zeolite 13X (FAU topology with Si/AI ratio of 1.24 and $\mathrm{Na}^{+}$countercations) show that this is indeed the case. ${ }^{252}$ In this theoretical study, the Lennard-Jones parameters for $\mathrm{H}_{2} \mathrm{O}$ (3-site SPC/E model ${ }^{253}$ are obtained by scaling the $\mathrm{CO}_{2}$ parameters from the Calero force field. ${ }^{254}$ This simple model is able to reproduce experimental $\mathrm{H}_{2} \mathrm{O}$ isotherms for $13 \mathrm{X} .{ }^{255}$ There is a steep rise in the $\mathrm{H}_{2} \mathrm{O}$ adsorption isotherm, that be ascribed to the strong interaction at low loadings between $\mathrm{H}_{2} \mathrm{O}$ and $\mathrm{Na}^{+}$and the weaker interactions at high loadings, when $\mathrm{H}_{2} \mathrm{O}$ is absorbing in extensive hydrogen bond network. Moreover, the GCMC simulations show that whereas $\mathrm{Na}^{+}$is located close to the $\mathrm{Al}$ centers in the dry material, $\mathrm{H}_{2} \mathrm{O}$ solvates the cations and moves them to the middle of the pore. When adsorbing a $\mathrm{CO}_{2} / \mathrm{H}_{2} \mathrm{O}, \mathrm{H}_{2} \mathrm{O}$ adsorbs close to its pure component isotherm, 
leaving almost no adsorption sites for $\mathrm{CO}_{2}$. Overall, $\mathrm{H}_{2} \mathrm{O}$ reduces the $\mathrm{CO}_{2}$ uptake by an order of magnitude, which was also obtained from an experimental study. The use of this material for industrial post-combustion CCS is therefore seriously hampered and energetically expensive drying operations may be necessary to provide enough performance. An elegant way to reduce this energy penalty is to exploit the temperature-dependent entropy-driven adsorption of $\mathrm{CO}_{2}$ and $\mathrm{H}_{2} \mathrm{O}$ in zeolites. Adsorption of $\mathrm{CO}_{2}$ at high temperature and desorption of $\mathrm{CO}_{2}$ at low temperature could open new perspectives to overcome the competitive adsorption with $\mathrm{H}_{2} \mathrm{O}$ with the power plant's waste heat.

In summary, molecular modelling tools continue to provide a great deal of insight into adsorption processes in zeolites, in particular relating to $\mathrm{CO}_{2}$ capture, as evidenced by many recent advances. They can be used successfully in high-throughput material screening, for instance in determining the minimal parasitic energy for $\mathrm{CCS}$, and in identifying the ideal range for the $\mathrm{CO}_{2}$ Henry Coefficient and investigating the influence of the Si/Al ratio. Secondly, structural features, and particularly the recently-introduced concept of natural tiling, can be employed in a rational search for the optimal sorbent material. Further, recent advances in DFT have enabled a more powerful critique of traditional force field models, and pointed the way to more accurate simulations using systemspecific charges and new parameterizations. Finally, simulations can help to understand the influence of molecules other than $\mathrm{CO}_{2}$ and $\mathrm{N}_{2}$ on the CCS performance.

\subsection{Conversion of hydrocarbons over zeolitic solid acids}

\subsubsection{The nature of intermediates and adsorption complexes within zeolite catalysis}

Any zeolite catalyzed reaction consists of a sequence of steps as schematically depicted in Figure

6.2.1. First the reacting molecules enter the pores of the material where they may diffuse along the zeolite cavities, adsorb at various places and eventually reach the catalytically active site. It must be emphasized that a molecule must be reasonably close to the active site to start the catalytic reaction. This is not always trivial in cases where the molecules have only a weak interaction with the acid site, or where the potential energy surface is relatively flat exhibiting many adsorbed states. After the reaction has taken place and the products are formed, the latter must desorb and can leave the material. Apart from retrieving insight into the adsorption, it is also not evident to obtain insight into the nature of the pre-reactive complex and intermediates solely from experiment. Some intermediates are short-lived and may easily undergo transformations which are hard to follow from an experimental viewpoint. 


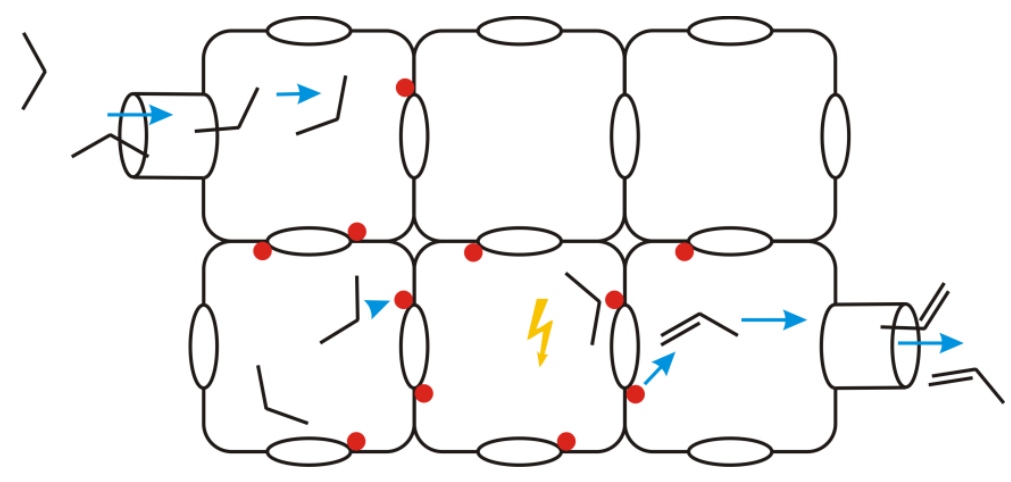

Figure 6.2.1 : Pathway of the molecule in the porous network. After the alkane enters the porous structure, it can diffuse along the cavities, it can reach an active site, where the reaction can take place with formation of the product. The product can desorb and finally leaves the zeolite.

Catalytically active sites are schematically represented by red circles.

In this section we will discuss some aspects related to the accurate description of adsorbed states of molecules within the zeolite and on the existence of intermediates. For this purpose we will concentrate on the adsorption of alkanes and intermediates observed during alkene cracking. This selection is inspired by the fact that an accurate description of the adsorption of alkanes has been shown very challenging from theory as their interaction with the zeolite walls and Brønsted acidic site is governed by long range dispersion interactions which are weak and very difficult to describe theoretically. More in-depth discussion on the process of alkane cracking will be given in section 6.2.2.

On the other hand alkene adsorption is very difficult to track experimentally, as these hydrocarbons are highly reactive even at low temperatures. When an alkene interacts with the Brønsted acidic site, it forms a $\pi$-complex, which may be protonated yielding a chemisorbed species (Figure 6.2.2). The nature of the chemisorbed species is still debated, as it might occur both in a covalently bound alkoxide species or carbenium ion. Kissin reviewed in 2001 possible reaction mechanisms and stated that although the mechanism of catalytic cracking is based on carbenium ions and might at first sight seem relatively simple, many issues and questions remain. ${ }^{256} \mathrm{~A}$ wealth of theoretical data is available on the topic ${ }^{257-261}$, but we focus herein on the adsorption and nature of the intermediates occurring during alkene cracking. ${ }^{262}$ 


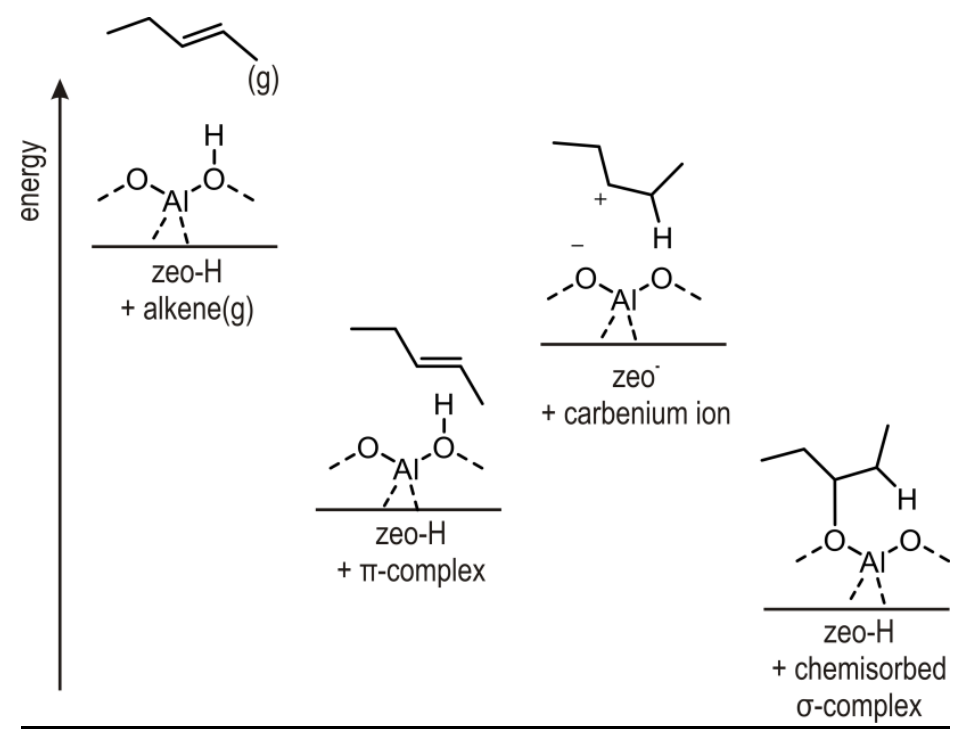

Figure 6.2.2 : Different complexes that can be formed by an alkene in the presence of an acid site. The stability of the complexes is assumed hypothetically.

\section{A. Adsorption of alkanes in zeolites}

The physisorption strength of alkanes is known to increase linearly with the carbon number.

Furthermore, the values of the physisorption energies depend strongly on the size and shape of the zeolite pores. Upon adsorption the entropy of the guest molecule is reduced, the entropy loss has been found to be larger in more confined zeolites. ${ }^{263-265}$

Simulations on the adsorption of alkanes have been performed very extensively in literature using a variety of theoretical models. A first major distinction can be made based on the level of theory used to determine the energy of the system, which can either be based on classical force fields or quantum mechanical methods. Some methodologies use also a hybrid form of these methods, in which part of the system is described using QM methods and the rest of the system at the molecular mechanics (MM) or classical force field based level. An excellent review was written by Smit and Maesen on the usage of force fields for the molecular simulation of zeolites and their applications. 266 The advantage of a force field description relates to its computational efficiency, which enables the description of larger systems within a reasonable computational time. As these methods are however based on parameters which have been determined on basis of equilibrium configurations or based on experimental data fitting, these methods are not suited to describe chemical reactions in which bonds are broken or formed. Recently some interesting developments occurred in the field of reactive force fields which are able to describe reactive processes also. The methods have not been used widespread within zeolite catalysis and the accuracy depends on the parameters inserted 
in the force fields. A detailed description is beyond the scope of this review but we refer to some works on the application of these methods in the field of zeolite catalysis. ${ }^{267,268}$

The usage of quantum mechanical (QM) based simulations has also been widely adopted using a variety of models ranging from layered methods, to cluster methods and periodic models. Quantum mechanical based methods are far more expensive than classical methods but enable a consistent description of the adsorption and reactive steps. Especially for the description of adsorption care needs to be taken in treating the long-range dispersion interactions appropriately. [vide infra] When using QM methods a further major distinction can be made in the methods adopted to describe the zeolite environment. Either a cluster based model may be used in which part of the zeolite is cut out from the periodic structure or a periodic model in which the material is simulated using periodic boundary conditions. ${ }^{269,270}$ Earlier theoretical papers often used very small clusters in which the topology of the material was not properly taken into account for reasons of computational expense. ${ }^{271,272}$ The periodic model is in principle the most natural way to simulate the molecular environment but also comes at a serious computational cost. During the last decades many successful simulations have also been performed using an extended cluster model in which a large portion of the zeolite is taken into account. ${ }^{273-278}$ In most cases, not all atoms are described at the same level of theory and a layered approach is used in which part of the system is described very accurately and the rest is described at a lower level of theory. These methods are often referred to as $\mathrm{QM} / \mathrm{QM}, \mathrm{QM} / \mathrm{MM}, \ldots$ schemes. Some visual representations of the various possible molecular models are shown in Figure 6.2.3. It is not our intention to give a more detailed review on these simulation methods here, but we refer to a recent review paper by some of the present authors on the subject. ${ }^{279}$
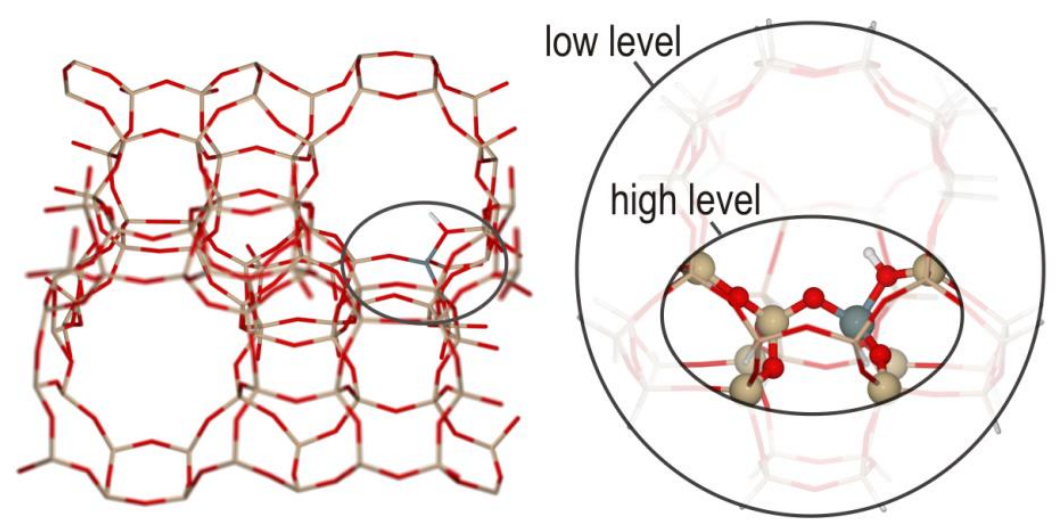

Figure 6.2.3 : Illustration of various models to account for the zeolite topology : (a) a periodic model for the H-ZSM-5 structure (b) a 46 T cluster for H-ZSM-5 with indication of various regions which may be simulated at different levels of theory according to appropriate hybrid schemes. 
Long-range dispersion interactions The accurate description of adsorption by quantum mechanical methods has for a long time been hampered by the difficulty in describing long range electron correlation effects, which are very difficult to describe accurately by theoretical methods. It is certainly not possible to give a complete compilation of all literature on the topic, but we will highlight some articles to show the strengths and weaknesses of various methods which are currently available.

Within the framework of periodic DFT based methods, early pragmatic solutions were proposed to account for the van der Waals (vdW) interactions by Hafner et al. ${ }^{280}$. They proposed to correct the periodic DFT energies for van der Waals interactions using an add-on Lennard-Jones potential between the hydrocarbon atoms and the framework oxygen, silicon and aluminum atoms. Indeed DFT methods are very popular for the treatment of large systems but local density functionals are not able to account for long-range electron-electron correlation effects. The DFT-D methodology proposed by Grimme has become very popular in which a damped dispersion term is added to standard functionals such as PBE, B3LYP,... 244, 281-283 The correction terms contain parameters which have been fitted for various different functionals. More advanced methods have been developed for the estimation of the inter-atomic coefficients which are parameter-free, such as the methods proposed by Tkatchenko and Scheffler. ${ }^{284}$

Very recently a very detailed study was performed by Goltl et al. on the influence of van der Waals interactions between hydrocarbon molecules and zeolites. ${ }^{285}$

A compilation of their adsorption energies for methane, ethane and propane are given in Table 6.2.1. By substitution of a Si atom by an Al atom, a formally negative charge is created which is compensated by a BAS to one of the four oxygen atoms next to the Al atom. Four possibilities for the position of the BAS are possible as schematically shown in Figure 6.2.4. Only very small energy differences were obtained between the various acid sites, although the exact position of the acid site has an influence on the adsorption energy as shown in Table 6.2.1. This is partly related to the strength of the acid site but also to the possible conformations the adsorbate can adopt with respect to the various acid sites. In some cases the long-range interactions will contribute larger as the molecule can adopt a conformation which optimizes the strength of the vdW interactions. 


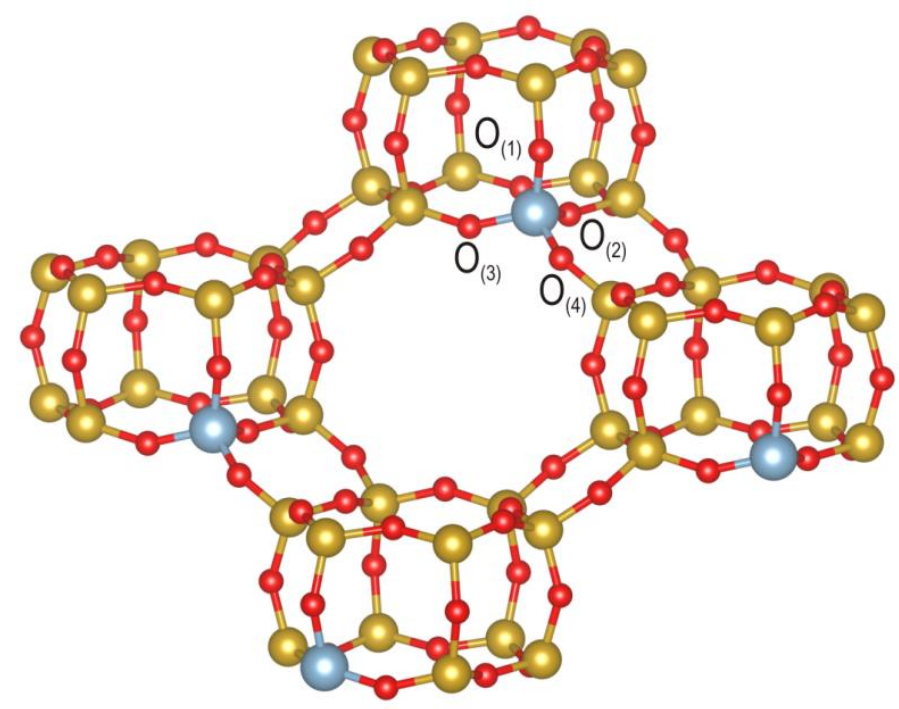

Figure 6.2.4 : Four unit cells of chabazite. All T-sites are geometrically equivalent and surrounding by four $\mathrm{O}$ atoms labelled $\mathrm{O}(1)$ to $\mathrm{O}(4)$. A Brønsted acid site is created by attaching a hydrogen atom to an oxygen site bound to $\mathrm{Al}$. Si atoms are visualized in orange, $\mathrm{Al}$ atoms in dark-grey and $\mathrm{O}$ atoms in red. Adapted from ref. ${ }^{285}$.

Table 6.2.1 shows some general trends: The periodic DFT calculations using the PBE functional but no additional correction terms for the dispersion interactions are not suited to describe adsorption processes. Given their computational efficiency the PBE-D methods give reasonable estimates of the adsorption energies with average errors of about $5 \mathrm{~kJ} \mathrm{~mol}^{-1}$ compared to the experimental values. This statement is true provided that the finite temperature effects are accounted for. [vide infra] This is at least very important for host-guest interactions which are controlled by the dispersion interactions.

Apart from the addition of these damped dispersion terms, there is a continuous effort undertaken by various groups to develop more accurate functionals. For adsorption of alkanes in zeolites, the work of Brogaard et al. is interesting as they developed a functional called BEEF-vdW, that gave an accurate description of physisorption of n-alkanes in ZSM-22. ${ }^{286}$ This functional was developed to describe vdW interactions and chemical bond formation correctly and was implemented for DFT calculations with periodic boundary conditions. Various groups have developed advanced functionals to describe weak interactions playing a prominent role in many host-guest systems. The most well-known example is M06-2X developed by the Truhlar group, which has been used by diverse authors to study adsorption of various compounds in zeolites. ${ }^{287-292}$ For more detailed assessments of various exchange correlation functions and their performance to describe the adsorption of alkenes we refer to the work of Gomes and co-workers, who performed a benchmark 
study on the adsorption enthalpies of various light hydrocarbon molecules in MFI-based zeolites using various exchange correlation functionals and adopting a $\mathrm{QM} / \mathrm{MM}$ approach on clusters of varying size. ${ }^{293}$

Apart from DFT based methods, a range of computationally very expensive methods are available such as Møller Plesset perturbation theory (MP2) or methods where the exact exchange is combined with correlations treated in the random phase approximation (RPA). ${ }^{294-296}$ For a more detailed description of these methods we refer to the original article of Goltl and co-workers. ${ }^{285}$ For the time being, these computationally very expensive methods such as RPA methods are only applicable within zeolitic systems whose geometries have been optimized at a lower level of theory. For the dataset tested by Goltl et al. ${ }^{285}$ (Table 6.2.1) the RPA method underestimates the adsorption energy by about $8 \mathrm{~kJ} \mathrm{~mol}^{-1}$ whereas the MP2 post-Hartre- Fock method gives an overestimation of about $4 \mathrm{~kJ}$ $\mathrm{mol}^{-1}$ (averaged over methane, ethane and propane).

Table 6.2.1 : Adsorption energies of methane, ethane, and propane in acid chabazite [in kJ mol${ }^{-1}$ ], obtained from fully selfconsistent calculations using DFT methods, and from single-point calculations for the PBE-D geometry using the RPA, RPA-HF, and MP2 methods. The calculations have been performed for acid protons attached to the $\mathrm{O}(1)$ to $\mathrm{O}(4)$ oxygen atoms. Ensemble averages over MD trajectories at $300 \mathrm{~K}$ are also taken up at the PBE-D level. Results are taken from ref. ${ }^{285}$

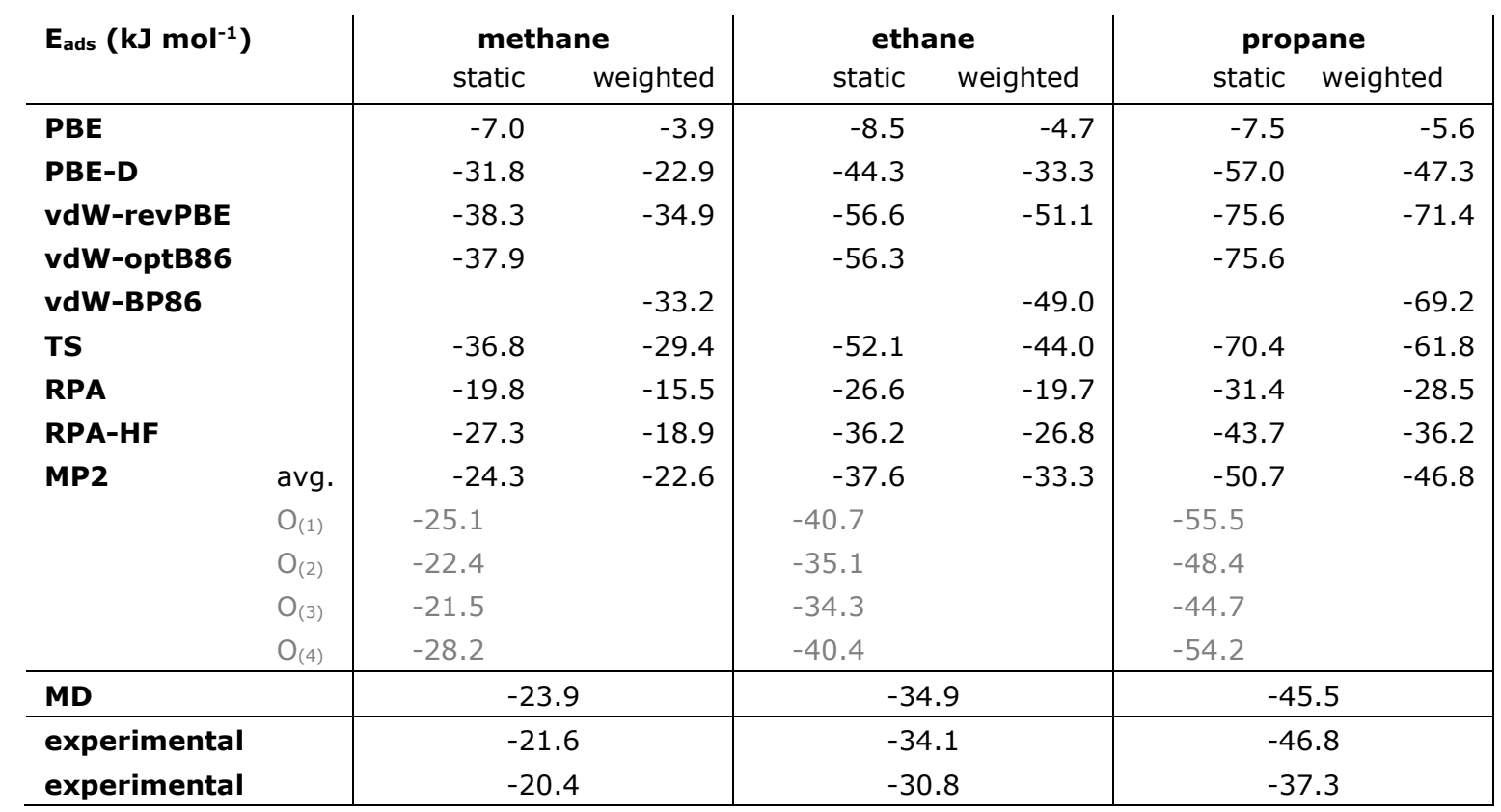

Seminal work has also been performed by Sauer and co-workers on the development of accurate models to describe the adsorption and reaction enthalpies. Tuma and Sauer introduced a multistep MP2:DFT approach, which combines MP2 calculations with Gaussian basis sets for the reaction site 
and plane-wave DFT for the full system under periodic boundary conditions. ${ }^{297}$ The hybrid scheme produces an approximate MP2 energy estimate of the system with periodic boundary conditions and aims to take into account the long-range crystal potential as well as steric effects caused by confinement within the pores of zeolitic materials. ${ }^{297,298}$ The method produces very accurate energetic results but is computationally very demanding and can certainly not be regarded as the method of choice for daily applications. ${ }^{299}$

Another method developed by Sauer and co-workers which is much more attractive to study the adsorption is the QM-Pot methodology. ${ }^{300}$ The method relies on a quantum mechanics interatomic potential functions approach and the periodic molecular mechanics calculations are performed using a DFT-parametrized core-shell model for the zeolite. ${ }^{301}$ The method has been successfully applied to study the adsorption of hydrocarbons in a variety of industrially relevant zeolites. ${ }^{302-305}$ De Moor et al. extended the DFT parametrized force field used in the QM-Pot program with carbon-carbon, carbon-hydrogen and alkoxy bond describing parameters, to describe accurately interactions of hydrocarbons with zeolite frameworks. Their results for the physisorption energies of $\mathrm{C} 4$ to $\mathrm{C} 10$ species in H-FAU using the QM-Pot(MP2//B3LYP) methodology are shown in Figure 6.2.5 in terms of the carbon number.

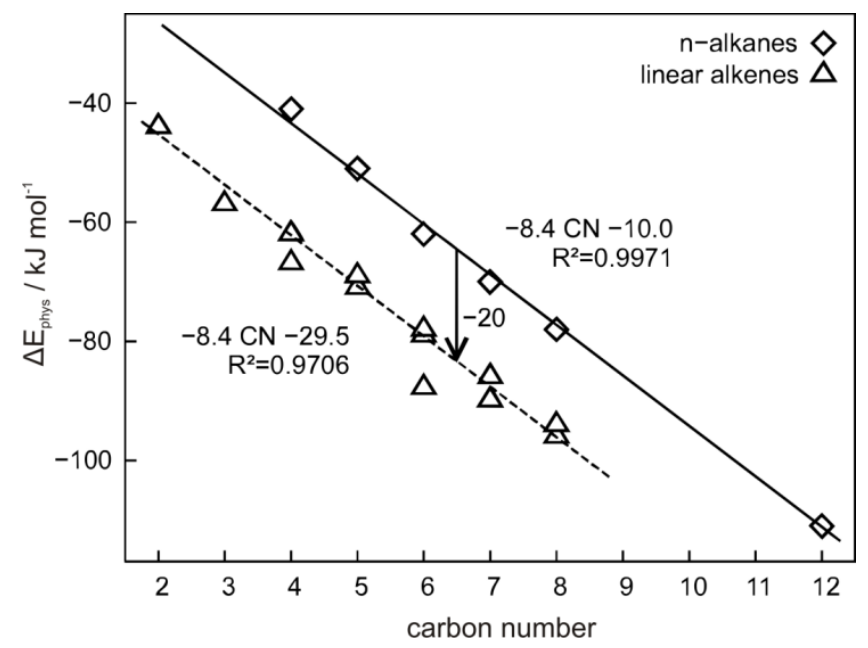

Figure 6.2.5 : Physisorption energies of $n$-alkanes and linear alkenes in H-FAU as a function of the carbon number. The $\pi$-complex generates a contribution of $-20 \mathrm{~kJ} \mathrm{~mol}^{-1}$ to the alkene physisorption energies. Reprinted with permission of ref. ${ }^{303}$.

The physisorption energy varies from $-41 \mathrm{~kJ} \mathrm{~mol}^{-1}$ for $\mathrm{n}$-butane to $-111 \mathrm{~kJ} \mathrm{~mol}^{-1}$ for dodecane in $\mathrm{H}$ FAU. The physisorption energy indeed increased linearly with the alkane chain length, with an increased physisorption energy of about $8.4 \mathrm{~kJ} \mathrm{~mol}^{-1}$ per carbon atom. A weak electrostatic interaction is present between the acid proton and the polarized $\mathrm{C}-\mathrm{H}$ bond of the alkane of the order of $5 \mathrm{~kJ} \mathrm{~mol}^{-1}$. Eder and Lercher reported from their experimental studies a contribution of $6 \mathrm{~kJ} \mathrm{~mol}^{-1}$ in FAU and $10 \mathrm{~kJ} \mathrm{~mol}^{-1}$ in MFI arising from the acid sites to the sorption enthalpy of alkanes. ${ }^{306}$ The 
values obtained with the QM-Pot methodology are in very good agreement with these experimental values. Pantu et al. found an extra stabilization of $6.2 \mathrm{~kJ} \mathrm{~mol}^{-1}$ per carbon atom using an ONIOM(MP2//B3LYP:UFF) model, which is also in reasonable agreement with the QM-Pot values. ${ }^{274}$ On a side note it may be remarked that physisorption energies of alkenes are typically $20 \mathrm{~kJ} \mathrm{~mol}^{-1}$ larger than those of alkanes of similar chain length (see Figure 6.2.5), due to the formation of a stable $\pi$-complex with the BAS and the alkene double bond for alkenes. More information on alkene sorption will be given in the next section.

This short summary of methods shows that there is a range of computational methods available which indeed succeed in describing the adsorption of alkanes reasonably well, provided that dispersion effects and the zeolite topology is properly taken into account.

\section{Temperature effects}

Alkanes show a very weak interaction with the BAS and can adopt many orientations and conformations in the pores of the zeolite material. The limitation of estimating adsorption enthalpies based on a single configuration was become clear from the values reported by Rosch et al. ${ }^{307}$. They calculated the adsorption enthalpies of a series of C3-C6 alkanes in silicalite and H-ZSM5 using periodic PBE-D and a nonlocal correlation functional vdW-DF2 ${ }^{308}$. The results are shown in Table 6.2.2 together with the experimental data and data obtained from other theoretical methods. The authors conclude that the adsorption energies are seriously overestimated (ranging from 21-46 $\mathrm{kJ} \mathrm{mol}^{-1}$ ). The reported values however show that the adsorption energy may be about $11-20 \mathrm{~kJ} \mathrm{~mol}^{-}$

${ }^{1}$ less exothermic, if another stable minimum on the potential energy surface is taken. To enable a reasonable comparison with experimental data for molecules that exhibit only a very weak adsorption mode, the conformation space of the zeolite needs to be sampled sufficiently.

Table 6.2.2: Comparison of experimental adsorption enthalpies $\mathrm{H}_{\text {ads }}$ and calculated adsorption energies $E_{\text {ads }}$ of alkanes on $\mathrm{HZSM}-5\left(\mathrm{~kJ} \mathrm{~mol}^{-1}\right)$ from various computational approaches. Adsorption in straight (S) and sinusoidal (Z) channels. Experimental values from a) ref. ${ }^{309}$ and b) ${ }^{306}$. Theoretical results taken from ref. ${ }^{307}$ except c) ref. ${ }^{302}$ and d) ref. ${ }^{310}$

\begin{tabular}{|c|c|c|c|c|c|c|c|c|c|}
\hline \multicolumn{2}{|c|}{$E_{\text {ads }}\left(\mathrm{kJ} \mathrm{mol}^{-1}\right)$} & \multicolumn{2}{|c|}{ Hads } & \multicolumn{2}{|c|}{$E_{\text {ads }}$} & \multicolumn{4}{|c|}{ Eads } \\
\hline & mode & $\underset{\text { [a] }}{\text { Exp. }}$ & $\begin{array}{c}\text { Exp. } \\
\text { [b] }\end{array}$ & $\begin{array}{c}\text { QM-Pot } \\
{[c]}\end{array}$ & PBE-D & $\begin{array}{l}\text { vdW- } \\
\text { DF2 }\end{array}$ & PBE-D & PBE & \\
\hline \multirow[t]{2}{*}{ propane } & $\mathrm{S}$ & -41 & $-46 \pm 1$ & -52 & & -55 & -46 & -3 & -43 \\
\hline & Z & & & -63 & -48 & -67 & -68 & -8 & -61 \\
\hline \multirow[t]{2}{*}{ butane } & $S$ & -52 & $-58 \pm 1$ & -65 & & -69 & -58 & -2 & -56 \\
\hline & Z & & & -84 & -63 & -87 & -83 & -4 & -79 \\
\hline \multirow[t]{2}{*}{ pentane } & S & -63 & $-70 \pm 1$ & -85 & & -80 & -69 & 4 & -74 \\
\hline & Z & & & -96 & -80 & -97 & -91 & 10 & -101 \\
\hline \multirow[t]{2}{*}{ hexane } & S & -72 & $-82 \pm 1$ & -101 & & -92 & -75 & 17 & -93 \\
\hline & Z & & & -106 & -92 & -118 & -104 & 22 & -126 \\
\hline
\end{tabular}


The measured values are a result of an average of all structures visited at the temperatures of the experiment. De Moor et al. studied the influence of temperature on the adsorption entropies beyond the harmonic oscillator approach, however still relying on one configuration on the potential energy surface. ${ }^{133} 311$ They showed that it was important to replace the frequency contributions originating from translational or rotational motions of the adsorbate in the partition function by free translational and rotational motions. Their approach was based on the Mobile Block Hessian (MBH) approach developed by Ghysels et al. 134,311,312. The method is valuable as it shows the importance of soft vibrational modes of the adsorbate relative to the zeolite, but still ignores the possibility that the interaction between the hydrocarbon and acid site may be broken at higher temperatures. The temperature-dependence of the adsorption energy was recently studied by Goltl and coworkers using MD simulations. ${ }^{285}$ Periodic boundary conditions were applied using the PBE-D functional to study the methane, ethane and propane in protonated chabazite at $300 \mathrm{~K}$. At room temperature there is a non-negligible probability that the alkane desorbs from the acid site and moves freely through the pores of the zeolite. A good agreement was obtained with experimental values, using an approach where the adsorption energies obtained for complexes close to the acid site and complexes in the purely siliceous version of the material were weighted according to their probabilities of being close to the acid site. The resulting values are shown in Table 6.2.1. It is very interesting to note that the dynamically averaged values are systematically smaller compared to the OK values. As most processes such as cracking occur at high temperatures, it is crucial to account for such effects. The general drawback of these methods however is the excessively long simulation times needed to obtain converged values for the adsorption enthalpies directly from MD simulations. An alternative is the approach followed by Goltl et al. and to work with weighting factors which may be obtained from shorter simulations of the order of 20 ps.

For molecules for which a more specific interaction exists with the acid site such as water, alcohols, nitriles, the effects may be less pronounced. In that case the interaction is much stronger, preventing the molecule from a free walk in the pores of the zeolite. Indeed the comparison between experiment and methods relying on only one minimum is much better in those cases. ${ }^{288,}$ 299, 307 More extensive studies are necessary in the future to assess the influence of temperature on the overall adsorption behavior of molecules in zeolites. An effect that may also occur is the formation of complexes of various guest molecules. More information on this topic can be found in refs. ${ }^{279,313}$. 


\section{B. Alkene adsorption : Physisorption versus chemisorption and persistent carbenium ions}

The study of alkene adsorption is very challenging both from theoretical and experimental side due to their high reactivity. As introduced in the onset of section 6.2 various adsorption complexes may occur upon interaction with the BAS: a $\pi$-complex, a chemisorbed species for which the nature may be an alkoxide or carbenium ion. Some landmark papers are briefly reviewed hereafter.

The adsorption of $\mathrm{C} 2-\mathrm{C} 5$ species in H-FER has received considerable attention. H-FER is an efficient catalyst for the skeletal isomerization of linear butenes and has been used commercially. ${ }^{26,27,314}$ The stability of various C4 species and especially the relative stabilities of the physisorbed, carbenium ions and various alkoxides in H-FER received considerable attention. Table 6.2.3 shows a summarizing table for the energies of the species shown in figure 6.2.6 (physisorbed isobutene, tertbutoxide, the tert-butyl carbenium ion and the isobutoxide) using various methodologies.
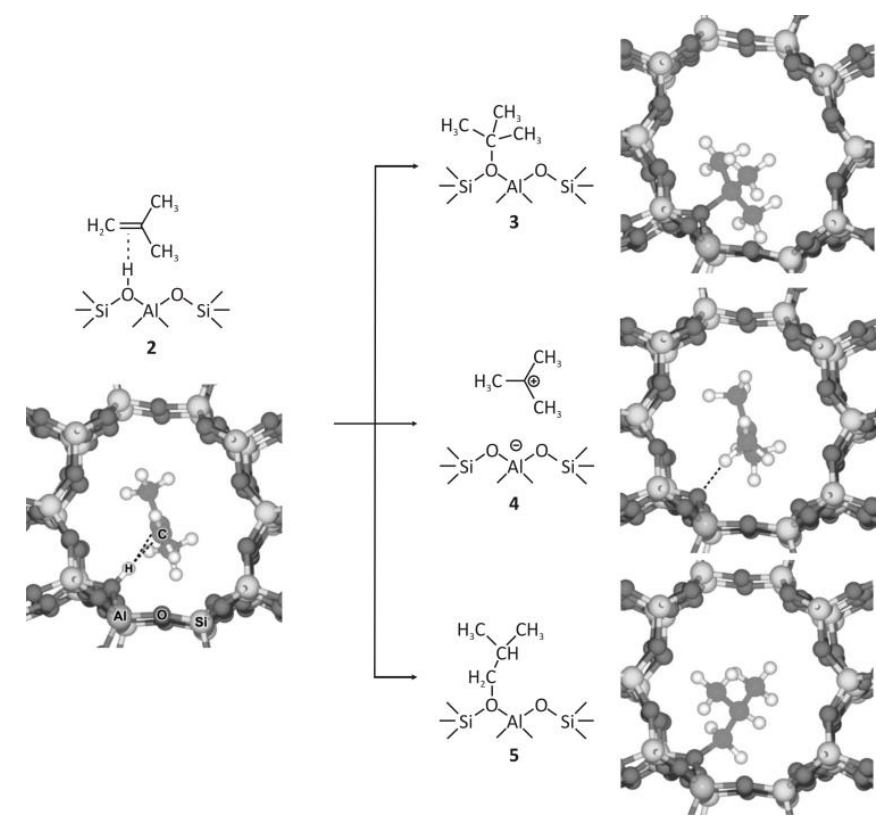

Figure 6.2.6 : Protonation of isobutene in H-FER. Structure cut-outs of the $\pi$-complex of isobutene in ferrierite $\mathbf{2}$, the tert-butoxide $\mathbf{3}$, the tert-butyl carbenium ion $\mathbf{4}$, and the isobutoxide $\mathbf{5}$. Hydrogen bonds are shown by dashed lines. Adapted from ref. ${ }^{298}$.

Table 6.2.3 : Physisorption and chemisorption energies of isobutene in H-FER. (All energies are reported in $\mathrm{kJ} \mathrm{mol}^{-1}$ )

\begin{tabular}{|l|l|l|l|l|}
\hline & $\begin{array}{l}\text { QM-Pot } \\
\text { (MP2//B3LYP)[a] }\end{array}$ & $\begin{array}{l}\text { QM-Pot(B3LYP) } \\
{[\mathrm{b}]}\end{array}$ & $\begin{array}{l}\text { Final MP2 } \\
\text { estimate [c] }\end{array}$ & $\begin{array}{l}\text { PBE } \\
{[\mathrm{c}]}\end{array}$ \\
\hline $\begin{array}{l}\text { Physisorbed } \\
\text { isobutene }\end{array}$ & -79 & -49 & -78 & -62 \\
\hline Tert-butoxide & -67 & -62 & -48 & -67 \\
\hline Iso-butoxide & -94 & -145 & -73 & -70 \\
\hline $\begin{array}{l}\text { Tert-butyl } \\
\text { carbenium ion }\end{array}$ & na & na & -21 & -29 \\
\hline
\end{tabular}

[a]Taken from ref. ${ }^{304}$ 
[b]Taken from ref. ${ }^{315}$

[c] Taken from ref. ${ }^{298}$ Final MP2 estimate corresponds to the energy for the full periodic system at the complete basis set level and obtained using the multistep MP2:DFT procedure introduced by Tuma and Sauer.

The values referred to as QM-Pot(B3LYP) used DFT based methods for the QM part in the hybrid DFT/force-field approach. ${ }^{315}$ In the original publication of Nieminen et al., adopting this approach, interesting insights were obtained on the stability of various adsorbed alkene like species. They found that primary and secondary alkoxide species are significantly more stable than adsorbed alkenes and that the chemisorption energy increases with increasing carbon number. It is found that tertiary alkoxides are much less stable than primary and secondary ones and may even be nonexistent depending on the degree of steric hindrance induced by the zeolitic local environment. In the timeframe of publication the results were very important, but later the relative energies of the C4 species were reevaluated by Tuma and Sauer using the MP2:DFT method, which allows to give a MP2 extrapolated value for periodic systems at the complete basis set level. ${ }^{298}$ For the adsorption of $\mathrm{C} 4$ species in H-FER, following energetic values are found for the $\pi$-complex, the isobutoxide, the tert-butoxide and the tert-butyl carbenium ion : $-78,-73,-48$ and $-21 \mathrm{~kJ} \mathrm{~mol}^{-1}$, which must be compared with the pure DFT values using the PBE functional of $-62,-70,-67$ and $-29 \mathrm{~kJ} \mathrm{~mol}^{-1}$. It is clear that inclusion of dispersion effects is very important and that the correction is not uniform for the different hydrocarbon species. The values given in Table 6.2 .3 show that the stability of carbenium ions versus alkoxide in the particular case for tert-butyl species in H-FER concerns a very subtle interplay of factors. In 2008, De Moor et al. used the QM-Pot(MP2//B3LYP) methodology, in which the DFT parametrized force field in QM-Pot was extended with parameters to describe more accurately the physisorbed and chemisorbed species within zeolites. ${ }^{304}$ It is clear that the extended version of the QM-Pot method with the new version of the force field and new point charge model give adsorption energies closer to the best estimates of Sauer et al.

Later Tuma and Sauer extended their study on the stability of the tert-butyl cation and alkoxide species in H-FER and included for the first time also entropy effects to assess the overall stability of the various intermediates. ${ }^{316}$ The calculations were performed using a periodic model using the PBE functional which did not account properly for the dispersion interactions at that time. Irrespective hereof, important temperature effects were found on the stability of the alkoxide versus the carbenium ion. From the viewpoint of electronic energies, the tert-butoxide was more stable than the tert-butyl cation, but for temperatures higher than $120 \mathrm{~K}$ the carbenium ion becomes more 
stable. The results from this study show that the persistence of intermediates within zeolites depends largely on the conditions and can't be evaluated solely on basis of electronic energies. All static approaches discussed so far, are inherently limited to describe finite temperature effects. A methodology to circumvent the shortcomings of static approaches which use information of a limited number of points on the free energy surface, is the usage of MD based methods. If performed under the right conditions, all minima contributing to the observed adsorption are accounted for. For theoretical description of cracking processes which occur at elevated temperatures, one must realize that observed minima and intermediates on the free energy surface are also dependent on the operating temperature.

In a very recent contribution by some of the presenting authors physisorption of linear pentenes was studied in $\mathrm{H}-\mathrm{ZSM}-5$ using both static cluster and static periodic methods but also using ab initio MD methods. ${ }^{317}$ All calculations in that study were performed using the PBE-D level of theory. A detailed assessment of the conformations of pentene in the gas phase and adsorbed pentene in the zeolite using MD based methods indeed shows that many more geometries are possible in the pores of the zeolite. The histograms of the dihedral angle $\mathrm{C2}-\mathrm{C} 3-\mathrm{C} 4-\mathrm{C} 5$ of 2-pentene in the gas phase and in the zeolite is shown in Figure 6.2.7.

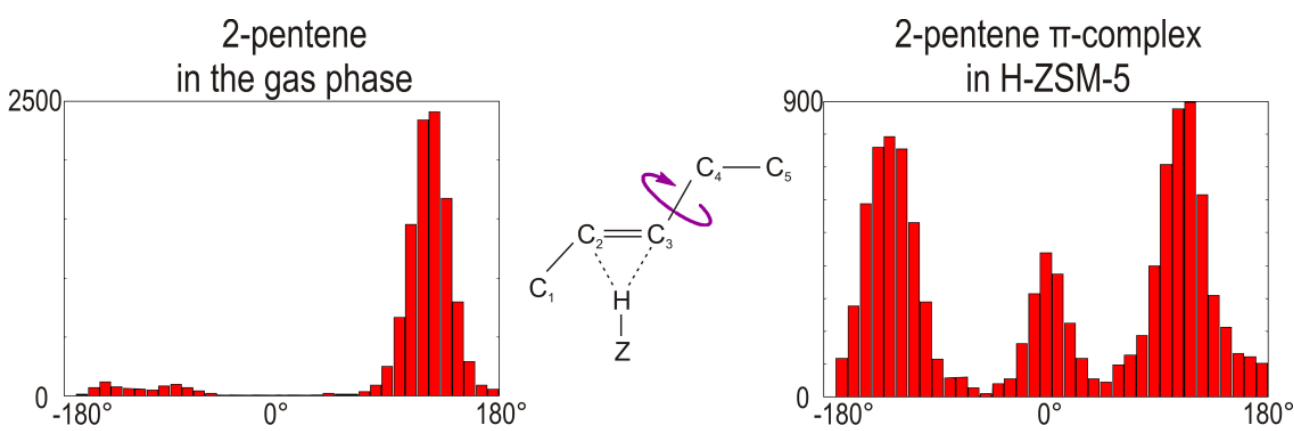

Figure 6.2.7: MD simulation of 2-pentene in the gas phase and in the pores of $\mathrm{H}-\mathrm{ZSM}-5$ forming a $\pi$ complex. Adapted with permission from ref. ${ }^{317}$.

In the same study we also calculated physisorption energies of pentene on basis of the MD calculations, which were in very good agreement with experiment. A downside of the approach is the extensive sampling time necessary to obtain reliable values for the heats of adsorption of hydrocarbons on basis of molecular dynamics based methods. For the case of pentene simulation times of more than 100 ps were necessary using ab initio MD based methods, which is not a routinely applicable method for the calculation of adsorption energies. 


\section{Persistent Carbenium ions}

Experimental evidence for persistent carbenium ions has only been found for cyclic alkenyl or aromatic carbenium ions by NMR, UV/Vis and IR spectroscopy. ${ }^{318-321}$ Computational approaches also contributed to the rationalization of persistent carbenium ions ${ }^{322-324}$ and the transformation of various intermediates into each other. ${ }^{325,326}$

Nicholas and Haw concluded that stable carbenium ions could be observed by NMR provided that the neutral compound from which it originates has a proton affinity (PA) of $875 \mathrm{~kJ} \mathrm{~mol}^{-1}$ or larger. ${ }^{327}$ The seminal statement made by Haw was theoretically founded by quantum mechanical calculations, showing that proton transfer from a H-zeolite to a molecule or a molecular cluster occurs provided that the PA of the molecule is about $854 \mathrm{~kJ} \mathrm{~mol}^{-1}$ or larger, which corresponds to the PA of ammonia. ${ }^{323,328}$

In 2011 Fang et al. performed an extensive study on the effects of zeolite framework on the stability of carbenium ions in zeolites, using an ONIOM based model for the zeolites $\mathrm{H}-\mathrm{ZSM}-5, \mathrm{H}-\mathrm{Y}$ and $\mathrm{H}$ beta. ${ }^{329}$ They indeed showed that the energy difference between the carbenium ion and the $\pi$ complex correlates well with the PA of the neutral hydrocarbons. The correlation plot is shown in Figure 6.2.8 for all carbenium ions generated from the neutral hydrocarbons displayed in Figure 6.2.9. The larger the PA value the more negative the difference between the ion pair (carbenium ion) and $\pi$-complex and the higher the expected stability of the carbenium ion. The carbenium ions $7 c, 8 c, 11 c, 19 c$ and $20 c$ have negative values for the difference in energy between the carbenium ion and the $\pi$-complex and have a PA which is higher than $210 \mathrm{kcal} / \mathrm{mol}$. Indeed these carbenium ions were found experimentally to be persistent in various zeolites, which was proven by solid-state NMR. ${ }^{330-335}$ Experimentally Bordiga and co-workers performed seminal work and showed that the hexamethylbenzenium ion is stable in the zeolite cavities of the beta zeolite at least up to $573 \mathrm{~K}$. The remarkable persistence of this carbenium ion could be attributed to spatial constraints imposed by the tight fit of the cation inside the zeolite channels. Their work was based on a detailed study of the vibrational and electronic transitions. ${ }^{321}$ 


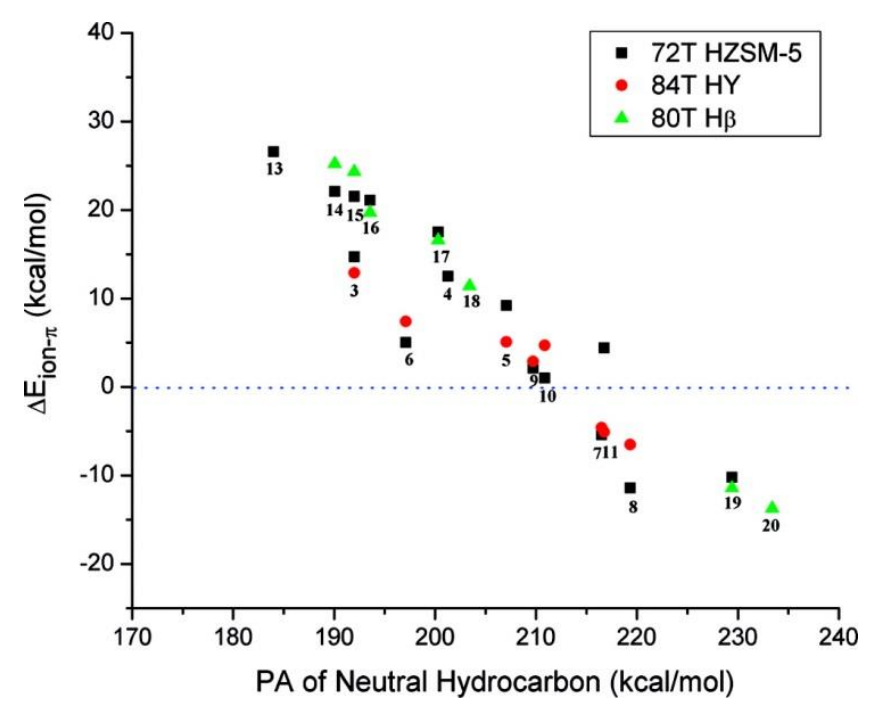

Figure 6.2.8 : Correlation plot of the energy difference between the carbenium ion and the $\pi$ complex with the proton affinity of the neutral hydrocarbons. Reprinted with permission from Fang et al. ${ }^{329}$.

$$
\mathrm{H}_{2} \mathrm{C}=\mathrm{CH}_{2}
$$

$1 \mathrm{~h}$<smiles>C1=CCC=C1</smiles>

$6 h$<smiles>CC1C=C(c2ccccc2)c2ccccc21</smiles>

$11 \mathrm{~h}$<smiles>Cc1cc(C)c(C)cc1C</smiles>

$16 \mathrm{~h}$

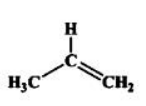

2 h<smiles>CC1=CCC(C)=C1</smiles>

7 h<smiles>c1ccccc1</smiles>

$12 \mathrm{~h}$<smiles>Cc1cc(C)c(C)c(C)c1C</smiles>

17h<smiles>C=C(C)C</smiles>

3h<smiles>CC1=CCC(C)=C1C</smiles>

8 h<smiles>Cc1ccccc1</smiles>

13h<smiles>Cc1c(C)c(C)c(C)c(C)c1C</smiles>

$18 \mathrm{~h}$<smiles>C=Cc1ccccc1</smiles>

$4 h$<smiles>CC1=CCc2ccccc21</smiles>

9h<smiles>Cc1cccc(C)c1</smiles>

14h<smiles>C=C1C=C(C)C(C)(C)C(C)=C1</smiles>

19h<smiles>C=C(C)c1ccccc1</smiles>

5 h<smiles>CC1=CC(C)(C)c2ccccc21</smiles>

$10 \mathrm{~h}$<smiles>Cc1cccc(C)c1C</smiles>

15h

$20 h$

Figure 6.2.9 : Corresponding neutral hydrocarbons of the carbenium ions used in the correlation plot of Figure 6.2.8. Reprinted with permission of ref. ${ }^{329}$. 
Indeed also in the work of Fang and co-workers, some stabilizing effects were found depending on the zeolite framework to facilitate the formation of certain carbenium ions. Furthermore in some cases alkoxy species may be formed provided that the steric constraints around the C-O bond are not too pronounced. Of course one must realize that these observations are made on basis of electronic energies and thus the effect of temperature is not yet accounted for. Furthermore the lifetime of carbenium ions will also depend on the barriers separating the carbenium ions from the alkoxides and the physisorbed species. In view of this, various studies also appeared on the mechanism of skeletal isomerization of butene like species in zeolites. 324, 325, 336-339 Specifically for the tert-butyl carbenium ion, Tuma and Sauer ${ }^{316}$ estimated half life times of several $\mu \mathrm{s}$. An important side note is that these barriers are relatively sensitive to the level of theory used for the calculation of the barriers. Yet irrespective hereof, these lifetimes are probably not long enough for the detection by NMR spectroscopy ${ }^{340}$ but the timescale of UV/Vis spectroscopy might give an alternative for detection of these transient species. ${ }^{341}$

A comprehensive review on NMR spectroscopic studies of hydrocarbon conversions on solid acid catalysts was given by Stepanov A.G. In this review, the possibility to detect various intermediates by NMR spectroscopy is discussed. ${ }^{342}$

An interesting new approach to study transient intermediates in zeolites, which are separated by non-negligible barriers is the usage of enhanced sampling MD based methods which also allow to study transitions between various minima on the free energy surface. Indeed transformations between various intermediates on the free energy surface are mostly activated and thus the probability of sampling such event in a regular MD run of a few ps is very low. To enhance sampling of interesting regions of the free energy surface a multitude of methods has been developed. ${ }^{54}$ It is not our intention to give a detailed discussion on the various advanced MD based methods here. We only give an example of a very recent study performed on the transformation of physisorbed pentene to the alkoxide at $323 \mathrm{~K}$ in H-ZSM-5. We adopted the metadynamics based method and could follow the transformation between all species in such run. The resulting 1D free energy profiles along the lowest free energy paths in Figure 6.2.10.

For more technical details about the simulation we refer to the original article . ${ }^{317}$ There clearly exists a metastable state between the 1-pentene $\pi$-complex and the 2-pentoxide, which was characterized as a carbenium ion. The results obtained so far are capable of giving free energy barriers no transition rates, as the regular dynamics is disturbed by adding Gaussian hills to enhance the occurrence of an active process. Interesting methods have been proposed by theoretical groups to deduce also from these simulations the transition rates but they have not yet been used in zeolite applications. ${ }^{343}$ 


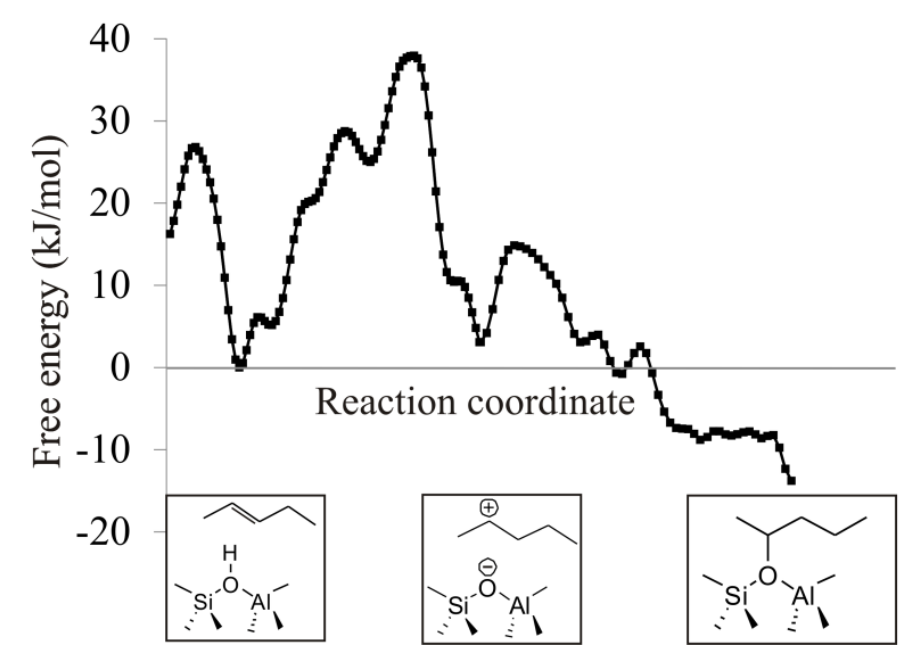

Figure 6.2.10 : 1D free energy profile along the lowest free energy path for the formation of 2pentoxide from a 1-pentene $\pi$-complex resulting from metadynamics simulations at $323 \mathrm{~K}$. Adapted with permission from ref. ${ }^{317}$.

\subsubsection{Alkane cracking : Towards predicting product selectivities and structure activity relationships.}

Brønsted acid catalyzed cracking of alkanes has received broad attention due to its central importance for the production of fuels from crude oil. Even after more than 60 years of intensive research on the topic and given the economic importance of the process, still today proper interpretation of the experimental data on alkane cracking remains not fully unraveled. The overall reaction sequence of alkane cracking on solid acid catalysts consists of an adsorption step, followed by the cracking steps. The mechanism of alkane cracking has received considerable attention. ${ }^{344-346}$ Catalytic cracking on monofunctional acidic catalysts may occur by two mechanisms, the classical bimolecular cracking via carbenium ions and the non-classical monomolecular cracking via carbonium ions, which was proposed by Haag and Dessau to enable rationalization of product selectivities. ${ }^{347}$ Both mechanisms may be operative : at low alkane conversion and small pore zeolites, the monomolecular mechanism prevails, whereas at high alkane conversion, alkanes preferentially react with the BAS and the bimolecular mechanism becomes predominant.

Experimentally it was observed that the apparent cracking rate increases exponentially with the number of carbon atoms in the alkane chain length. ${ }^{348,349}$ The alkane cracking activity increases with the heat of adsorption which increases monotonically with the carbon atom [vide supra]. Furthermore other structure activity relations were proposed that rely on the dimensions of the zeolite pores compared to the alkane diameter. ${ }^{350}$ When the apparent activation energies were 
corrected for the heats of adsorption, it was concluded that the intrinsic activation energies are similar for all zeolites studies ${ }^{351,352}$, a conclusion that has been questioned recently by Bhan et al. and Iglesia et al. ${ }^{353,354}$.

Previous concise summary of some literature data shows that despite the considerable attention that alkane cracking received, there are still various open questions. In such cases, theory should ideally be able to determine structure-activity relationships and to enable a proper understanding of the product selectivities. However also from a theoretical point of view, the study of alkane cracking is very challenging, as is obvious from the rich literature that is available in this area. Earlier studies primarily used small cluster models to represent the active site. ${ }^{355-359} \mathrm{It}$ is well known that the intrinsic barriers are not well described in a cluster which is too small as the confinement effects are not well described. ${ }^{299}$ More sophisticated embedded cluster models have been adopted which succeed in reproducing the barriers with sufficient accuracy. ${ }^{322,360-362}$ More and more calculations have adopted periodic boundary conditions, which are reliable as long as the adsorbate molecule is small compared to the simulation cell, to prevent unphysical interactions with neighboring cells. ${ }^{363-}$ ${ }^{366}$ Most of those simulations have adopted DFT methods as they come at a favorable computational cost. Although for a proper description of adsorption and reactions in zeolites, also dispersion effects need to be accounted for properly. Various methods have been developed as were highlighted in previous section.

To obtain reliable structure-activity relations and product distributions for alkane cracking, various effects have to be taken into account properly. Recently some very interesting studies appeared which use an innovative combination of various simulation techniques. They go beyond a static $\mathrm{OK}$ description of the reaction and rely on more extensive sampling techniques. We will highlight some of their findings hereafter. Some interesting reviews appeared also recently on multiscale modeling of hydrocarbon conversion processes, which might be interesting for more in depth reading. ${ }^{367,368}$ Swisher et al. performed a theoretical simulation of $n$-alkane cracking in zeolites using an innovative combination of various theoretical methods. ${ }^{369}$ The apparent rate constants for cracking of C3-C6 species were determined by separating the intrinsic kinetics of the alkane cracking from the thermodynamics of the adsorption step. The thermodynamics of the adsorption step was calculated using Monte Carlo simulations of the alkanes in a purely siliceous framework. The Monte Carlo simulations were also used to determine the location of the specific $\mathrm{C}-\mathrm{C}$ bonds close to the BAS. The intrinsic rate coefficients for the alkane cracking steps were determined using DFT, but on smaller clusters. The authors find that apparent and intrinsic rate coefficients for alkane cracking on MFI are systematically higher than on FAU under identical conditions. This qualitative finding agrees with recent experimental data but quantitatively their apparent rates are systematically a factor varying 
between 10 and 100 lower than the experimental values. The authors ascribe this discrepancy to the usage of the small cluster for the calculation of the intrinsic rate coefficients. Indeed they used a cluster size with $5 \mathrm{~T}$ atoms which is too small to obtain reliable values for the kinetic data. This was also clearly shown by Svelle et al. on the methylation of olefins in H-ZSM-5 . ${ }^{299}$ Cluster based calculations may give reliable kinetic data, provided that the cluster is large enough to account properly for the topology of the material. Some of the presenting authors showed a very efficient ONIOM based scheme, which was able to predict reaction rates with chemical accuracy for the same methylations studied by Svelle et al. Table 6.2.4 shows the values obtained by Swisher et al. ${ }^{369}$ and confirms that clusters of a size limited to a few number of T-sites give an overestimation of the barriers with values of about $30 \mathrm{~kJ} \mathrm{~mol}^{-1}$.

Table 6.2.4: Apparent activation energies for propane and hexane cracking on $\mathrm{MFI}^{\mathrm{a}}$.

a Experimental data taken from [1] ref. Xu et al. [2] ref. ${ }^{352}$ [3] ${ }^{351}$ [4] ref. ${ }^{348}$. The experimental values for MFI from van Bokhoven et al. ${ }^{352}$ were taken as an average over samples with different Si/Al ratios.

\begin{tabular}{lccl} 
Eact, app $\left(\mathbf{~ k J ~} \mathbf{~ m o l}^{-\mathbf{1}}\right)$ & \multicolumn{2}{c}{ calculated values } & \multicolumn{1}{c}{ observed values } \\
& 5T cluster & 23T cluster & \\
\hline propane & 179 & 148 & $147^{[1]}, 155^{[4]}$ \\
butane & 152 & 138 & $135^{[4]}$ \\
pentane & 138 & & $120^{[4]}$ \\
hexane & 127 & & $111 \pm 9.6^{[2]}, 149^{[3]}, 105^{[4]}$ \\
\hline
\end{tabular}

The comparison between the theoretically determined values and the experimental data is shown in figure 6.2.11. The qualitative picture in terms of the carbon number is well reproduced, it is indeed confirmed that for larger clusters (23T) the results are in better agreement with experimental apparent rate coefficients. The study by Swischer et al. is an interesting contribution as it combines Monte Carlo based simulations for the adsorption with quantum mechanical based simulations for the description of the reaction kinetics. 


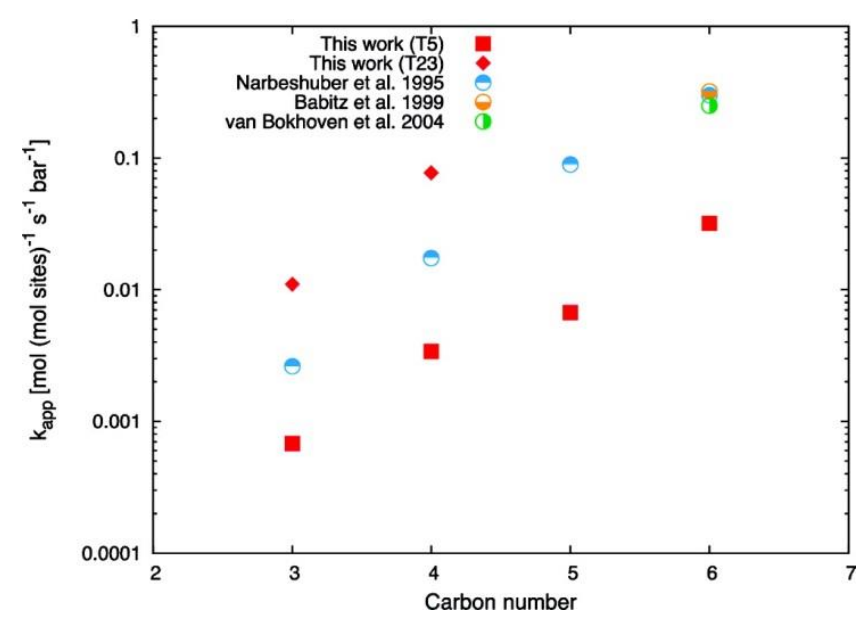

Figure 6.2.11: Comparison of calculated and experimental values of $k_{a p p}$ for the cracking of $n$-alkanes on MFI at $773 \mathrm{~K}$. Experimental data taken from van Bokhoven et al. ${ }^{352}$, Babitz et al. ${ }^{351}$ and Narbeshuber et al. ${ }^{348}$.

In another study by Tranca et al. the concept of combining Monte Carlo based simulations for the description of the adsorption are combined with DFT calculations for the intrinsic kinetics. ${ }^{310}$ The definition of the apparent and intrinsic barriers for alkane cracking is shown in Figure 6.2.12.

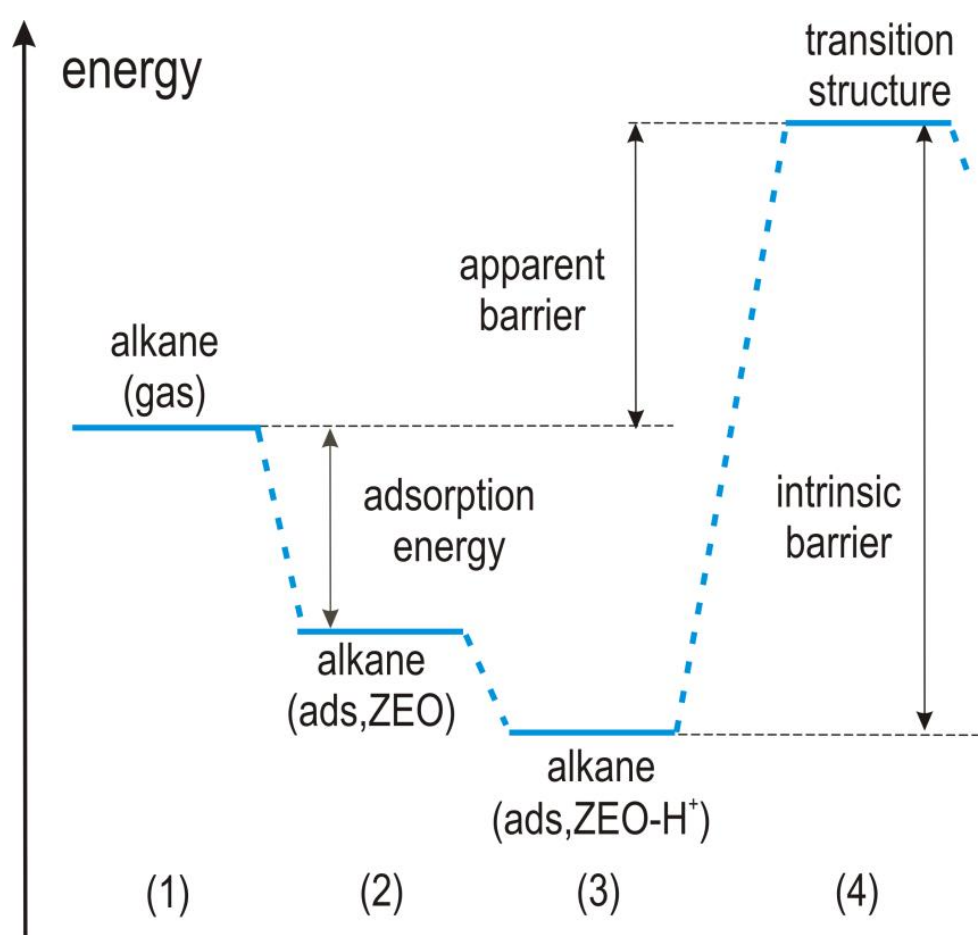

Figure 6.2.12 : Energy diagram for the formation of the first transition state in zeolite-catalyzed monomolecular cracking of alkanes: (1) Reactant in the gas phase. (2) Adsorption of the alkane in the zeolite channels. (3) Adsorption on the Brønsted site. (4) Formation of the transition state. The quantity measured experimentally is the apparent barrier, to which the appropriate energy of adsorption has to be added to get the intrinsic barrier. 
In their study the Monte Carlo simulations were used to understand the measured apparent entropy of activation in terms of the adsorption step and intrinsic reaction. Their results show that the entropy of adsorption becomes less negative with increasing temperature. Previous studies reveal that for systems where there is no strong specific adsorption with the BAS as is the case for alkane cracking, more advanced sampling methods need to be used which allow to include finite temperature effects to obtain a reliable molecular level understanding of the cracking process. Another related study was performed by Sautet and co-workers recently, where they also performed ab initio MD simulations to study the adsorption of methane, ethane, and propane in SSZ-13 at various temperatures. In accordance with previous studies, they found a dependence on the probability for finding the alkane close to the active site. They however also developed a temperature dependent expression for the active site coverages, which depends only on the chemisorption strength to the active site and not on the interaction with the zeolite wall. ${ }^{370}$ Another very challenging topic for theory is the correct prediction of product selectivities. Recently Zimmerman and co-workers showed that simulation of non-equilibrium reactive pathways is necessary to obtain reliable product distributions. ${ }^{371}$ Their study focused on $n$-pentane cracking in $\mathrm{H}-\mathrm{MFI}$. Along the pathway for $\mathrm{n}$-pentane cracking metastable intermediates, i.e. carbocations, occur which are local minima on the potential energy surface but which can easily transform in more stable species, which determine the product stabilities. Most computational studies on alkane cracking rely on the identification of one or more transition states and determine the associated activation energies. In such studies it is assumed that each transition state leads to a unique reactant and product state. In such an approach the following cases are not taken into account : (i) that multiple products may be formed from one single transition state (ii) that a given product might be the result from various transition states (iii) that various intermediates exist along the reactive pathway connecting reactants and products. Furthermore the transition states are localized by searching the $\mathrm{OK}$ potential energy surface whereas the reactive paths may be quite different at higher temperatures. To rectify some of these shortcomings Bucko and co-workers studied the cracking of propane on $\mathrm{H}-\mathrm{CHA}$ and found indeed evidence that the conditions (i) and (ii) stated above are not correct at operating conditions. ${ }^{372,373}$ Their approach relies on the transition path sampling techniques, which we will discuss further in this section. ${ }^{374}$ The approach followed by Zimmerman et al. to obtain more reliable product distribution, relies on quasiclassical trajectory simulations. ${ }^{375}$ During a QCT simulation MD simulations are performed which start from a transition state but for which the initial velocities of the atoms are populated using information about the quantum mechanical vibrational populations of the transition state. Such simulations are particularly important for cases where the zero point vibrational energy is large 
or where $\mathrm{C}-\mathrm{H}$ bonds are formed or broken during the reaction. The advantage of the approach is that reactive pathways are determined at the actual operating temperatures. In the study performed by Zimmerman et al. a QM/MM cluster approach is adopted consisting of $276 \mathrm{~T}$ atoms. The QM/MM approach was developed by Zimmerman, Head-Gordon and Bell ${ }^{360}$ and relies on a QM region that is polarized by the MM region. Recently Head-Gordon and co-workers further validated their approach on the cracking of butane in $\mathrm{H}-\mathrm{MFI}$ and found very good correspondence with experimental data. They furthermore assessed the influence of the position of the acid site on the activation barriers. ${ }^{376}$

QCT simulations give insight into the full spectrum of possible products that might be formed. Some representative snapshots along some trajectories are shown in Figure 6.2.13. The upper trajectory corresponds to ethoxide formation and propane formation, the middle trajectory yields propene and ethane and the bottom trajectory gives methane and 1-butene. The three trajectories start from a single rate limiting transition state in which the acidic hydrogen atom is shared by the $\mathrm{C} 2-\mathrm{C} 3$ fragments, yet in all three simulations the transition state evolves in other intermediates and other products. The time scale for these rearrangements is short (less than 1ps) which indicates that the metastable state does not have the time to thermally equilibrate and thus same products may be obtained from many trajectories starting from a variety of transition states. The authors succeeded in predicting qualitatively reliable product distributions. The ratio of $\mathrm{C} 3 / \mathrm{C} 2$ species is considerably better predicted using the QCT approach compared to an approach relying on information obtained from the $\mathrm{OK}$ potential energy surface. The exact ratio might still be improved if free energy calculations were performed beyond the harmonic oscillator approximation for various reactive pathways. Such calculations are nowadays possible but are still computationally expensive ${ }^{377-379}$. For selected applications in zeolite catalysis such calculations were performed by Hafner and coworkers ${ }^{372}$.

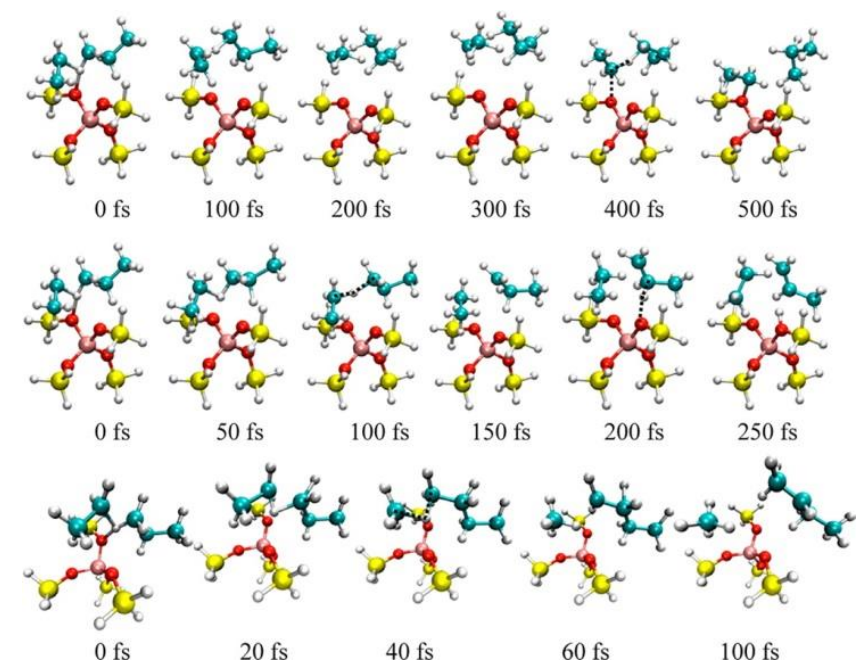


Figure 6.2.13 : Snapshots of trajectories from TS2-3B. Top: ethoxide formation. Middle: propene formation. Bottom: methane formation. Ethoxide and propene formation are common, but only one trajectory led to methane formation. Reprinted with permission from ref. ${ }^{371}$.

As already mentioned Bucko et al. adopted another approach to obtain a reliable prediction of propane cracking in $\mathrm{H}-\mathrm{CHA}$ at operating conditions, which goes beyond the harmonic oscillator approximation and goes beyond an OK potential energy description. ${ }^{372}$ They performed ab initio MD simulations for the description of the adsorbed species at finite temperature and used transition path sampling to identify the reactive paths. The final estimations of the free energies of the reaction were obtained using free-energy gradient integrations. All the simulations were performed using a periodic DFT description including dispersion interactions. Chemical transformations are rare events as their probability of occurring is very low, although once it occurs the event may be followed during a regular MD run of a few $p s$. To enhance sampling of interesting regions of the free energy surface a multitude of methods has been developed. ${ }^{54}$ Transition path sampling (TPS) is another method to study rare events and relies on creating reactive trajectories by performing trial moves starting from a point on the free energy surface. ${ }^{380}$ The TPS technique generates reactive pathways in an unbiased way and is thus conceptually very attractive. It however also comes at an expensive computational cost and can't be regarded as the method of choice for daily applications to date.

A first interesting observation resulting from the ab initio MD studies performed by Bucko et al. show that the alkane moves rather freely in the pores of the zeolite at elevated temperatures and thus adsorption cannot simply be derived from considering one point on the potential energy surface at $\mathrm{OK}$ which is subsequently corrected for finite temperature effects using the harmonic oscillator approximation. These results are in agreement with the Monte Carlo based simulations performed by Swisher et al. and also show that the entropy of adsorption is temperature dependent, which was also found by Tranca et al. ${ }^{310}$. The overall result is that the adsorption enthalpy becomes less negative and in closer agreement with the experimental values. From the TPS simulations various pathways could also be deduced corresponding to other products formed. They did not calculate explicit product distributions which are comparable to experimental data, a task which would be computationally very expensive using the TPS approach in which other competitive pathways should also be considered. They were able to assess the detailed effects of entropy along the reactive pathway. 


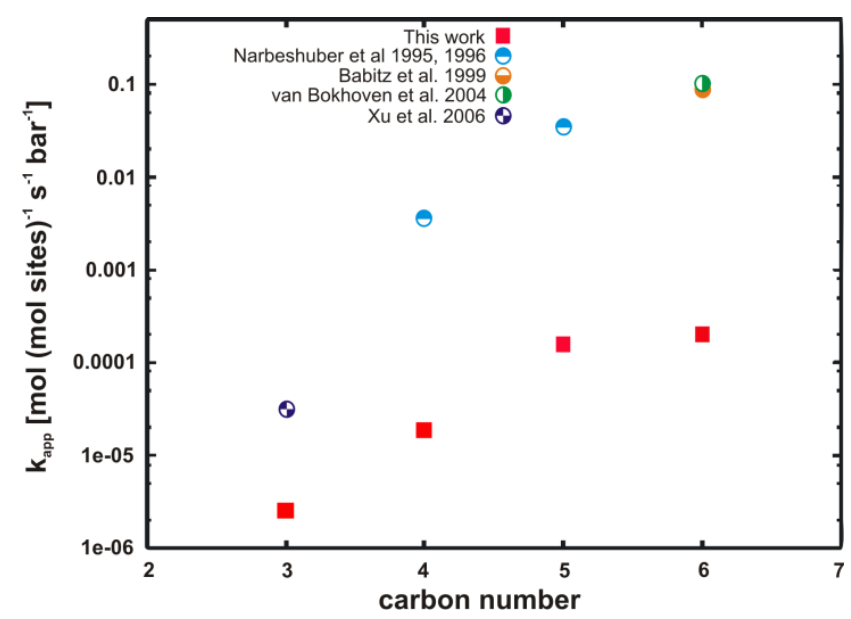

Figure 6.2.14 : Comparison of the calculated and experimental values of $k_{\text {app }}$ for the cracking of $n$ alkanes on FAU at $773 \mathrm{~K}$. Experimental data taken from van Bokhoven et al. ${ }^{352}$, Babitz et al. ${ }^{351}$, Narbeshuber et al. ${ }^{348}$ and Xu et al. ${ }^{381}$.

\subsubsection{Designing and unraveling complex reaction routes within zeolite catalysis: The methanol to hydrocarbon process}

The methanol-to-olefin process is a nice example to illustrate the importance and added value of theoretical calculations in unraveling complex catalytic cycles. The ability of H-ZSM-5 to convert methanol to hydrocarbons (MTH) in the range C2-C10 was accidentally discovered in 1976 by Mobil researchers in the search for new production routes towards high-octane gasoline. ${ }^{382,383}$ The process itself received a lot of attention both from academia and industry in the last decades, as it is one of the most prominent technologies to bypass crude oil for the production of olefins. The Methanol to Olefin (MTO) process has experimentally been developed in the past 3-4 decades and is currently being industrialized. ${ }^{384}$ Starting from a methanol source, light olefins are produced such as ethene, propene but also some medium weight hydrocarbons $(C 4, C 5, C 6$ olefins and heavier compounds) (see scheme in Figure 6.2.15). Methanol can be obtained from coal, natural gas by the production of synthesis gas which is then further processed to produce methanol. Currently the MTO process is often combined with an olefin cracking process (OCP) unit to further crack the higher fraction hydrocarbons to C2 and C3 species. ${ }^{384,385}$ Such yield improvements of ethene and propene significantly improve the return of the MTO process. From industrial point of view both the conversion of methanol to olefins and the cracking of olefins are thus very timely.

The MTO chemistry mechanism is probably one of the most disputed processes within heterogeneous catalysis from mechanistic point of view. ${ }^{386,387}$ For more than 30 years it was believed that two $\mathrm{C} 1$ species couple directly to form the first carbon-carbon bond. Currently there is a consensus that a hydrocarbon pool mechanism operates in which an organic center is trapped in 
the zeolite pores and acts as co-catalyst, as schematically shown in Figure 6.2.15. ${ }^{388-393}$ In very general terms, the mechanism consists of successive methylations of the hydrocarbon pool species by a methylating agent, such as methanol or dimethylether, after which light olefins are split off.

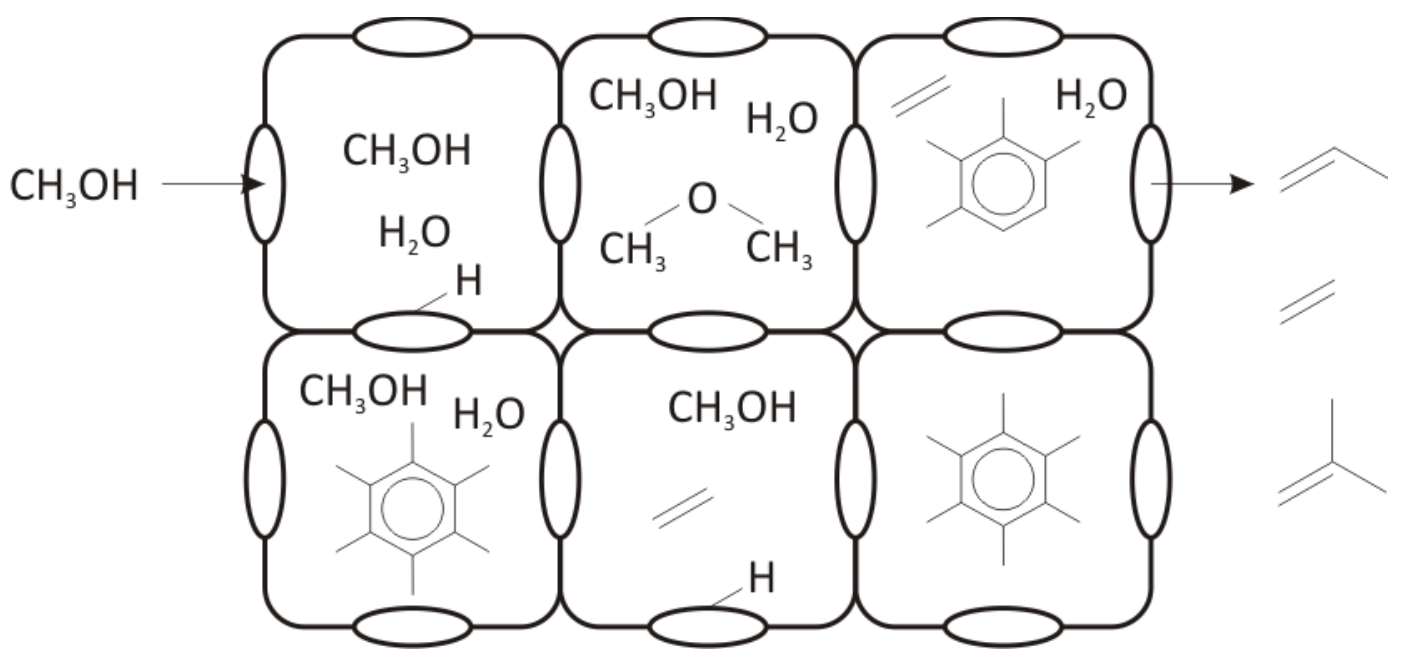

Figure 6.2.15 : Schematic representation of possible compositions of zeolite pores during the MTO process.

The hydrocarbon pool may be of aromatic type but also alkenes can take up this role. In some materials a dual cycle operates depending on the operating conditions (Figure 6.2.16). In that case alkenes themselves may be successively methylated after which by cracking lower olefins are produced. Both cycles are intertwined and the importance of various reaction steps, intermediates and reaction cycles critically depends on a multitude of factors such as catalyst topology, operating conditions and catalyst composition. Various aspects of the methanol to hydrocarbon process have been recently reviewed by Olsbye and co-workers ${ }^{384}$.

From a modeling perspective the MTO process is very challenging as it involves a very complex process, for which proper account of various factors need to be taken into account such as longrange non-bonding interactions, zeolite topology, influence of surrounding molecules such as methanol and water. In addition to all these factors, it is important to emphasize that the zeolite catalyst has an inherent dynamical behavior, i.e. the host framework is to some extent flexible and may promote or inhibit some reaction routes. It is not our intention to give a complete review of all theoretical aspects related to the MTO process as some of the present authors have recently published two reviews on both the mechanistic aspects of the MTO process and the methods to obtain accurate kinetic data for individual reaction steps. ${ }^{279,} 394$ Instead we will focus on some recent theoretical contributions which used promising theoretical methods that allow the simulation of the process at operating conditions. 


\section{Relevant simulations of some MTO reactions at operating conditions.}

In all proposed reaction cycles of the MTO process, methylation reactions play a prominent role and have thus far been the subject of numerous experimental and theoretical studies. ${ }^{152,}$, 275, 299, 395-399 However to date the question remains whether the prevailing mechanism is either the stepwise or concerted mechanism. In the concerted mechanism the methylation occurs in one single step via an $\mathrm{SN}_{2}$-like mechanism, whereas in the stepwise mechanism first framework-bound methoxide species are formed which can then further take the role as methylating agent. Many theoretical studies took the concerted mechanism into consideration and very good agreement between theoretical and experimental kinetic data was obtained. ${ }^{275,299}$ In fact previous references have shown that it is possible to obtain chemical kinetics with near chemical accuracy provided that the reaction takes place at a well-defined active site and assuming the concerted mechanism. To resolve the issue about the prevailing mechanism, one needs to account however for the process conditions such as methanol pressure, temperature,....

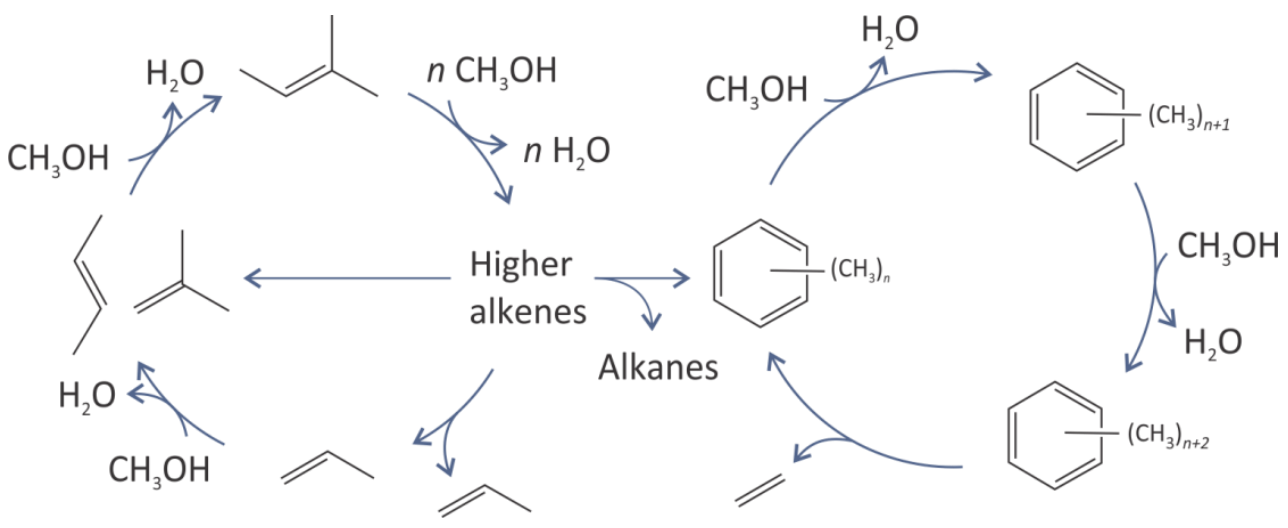

Figure 6.2.16 : Suggested dual-cycle concept for the conversion of methanol over H-ZSM-5. Reprinted with permission of ref. ${ }^{384}$.

Recently Brogaard et al. performed a combined theoretical and experimental approach to study the various methylation pathways in ZSM-22. ${ }^{400}$ For the theoretical modelling they combined DFT calculations of free energy profiles with micro-kinetic modelling of the competitive pathways between various alkenes and methanol. The combined approach reveals that alkene methylation proceeds via the stepwise mechanism at temperatures of MTH conversion. A similar conclusion was recently made by Jones and Iglesia using a kinetic, spectroscopic and theoretical study ${ }^{401}$, showing that methoxy-mediated dissociative routes become prevalent at higher temperatures and lower pressures due to a delicate balance between entropic and enthalpic effects. This subtle interplay is further explained using Figure 6.2.17, which is based on the results presented by Van Der Mynsbrugge et al. ${ }^{313,402}$. The results show that all intrinsic free enthalpy barriers for all methylation 
steps are relatively similar. However when the free energy barriers are further separated into their enthalpic and entropic barriers, it becomes clear that the methoxide formation step involves a lower entropic barrier, compared to the direct methylation step. This further gives evidence that the stepwise mechanism would be preferred at higher temperatures, due to favourable entropic effects. Given this discussion it becomes clear that disclosure of various competitive pathways may certainly not solely be made on basis of OK energy profiles as was often done in older literature. More indepth information on the interplay between enthalpic and entropic effects may be found in the work of Gounder and Iglesia ${ }^{403}$. Further relevant examples within the context of the MTO process and the role of entropy and enthalpy to investigate the influence of topology on the reaction kinetics may be found in reference ${ }^{404}$.

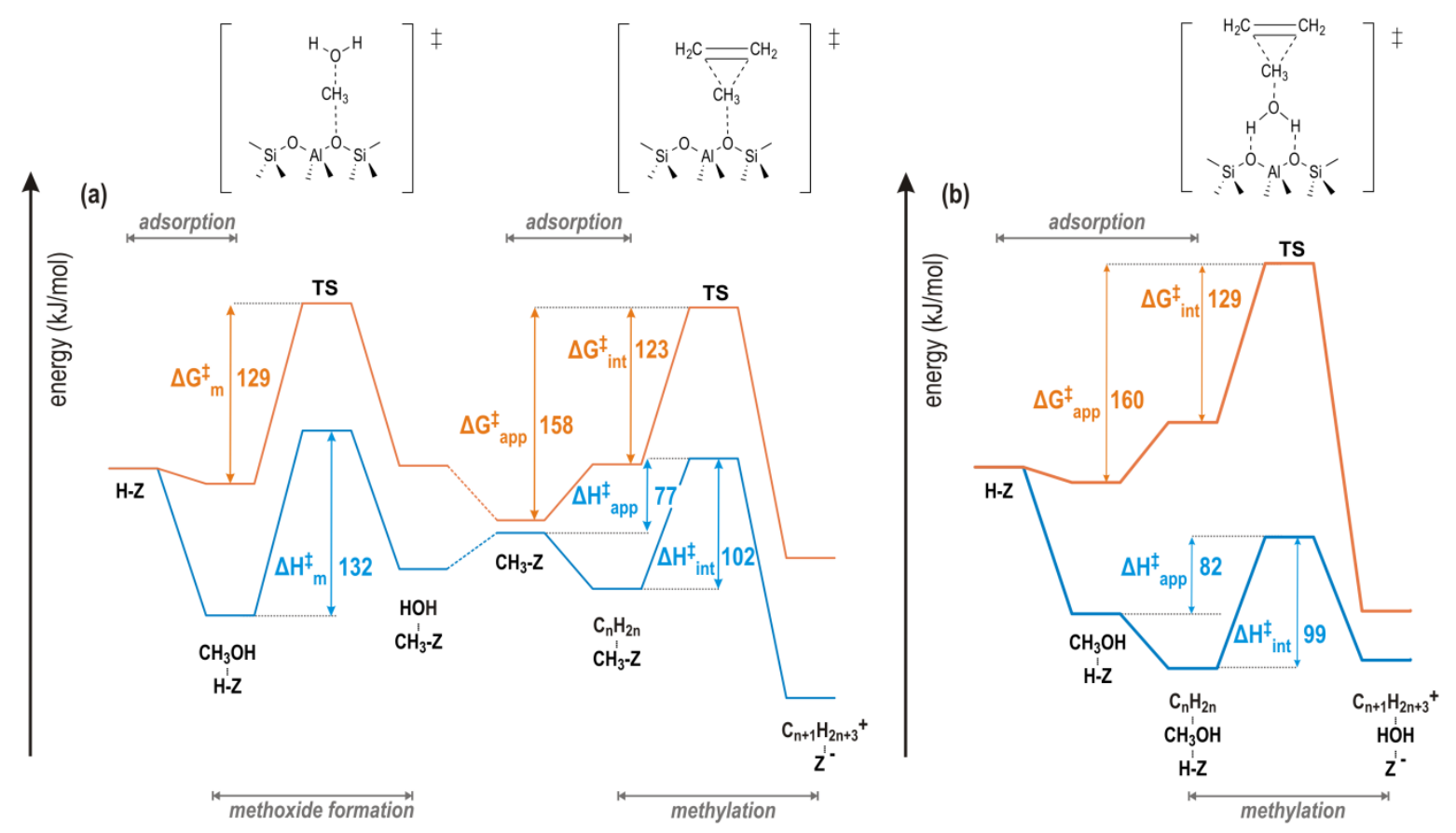

Figure 6.2.17 : Schematic enthalpy (blue) and free-energy (orange) profiles for alkene methylation.

a) Stepwise mechanism: after formation of a framework-bound methoxide (from methanol or dimethyl ether, with or without assisting molecules), the alkene co-adsorbs onto the methoxide and is subsequently methylated. b) Concerted mechanism: the alkene co-adsorbs onto a hydrogenbonded methanol or dimethyl ether and is methylated in a single step. All energies in $\mathrm{kJ} \mathrm{mol}^{-1}$. Adapted from ref. ${ }^{313}$.

To study the influence of the strength of the acid sites, Wang et al. recently proposed a reactivity descriptor model. They showed that the ammonia heat of adsorption correlates very well with the energy barrier of transition states observed in the methylation of propene in various zeotype 
catalysts. Such reactivity descriptor models may grow in importance in combination with microkinetic modelling. ${ }^{405}$

An important effect which needs to be accounted for when modelling the MTO process is the influence of additional guest molecules in the pores of the material. Indeed industrial MTO processes typically operate at high methanol partial pressures. Furthermore, water is present in the feed and sometimes also added on purpose to tune the product selectivity. Most theoretical studies have neglected the influence of these additional guest molecules. Indeed simulations of such effects require an approach that goes beyond a standard methodology, which is based on a limited number of points on the potential energy surface. Instead extensive sampling of the free energy surface (FES) is required, to account properly for entropic effects and for various minima on the FES. Only a limited number of studies have applied techniques that go beyond the static approach to describe chemical transformation in zeolites. ${ }^{371,372,406-410}$ Earlier spectroscopic and theoretical studies reported proton transfer and mobility in zeolites, depending on the loading of protic molecules like methanol and water in the pores. ${ }^{52,411-414}$ Recently Moors et al. performed an extensive study on the methylation of benzene in $\mathrm{H}-\mathrm{ZSM}-5$ in presence of various guest molecules such as methanol,... ${ }^{415}$ A metadynamics approach was adopted as developed by Laio and Parrinello, to sample also chemical reactions with reasonable accuracy within the time frame of the MD simulations. ${ }^{416}$ The dynamical calculations showed that there is a reasonable probability of forming protonated clusters in the zeolite pores. It was concluded that a simple view of a localized Brønsted acid site at which the reaction occurs is far too simplistic. Studies including such effects will become increasingly important if focus is set on comparing theoretical and experimental data at operating conditions. Bell and co-workers recently demonstrated that $0 \mathrm{~K}$ potential energy surfaces alone cannot accurately predict product selectivities as selectivity in zeolite catalysis is largely determined by dynamical effects in high-temperature reaction pathways. ${ }^{371,410}$ More details on these dynamical effects were taken up in reference ${ }^{279}$.

\section{Full catalytic cycle for the production of ethene and propene in H-ZSM-5 and H-SAPO-34.}

Among various MTO catalysts, $\mathrm{H}-\mathrm{ZSM}-5$ and $\mathrm{H}-\mathrm{SAPO}-34$ are known as the archetypal MTO catalyst materials since they are both used for commercial methanol conversion. Their distinct topology differences lead to various product distributions, selectivities and deactivation processes. ${ }^{384,394}$ Despite numerous studies full catalytic cycles in both materials are not yet fully unraveled. Theory has contributed substantially towards identifying plausible reaction cycles. ${ }^{417,418}$ Hereafter we highlight some recent insights based on combined theoretical and experimental work. More in particular spectroscopy has shown to be very important for the characterization of intermediates 
during MTO conversion. For more detailed discussions we refer to numerous publications in the field.

A schematic representation of the aromatic based cycle which is operative both in $\mathrm{H}-\mathrm{ZSM}-5$ and $\mathrm{H}$ SAPO-34, is shown in Figure 6.2.18. It must be noted that other cycles may equally be operative and intertwined with this cycle, such as the alkene based cycle (vide supra). The first full working cycle within ZSM-5 was proposed by some of us in 2008 together with Haw and co-workers. Our proposal

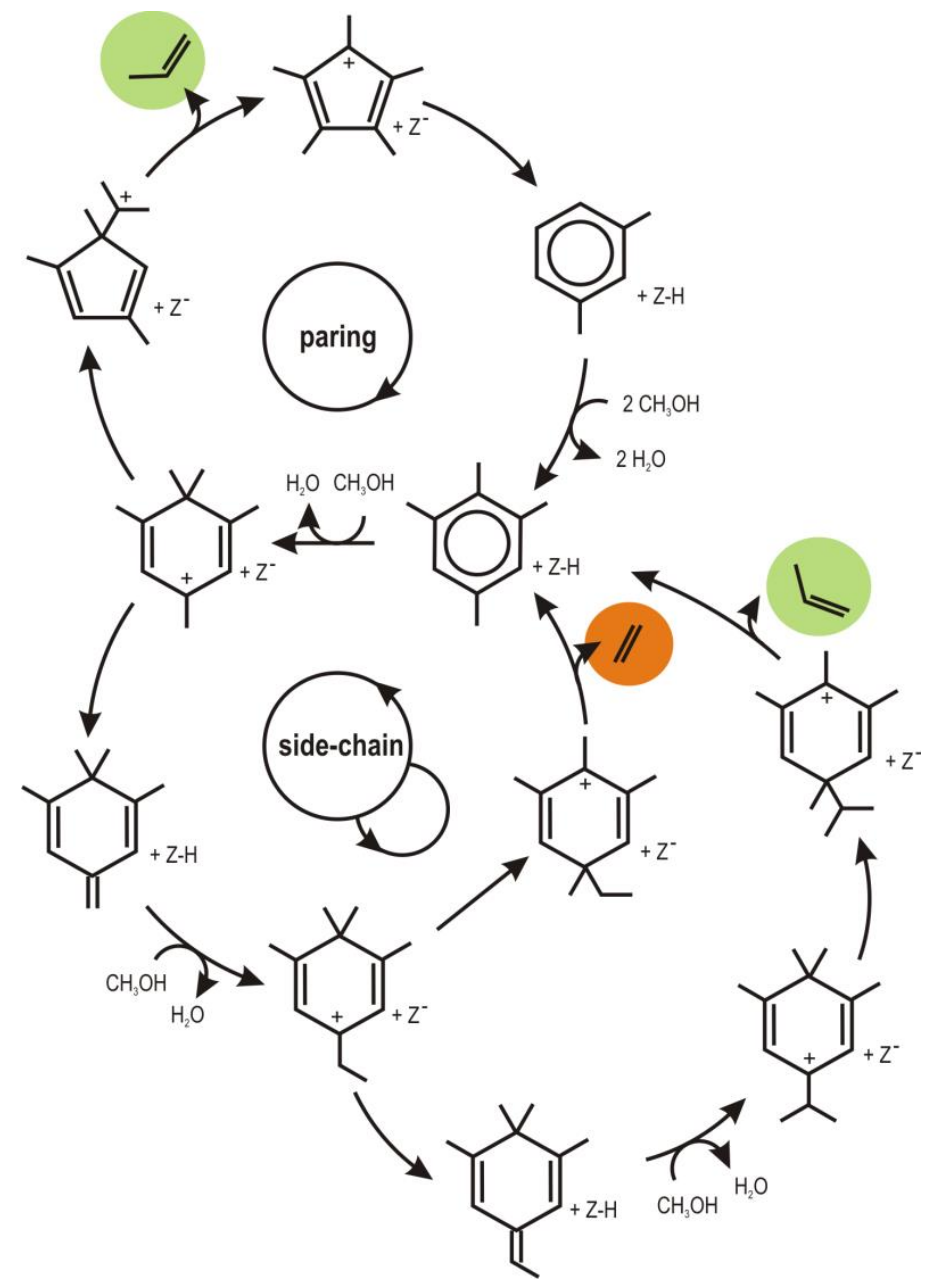

Figure 6.2.18: Overview of various hydrocarbon pool based cycles and their interaction within MTO/MTH chemistry. The specific operating cycle and products depends on the catalyst employed and on the reaction conditions. [Figures adapted from ref. ${ }^{417,419}$ ]

was based on rate constants for each individual step that were calculated using quantum chemical simulations on a supramolecular model of both the H-ZSM-5 zeolite and the co-catalytic hydrocarbon pool species. ${ }^{418}$ Furthermore the proposed cycle succeeds in tying together various experimental clues. We used the fact that some cyclic cations were experimentally observed using NMR spectroscopy (Figure 6.2.19), that scrambling of labelled methanol in the feed was observed in 
the aromatic rings and that isobutene was the third most abundant product in ZSM- 5 . This led us to propose a cycle based on the paring cycle, which was able to produce isobutene.

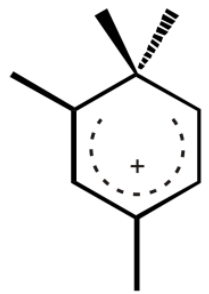

(1)

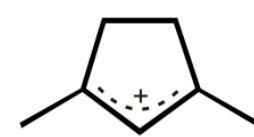

(2)

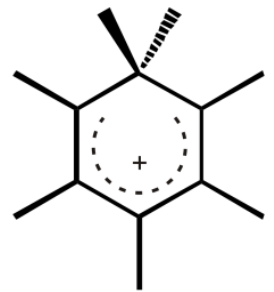

(3)

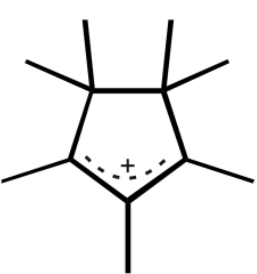

(4)

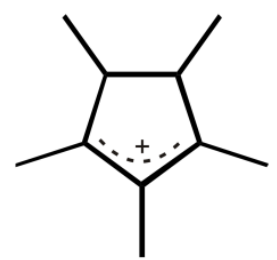

(5)

Figure 6.2.19 : Series of carbenium ions observed in zeolites: 1: 1,1,2,4,6-pentamethylbenzenium cation; 2: 1,3-dimethylcyclopentenyl cation; 3: heptamethylbenzenium cation; 4: heptamethylcyclopentenyl cation; 5: pentamethylcyclopentenyl cation.

Full catalytic cycles consisting of low-barrier elementary steps for the production of ethene and propene in ZSM-5 were to date not proposed from ab initio point of view. It is believed that propene production would be partly governed by the alkene cycle ${ }^{361,420}$ but ethene should be produced by the aromatic based cycle. Our previous work on ZSM- 5 unraveled almost all steps apart from the olefin elimination step which was still too highly activated.

Very recently we succeeded however to propose a full working cycle in H-SAPO-34 based on the side chain mechanism, that produces both ethene and propene. We proposed a new transition state for the olefin elimination step which critically depends on long range interactions in the transition state and various assisting molecules. ${ }^{417}$ The various steps are schematically given in Figure 6.2.18. The highest free energy barriers in the side-chain route proposed in H-SAPO-34 remain below $100 \mathrm{~kJ} \mathrm{~mol}^{-}$ ${ }^{1}$, which is much smaller than other barriers of the order of $200 \mathrm{~kJ} \mathrm{~mol}^{-1}$ in which more unstable structures were present. ${ }^{419,421}$ It remains to be unraveled theoretically in how far this cycle may also be operative in H-ZSM-5.

Recently some very interesting experimental spectroscopic and mechanistic studies have appeared, showing clear evidence for the paring mechanism in H-ZSM-5. ${ }^{86,87}$ Wulfers and Jentoft investigated the occurrence of hydrocarbon pool species in H-ZSM- 5 and H-beta by using in situ UV/Vis spectroscopy. They observed an absorption band at $295 \mathrm{~nm}$ on H-ZSM-5 which was assigned to an alkyl-substituted cyclopentadienium ion with four or five alkyl groups. The growth of the band could be correlated with the increase in ethene formation during an induction period. Within chabazite type materials, Xu et al. also observed recently the occurrence of the heptamethylbenzium cation and the pentamethylcyclopentenyl cation (species 3 and 5 in Figure 6.2.19) under real working conditions for MTO conversion. They used a combination of techniques such as NMR, GC-MS and 
theoretical calculations. These five membered cyclic cations points towards the occurrence of a paring like mechanism in chabazite type materials in addition to the already identified side chain cycle, although the barriers for the paring type cycle were still relatively high. ${ }^{422}$ Detailed insight into these mechanisms is of utmost importance to address the factors controlling the selectivity towards C2 and C3 species. Apart from retrieving insights into detailed mechanistic proposals to control the product selectivity, experimental studies clearly showed that various other aspects are very important to tune the product distribution such as crystal size, acidity, deactivation, temperature conditions.... Of course these aspects are strongly correlated with each other. Modeling may contribute to obtain deeper insights into some of the governing factors for the process.

An aspect that received more attention recently in the context of MTO chemistry and particularly the product distribution is the importance of diffusion of reactants and products through the pores of the material.

It has been acknowledged from various experimental studies that diffusion of products and reactant molecules is an important factor to control the selectivity. ${ }^{423-425}$ This effect is closely related to deactivation and formation of larger hydrocarbon pool species in the pores of the material. ${ }^{426}$ We will discuss some aspects related to diffusion in section 6.4.

\subsection{Redox/oxidation systems within zeolite chemistry}

\section{3.1 Hydrocarbon oxidation in microporous aluminophosphates.}

A major challenge in the field of hydrocarbon oxidation is the development of catalysts that can effect selective oxidation using molecular oxygen. Experimental work ${ }^{427-430}$ explored the use of the microporous alumino-phosphates (ALPOs) doped with transition metal ions for such catalytic processes, as unlike zeolites, ALPOs readily allow transition metal substitution at the aluminum sites. The work indeed showed that these materials could act as effective catalysts and could selective oxidize alkanes to alcohols, ketones and acids. However, the question of the mechanisms of such reactions remained open, although there was good evidence that they proceeded by a radical mechanism.

This problem was addressed by Gomez-Hortiguela and coworkers in a series of papers ${ }^{76,431-436}$, which investigated the oxidation of ethane in Mn substituted ALPO-5. Ethane was chosen for reasons of computational efficiency, although the mechanistic details which emerge from the study should be general for linear alkanes (excepting, of course, the question of regio-selectivity which is not addressed in this work). The calculations employed periodic boundary conditions with the 
CRYSTAL code (in which localised atomic centered basis sets are used) and the B3LYP exchangecorrelation functional.

The results of the calculations confirmed the radical mechanism and were able to chart a complete and detailed mechanism for this important catalytic process including activation, propagation and termination/regeneration. The $\mathrm{Mn}$ ion substituting for $\mathrm{Al}$, provides the redox active site and cycles between the two oxidation states of II and III during the oxidation reaction. Figure 6.3.1 illustrates the crucial initial activation steps: the first is the abstraction of hydrogen by a framework oxygen adjacent to the $\mathrm{Mn}(\mathrm{III})$ ion. The hydrogen atom effectively reduces the transition metal to $\mathrm{Mn}(\mathrm{II})$ and protonates the oxygen. The resulting $\mathrm{C}_{2} \mathrm{H}_{5}$ radical is loosely bound to the framework $\mathrm{OH}$ but then reacts with molecular oxygen to form a super-oxide species which binds to the $\mathrm{Mn}(\mathrm{III})$. The mechanism developed by Gomez-Hortiguela then explores the subsequent fate of this species, which via a series of reactions involving the redox site, generates the observed oxygenated products. The mechanistic scheme proposed for the propagation cycle which is illustrated in Fig 6.3.2 is amongst the most complex yet revealed by computational studies of microporous materials. A detailed discussion is beyond the scope of the present article but a useful overview is provided in reference ${ }^{431}$. Subsequent studies have investigated how the mechanism differs in Fe substituted materials. ${ }^{436}$

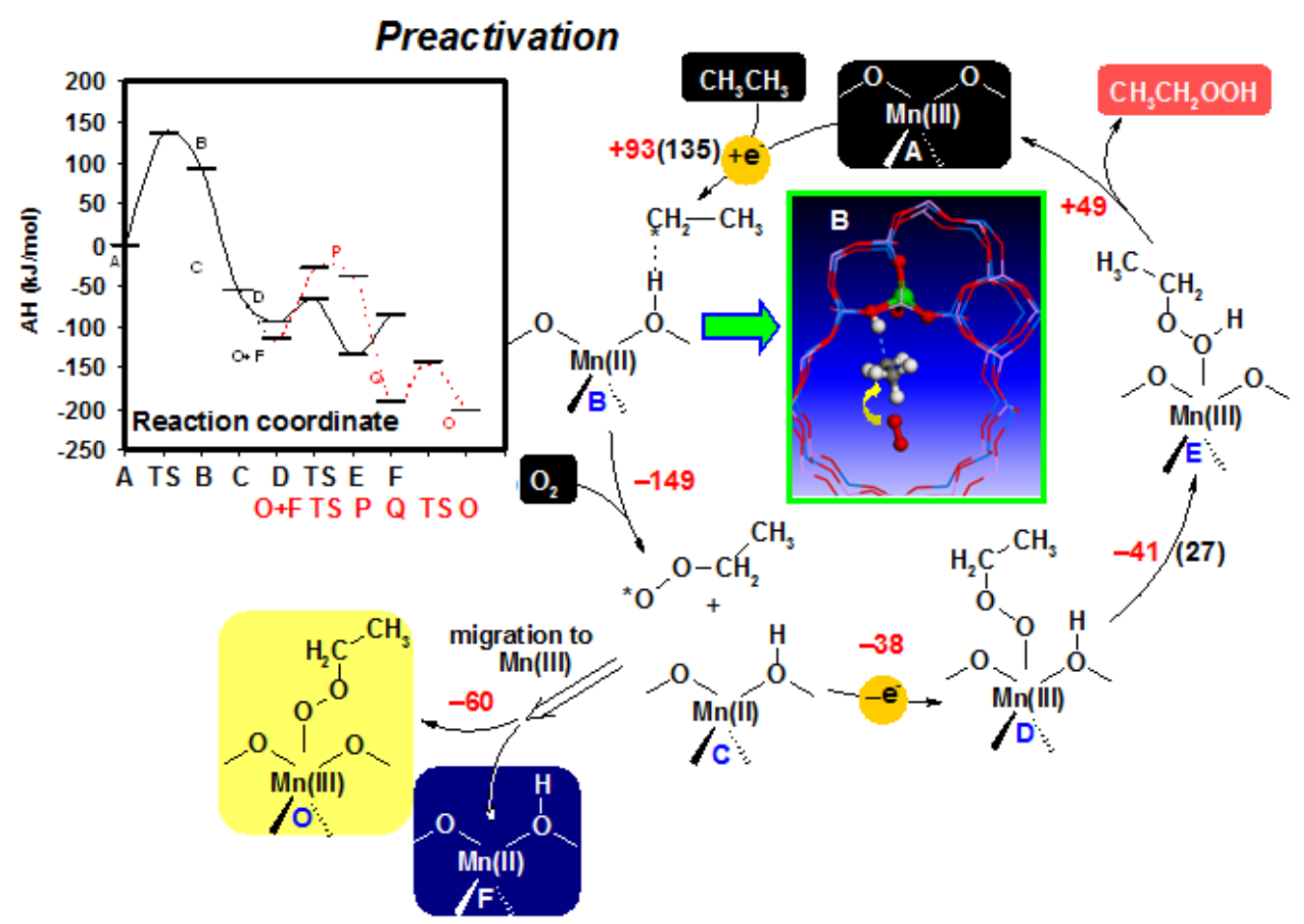


Figure 6.3.1: Preactivation mechanism for ethane oxidation in Mn-ALPO-5. A black background indicates initial catalyst and reactant molecules, while a red background indicates the hydroperoxide intermediate. Other background colors (yellow, blue) are used to indicate intermediates produced here and necessary to initiate subsequent reaction cycles. Enthalpies (red) and activation energies (black, in brackets), in $\mathrm{kJ} \mathrm{mol}^{-1}$, are shown for each elementary step . Reprinted with permission from ref. ${ }^{76}$.

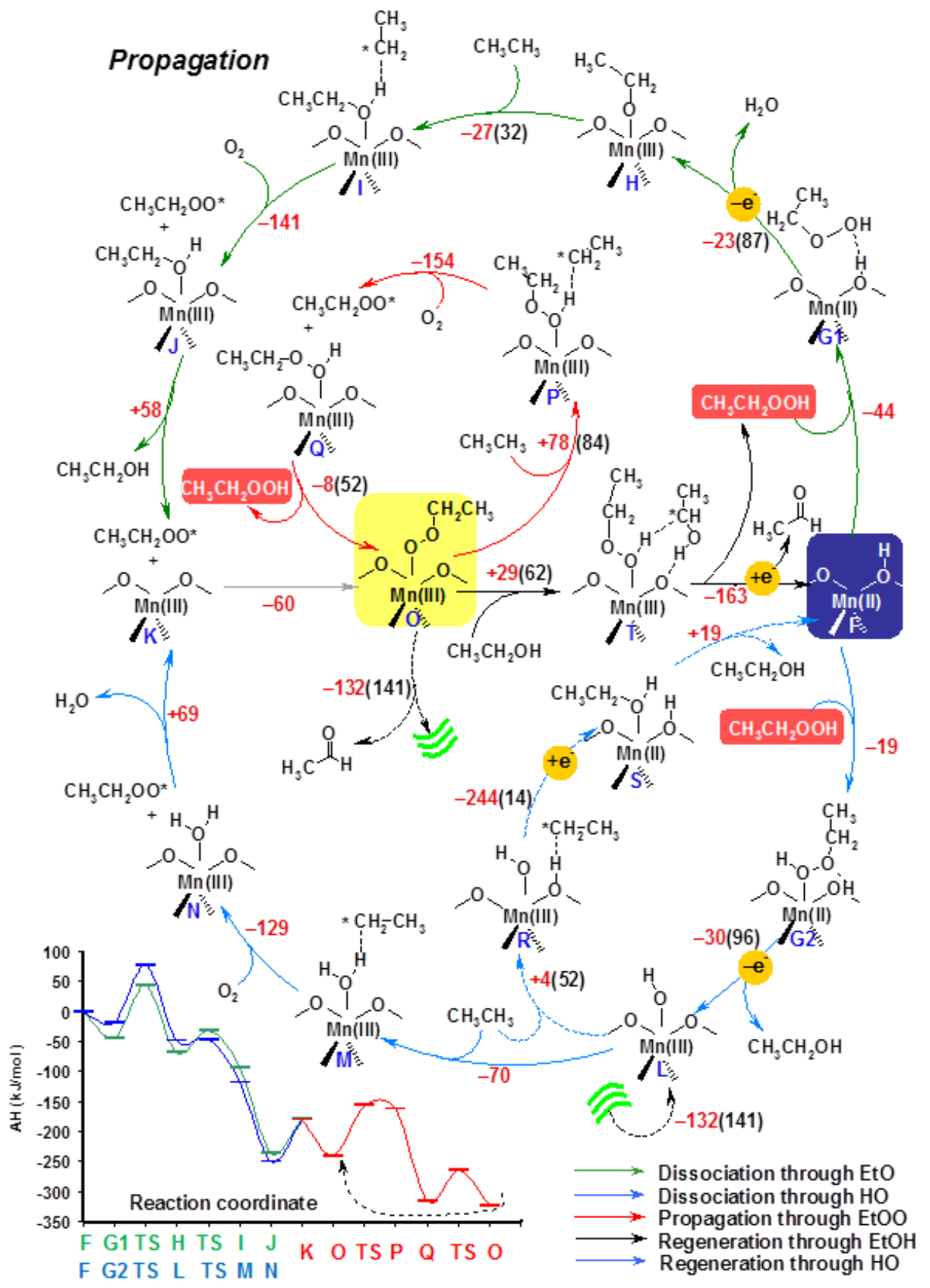

Figure 6.3.2 : Propagation mechanism for ethane oxidation in MnALPO-5. Background colors and energies are represented as in Figure 6.3.1. Reprinted with permission from ref. ${ }^{76}$. 


\subsubsection{Titanium substituted zeolites as redox catalysts}

Microporous Ti substituted zeolites have proved to be effective and versatile partial oxidation catalysts. ${ }^{437}$ The classic system is TS-1 In which a small proportion ( 1\%) of the framework Si in silicalite is substituted by $\mathrm{Ti}$, and which is used as an industrial partial oxidation catalysts. An intriguing variant on this theme involves the grafting of Ti centers on the internal walls of MCM zeolites, which have also been extensively studied. ${ }^{438,439}$ In this section, we highlight the role of modelling using cluster and embedded cluster ( $Q M / M M)$ calculations in elucidating active site structures and activation energies in these catalysts.

The first requirement is a reliable model for the structure of the substituted Ti centers. The most detailed study addressing this problems was reported by To et al ${ }^{440,441}$, who used QM/MM techniques to model Ti substitutional centers in silicalite. $\mathrm{QM} / \mathrm{MM}$ is a powerful tool in computational catalytic science as it allows the computational power to be focused on the active site and its immediate surroundings while the more distant regions of the lattice are described by more approximate models. In the case the modelling of Ti-zeolites, the titanium center and its surrounding coordinating tetrahedra are described using a quantum mechanical (DFT) method while the surrounding lattice is modelled using interatomic potential (or MM) methods. The QM cluster is terminated by hydrogen atoms to cap the terminal dangling bonds. The CHEMSHELL code ${ }^{442-444}$ was used, which is an effective and versatile tool in $\mathrm{QM} / \mathrm{MM}$ modelling. The calculations examined in detail the possible hydrolysis reactions illustrated in Fig 6.3.3.

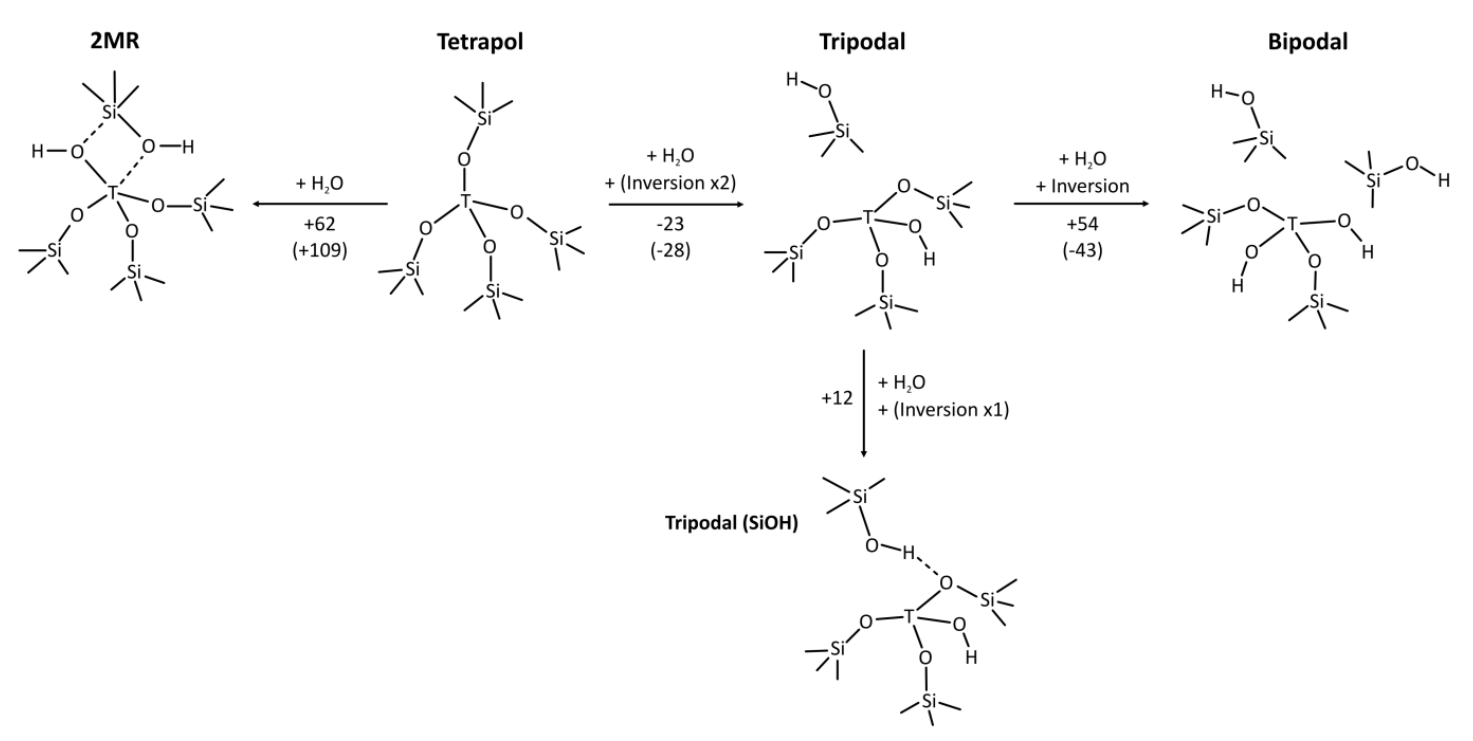


Fig. 6.3.3: Hydrolysis reaction scheme for Si and Ti sites in TS-1. Calculated formation energies in $\mathrm{kJ}$ $\mathrm{mol}^{-1}$. The energies indicated are calculated from Ti- and Si-centered clusters (Si values in parentheses).

The modelling shows that the process involving hydrolysis and inversion around the Ti sites is favorable for one water molecule resulting in a tripodal site, but that subsequent hydrolysis steps are endothermic. Both the un-hydrolyzed tetra-podal and hydrolyzed tri-podal sites are likely to play a significant role in the catalytic processes.

The next step involves coordination of the oxidant to the Ti center. Here a variety of hydroperoxide species $(\mathrm{ROOH})$ including hydrogen peroxide have been studied both computational and experimentally. Earlier work of Barker et al. using simpler un-embedded cluster calculations had identified the stability of both bidentate and mono-dentate binding configurations of the peroxy species to the Ti center as illustrated in figure 6.3.4.

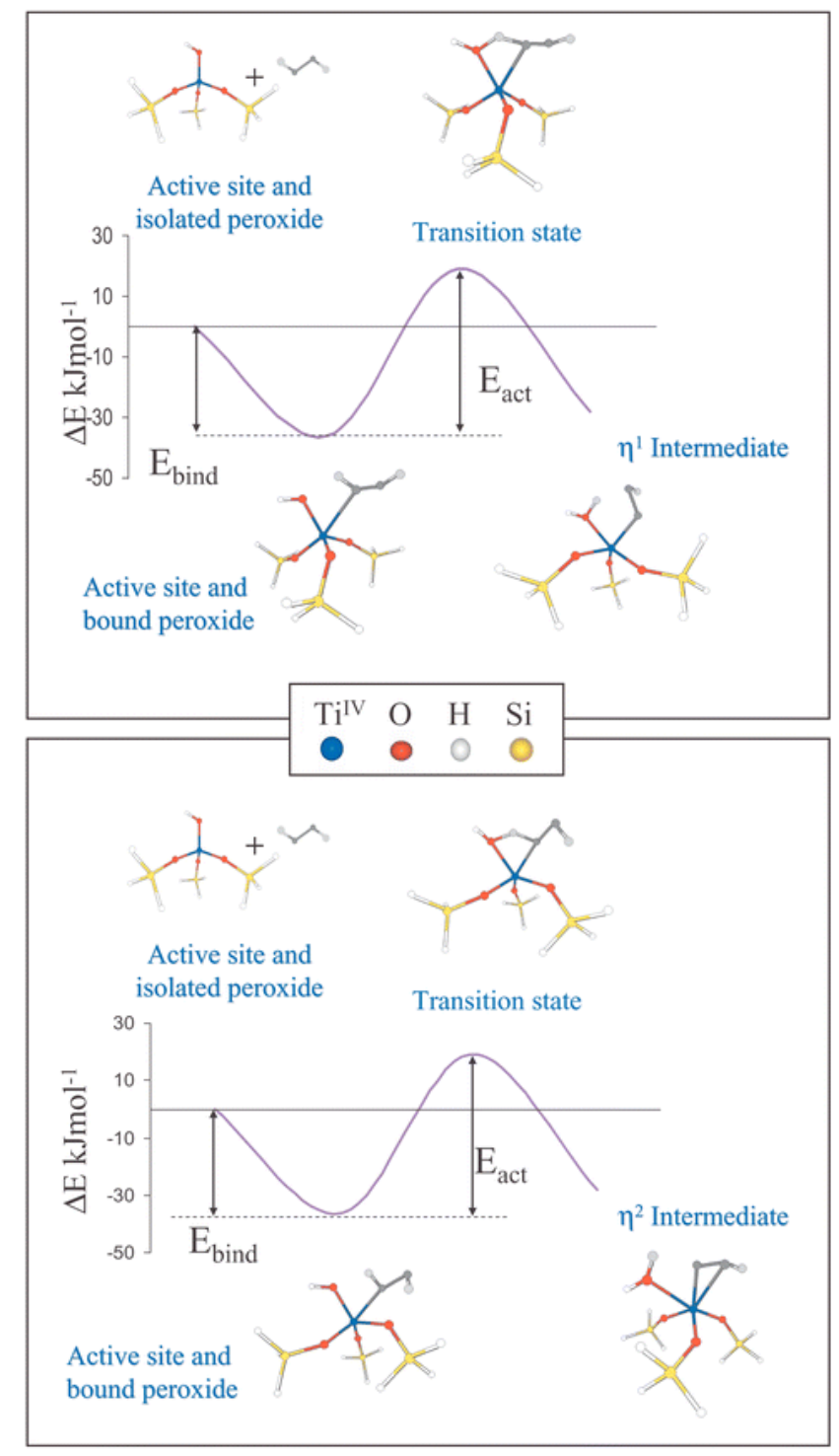


Fig 6.3.4: Two modes of coordination of $\mathrm{H}_{2} \mathrm{O}_{2}$ to $\mathrm{Ti}$ in Ti-micro/mesoporous catalysts. Approximate transition states are identified. Adapted from ref. ${ }^{445}$.

Similar configurations were identified in the more detailed $\mathrm{QM} / \mathrm{MM}$ study which also showed that the most stable structure involved coordination to the tri-podal site shown in Fig 6.3.3 and created by hydrolysis and inversion. It is also worth noting that the configurations identified by the simulations fit in well with in-situ EXAFS studies of Sankar and co-workers . ${ }^{446}$

We have now therefore by a combination of computation and experiment identified the active intermediate which effects the oxidation catalysis. The final stage is the oxidation reaction effected by the electrophilic oxygen bonded to the titanium atom to give the full catalytic cycle sketched in Fig 6.3.5 for the case of epoxidation of alkenes.

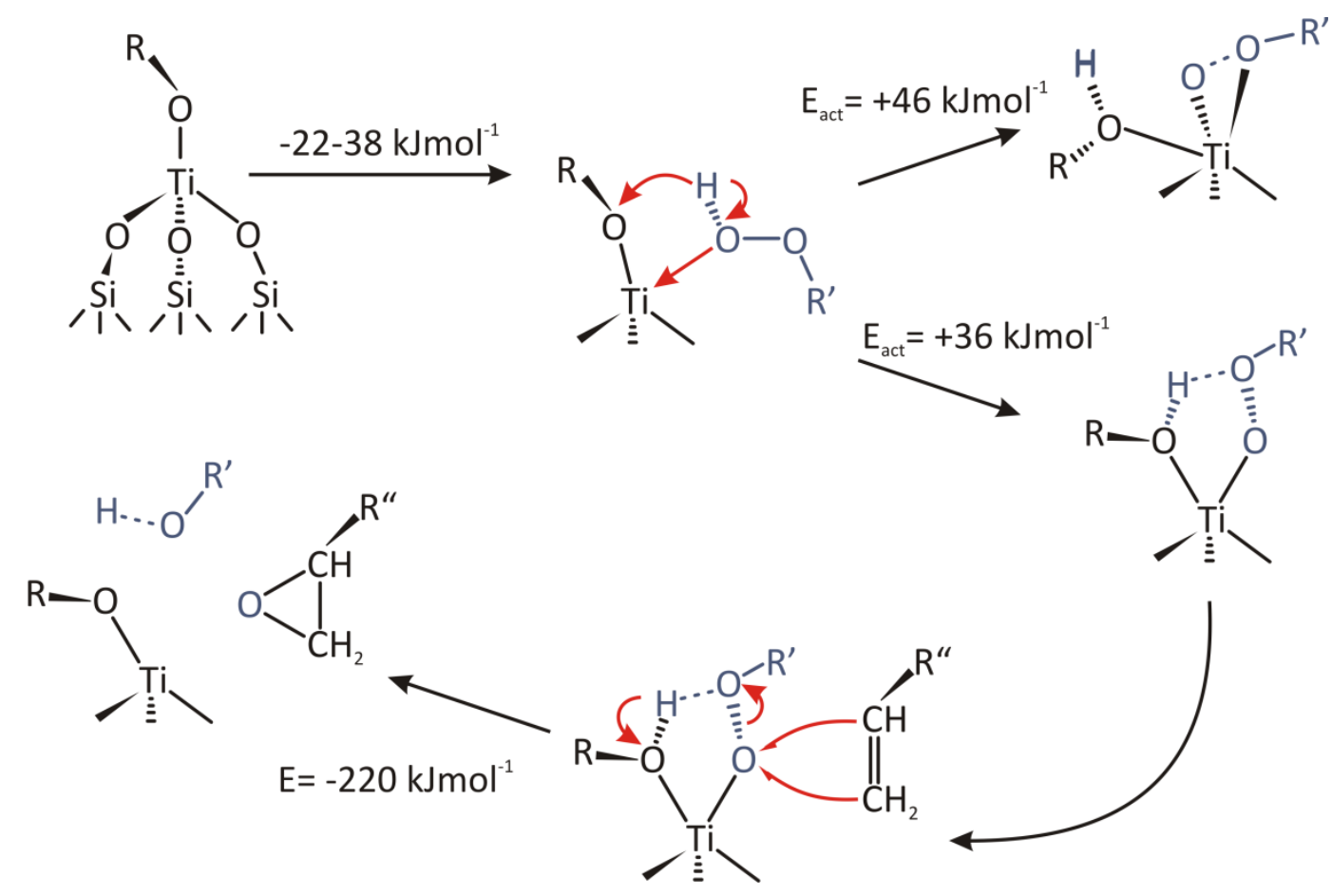

Figure 6.3.5: Proposed epoxidation mechanism for Ti micro/meso-porous catalysts for alkene epoxidation. The energies of the case $\mathrm{R}=\mathrm{H}$.

These calculations illustrate the value of this class of calculation in elucidating active site structures and mechanisms in zeolite catalysis An indication of the quantitative reliability of the methods is given by a recent study of Berger, Logsdail et al. ${ }^{447}$, which used QM/MM methods to calculate 
reduction potentials for in framework Fe in Fe-ZSM-5, that were in good agreement with experimental data.

\subsubsection{Metal-exchanged zeolites for $\mathrm{NH}_{3}-\mathrm{SCR}$ : $\mathrm{Cu}-\mathrm{SSZ}-13$ as a case study}

As a third case study, we focus on metal-exchanged small pore zeolites, and in particular Cu-SSZ-13, which lately have been adopted for use in the ammonia-assisted Selective Catalytic Reduction $\left(\mathrm{NH}_{3}-\right.$ $\mathrm{SCR}$ ) of $\mathrm{NOx}$. $\mathrm{NH}_{3}-\mathrm{SCR}$ chemistry is one of the leading $\mathrm{NO}_{x}$ emission control techniques for lean-burn diesel engines and its optimization is of significant importance to the automotive industry. The complex reaction process challenges both experimental and theoretical investigations. Recently, $\mathrm{Cu}$ SSZ-13 has attracted a lot of attention, mainly due to its improved hydrothermal stability and high $\mathrm{NH}_{3}$-SCR activity, whereas it is only moderately active in nonselective NH3 oxidation. ${ }^{448}$ The latter results from the fact that the catalytic centers are isolated $\mathrm{Cu}^{2+}$ ions ${ }^{449}$, which are intrinsically less active in oxidation reactions than $\mathrm{Cu}$ ion dimers and $\mathrm{CuO}_{x}$ clusters. In particular, main findings of $\mathrm{Cu}$ SSZ-13 as selective $\mathrm{NH}_{3}$-SCR catalyst were recently reviewed by Gao et al. ${ }^{450}$. The small unit cell of the CHA topology (depicted in Figure 6.3.6) of CU-SSZ-13 has clear computational advantages. In particular, the hexagonal unit cell contains 36T-sites with corresponding oxygen atoms. Alternatively, a minimal rhombohedral unit cell containing $12 \mathrm{~T}$-sites can also be applied. Double-six ring units (d6r) are ordered in such a way to construct spacious cages with are only accessible via relatively narrow 8-ring windows (free diameter of approximately 3.6 A). Previous studies point out that the copper ions can reside near the top of the $d 6 r$ unit or near the 8-ring windows, as also displayed in Figure 6.3.6.

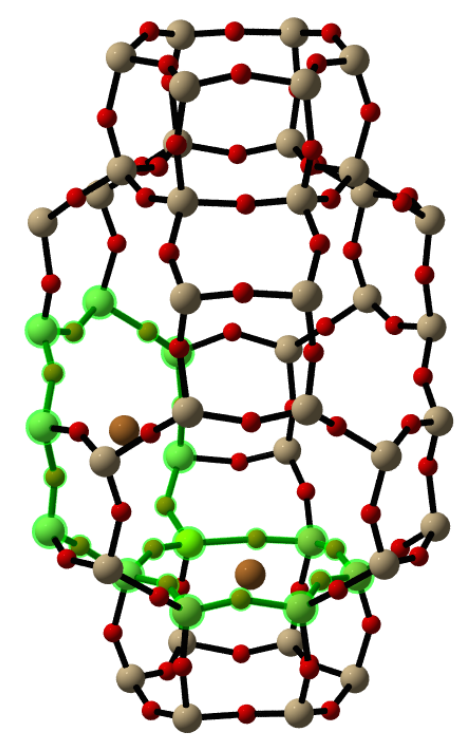

Figure 6.3.6 : Representation of the CHA topology and possible location of the cupper ions. 
The exact position of the cupper ions and their movement during the $\mathrm{NH}_{3}$-SCR reaction is an active research topic. ${ }^{451,452}$ First-principles simulations on extended clusters and periodic models of $\mathrm{Cu}$ SSZ-13 can nowadays both be performed using the DFT methodology. The exact location of the charge-compensating Al atoms in CU-SSZ-13 might influence its structure-function relationship, and whereas experimental investigations cannot provide further information on this, molecular modeling is ideally suited for this goal. Various authors have investigated the Al distribution in Cu-SSZ-13 and calculated structural and vibrational properties of the constructed models. Ribeiro and co-workers employed periodic DFT calculations using the PBE functional and reported that, in line with experiments, 4-fold $\mathrm{Cu}(\mathrm{II})$ and 2-fold $\mathrm{Cu}(\mathrm{l})$ emerge as the most stable $\mathrm{Cu}$ exchange sites. ${ }^{453} \mathrm{In}$ this study, the $\mathrm{Cu}$ ion is located near the top ring of the $\mathrm{d} 6 \mathrm{r}$ unit. A distribution of active sites was constructed by Goltl et al. using periodic simulations employing the hybrid HSEO6 functional . ${ }^{188}$ Eight different structures, including cupper in the 6- and 8-ring, were investigated and their corresponding energies vary over $135 \mathrm{~kJ} \mathrm{~mol}^{-1}$. Based on these energy considerations, the cupper is preferably located on top of the $d 6 r$ unit (see Figure 6.3.7). On the other hand, the preference for copper to reside in the 8-ring was reported by Anderson et al., pointing out that the Rietveld/maximum entropy method combined with DFT calculations suggest that almost $80 \%$ of the $\mathrm{Cu}$ ions are found in the 8-ring windows ${ }^{454}$.

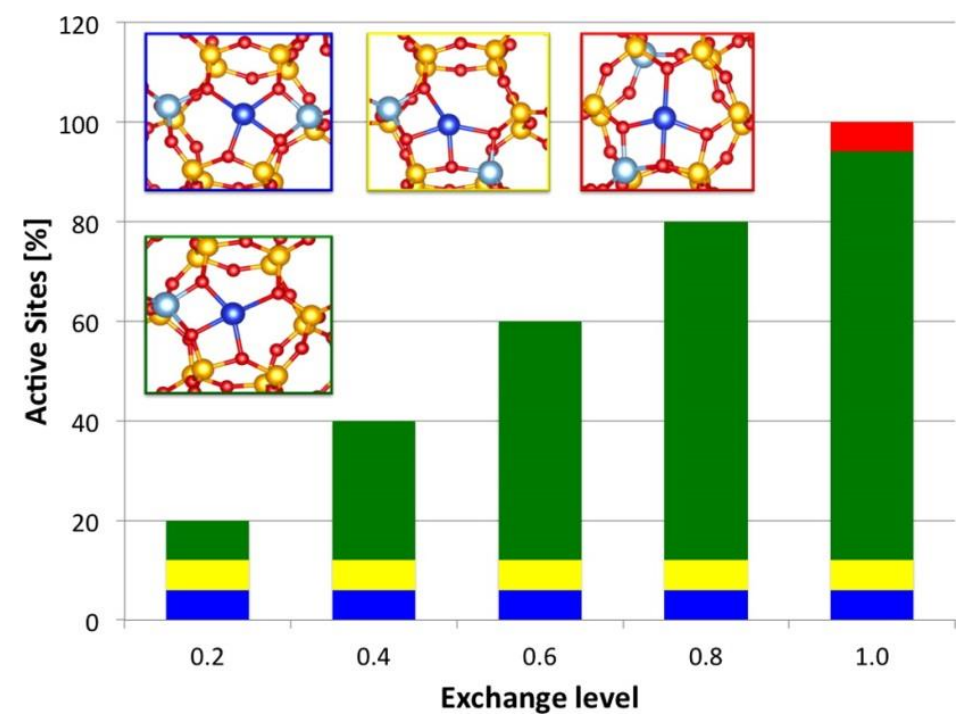

Figure 6.3.7 : Distribution of active sites in CU(II)-SSZ-13 at different exchange levels. Reprinted with permission from ref. ${ }^{188}$.

In addition to investigating the bare Cu-SSZ-13 materials, theoretical calculations are ideally suited to explore the geometries of the $\mathrm{Cu}$ coordinated complexes and their detailed molecular interactions. The coordination chemistry and reactivity of copper in zeolites was recently reviewed by Vanelderen 
et al., highlighting the crucial role of active dicopper cores in Cu-ZSM-5. ${ }^{455}$ Thus far, no dicopper species have been observed in the CHA topology and investigations focus on isolated copper sites . 449, 456 Adsorption of NH3 in Cu-SSZ-13 has been studied using various theoretical approaches. Giordanino and co-workers employed a $24 \mathrm{~T}$-site cluster representing the $d 6 r$ unit and reported an almost linear adsorption geometry in case of one ammonia molecule, and a N-Cu-N angle of $177^{\circ}$ in case of two ammonia molecules. ${ }^{457}$ The same authors extended their investigation to an $8 \mathrm{~T}$-site model representing the 8-ring. Computational screening of DFT-based optimized models via EXAFS fitting suggest the formation of tri-coordinated $[\mathrm{CuOH}]^{+}$complexes upon $\mathrm{O}_{2}$ activation, whereas bicoordinated $\mathrm{Cu}^{2+}$ cations present in the 8-ring windows are the dominant structures upon Heactivation. ${ }^{458}$ DFT-based Cu K-edge XANES calculations combined with EXAFS data suggested that the cupper ion, initially located on top of the $\mathrm{d} 6 \mathrm{r}$ unit, moves to a position slightly above this top plane upon $\mathrm{NH}_{3}$ adsorption. ${ }^{456}$ Similar conclusions were drawn by some of the presenting authors, using first-principles $\mathrm{MD}$ simulations and subsequent vibrational spectra of $\mathrm{NH}_{3}$-coordinated complexes to investigate the role of ammonia in the $\mathrm{NH}_{3}$-SCR reaction. ${ }^{459}$ In addition to Brønsted acid and Lewis acid sites, the presence of monovalent $\left[\mathrm{Cu}^{2+}(\mathrm{OH})^{-}\right]+$species, leading to a vibrational peak at circa $3660 \mathrm{~cm}^{-1}$, was demonstrated. Peaks in experimental FTIR spectra could be assigned based on the theoretical data, as shown in Table 6.3.1. The provided summary demonstrates that theoretical simulations on realistic model systems, with inclusion of the periodic nature of the catalyst material or accounting for typical process conditions, contribute substantially to the characterization of the active sites of Cu-SSZ-13 and the role of the SCR reactants such as $\mathrm{NH}_{3}$.

Table 6.3.1. Experimental and theoretical frequencies of $\mathrm{NH}_{3}$ species formed on the acid sites of CHA-type zeolites. Adapted with permission from ref. ${ }^{459}$.

\begin{tabular}{|l|l|l|l|}
\hline & Vexp $\left(\mathbf{c m}^{-1}\right)$ & Vtheo $\left(\mathbf{c m}^{-1}\right)$ & Assignment \\
\hline \multirow{2}{*}{$\mathrm{NH}_{3} @$ BAS (O2) } & $3400-3100$ & $3480-3200$ & $v\left(\mathrm{NH}_{4}{ }^{+}\right)$ \\
\cline { 2 - 4 } & $1455-1448$ & 1455 & $\delta\left(\mathrm{NH}_{4}{ }^{+}\right)_{\mathrm{as}}$ \\
\cline { 2 - 4 } & $1401-1393$ & 1368 & $\delta\left(\mathrm{NH}_{4}{ }^{+}\right)_{\mathrm{s}}$ \\
\hline$\left[\mathrm{Cu}\left(\mathrm{NH}_{3}\right)_{4}\right]^{2+}$ & $3400-3100$ & $3480-3200$ & $v\left(\mathrm{NH}_{3}\right)$ \\
\cline { 2 - 4 } & 1619 & 1606 & $\delta\left(\mathrm{NH}_{3}\right)$ \\
\cline { 2 - 4 } & 1278 & 1285 & $\mathrm{NH}_{3}$ wagging \\
\hline
\end{tabular}




\subsection{Molecular Dynamics for Diffusion of Sorbed Molecules}

Molecular sorption and diffusion are key fundamental processes in both catalysis and separation technologies. Diffusion is a phenomenon that takes place at larger length and time scales and is typically studied using force field based approaches. The derivation of the diffusion constant is well established using MD simulations, which track the position of the molecule over time. ${ }^{460} \mathrm{MD}$ employing force fields has made a major contribution to our understanding of these processes and has indeed been used extensively and effectively for modelling the sorption and diffusion of molecules within microporous materials; moreover, where the interactions between the sorbed molecule and the framework are non-bonded, "classical" MD is the more efficient and appropriate technique. A rich variety of information - both quantitative and qualitative - can be obtained from such simulations. Diffusion coefficients may be calculated and the simulations yield valuable information on migration mechanisms, resulting in the frequent use of MD simulations directly with experiment in investigating the dynamics of sorbed molecules in zeolites. Furthermore a proper understanding of diffusion is for some processes key towards understanding the product selectivity in catalytic processes, more information hereon will be given later in this section.

In this section, we outline the current status of MD methods and the role of the techniques in zeolite science, focusing on examples from the recent literature. Our discussion will concentrate of hydrocarbons sorbed in zeolites, which have received the greatest attention; but the techniques are generally applicable in this field.

\subsubsection{Techniques}

MD simulations of molecular processes in zeolites use standard techniques (see for example reference ${ }^{461}$ with forces calculated using the specified interatomic potential (or force field). For a comprehensive review on molecular simulations of diffusion and shape selectivity is available from Smit and Maesen. ${ }^{266}$ The main technical issues and choices relate to the following:

(i) The model used in describing the molecule- framework interactions.

(ii) The approach to framework dynamics.

Regarding the first area, the most significant choice is the level of granularity used in describing the sorbed molecule. Much earlier work used "united atom" models in which functional groups, for example, methyl and methylene groups are represented by a single particle - an approach that obviously reduces the computational requirements considerably compared with the alternative full atom approach in which all atoms are represented explicitly as a force field centre. The united atom approach has been employed extensively, especially in modelling the dynamics of saturated 
hydrocarbons, although recent work has found that it gives rise to significant errors in calculating diffusion coefficients for hydrocarbon molecules in zeolites, as will be discussed below.

With regard to framework dynamics, the choice is whether or not it should be included. Again, much earlier work used the "rigid framework" approximation in order to reduce computational cost. The approach is reasonable as the molecules and not the framework are diffusing. However, it has become increasingly clear that zeolite frameworks are far from rigid and that inclusion of framework flexibility is necessary for quantitative accuracy; and in some case its exclusion can result in significant qualitative effects. Potential models are required for the interactions between the framework atoms to include framework flexibility. Here there are two approaches: the first views the zeolite framework as a covalent network and sets up a "molecular mechanics" force field; the second is based on the Born model with electrostatic interactions between ions and non-bonded interactions. Since the nature of the bonding in zeolites (and other silicate systems) unquestionably has both ionic and covalent character, both approached are valid, although more recent work has tended to use the latter approach. More information on the usage of interatomic potentials can be used in a recent review of Sastre and co-workers. ${ }^{147}$

Having selected an approach, the next step is to implement the simulation in an MD code. Several general purpose codes have been used; the recent work of the present authors has generally employed the DL_POLY code which has the advantage of performing very efficiently on massively parallel (MPP) high performance computing (HPC) platforms. ${ }^{462}$ A crucial factor in any MD simulation and one that is critical in simulating diffusion in zeolites is the length of "real time" that can be sampled in the simulation. Diffusion coefficients are calculated using the Einstein relationship, by plotting the mean square atomic displacement (MSD) against time; a relevant example is shown in figure 6.4.1. It is essential that sufficient migration occurs during the time period sampled for a smooth plot with good statistics to be obtained, as indeed is the case in the example shown. With contemporary computing resources, simulations sampling several nanoseconds are routine and with HPC hundreds of ns are accessible; moreover, these time horizons continue to grow. However, even with simulations of this length, "slow" processes with high activation energies will not be accessible and alternative procedures, based, for example, on Kinetic Monte Carlo (KMC) are needed. In zeolite simulations long "real times" are particularly important to allow the diffusing molecules to explore fully the channel structures of the materials. If diffusion barriers are too high, diffusion itself becomes a rare event and a range of techniques have been suggested to treat diffusion also in these conditions, which we will discuss later in this section. ${ }^{463}$ 


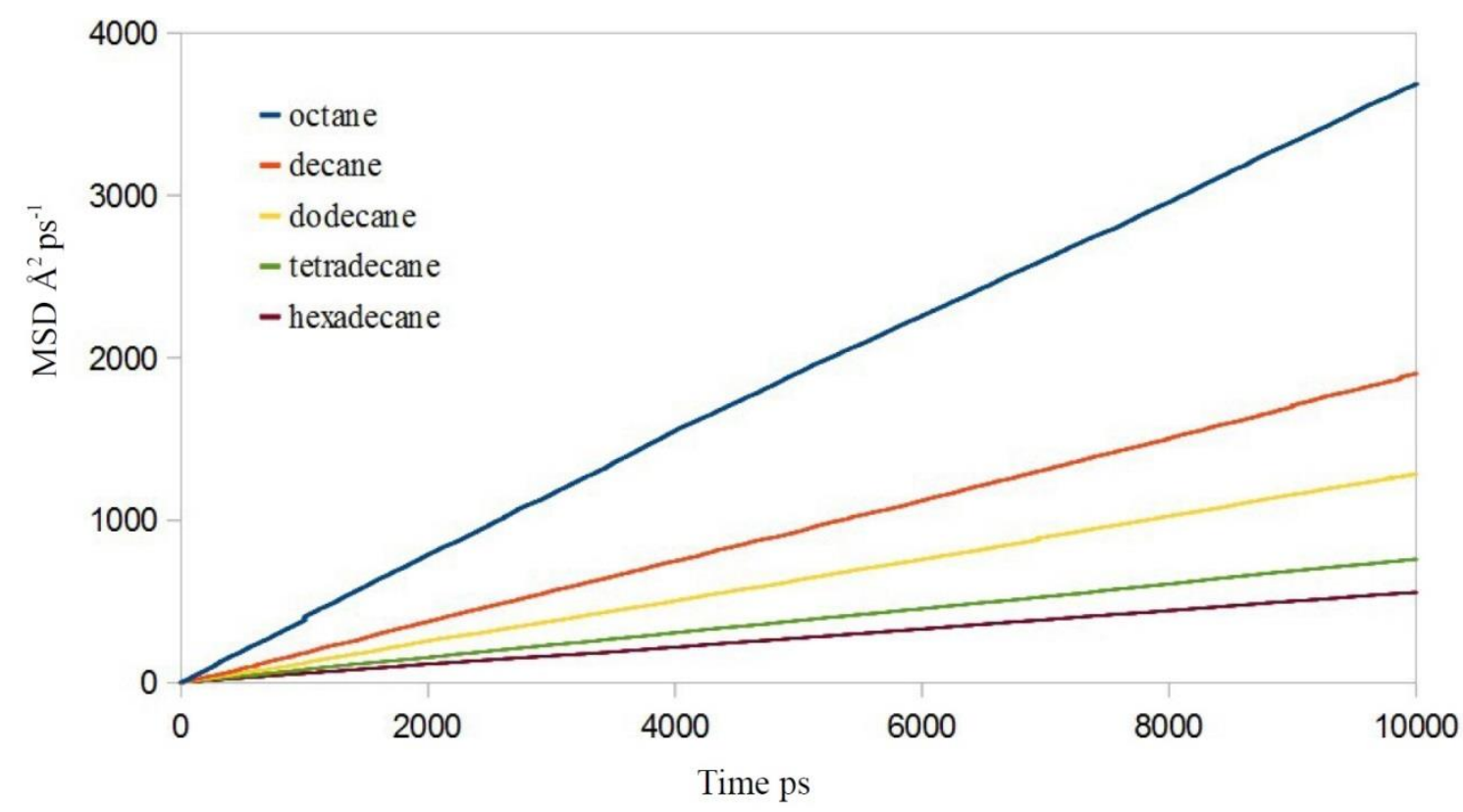

Figure 6.4.1: The average mean squared displacement as a function of time for all alkanes at 300 $\mathrm{K}$ in silicalite. Reprinted with permission from ref. ${ }^{464}$.

Interaction and comparison with experiment is clearly of key importance and the field has benefited from the detailed information available on diffusion processes from Quasi-Elastic Neutron Scattering (QENS), which can yield accurate diffusion coefficients that can be compared directly with simulation data and where the work of Jobic and co-workers ${ }^{465,466}$ on diffusion in zeolites has been particularly prominent

\subsubsection{Case Study: Linear Alkane Diffusion in Silicalite}

Alkane diffusion in zeolites has been widely studied owing both to its fundamental importance and to its relevance to hydrocarbon synthesis and cracking catalysis. Several MD studies reported useful information on both diffusion coefficients and mechanisms. ${ }^{466-468}$ However, in some cases, there were discrepancies of several orders of magnitude between calculated and experimental diffusion coefficients, with the simulations exaggerating the mobility of the sorbed hydrocarbons. The problem was re-examined in reference ${ }^{469}$ which critically evaluated the effects of the various approximations discussed above and found that to achieve improved agreement between calculated and experimental diffusion coefficients, it is necessary to include both framework flexibility and a full atom-atom description of the interaction between the hydrocarbon and the framework. The dynamical nature of the framework appreciably modifies that of the diffusing molecule, while the united atom model makes the interactions between the framework and the molecules too "smooth" thereby enhancing mobility. Figure 6.4 .2 shows the comparison between calculated diffusion coefficients for the different approximations with experimental QENS data from which it is clear that 
the united atom/ rigid framework approaches are major approximations which very significantly affect the resulting diffusion coefficients. ${ }^{466}$ The calculations also show the significant effects of loading, with higher diffusion rates being calculated for dilute systems, compared with those obtained for the loadings used in the experimental QENS studies. Two further features deserve comment. The first is that earlier "hierarchical" simulations perform well; these are based on a highly coarse grained approach, which clearly is effective, although the full atom-atom model which is now computationally feasible is more generally applicable. Secondly, we note that the simulated diffusion coefficients although much closer to experiment than the earlier studies, are still significantly higher than measured, which we attribute to the use of a "perfect" zeolite, whereas the real system will contain defects, which may significantly hinder molecular diffusion.

The use of the full atom model affects the simulations in a qualitative as well as a quantitative manner as is clear from Figure 6.4.3, which shows the variation in the calculated diffusion coefficient with hydrocarbon chain length for a variety of models. We see that the simpler models show an oscillatory variation, the origin of which has been discussed in earlier work, but which is no longer found in the full atom-atom model which shows a smooth monotonic variation.

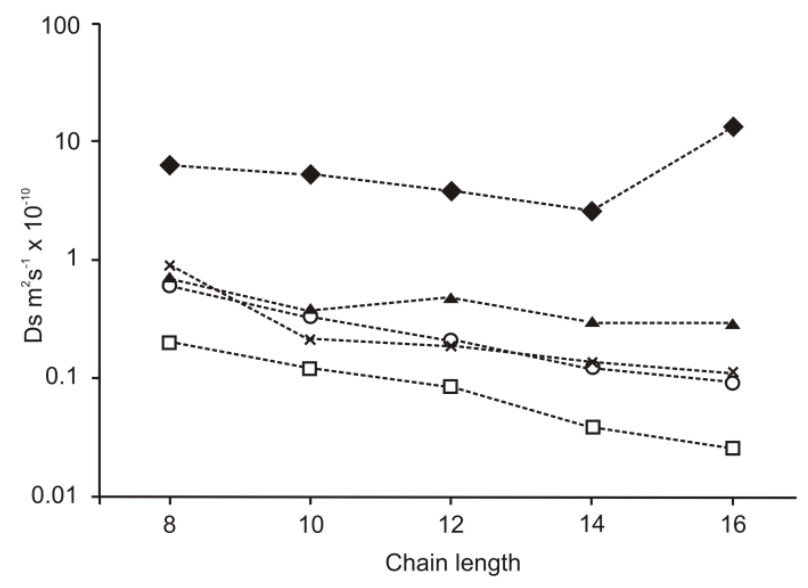

Figure 6.4.2: The calculated diffusion coefficients from the current MD simulations (O), filled triangles are results from MD simulations at infinite dilution, filled diamonds correspond to MD simulations using simpler models, results indicated with star are from Hierarchical simulations and squares are from QENS studies. 


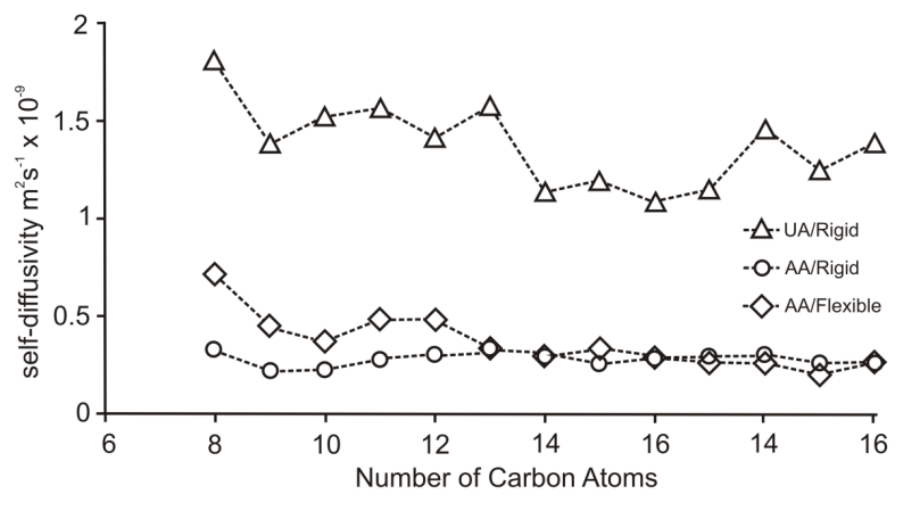

Figure 6.4.3 : Comparison of the united-atom/rigid framework simulation, all-atom/rigid framework simulation and all-atom/flexible framework simulation in terms of the dependence of diffusivity on chain length in the straight channels of silicalite. Reprinted with permission from ref. ${ }^{469}$.

The simulations are also of value in giving mechanistic insight. For example, in ref. ${ }^{469}$, simulations of octane in silicalite showed the intriguing phenomenon of channel switching between straight and sinusoidal channels at higher temperatures as illustrated in Figure 6.4.4. Indeed the simulations allow the diffusing molecules to explore the tortuosity of the channel structure.

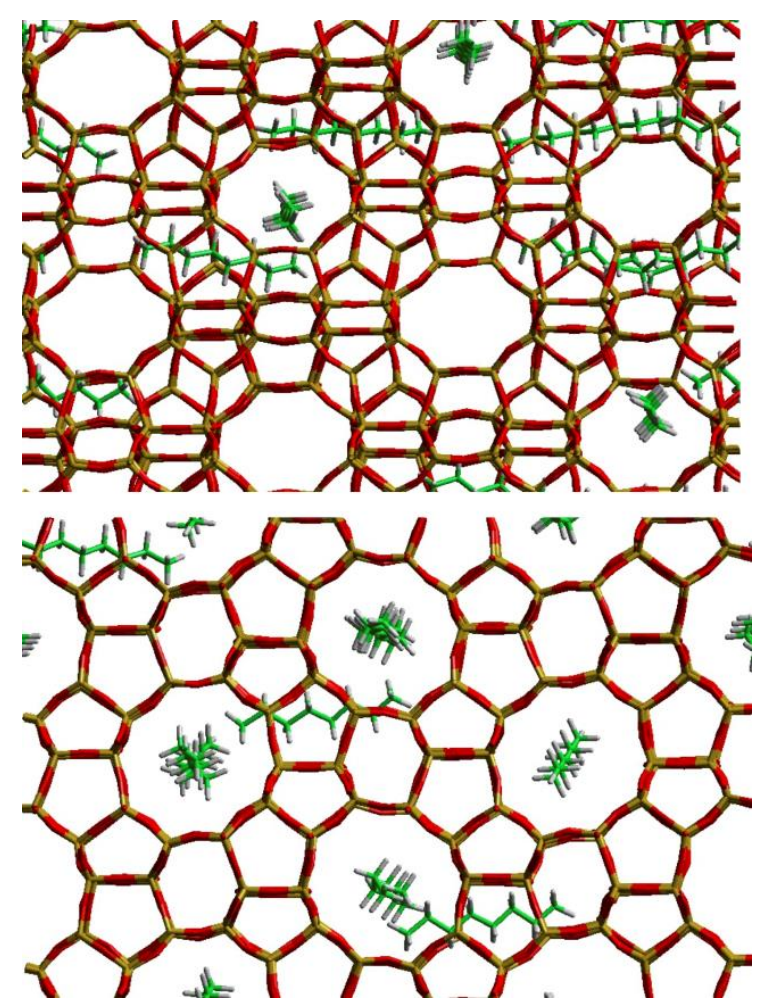

Figure 6.4.4: A snapshot of the MD simulation of $n$-octane at $400 \mathrm{~K}$ after $4 \mathrm{~ns}$, showing that 3 octane molecules have switched from the [010] channel system to the [001] channel system. Reprinted with permission from ref. ${ }^{469}$. 
Overall, the simulations show that with contemporary computer power we can now achieve increasingly close agreement between simulated and experimental diffusion coefficients and that the simulations are of great value in exploring both quantitative and qualitative aspects of the dynamics of sorbed molecules in zeolites.

\subsubsection{Diffusion in confinement}

For processes where diffusion barriers are too high, MD runs are too slow to efficiently generate particle trajectories over the time-scales of the diffusion process. ${ }^{470}$ As such diffusion itself becomes a rare event and may be viewed as a hopping process between large cages separated by narrow windows. A range of techniques have been suggested to also treat diffusion in these conditions, including dynamically corrected Transition State Theory, a kinetic Monte Carlo approach... ${ }^{463}$ The interested reader is further referred to the recent work of Smit et al. ${ }^{463}$ where self- and collective diffusion of adsorbed gases were studied in confined zeolite materials. When the kinetic diameter of the molecules becomes of the same order as the pore size of the zeolite, special care needs to be taken to treat the flexibility of the material appropriately. ${ }^{105}$ This is for example the case for small pore 8-ring zeolites with pore sizes of the order of 3-4 A which are important for separation of small molecules but also for catalysis using such small ring zeolites. Indeed diffusion has received an increased interest for example in the context of the Methanol to Olefin process, which was discussed in section 6.2.4. One of the archetypal catalysts is the small ring zeolite, H-SAPO-34, with the chabazite topology. Within the context of the MTO process H-SAPO-34 is such a small ring zeolite. The product distribution is critically dependent on the diffusion of products through the channels of the zeolite pore system.

Recently a series of studies appeared on the effects of framework flexibility on molecular diffusion in 8-ring zeolites and new efficient methods have been proposed to account for the flexibility in a computationally affordable way. ${ }^{471,472}$

Following the renewed interest of diffusion in the MTO chemistry, some MD studies appeared in which the diffusion of small molecules such as propane and propene were studied in various zeolites relevant for MTO purposes such as $\mathrm{CHA}, \mathrm{MFI}, \mathrm{BEA}$ and FAU ${ }^{473}$ and small pore zeolites with AFX and AEI topology. ${ }^{474}$ In the study of Sastre isostructural materials were investigated both having an aluminosilicate and an silicoaluminophoshate (SAPO) composition. Furthermore also Brønsted acidic sites were introduced in the framework. The study thus encompasses both the effect of composition and effect of distinct acidic sites on the diffusion of propane. The study shows that the diffusion of propane like molecules is indeed an activated process and distinct differences were found in terms of the acidity and proton positions. Figure 6.4 .5 shows trajectory plots of propane in 
both SSZ-16 and SAPO-56, two isostructural materials with the AFX topology. For the SSZ-16 only intra-cavity motions are observed at $600 \mathrm{~K}$ whereas for SAPO-56 some inter-cavity motions are detected at this temperature. These plots clearly show that the diffusion itself becomes a rare event. In these cases and a fortiori in cases where also blocked hydrocarbon pool species are present in the pores of the material, enhanced sampling methods would be very promising.
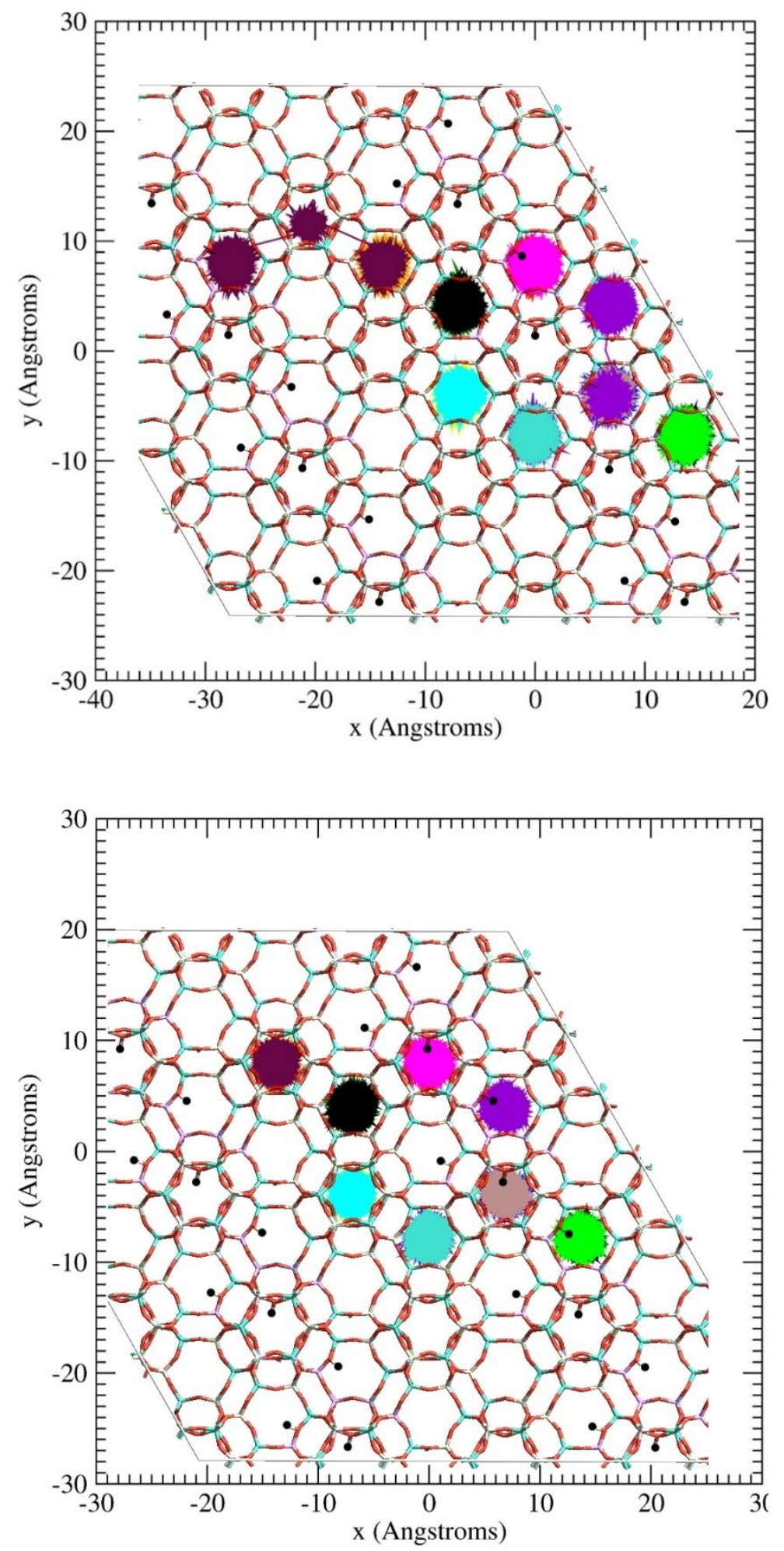
Figure 6.4.5 : Trajectories of propane molecules in SAPO-56 (top) and SSZ-16 (bottom). Intercavity jumps are only observed in the case of SAPO-56. Intercavity motions never happen through 8-rings containing protons (black balls). Reprinted with permission from ref. ${ }^{474}$.

The two case studies, given in this section, have briefly shown the possibilities and future challenges with regard to molecular simulations of diffusion of molecules within zeolites.

\section{Final discussion, summary and perspectives and outlook}

The examples in this review illustrate the contention in the Introduction that modelling is now contributing to many and indeed most of the fundamental and applied areas of zeolite science. The field has progressed enormously in the last three decades, enabled and driven by advances in both computational techniques and hardware. Some very general trends can be seen in the recent development of the zeolite modelling, of which the following are perhaps the most significant:

- Growth in accuracy, where computational methods can now achieve accurate quantitative agreement with experiment measurements of several observables, which in turn leads to a greater predictive capacity for the field.

- Increased complexity of the systems that are amenable to modelling methods, which in turn leads to greater detail and realism in the resulting models.

- Growing integration of the different modelling approaches, enabling the modelling of whole processes such as reaction kinetics and crystal growth.

- Increasingly close interaction with experiment resulting in modelling methods being used as tools together with structural and spectroscopic methods - a feature now of several areas of both physical and biological sciences.

Some applications of modelling methods are now indeed routine, although others remain challenging. Crystal structures can be modelled straightforwardly and accurately with both interatomic potentials and electronic structure based methods; structure prediction, where great progress has been made, still, however, remains far from routine. The structures and energetics of silicate clusters can again be calculated to a good level of accuracy, but the treatment of solvation, despite the extensive developments of recent years is difficult, as is the stage beyond of integrating such calculations into kinetic models of crystal growth.

Modelling of spectroscopic properties is experiencing methodological breakthroughs which are more and more available for realistic zeolite models. In particular, time-dependent density functional 
theory comes within reach and can be used for excited state phenomena. Vibrational information can be derived via MD simulations and hence provides insight on the growth mechanism or structural characteristics of zeolite materials.

Modelling of reaction mechanisms in catalysis has made enormous strides and as shown in several case studies, modelling tools have proven their indispensable role to elucidate complex reaction schemes. Indeed detailed understanding of zeolite catalysis at the molecular scale is prerequisite to optimize current large scale industrial processes but also vital in assisting the development of emerging applications. Active research is currently performed to extend the applicability range of zeolites towards conversion of biomass and biomass derived feedstocks to valuable products. 4,475 Such ambitious goals can only be obtained from complementary insights from theory, experiment, spectroscopy.... Substantial progress has been made in developing kinetic models, which, however, place great demands on the accuracy of the calculated activation energies and more general kinetic data for elementary steps. A major leap has been made in this field the last decade. Theoretical techniques have matured to a level that near chemical accuracy can be obtained for the chemical kinetics of elementary reactions provided the active site and mechanism is known. Future challenges and methodological improvements will be necessary to deal with the complexity of a chemical transformation as encountered at experimental conditions.

Sorption isotherms and sorbate diffusion coefficients can be calculated with increased accuracy, but in some cases there is a need for more realistic models of the zeolite hosts, relating to defects and extra-framework species. Furthermore an ingenious coupling between modelling results at various length and time scales will be necessary. For example diffusion has been acknowledged as a major factor in the determination of the observed selectivity and the overall molecular understanding of selectivity should ideally couple insights from the reaction kinetics and diffusional processes.

There remain, of course, many challenges, of which the following are clear both from the comments above and from the examples in our review:

- Modelling of solvation - a widespread problem in computational chemistry and biomolecular modelling - is, as we have commented, difficult. Substantial progress has been made and growth in computer power is leading to increased use of quantum mechanically described explicit solvent models, but there remain many problems, which will need to be solved if we are to obtain a detailed understanding of the chemistry of synthesis gels. Molecular dynamics simulations accounting for the full molecular environment are increasingly popular. However given the diversity of species in the reaction medium and the wide range of characteristic time scale encountered in the overall process, major methodological and computational challenges remain to be solved. 
- Calculation of accurate activation energies and kinetic data of elementary reaction steps. As our review has shown, there has been considerable progress, providing currently matured methods that allow highly accurate predictions. Continuous efforts should be undertaken to eventually assess the error bars on first principle derived kinetic data that are to be used in kinetic modelling schemes to permit reliable quantitative predictions.

- Theoretical simulation of complex chemical transformations accounting for experimental conditions. At operating conditions chemical reactions taking place at the nanometer scale have a very complex nature due to the interplay between various correlated factors, such as framework flexibility, competitive pathways, various guest particles present in the pores of the material. Proper theoretical treatment of reactions under these conditions will require smart solutions from theoreticians.

- Closer integration of different modelling methods. We hope that progress in this important area has emerged as one of the themes of this review, but there is a need for extensive further developments.

- Calculation of accurate excitation and emission spectra. Further developments in the field of excited-state calculations are needed to account for the influence of the porous zeolitic environment on the spectroscopic properties of reaction intermediates or dyes exhibiting optical activity. Advances in TD-DFT, as well as other theoretical methodologies are necessary to describe these complex host-guest complexes.

- Coarser grained modelling methods will need to be developed and integrated with molecular modelling in, for example, the modelling of crystal growth.

- Use of "real" models for the zeolite host. Zeolites are far from perfect periodic systems. They contain defects - both point and extended - as well, often, as ill-defined extraframework species. These species may have a major influence on the properties and reactivities of the materials.

Similar problems are encountered in many other areas of computational science, especially biomolecular modelling and indeed our field will benefit from progress made here and in computational chemistry and materials science more generally. The problems we have outlined are soluble and the field has indeed an exciting future in which developments in methodology and continuing expansion of computer power will move applications increasingly from system to process modelling and in which modelling methods will become ever more closely integrated with experiment.

\section{Acknowledgements}


V. Van Speybroeck, K. Hemelsoet, L. Joos and M. Waroquier acknowledge all current and former $\mathrm{CMM}$ members for contributions to the work reviewed and for general discussions and the Fund for Scientific Research - Flanders (FWO), the Research Board of Ghent University (BOF) and BELSPO in the frame of IAP/7/05 for financial support. V. Van Speybroeck acknowledges funding from the European Research Council under the European Community's Seventh Framework Programme (FP7(2007-2013) ERC grant agreement number 240483), and from the European Union's Horizon 2020 research and innovation programme (consolidator ERC grant agreement No 647755 - DYNPOR (2015-2020)).

All authors acknowledge Wim Dewitte for technical support with the figures.

R.G. Bell acknowledges Michael Fischer and Martijn Zwijnenburg and C.R.A. Catlow acknowledges Furio Cora, Ben Slater, Dewi Lewis, Dave Willock, Graham Hutchings, Miguel Mora-Fonz, Alexei Sokol, Luis Gomex-Hortiguela, Gopinathan Sankar, John Meurig Thomas and Alex O'Malley for contributions to the work reviewed and for general discussion on zeolite science. R.G. Bell and C.R.A. Catlow acknowledge support by several grants from EPSRC and the EU and are grateful for collaboration and support from Johnson Matthey.

\section{References}

1. S. M. Woodley and C. R. A. Catlow, Physical Chemistry Chemical Physics, 2014, 16, 2100121001.

2. W. A. de Jong, E. Bylaska, N. Govind, C. L. Janssen, K. Kowalski, T. Mueller, I. M. B. Nielsen, H. J. J. van Dam, V. Veryazov and R. Lindh, Physical Chemistry Chemical Physics, 2010, 12, 68966920.

3. R. Chal, C. Gerardin, M. Bulut and S. van Donk, Chemcatchem, 2011, 3, 67-81.

4. P. A. Jacobs, M. Dusselier and B. F. Sels, Angewandte Chemie-International Edition, 2014, 53, 8621-8626.

5. J. Gascon, F. Kapteijn, B. Zornoza, V. Sebastian, C. Casado and J. Coronas, Chemistry of Materials, 2012, 24, 2829-2844.

6. W.-g. Kim and S. Nair, Chemical Engineering Science, 2013, 104, 908-924.

7. M. Pera-Titus, Chemical Reviews, 2014, 114, 1413-1492.

8. S. M. Auerbach, K. A. Carrado and P. K. Dutta, CRC Press, 2003.

9. J. Weitkamp and L. Puppe, Catalysis and Zeolites, Springer 1999.

10. B. M. Weckhuysen, Angewandte Chemie-International Edition, 2009, 48, 4910-4943.

11. I. L. C. Buurmans and B. M. Weckhuysen, Nature Chemistry, 2012, 4, 873-886.

12. S. Raimondeau and D. G. Vlachos, Chem. Eng. J., 2002, 90, 3-23.

13. N. Lopez, N. Almora-Barrios, G. Carchini, P. Blonski, L. Bellarosa, R. Garcia-Muelas, G. NovellLeruth and M. Garcia-Mota, Catalysis Science \& Technology, 2012, 2, 2405-2417.

14. F. J. Keil, in Multiscale Molecular Methods in Applied Chemistry, eds. B. Kirchner and J. Vrabec, 2012, vol. 307, pp. 69-107.

15. C. E. Gounaris, C. A. Floudas and J. Wei, Chemical Engineering Science, 2006, 61, 7933-7948.

16. C. E. Gounaris, J. Wei and C. A. Floudas, Chemical Engineering Science, 2006, 61, 7949-7962.

17. C. E. Gounaris, J. Wei, C. A. Floudas, R. Ranjan and M. Tsapatsis, Aiche J., 2010, 56, 611-632.

18. E. L. First, C. E. Gounaris, J. Wei and C. A. Floudas, Physical Chemistry Chemical Physics, 2011, 13, 17339-17358. 
19. Structure Commission of the International Zeolite Association (IZA-SC), Database of Zeolite structures http://www.iza-structure.org/databases/.

20. B. Smit and T. L. M. Maesen, Nature, 2008, 451, 671-678.

21. S. T. Wilson, B. M. Lok, C. A. Messina, T. R. Cannan and E. M. Flanigen, Journal of the American Chemical Society, 1982, 104, 1146-1147.

22. T. Yanagisawa, T. Shimizu, K. Kuroda and C. Kato, Bulletin of the Chemical Society of Japan, 1990, 63, 988-992.

23. A. Corma, Chemical Reviews, 1997, 97, 2373-2419.

24. C. Martinez and A. Corma, Coordination Chemistry Reviews, 2011, 255, 1558-1580.

25. D. E. De Vos, M. Dams, B. F. Sels and P. A. Jacobs, Chemical Reviews, 2002, 102, 3615-3640.

26. A. Primo and H. Garcia, Chemical Society Reviews, 2014, 43, 7548-7561.

27. W. Vermeiren and J. P. Gilson, Topics in Catalysis, 2009, 52, 1131-1161.

28. T. F. Degnan, Journal of Catalysis, 2003, 216, 32-46.

29. C. E. A. Kirschhock, R. Ravishankar, F. Verspeurt, P. J. Grobet, P. A. Jacobs and J. A. Martens, Journal of Physical Chemistry B, 2002, 106, 3333-3334.

30. C. T. G. Knight and S. D. Kinrade, Journal of Physical Chemistry B, 2002, 106, 3329-3332.

31. J. C. G. Pereira, C. R. a. Catlow and G. D. Price, Chemical Communications, 1998, 1387-1388.

32. J. C. G. Pereira, C. R. a. Catlow and G. D. Price, The Journal of Physical Chemistry A, 1999, 103, 3252-3267.

33. J. C. G. Pereira, C. R. a. Catlow and G. D. Price, The Journal of Physical Chemistry A, 1999, 103, 3268-3284.

34. J. C. G. Pereira, C. R. A. Catlow and G. D. Price, Journal of Physical Chemistry A, 2002, 106, 130-148.

35. A. Klamt, Journal of Physical Chemistry, 1995, 99, 2224-2235.

36. J. Tomasi, B. Mennucci and R. Cammi, Chemical Reviews, 2005, 105, 2999-3093.

37. M. J. Mora-Fonz, C. R. a. Catlow and D. W. Lewis, Angewandte Chemie (International ed. in English), 2005, 44, 3082-3086.

38. M. J. Mora-Fonz, C. R. a. Catlow and D. W. Lewis, Journal of Physical Chemistry C, 2007, 111, $18155-18158$.

39. M. J. Mora-Fonz, C. R. a. Catlow and D. W. Lewis, Physical chemistry chemical physics : PCCP, 2008, 10, 6571-6578.

40. M. J. Mora-Fonz, S. Hamad and C. R. A. Catlow, Molecular Physics, 2007, 105, 177-187.

41. C. R. A. Catlow, S. T. Bromley, S. Hamad, M. Mora-Fonz, A. A. Sokol and S. M. Woodley, Physical Chemistry Chemical Physics, 2010, 12, 786-811.

42. G. J. McIntosh, Physical Chemistry Chemical Physics, 2012, 14, 996-1013.

43. G. J. McIntosh, Physical Chemistry Chemical Physics, 2013, 15, 17496-17509.

44. G. J. McIntosh, Physical Chemistry Chemical Physics, 2013, 15, 3155-3172.

45. H. Henschel, A. M. Schneider and M. H. Prosenc, Chemistry of Materials, 2010, 22, 51055111.

46. T. T. Trinh, A. P. J. Jansen, R. A. van Santen and E. J. Meijer, Physical Chemistry Chemical Physics, 2009, 11, 5092-5099.

47. T. T. Trinh, A. P. J. Jansen, R. A. van Santen, J. VandeVondele and E. J. Meijer, Chemphyschem, 2009, 10, 1775-1782.

48. A. Pavlova, T. T. Trinh, R. A. van Santen and E. J. Meijer, Physical Chemistry Chemical Physics, 2013, 15, 1123-1129.

49. T. T. Trinh, X. Rozanska, F. Delbecq and P. Sautet, Physical Chemistry Chemical Physics, 2012, 14, 3369-3380.

50. T. T. Trinh, A. P. J. Jansen, R. A. van Santen and E. J. Meijer, Journal of Physical Chemistry C, 2009, 113, 2647-2652.

51. C.-S. Yang, J. Miguel Mora-Fonz and C. R. A. Catlow, Journal of Physical Chemistry C, 2013, 117, 24796-24803. 
52. A. Alberti and A. Martucci, Journal of Physical Chemistry C, 2010, 114, 7767-7773.

53. A. Pavlova and E. J. Meijer, Chemphyschem, 2012, 13, 3492-3496.

54. L. Sutto, S. Marsili and F. Luigi Gervasio, Wiley Interdisciplinary Reviews-Computational Molecular Science, 2012, 2, 771-779.

55. T. S. Van Erp, T. P. Caremans, C. E. A. Kirschhock and J. A. Martens, Physical Chemistry Chemical Physics, 2007, 9, 1044-1051.

56. E. A. Carter, G. Ciccotti, J. T. Hynes and R. Kapral, Chemical Physics Letters, 1989, 156, 472477.

57. A. Malani, S. M. Auerbach and P. A. Monson, Journal of Physical Chemistry C, 2011, 115, 15988-16000.

58. X.-Q. Zhang, T. T. Trinh, R. A. van Santen and A. P. J. Jansen, Journal of Physical Chemistry C, 2011, 115, 9561-9567.

59. X.-Q. Zhang, T. T. Trinh, R. A. van Santen and A. P. J. Jansen, Journal of the American Chemical Society, 2011, 133, 6613-6625.

60. X.-Q. Zhang, R. A. van Santen and A. P. J. Jansen, Physical Chemistry Chemical Physics, 2012, 14, 11969-11973.

61. R. Brent, P. Cubillas, S. M. Stevens, K. E. Jelfs, A. Umemura, J. T. Gebbie, B. Slater, O. Terasaki, M. A. Holden and M. W. Anderson, Journal of the American Chemical Society, 2010, 132, 13858-13868.

62. Z. Liu, N. Fujita, K. Miyasaka, L. Han, S. M. Stevens, M. Suga, S. Asahina, B. Slater, C. Xiao, Y. Sakamoto, M. W. Anderson, R. Ryoo and O. Terasaki, Microscopy, 2013, 62, 109-146.

63. L. I. Meza, M. W. Anderson, B. Slater and J. R. Agger, Physical Chemistry Chemical Physics, 2008, 10, 5066-5076.

64. B. Slater, C. R. A. Catlow, Z. Liu, T. Ohsuna, O. Terasaki and M. A. Camblor, Angewandte Chemie-International Edition, 2002, 41, 1235-1237.

65. M. E. Chiu, B. Slater and J. D. Gale, Angewandte Chemie-International Edition, 2005, 44, 1213-1217.

66. B. Slater, J. D. Gale, C. R. A. Catlow, T. Ohsuna and O. Terasaki, in Recent Advances in the Science and Technology of Zeolites and Related Materials, Pts a - C, eds. E. VanSteen, M. Claeys and L. H. Callanan, 2004, vol. 154, pp. 1197-1203.

67. M. G. Wu and M. W. Deem, Journal of Chemical Physics, 2002, 116, 2125-2137.

68. S. X. M. Boerrigter, G. P. H. Josten, J. van de Streek, F. F. A. Hollander, J. Los, H. M. Cuppen, P. Bennema and H. Meekes, Journal of Physical Chemistry A, 2004, 108, 5894-5902.

69. P. Cubillas, S. M. Stevens, N. Blake, A. Umemura, C. B. Chong, O. Terasaki and M. W. Anderson, Journal of Physical Chemistry C, 2011, 115, 12567-12574.

70. P. Cubillas, J. T. Gebbie, S. M. Stevens, N. Blake, A. Umemura, O. Terasaki and M. W. Anderson, Journal of Physical Chemistry C, 2014, 118, 23092-23099.

71. D. W. Lewis, C. M. Freeman and C. R. A. Catlow, Journal of Physical Chemistry, 1995, 99, 11194-11202.

72. R. G. Bell, D. W. Lewis, P. Voigt, C. M. Freeman, J. M. Thomas and C. R. A. Catlow, in Zeolites and Related Microporous Materials: State of the Art 1994, eds. J. Weitkamp, H. G. Karge, H. Pfeifer and W. Holderich, 1994, vol. 84, pp. 2075-2082.

73. D. W. Lewis, D. J. Willock, C. R. A. Catlow, J. M. Thomas and G. J. Hutchings, Nature, 1996, 382, 604-606.

74. D. W. Lewis, G. Sankar, J. K. Wyles, J. M. Thomas, C. R. A. Catlow and D. J. Willock, Angewandte Chemie-International Edition, 1997, 36, 2675-2677.

75. G. Sankar, J. K. Wyles, R. H. Jones, J. M. Thomas, C. R. A. Catlow, D. W. Lewis, W. Clegg, S. J. Coles and S. J. Teat, Chemical Communications, 1998, 117-118.

76. L. Gomez-Hortigueela, F. Cora, G. Sankar, C. M. Zicovich-Wilson and C. R. A. Catlow, Chemistry-a European Journal, 2010, 16, 13638-13645. 
77. L. Gomez-Hortigueela, S. Hamad, F. Lopez-Arbeloa, A. B. Pinar, J. Perez-Pariente and F. Cora, Journal of the American Chemical Society, 2009, 131, 16509-16524.

78. L. Gomez-Hortiguela, R. Garcia, F. Lopez-Arbeloa, F. Cora and J. Perez-Pariente, Journal of Physical Chemistry C, 2010, 114, 8320-8327.

79. L. Gomez-Hortiguela, F. Cora, C. R. A. Catlow and J. Perez-Pariente, Physical Chemistry Chemical Physics, 2006, 8, 486-493.

80. L. Gomez-Hortigueela, C. Marquez-Alvarez, F. Cora, F. Lopez-Arbeloa and J. Perez-Pariente, Chemistry of Materials, 2008, 20, 987-995.

81. L. Gomez-Hortiguela, F. Cora and J. Perez-Pariente, Microporous and Mesoporous Materials, 2008, 109, 494-504.

82. T. Verstraelen, B. M. Szyja, D. Lesthaeghe, R. Declerck, V. Van Speybroeck, M. Waroquier, A. P. J. Jansen, A. Aerts, L. R. A. Follens, J. A. Martens, C. E. A. Kirschhock and R. A. van Santen, Topics in Catalysis, 2009, 52, 1261-1271.

83. C. E. A. Kirschhock, R. Ravishankar, L. Van Looveren, P. A. Jacobs and J. A. Martens, Journal of Physical Chemistry B, 1999, 103, 4972-4978.

84. C. E. A. Kirschhock, R. Ravishankar, F. Verspeurt, P. J. Grobet, P. A. Jacobs and J. A. Martens, Journal of Physical Chemistry B, 1999, 103, 4965-4971.

85. T. Verstraelen, V. Van Speybroeck and M. Waroquier, J. Chem Inf. Model., 2008, 48, 15301541.

86. M. J. Wulfers and F. C. Jentoft, Acs Catalysis, 2014, 4, 3521-3532.

87. S. Ilias and A. Bhan, Journal of Catalysis, 2014, 311, 6-16.

88. R. Pophale, P. A. Cheeseman and M. W. Deem, Physical Chemistry Chemical Physics, 2011, 13, 12407-12412.

89. M. M. J. Treacy, K. H. Randall, S. Rao, J. A. Perry and D. J. Chadi, Zeitschrift Fur Kristallographie, 1997, 212, 768-791.

90. M. W. Deem, R. Pophale, P. A. Cheeseman and D. J. Earl, Journal of Physical Chemistry C, 2009, 113, 21353-21360.

91. D. J. Earl and M. W. Deem, Industrial \& Engineering Chemistry Research, 2006, 45, 54495454.

92. Y. Li and J. H. Yu, Chemical Reviews, 2014, 114, 7268-7316.

93. O. D. Friedrichs, A. W. M. Dress, D. H. Huson, J. Klinowski and A. L. Mackay, Nature, 1999, 400, 644-647.

94. M. D. Foster, O. D. Friedrichs, R. G. Bell, F. A. A. Paz and J. Klinowski, Angewandte ChemieInternational Edition, 2003, 42, 3896-3899.

95. M. D. Foster, O. D. Friedrichs, R. G. Bell, F. A. A. Paz and J. Klinowski, Journal of the American Chemical Society, 2004, 126, 9769-9775.

96. M. D. Foster, A. Simperler, R. G. Bell, O. D. Friedrichs, F. A. A. Paz and J. Klinowski, Nature Materials, 2004, 3, 234-238.

97. D. Majda, F. A. A. Paz, O. D. Friedrichs, M. D. Foster, A. Simperler, R. G. Bell and J. Klinowski, Journal of Physical Chemistry C, 2008, 112, 1040-1047.

98. A. Simperler, M. D. Foster, O. D. Friedrichs, R. G. Bell, F. A. A. Paz and J. Klinowski, Acta Crystallographica Section B-Structural Science, 2005, 61, 263-279.

99. E. Garcia-Perez, D. Dubbeldam, B. Liu, B. Smit and S. Calero, Angewandte ChemieInternational Edition, 2007, 46, 276-278.

100. J. Kim, L. C. Lin, J. A. Swisher, M. Haranczyk and B. Smit, Journal of the American Chemical Society, 2012, 134, 18940-18943.

101. J. D. Gale, Journal of the Chemical Society-Faraday Transactions, 1997, 93, 629-637.

102. M. J. Sanders, M. Leslie and C. R. A. Catlow, Journal of the Chemical Society-Chemical Communications, 1984, 1271-1273.

103. M. A. Zwijnenburg, A. Simperler, S. A. Wells and R. G. Bell, Journal of Physical Chemistry B, $2005,109,14783-14785$. 
104. Y. Li, J. Yu and R. Xu, Angewandte Chemie-International Edition, 2013, 52, 1673-1677.

105. V. Kapko, C. Dawson, M. M. J. Treacy and M. F. Thorpe, Physical chemistry chemical physics : PCCP, 2010, 12, 8531-8541.

106. A. Sartbaeva, S. Wells, M. Treacy and M. Thorpe, Nature Materials, 2006, 5, 962-965.

107. C. Dawson, V. Kapko, M. Thorpe, M. Foster and M. Treacy, Journal of Physical Chemistry C, 2012, 116, 16175-16181.

108. V. A. Blatov, G. D. Ilyushin and D. M. Proserpio, Chemistry of Materials, 2013, 25, 412-424.

109. N. A. Anurova, V. A. Blatov, G. D. Ilyushin and D. M. Proserpio, Journal of Physical Chemistry C, 2010, 114, 10160-10170.

110. C. Baerlocher and L. B. McCusker, Database of Zeolite Structures, http://www.izastructure.org/databases/.

111. E. Verheyen, L. Joos, K. Van Havenbergh, E. Breynaert, N. Kasian, E. Gobechiya, K. Houthoofd, C. Martineau, M. Hinterstein, F. Taulelle, V. Van Speybroeck, M. Waroquier, S. Bals, G. Van Tendeloo, C. E. A. Kirschhock and J. A. Martens, Nature Materials, 2012, 11, 1059-1064.

112. E. Verheyen, L. Joos, C. Martineau, C. J. Dawson, C. Weidenthaler, W. Schmidt, R. Yuan, E. Breynaert, V. Van Speybroeck, M. Waroquier, F. Taulelle, M. Treacy, J. A. Martens and C. E. A. Kirschhock, Mater. Horiz. , 2014, 1, 582 - 587.

113. W. J. Roth and J. Cejka, Catalysis Science \& Technology, 2011, 1, 43-53.

114. W. J. Roth, P. Nachtigall, R. E. Morris, P. S. Wheatley, V. R. Seymour, S. E. Ashbrook, P. Chlubna, L. Grajciar, M. Polozij, A. Zukal, O. Shvets and J. Cejka, Nature Chemistry, 2013, 5, 628-633.

115. M. Trachta, O. Bludsky, J. Cejka, R. E. Morris and P. Nachtigall, Chemphyschem, 2014, 15, 2972-2976.

116. T. Blasco, A. Corma, M. J. Diaz-Cabanas, F. Rey, J. Rius, G. Sastre and J. A. Vidal-Moya, Journal of the American Chemical Society, 2004, 126, 13414-13423.

117. G. Sastre, J. A. Vidal-Moya, T. Blasco, J. Rius, J. L. Jorda, M. T. Navarro, F. Rey and A. Corma, Angewandte Chemie-International Edition, 2002, 41, 4722-4726.

118. G. Sastre, A. Pulido and A. Corma, Microporous and Mesoporous Materials, 2005, 82, 159163.

119. P. Kamakoti and T. A. Barckholtz, Journal of Physical Chemistry C, 2007, 111, 3575-3583.

120. G. Sastre and J. D. Gale, Chemistry of Materials, 2003, 15, 1788-1796.

121. G. Sastre and J. D. Gale, in Recent Advances in the Science and Technology of Zeolites and Related Materials, Pts a - C, eds. E. VanSteen, M. Claeys and L. H. Callanan, 2004, vol. 154, pp. 1261-1266.

122. L. Grajciar, O. Bludsky, W. J. Roth and P. Nachtigall, Catalysis Today, 2013, 204, 15-21.

123. J. Hermann, M. Trachta, P. Nachtigall and O. Bludsky, Catalysis Today, 2014, 227, 2-8.

124. W. J. Roth, P. Nachtigall, R. E. Morris and J. Cejka, Chemical Reviews, 2014, 114, 4807-4837.

125. G. Piccini and J. Sauer, Journal of Chemical Theory and Computation, 2013, 9, 5038-5045.

126. C. J. Dawson, V. Kapko, M. F. Thorpe, M. D. Foster and M. M. J. Treacy, The Journal of Physical Chemistry C, 2012, 116, 16175-16181.

127. E. Verheyen, S. P. Sree, K. Thomas, J. Dendooven, M. De Prins, G. Vanbutsele, E. Breynaert, J. P. Gilson, C. E. A. Kirschhock, C. Detavernier and J. A. Martens, Chemical Communications, 2014, 50, 4610-4612.

128. L. Whitmore, B. Slater and C. R. A. Catlow, Physical Chemistry Chemical Physics, 2000, 2, 5354-5356.

129. C. R. A. Catlow, R. G. Bell, J. D. Gale and D. W. Lewis, in Zeolites: A Refined Tool for Designing Catalytic Sites, eds. L. Bonneviot and S. Kaliaguine, 1995, vol. 97, pp. 87-100.

130. N. E. R. Zimmermann, S. Jakobtorweihen, E. Beerdsen, B. Smit and F. J. Keil, The Journal of Physical Chemistry C, 2007, 111, 17370-17381.

131. J. Sauer and H.-J. Freund, Catalysis Letters, 2015, 145, 10.1007/s10562-10014-11387-10561. 
132. B. M. Weckhuysen, Chemical Society Reviews, 2010, 39, 4557-4559.

133. B. A. De Moor, A. Ghysels, M.-F. Reyniers, V. Van Speybroeck, M. Waroquier and G. B. Marin, Journal of Chemical Theory and Computation, 2011, 7, 1090-1101.

134. A. Ghysels, V. Van Speybroeck, E. Pauwels, S. Catak, B. R. Brooks, D. Van Neck and M. Waroquier, Journal of Computational Chemistry, 2010, 31, 994-1007.

135. G. Piccini and J. Sauer, Journal of Chemical Theory and Computation, 2014, 10, 2479-2487.

136. P. Bornhauser and D. Bougeard, Journal of Physical Chemistry B, 2001, 105, 36-41.

137. K. S. Smirnov and D. Bougeard, Journal of Physical Chemistry, 1993, 97, 9434-9440.

138. A. J. M. Deman and R. A. van Santen, Zeolites, 1992, 12, 269-279.

139. K. S. Smirnov and D. Bougeard, Catalysis Today, 2001, 70, 243-253.

140. D. Lesthaeghe, P. Vansteenkiste, T. Verstraelen, A. Ghysels, C. E. A. Kirschhock, J. A. Martens, V. Van Speybroeck and M. Waroquier, Journal of Physical Chemistry C, 2008, 112, 91869191.

141. T. Verstraelen, D. Van Neck, P. W. Ayers, V. Van Speybroeck and M. Waroquier, Journal of Chemical Theory and Computation, 2007, 3, 1420-1434.

142. R. Bueno-Perez, S. Calero, D. Dubbeldam, C. O. Ania, J. B. Parra, A. P. Zaderenko and P. J. Merkling, Journal of Physical Chemistry C, 2012, 116, 25797-25805.

143. J. B. Nicholas, A. J. Hopfinger, F. R. Trouw and L. E. Iton, Journal of the American Chemical Society, 1991, 113, 4792-4800.

144. P. Demontis, G. B. Suffritti, S. Quartieri, E. S. Fois and A. Gamba, Journal of Physical Chemistry, 1988, 92, 867-871.

145. J. R. Hill and J. Sauer, Journal of Physical Chemistry, 1995, 99, 9536-9550.

146. G. Maurin, R. G. Bell, S. Devautour, F. Henn and J. C. Giuntini, Physical Chemistry Chemical Physics, 2004, 6, 182-187.

147. A. F. Combariza, D. A. Gomez and G. Sastre, Chemical Society Reviews, 2013, 42, 114-127.

148. K. P. Schroder and J. Sauer, Journal of Physical Chemistry, 1996, 100, 11043-11049.

149. A. Pasquarello and R. Car, Physical Review Letters, 1998, 80, 5145-5147.

150. M. Van Houteghem, T. Verstraelen, D. Van Neck, C. Kirschhock, J. A. Martens, M. Waroquier and V. Van Speybroeck, Journal of Chemical Theory and Computation, 2011, 7, 1045-1061.

151. J. F. Haw, ed. Wiley-VCH, 2002.

152. M. Hunger, Wiley-VCH, 2010, vol. 2, p. 105.

153. C. Lamberti, A. Zecchina, E. Groppo and S. Bordiga, Chemical Society Reviews, 2010, 39, 4951-5001.

154. J. A. Lercher and A. Jentys, in. Introduction to Zeolite Science and Practice, ed. Elsevier, 2007, vol. 13.

155. E. Stavitski and B. M. Weckhuysen, Chemical Society Reviews, 2010, 39, 4615-4625.

156. A. Vimont, F. Thibault-Starzyk and M. Daturi, Chemical Society Reviews, 2010, 39, 4928-4950.

157. F. Zaera, Chemical Society Reviews, 2014, 43, 7624-7663.

158. A. Zecchina, G. Spoto and S. Bordiga, Physical Chemistry Chemical Physics, 2005, 7, 16271642.

159. L. Benco, T. Bucko, J. Hafner and H. Toulhoat, Journal of Physical Chemistry B, 2004, 108, 13656-13666.

160. E. G. Derouane, J. C. Vedrine, R. Ramos Pinto, P. M. Borges, L. Costa, M. A. N. D. A. Lemos, F. Lemos and F. Ramoa Ribeiro, Catalysis Reviews-Science and Engineering, 2013, 55, 454-515.

161. J. Sauer, P. Ugliengo, E. Garrone and V. R. Saunders, Chemical Reviews, 1994, 94, 2095-2160.

162. O. Bludsky, P. Nachtigall, P. Cicmamec, P. Knotek and R. Bulanek, Catalysis Today, 2005, 100, 385-389.

163. N. Jardillier, E. A. Villagomez, G. Delahay, B. Coq and D. Berthomieu, Journal of Physical Chemistry B, 2006, 110, 16413-16421.

164. K. Hemelsoet, A. Nollet, V. Van Speybroeck and M. Waroquier, Chemistry-a European Journal, 2011, 17, 9083-9093. 
165. S. Bordiga, L. Regli, D. Cocina, C. Lamberti, M. Bjorgen and K. P. Lillerud, Journal of Physical Chemistry B, 2005, 109, 2779-2784.

166. C. O. Arean, M. R. Delgado, P. Nachtigall, T. Ho Viet, M. Rubes, R. Bulanek and P. ChlubnaEliasova, Physical Chemistry Chemical Physics, 2014, 16, 10129-10141.

167. C. O. Arean, D. Nachtigallova, P. Nachtigall, E. Garrone and M. R. Delgado, Physical Chemistry Chemical Physics, 2007, 9, 1421-1436.

168. C. O. Arean, M. R. Delgado, K. Frolich, R. Bulanek, A. Pulido, G. F. Bibiloni and P. Nachtigall, Journal of Physical Chemistry C, 2008, 112, 4658-4666.

169. O. Bludsky, D. Nachtigallova, R. Bulanek and P. Nachtigall, in Molecular Sieves: From Basic Research to Industrial Applications, Pts $a$ and B, eds. J. Cejka, N. Zilkova and P. Nachtigall, 2005, vol. 158, pp. 625-632.

170. O. Bludsky, M. Silhan, P. Nachtigall, T. Bucko, L. Benco and J. Hafner, Journal of Physical Chemistry B, 2005, 109, 9631-9638.

171. R. Bulanek, P. Cicmanec, P. Knotek, D. Nachtigallova and P. Nachtigall, Physical Chemistry Chemical Physics, 2004, 6, 2003-2007.

172. M. Davidova, D. Nachtigallova, P. Nachtigall and J. Sauer, Journal of Physical Chemistry B, 2004, 108, 13674-13682.

173. P. Nachtigall and R. Bulanek, Applied Catalysis a-General, 2006, 307, 118-127.

174. C. O. Arean, M. R. Delgado, C. L. Bauca, L. Vrbka and P. Nachtigall, Physical Chemistry Chemical Physics, 2007, 9, 4657-4661.

175. D. Nachtigallova, P. Nachtigall and O. Bludsky, Physical Chemistry Chemical Physics, 2004, 6, 5580-5587.

176. R. Bulanek, H. Drobna, P. Nachtigall, M. Rubes and O. Bludsky, Physical Chemistry Chemical Physics, 2006, 8, 5535-5542.

177. R. Bulanek, K. Frolich, P. Cicmanec, D. Nachtigallova, A. Pulido and P. Nachtigall, Journal of Physical Chemistry C, 2011, 115, 13312-13321.

178. R. Bulanek, I. Voleska, E. Ivanova, K. Hadjiivanov and P. Nachtigall, Journal of Physical Chemistry C, 2009, 113, 11066-11076.

179. E. Garrone, R. Bulanek, K. Frolich, C. Otero Arean, M. Rodriguez Delgado, G. Turnes Palomino, D. Nachtigallova and P. Nachtigall, Journal of Physical Chemistry B, 2006, 110, 22542-22550.

180. P. Nachtigall, M. R. Delgado, K. Frolich, R. Bulanek, G. T. Palomino, C. L. Bauca and C. O. Arean, Microporous and Mesoporous Materials, 2007, 106, 162-173.

181. A. Pulido, P. Nachtigall, M. Rodriguez Delgado and C. Otero Arean, Chemphyschem, 2009, 10, 1058-1065.

182. J. P. Merrick, D. Moran and L. Radom, Journal of Physical Chemistry A, 2007, 111, 1168311700.

183. A. P. Scott and L. Radom, Journal of Physical Chemistry, 1996, 100, 16502-16513.

184. O. Bludsky, M. Silhan, D. Nachtigallova and P. Nachtigall, Journal of Physical Chemistry A, 2003, 107, 10381-10388.

185. T. Bucko, J. Hafner and L. Benco, Journal of Physical Chemistry B, 2005, 109, 7345-7357.

186. J. Hafner, L. Benco and T. Bucko, Topics in Catalysis, 2006, 37, 41-54.

187. F. Goeltl and J. Hafner, Journal of Chemical Physics, 2012, 136, 064501.

188. F. Goeltl, R. E. Bulo, J. Hafner and P. Sautet, Journal of Physical Chemistry Letters, 2013, 4, 2244-2249.

189. S. J. Clark, M. D. Segall, C. J. Pickard, P. J. Hasnip, M. J. Probert, K. Refson and M. C. Payne, Zeitschrift Fur Kristallographie, 2005, 220, 567-570.

190. C. J. Pickard and F. Mauri, Physical Review B, 2001, 63, 245101.

191. G. Kresse and J. Furthmuller, Computational Materials Science, 1996, 6, 15-50.

192. G. Kresse and J. Furthmuller, Physical Review B, 1996, 54, 11169-11186. 
193. J. VandeVondele, M. Krack, F. Mohamed, M. Parrinello, T. Chassaing and J. Hutter, Computer Physics Communications, 2005, 167, 103-128.

194. V. Weber, M. Iannuzzi, S. Giani, J. Hutter, R. Declerck and M. Waroquier, Journal of Chemical Physics, 2009, 131, 014106.

195. P. J. Byrne, J. E. Warren, R. E. Morris and S. E. Ashbrook, Solid State Sciences, 2009, 11, 10011006.

196. D. M. Dawson and S. E. Ashbrook, Journal of Physical Chemistry C, 2014, 118, 23285-23296.

197. M. Castro, V. R. Seymour, D. Carnevale, J. M. Griffin, S. E. Ashbrook, P. A. Wright, D. C. Apperley, J. E. Parker, S. P. Thompson, A. Fecant and N. Bats, Journal of Physical Chemistry C, 2010, 114, 12698-12710.

198. A. Depla, D. Lesthaeghe, T. S. van Erp, A. Aerts, K. Houthoofd, F. T. Fan, C. Li, V. Van Speybroeck, M. Waroquier, C. E. A. Kirschhock and J. A. Martens, Journal of Physical Chemistry C, 2011, 115, 3562-3571.

199. A. Depla, E. Verheyen, A. Veyfeyken, M. Van Houteghem, K. Houthoofd, V. Van Speybroeck, M. Waroquier, C. E. A. Kirschhock and J. A. Martens, Journal of Physical Chemistry C, 2011, 115, 11077-11088.

200. A. Zheng, B. Han, B. Li, S.-B. Liu and F. Deng, Chemical Communications, 2012, 48, 69366938.

201. S. Cadars, R. Guegan, M. N. Garaga, X. Bourrat, L. Le Forestier, F. Fayon, H. Tan Vu, T. Allier, Z. Nour and D. Massiot, Chemistry of Materials, 2012, 24, 4376-4389.

202. A. Pulido, G. Sastre and A. Corma, Chemphyschem, 2006, 7, 1092-1099.

203. X. Liu, U. Ravon, F. Bosselet, G. Bergeret and A. Tuel, Chemistry of Materials, 2012, 24, 30163022.

204. K. P. F. Janssen, G. De Cremer, R. K. Neely, A. V. Kubarev, J. Van Loon, J. A. Martens, D. E. De Vos, M. B. J. Roeffaers and J. Hofkens, Chemical Society Reviews, 2014, 43, 990-1006.

205. E. Runge and E. K. U. Gross, Physical Review Letters, 1984, 52, 997-1000.

206. M. A. L. Marques, C. A. Ullrich, F. Nogueira, A. Rubio, K. Burke and E. K. U. Gross, TimeDependent Density Functional Theory, Springer, 2006.

207. C. Adamo and D. Jacquemin, Chemical Society Reviews, 2013, 42, 845-856.

208. V. Barone and A. Polimeno, Chemical Society Reviews, 2007, 36, 1724-1731.

209. T. De Meyer, K. Hemelsoet, L. Van der Schueren, E. Pauwels, K. De Clerck and V. Van Speybroeck, Chemistry-a European Journal, 2012, 18, 8120-8129.

210. A. Baiardi, J. Bloino and V. Barone, Journal of Chemical Theory and Computation, 2013, 9, 4097-4115.

211. V. Barone, J. Bloino, M. Biczysko and F. Santoro, Journal of Chemical Theory and Computation, 2009, 5, 540-554.

212. J. Bloino, M. Biczysko, F. Santoro and V. Barone, Journal of Chemical Theory and Computation, 2010, 6, 1256-1274.

213. F. Santoro, C. Cappelli and V. Barone, Journal of Chemical Theory and Computation, 2011, 7, 1824-1839.

214. V. Van Speybroeck, K. Hemelsoet, K. De Wispelaere, Q. Qian, J. Van der Mynsbrugge, B. De Sterck, B. M. Weckhuysen and M. Waroquier, Chemcatchem, 2013, 5, 173-184.

215. K. Hemelsoet, Q. Qian, T. De Meyer, K. De Wispelaere, B. De Sterck, B. M. Weckhuysen, M. Waroquier and V. Van Speybroeck, Chemistry-a European Journal, 2013, 19, 16595-16606.

216. G. Calzaferri, S. Huber, H. Maas and C. Minkowski, Angewandte Chemie-International Edition, 2003, 42, 3732-3758.

217. E. Fois, G. Tabacchi and G. Calzaferri, Journal of Physical Chemistry C, 2010, 114, $10572-$ 10579.

218. X. Zhou, T. A. Wesolowski, G. Tabacchi, E. Fois, G. Calzaferri and A. Devaux, Physical Chemistry Chemical Physics, 2013, 15, 159-167. 
219. G. Fradelos, J. J. Lutz, T. A. Wesolowski, P. Piecuch and M. Wloch, Journal of Chemical Theory and Computation, 2011, 7, 1647-1666.

220. E. Fois, G. Tabacchi, A. Devaux, P. Belser, D. Bruehwiler and G. Calzaferri, Langmuir, 2013, 29, 9188-9198.

221. A. Pedone, J. Bloino and V. Barone, Journal of Physical Chemistry C, 2012, 116, 17807-17818.

222. D. M. D'Alessandro, B. Smit and J. R. Long, Angewandte Chemie-International Edition, 2010, 49, 6058-6082.

223. M. M. F. Hasan, E. L. First and C. A. Floudas, Physical Chemistry Chemical Physics, 2013, 15, 17601-17618.

224. J. H. Kim, M. AboueInasr, L. C. Lin and B. Smit, Journal of the American Chemical Society, 2013, 135, 7545-7552.

225. R. Krishna and J. M. van Baten, J. Membr. Sci., 2010, 360, 323-333.

226. Z. J. Liang, M. Marshall and A. L. Chaffee, Energy Fuels, 2009, 23, 2785-2789.

227. T. H. Bae, M. R. Hudson, J. A. Mason, W. L. Queen, J. J. Dutton, K. Sumida, K. J. Micklash, S. S. Kaye, C. M. Brown and J. R. Long, Energy Environ. Sci., 2013, 6, 128-138.

228. Y. S. Bae and R. Q. Snurr, Angewandte Chemie-International Edition, 2011, 50, 11586-11596.

229. L. C. Lin, A. H. Berger, R. L. Martin, J. Kim, J. A. Swisher, K. Jariwala, C. H. Rycroft, A. S. Bhown, M. W. Deem, M. Haranczyk and B. Smit, Nature Materials, 2012, 11, 633-641.

230. J. M. Huck, L. C. Lin, A. H. Berger, M. N. Shahrak, R. L. Martin, A. S. Bhown, M. Haranczyk, K. Reuter and B. Smit, Energy Environ. Sci., 2014, 7, 4132-4146.

231. D. Frenkel and B. Smit, Understanding Molecular Simulation, Academic Press, Inc., 2001.

232. J. Kim and B. Smit, Journal of Chemical Theory and Computation, 2012, 8, 2336-2343.

233. D. Dubbeldam, A. Torres-Knoop and K. S. Walton, Mol. Simul., 2013, 39, 1253-1292.

234. C. M. Simon, J. Kim, D. A. Gomez-Gualdron, J. S. Camp, Y. G. Chung, R. L. Martin, R. Mercado, M. W. Deem, D. Gunter, M. Haranczyk, D. S. Sholl, R. Q. Snurr and B. Smit, Energy Environ. Sci., 2015, 8, 1190-1199.

235. A. Goj, D. S. Sholl, E. D. Akten and D. Kohen, Journal of Physical Chemistry B, 2002, 106, 8367-8375.

236. R. L. Martin, B. Smit and M. Haranczyk, J. Chem Inf. Model., 2012, 52, 308-318.

237. R. L. Martin, T. F. Willems, L. C. Lin, J. Kim, J. A. Swisher, B. Smit and M. Haranczyk, Chemphyschem, 2012, 13, 3595-3597.

238. M. Pinheiro, R. L. Martin, C. H. Rycroft, A. Jones, E. Iglesia and M. Haranczyk, Journal of Molecular Graphics \& Modelling, 2013, 44, 208-219.

239. T. F. Willems, C. Rycroft, M. Kazi, J. C. Meza and M. Haranczyk, Microporous and Mesoporous Materials, 2012, 149, 134-141.

240. L. Sarkisov and J. Kim, Chemical Engineering Science, 2015, 121, 322-330.

241. M. Fischer and R. G. Bell, Journal of Physical Chemistry C, 2012, 116, 26449-26463.

242. M. Fischer and R. G. Bell, Journal of Physical Chemistry C, 2013, 117, 17099-17110.

243. H. Fang, P. Kamakoti, J. Zang, S. Cundy, C. Paur, P. I. Ravikovitch and D. S. Sholl, Journal of Physical Chemistry C, 2012, 116, 10692-10701.

244. S. Grimme, Journal of Computational Chemistry, 2006, 27, 1787-1799.

245. H. Fang, P. Kamakoti, P. I. Ravikovitch, M. Aronson, C. Paur and D. S. Sholl, Physical Chemistry Chemical Physics, 2013, 15, 12882-12894.

246. O. Bludsky, M. Rubes, P. Soldan and P. Nachtigall, Journal of Chemical Physics, 2008, 128, 114102.

247. C. Campana, B. Mussard and T. K. Woo, Journal of Chemical Theory and Computation, 2009, 5, 2866-2878.

248. M. Fischer and R. G. Bell, Journal of Physical Chemistry C, 2013, 117, 24446-24454.

249. J. M. Castillo, J. Silvestre-Albero, F. Rodriguez-Reinoso, T. J. H. Vlugt and S. Calero, Physical Chemistry Chemical Physics, 2013, 15, 17374-17382. 
250. N. Desbiens, A. Boutin and I. Demachy, Journal of Physical Chemistry B, 2005, 109, 2407124076.

251. W. Z. Sun, L. C. Lin, X. Peng and B. Smit, Aiche J., 2014, 60, 2314-2323.

252. L. Joos, J. A. Swisher and B. Smit, Langmuir, 2013, 29, 15936-15942.

253. H. J. C. Berendsen, J. R. Grigera and T. P. Straatsma, Journal of Physical Chemistry, 1987, 91, 6269-6271.

254. A. Garcia-Sanchez, C. O. Ania, J. B. Parra, D. Dubbeldam, T. J. H. Vlugt, R. Krishna and S. Calero, Journal of Physical Chemistry C, 2009, 113, 8814-8820.

255. Y. Wang and M. D. Levan, J. Chem. Eng. Data, 2010, 55, 3189-3195.

256. Y. V. Kissin, Catalysis Reviews-Science and Engineering, 2001, 43, 85-146.

257. G. M. Berner and A. L. L. East, Canadian Journal of Chemistry-Revue Canadienne De Chimie, 2009, 87, 1512-1520.

258. C.-J. Chen, S. Rangarajan, I. M. Hill and A. Bhan, Acs Catalysis, 2014, 4, 2319-2327.

259. Q. Li and A. L. L. East, Canadian Journal of Chemistry-Revue Canadienne De Chimie, 2006, 84, 1159-1166.

260. Q. B. Li and A. L. L. East, Canadian Journal of Chemistry-Revue Canadienne De Chimie, 2005, 83, 1146-1157.

261. M. N. Mazar, S. Al-Hashimi, M. Cococcioni and A. Bhan, Journal of Physical Chemistry C, 2013, 117, 23609-23620.

262. N. Rahimi and R. Karimzadeh, Applied Catalysis a-General, 2011, 398, 1-17.

263. J. F. Denayer, G. V. Baron, J. A. Martens and P. A. Jacobs, Journal of Physical Chemistry B, 1998, 102, 3077-3081.

264. J. F. Denayer, W. Souverijns, P. A. Jacobs, J. A. Martens and G. V. Baron, Journal of Physical Chemistry B, 1998, 102, 4588-4597.

265. J. F. M. Denayer and G. V. Baron, Adsorption-Journal of the International Adsorption Society, 1997, 3, 251-265.

266. B. Smit and T. L. M. Maesen, Chemical Reviews, 2008, 108, 4125-4184.

267. K. L. Joshi, S. Raman and A. C. T. van Duin, Journal of Physical Chemistry Letters, 2013, 4, 3792-3797.

268. F. Castro-Marcano and A. C. T. van Duin, Combustion and Flame, 2013, 160, 766-775.

269. M. Boronat, C. M. Zicovich-Wilson, A. Corma and P. Viruela, Physical Chemistry Chemical Physics, 1999, 1, 537-543.

270. M. Boronat, C. M. Zicovich-Wilson, P. Viruela and A. Corma, Chemistry-a European Journal, 2001, 7, 1295-1303.

271. S. R. Blaszkowski and R. A. vanSanten, Journal of the American Chemical Society, 1997, 119, 5020-5027.

272. D. Lesthaeghe, V. Van Speybroeck and M. Waroquier, Journal of the American Chemical Society, 2004, 126, 9162-9163.

273. S. Kasuriya, S. Namuangruk, P. Treesukol, M. Tirtowidjojo and J. Limtrakul, Journal of Catalysis, 2003, 219, 320-328.

274. P. Pantu, B. Boekfa and J. Limtrakul, Journal of Molecular Catalysis a-Chemical, 2007, 277, 171-179.

275. V. Van Speybroeck, J. Van der Mynsbrugge, M. Vandichel, K. Hemelsoet, D. Lesthaeghe, A. Ghysels, G. B. Marin and M. Waroquier, Journal of the American Chemical Society, 2011, 133, 888-899.

276. P. E. Sinclair, A. de Vries, P. Sherwood, C. R. A. Catlow and R. A. van Santen, Journal of the Chemical Society-Faraday Transactions, 1998, 94, 3401-3408.

277. F. Tielens, J. F. M. Denayer, I. Daems, G. V. Baron, W. J. Mortier and P. Geerlings, Journal of Physical Chemistry B, 2003, 107, 11065-11071.

278. M. Vandichel, S. Biswas, K. Leus, J. Paier, J. Sauer, T. Verstraelen, P. Van der Voort, M. Waroquier and V. Van Speybroeck, Chempluschem, 2014, 79, 1183-1197. 
279. V. Van Speybroeck, K. De Wispelaere, J. Van der Mynsbrugge, M. Vandichel, K. Hemelsoet and M. Waroquier, Chemical Society Reviews, 2014, 43, 7326-7357.

280. A. M. Vos, X. Rozanska, R. A. Schoonheydt, R. A. van Santen, F. Hutschka and J. Hafner, Journal of the American Chemical Society, 2001, 123, 2799-2809.

281. S. Grimme, Journal of Computational Chemistry, 2004, 25, 1463-1473.

282. S. Grimme, J. Antony, T. Schwabe and C. Mueck-Lichtenfeld, Organic \& Biomolecular Chemistry, 2007, 5, 741-758.

283. S. Grimme, J. Antony, S. Ehrlich and H. Krieg, Journal of Chemical Physics, 2010, 132, 154104.

284. A. Tkatchenko and M. Scheffler, Physical Review Letters, 2009, 102, 073005.

285. F. Goeltl, A. Grueneis, T. Bucko and J. Hafner, Journal of Chemical Physics, 2012, 137, 114111.

286. R. Y. Brogaard, P. G. Moses and J. K. Norskov, Catalysis Letters, 2012, 142, 1057-1060.

287. B. Boekfa, S. Choomwattana, P. Khongpracha and J. Limtrakul, Langmuir, 2009, 25, 1299012999.

288. J. Van der Mynsbrugge, K. Hemelsoet, M. Vandichel, M. Waroquier and V. Van Speybroeck, Journal of Physical Chemistry C, 2012, 116, 5499-5508.

289. Y. Zhao, N. E. Schultz and D. G. Truhlar, Journal of Chemical Theory and Computation, 2006, 2, 364-382.

290. Y. Zhao and D. G. Truhlar, Journal of Chemical Theory and Computation, 2008, 4, 1849-1868.

291. Y. Zhao and D. G. Truhlar, Accounts of Chemical Research, 2008, 41, 157-167.

292. Y. Zhao and D. G. Truhlar, Journal of Physical Chemistry C, 2008, 112, 6860-6868.

293. J. Gomes, P. M. Zimmerman, M. Head-Gordon and A. T. Bell, Journal of Physical Chemistry C, 2012, 116, 15406-15414.

294. H. Eshuis, J. E. Bates and F. Furche, Theoretical Chemistry Accounts, 2012, 131, 1084.

295. F. Furche, Physical Review B, 2001, 64, 195120.

296. J. Harl and G. Kresse, Physical Review Letters, 2009, 103, 056401.

297. C. Tuma and J. Sauer, Chemical Physics Letters, 2004, 387, 388-394.

298. C. Tuma and J. Sauer, Physical Chemistry Chemical Physics, 2006, 8, 3955-3965.

299. S. Svelle, C. Tuma, X. Rozanska, T. Kerber and J. Sauer, Journal of the American Chemical Society, 2009, 131, 816-825.

300. J. Sauer and M. Sierka, Journal of Computational Chemistry, 2000, 21, 1470-1493.

301. M. Sierka and J. Sauer, Faraday Discussions, 1997, 106, 41-62.

302. B. A. De Moor, M.-F. Reyniers, O. C. Gobin, J. A. Lercher and G. B. Marin, Journal of Physical Chemistry C, 2011, 115, 1204-1219.

303. B. A. De Moor, M.-F. Reyniers and G. B. Marin, Physical Chemistry Chemical Physics, 2009, 11, 2939-2958.

304. B. A. De Moor, M.-F. Reyniers, M. Sierka, J. Sauer and G. B. Marin, Journal of Physical Chemistry C, 2008, 112, 11796-11812.

305. C. M. Nguyen, B. A. De Moor, M.-F. Reyniers and G. B. Marin, Journal of Physical Chemistry C, 2011, 115, 23831-23847.

306. F. Eder and J. A. Lercher, Zeolites, 1997, 18, 75-81.

307. C.-C. Chiu, G. N. Vayssilov, A. Genest, A. Borgna and N. Roesch, Journal of Computational Chemistry, 2014, 35, 809-819.

308. K. Lee, E. D. Murray, L. Kong, B. I. Lundqvist and D. C. Langreth, Physical Review B, 2010, 82, 081101.

309. B. A. De Moor, M. F. Reyniers, O. C. Gobin, J. A. Lercher and G. B. Marin, Journal of Physical Chemistry C, 2011, 115, 1204-1219.

310. D. C. Tranca, N. Hansen, J. A. Swisher, B. Smit and F. J. Keil, Journal of Physical Chemistry C, 2012, 116, 23408-23417.

311. A. Ghysels, T. Verstraelen, K. Hemelsoet, M. Waroquier and V. Van Speybroeck, J. Chem Inf. Model., 2010, 50, 1736-1750. 
312. A. Ghysels, D. Van Neck, V. Van Speybroeck, T. Verstraelen and M. Waroquier, Journal of Chemical Physics, 2007, 126, 224102.

313. J. Van der Mynsbrugge, S. L. C. Moors, K. De Wispelaere and V. Van Speybroeck, Chemcatchem, 2014, 6, 1906-1918.

314. D. Rutenbeck, H. Papp, H. Ernst and W. Schwieger, Applied Catalysis a-General, 2001, 208, 153-161.

315. V. Nieminen, M. Sierka, D. Y. Murzin and J. Sauer, Journal of Catalysis, 2005, 231, 393-404.

316. C. Tuma and J. Sauer, Angewandte Chemie-International Edition, 2005, 44, 4769-4771.

317. S. Schallmoser, J. Van der Mynsbrugge, K. De Wispelaere, M. Waroquier, G. L. Haller, M. Sanchez-Sanchez, V. Van Speybroeck and J. A. Lercher, Journal of Physical Chemistry C, 2015, submitted.

318. S. W. Yang, J. N. Kondo and K. Domen, Journal of Physical Chemistry B, 2001, 105, 78787881.

319. S. W. Yang, J. N. Kondo and K. Domen, Catalysis Today, 2002, 73, 113-125.

320. J. F. Haw, Physical Chemistry Chemical Physics, 2002, 4, 5431-5441.

321. M. Bjorgen, F. Bonino, S. Kolboe, K. P. Lillerud, A. Zecchina and S. Bordiga, Journal of the American Chemical Society, 2003, 125, 15863-15868.

322. L. A. Clark, M. Sierka and J. Sauer, Journal of the American Chemical Society, 2004, 126, 936947.

323. L. A. Clark, M. Sierka and J. Sauer, Journal of the American Chemical Society, 2003, 125, 2136-2141.

324. M. Boronat and A. Corma, Applied Catalysis a-General, 2008, 336, 2-10.

325. C. Tuma, T. Kerber and J. Sauer, Angewandte Chemie-International Edition, 2010, 49, 46784680.

326. W. Wang and M. Hunger, Accounts of Chemical Research, 2008, 41, 895-904.

327. J. B. Nicholas and J. F. Haw, Journal of the American Chemical Society, 1998, 120, 1180411805.

328. V. Termath, F. Haase, J. Sauer, J. Hutter and M. Parrinello, Journal of the American Chemical Society, 1998, 120, 8512-8516.

329. H. Fang, A. Zheng, J. Xu, S. Li, Y. Chu, L. Chen and F. Deng, Journal of Physical Chemistry C, 2011, 115, 7429-7439.

330. J. F. Haw, J. B. Nicholas, W. G. Song, F. Deng, Z. K. Wang, T. Xu and C. S. Heneghan, Journal of the American Chemical Society, 2000, 122, 4763-4775.

331. A. G. Stepanov, V. N. Sidelnikov and K. I. Zamaraev, Chemistry-a European Journal, 1996, 2, 157-167.

332. F. G. Oliver, E. J. Munson and J. F. Haw, Journal of Physical Chemistry, 1992, 96, 8106-8111.

333. T. Xu and J. F. Haw, Journal of the American Chemical Society, 1994, 116, 10188-10195.

334. J. F. Haw, B. R. Richardson, I. S. Oshiro, N. D. Lazo and J. A. Speed, Journal of the American Chemical Society, 1989, 111, 2052-2058.

335. W. G. Song, J. B. Nicholas, A. Sassi and J. F. Haw, Catalysis Letters, 2002, 81, 49-53.

336. C. Wattanakit, S. Nokbin, B. Boekfa, P. Pantu and J. Limtrakul, Journal of Physical Chemistry C, 2012, 116, 5654-5663.

337. M. Boronat, P. Viruela and A. Corma, Physical Chemistry Chemical Physics, 2001, 3, 32353239.

338. D. Gleeson, Journal of Physical Chemistry A, 2011, 115, 14629-14636.

339. F. Ferrante, T. Rubino and D. Duca, Journal of Physical Chemistry C, 2011, 115, 14862-14868.

340. N. D. Lazo, B. R. Richardson, P. D. Schettler, J. L. White, E. J. Munson and J. F. Haw, Journal of Physical Chemistry, 1991, 95, 9420-9425.

341. H. Ishikawa, E. Yoda, J. N. Kondo, F. Wakabayashi and K. Domen, Journal of Physical Chemistry B, 1999, 103, 5681-5686.

342. A. G. Stepanov, Kinetics and Catalysis, 2010, 51, 854-872. 
343. P. Tiwary and M. Parrinello, Physical Review Letters, 2013, 111, 230602.

344. F. C. Jentoft and B. C. Gates, Topics in Catalysis, 1997, 4, 1-13.

345. S. Kotrel, H. Knozinger and B. C. Gates, Microporous and Mesoporous Materials, 2000, 35-6, 11-20.

346. J. Weitkamp, Chemcatchem, 2012, 4, 292-306.

347. W. O. Haag and R. M. Dessau, Proceedings of the eight international congress on catalysis, Dechema : Frankfurt am Main, 1984.

348. T. F. Narbeshuber, H. Vinek and J. A. Lercher, Journal of Catalysis, 1995, 157, 388-395.

349. J. Wei, Chemical Engineering Science, 1996, 51, 2995-2999.

350. C. E. Ramachandran, B. A. Williams, J. A. van Bokhoven and J. T. Miller, Journal of Catalysis, 2005, 233, 100-108.

351. S. M. Babitz, B. A. Williams, J. T. Miller, R. Q. Snurr, W. O. Haag and H. H. Kung, Applied Catalysis a-General, 1999, 179, 71-86.

352. J. A. van Bokhoven, B. A. Williams, W. Ji, D. C. Koningsberger, H. H. Kung and J. T. Miller, Journal of Catalysis, 2004, 224, 50-59.

353. A. Bhan, R. Gounder, J. Macht and E. Iglesia, Journal of Catalysis, 2008, 253, 221-224.

354. R. Gounder and E. Iglesia, Journal of the American Chemical Society, 2009, 131, 1958-1971.

355. S. R. Blaszkowski, M. A. C. Nascimento and R. A. vanSanten, Journal of Physical Chemistry, 1996, 100, 3463-3472.

356. S. R. Blaszkowski and R. A. vanSanten, Topics in Catalysis, 1997, 4, 145-156.

357. M. Boronat, P. Viruela and A. Corma, Physical Chemistry Chemical Physics, 2000, 2, $3327-$ 3333.

358. A. M. Rigby, G. J. Kramer and R. A. vanSanten, Journal of Catalysis, 1997, 170, 1-10.

359. X. B. Zheng and P. Blowers, Journal of Physical Chemistry A, 2005, 109, 10734-10741.

360. P. M. Zimmerman, M. Head-Gordon and A. T. Bell, Journal of Chemical Theory and Computation, 2011, 7, 1695-1703.

361. J. Lomratsiri, M. Probst and J. Limtrakul, Journal of Molecular Graphics \& Modelling, 2006, 25, 219-225.

362. A. M. Shor, E. A. I. Shor, S. Laletina, V. A. Nasluzov, G. N. Vayssilov and N. Roesch, Chemical Physics, 2009, 363, 33-41.

363. L. Benco, T. Bucko and J. Hafner, Journal of Physical Chemistry C, 2009, 113, 18807-18816.

364. X. Solans-Monfort, M. Sodupe and J. Eckert, Journal of Physical Chemistry C, 2010, 114, 13926-13934.

365. G. Li, E. A. Pidko, R. A. van Santen, Z. Feng, C. Li and E. J. M. Hensen, Journal of Catalysis, 2011, 284, 194-206.

366. F. Goeltl and J. Hafner, Microporous and Mesoporous Materials, 2013, 166, 176-184.

367. N. Hansen and F. J. Keil, Chemie Ingenieur Technik, 2013, 85, 413-419.

368. F. J. Keil, Computers \& Mathematics with Applications, 2013, 65, 1674-1697.

369. J. A. Swisher, N. Hansen, T. Maesen, F. J. Keil, B. Smit and A. T. Bell, Journal of Physical Chemistry C, 2010, 114, 10229-10239.

370. T. Jiang, F. Goeltl, R. E. Bulo and P. Sautet, Acs Catalysis, 2014, 4, 2351-2358.

371. P. M. Zimmerman, D. C. Tranca, J. Gomes, D. S. Lambrecht, M. Head-Gordon and A. T. Bell, Journal of the American Chemical Society, 2012, 134, 19468-19476.

372. T. Bucko, L. Benco, J. Hafner and J. G. Angyan, Journal of Catalysis, 2011, 279, 220-228.

373. T. Bucko and J. Hafner, Journal of Physics-Condensed Matter, 2010, 22, 384201.

374. P. G. Bolhuis, D. Chandler, C. Dellago and P. L. Geissler, Annual Review of Physical Chemistry, 2002, 53, 291-318.

375. J. J. P. Stewart, L. P. Davis and L. W. Burggraf, Journal of Computational Chemistry, 1987, 8, 1117-1123.

376. S. M. Sharada, P. M. Zimmerman, A. T. Bell and M. Head-Gordon, Journal of Physical Chemistry C, 2013, 117, 12600-12611. 
377. T. Bucko, Journal of Physics-Condensed Matter, 2008, 20, 064211.

378. P. Fleurat-Lessard and T. Ziegler, Journal of Chemical Physics, 2005, 123, 084101.

379. B. M. Dickson, F. Legoll, T. Lelievre, G. Stoltz and P. Fleurat-Lessard, Journal of Physical Chemistry B, 2010, 114, 5823-5830.

380. C. Dellago, P. G. Bolhuis and P. L. Geissler, Advances in Chemical Physics, Vol 123, 2002, 123, 1-78.

381. B. Xu, C. Sievers, S. B. Hong, R. Prins and J. A. van Bokhoven, Journal of Catalysis, 2006, 244, 163-168.

382. C. D. Chang, Catalysis Reviews - Science and Engineering, 1983, 25, 1-118.

383. C. D. Chang, Catalysis Today, 1992, 13, 103-111.

384. U. Olsbye, S. Svelle, M. Bjorgen, P. Beato, T. V. W. Janssens, F. Joensen, S. Bordiga and K. P. Lillerud, Angewandte Chemie-International Edition, 2012, 51, 5810-5831.

385. , 2013.

386. W. G. Song, D. M. Marcus, H. Fu, J. O. Ehresmann and J. F. Haw, Journal of the American Chemical Society, 2002, 124, 3844-3845.

387. M. Stocker, Microporous and Mesoporous Materials, 1999, 29, 3-48.

388. I. M. Dahl and S. Kolboe, Catalysis Letters, 1993, 20, 329-336.

389. I. M. Dahl and S. Kolboe, Journal of Catalysis, 1994, 149, 458-464.

390. I. M. Dahl and S. Kolboe, Journal of Catalysis, 1996, 161, 304-309.

391. J. F. Haw, W. G. Song, D. M. Marcus and J. B. Nicholas, Accounts of Chemical Research, 2003, 36, 317-326.

392. D. Lesthaeghe, V. Van Speybroeck, G. B. Marin and M. Waroquier, Angewandte ChemieInternational Edition, 2006, 45, 1714-1719.

393. D. Lesthaeghe, V. Van Speybroeck, G. B. Marin and M. Waroquier, Industrial \& Engineering Chemistry Research, 2007, 46, 8832-8838.

394. K. Hemelsoet, J. Van der Mynsbrugge, K. De Wispelaere, M. Waroquier and V. Van Speybroeck, Chemphyschem, 2013, 14, 1526-1545.

395. I. M. Hill, S. Al Hashimi and A. Bhan, Journal of Catalysis, 2012, 285, 115-123.

396. Saepurahman, M. Visur, U. Olsbye, M. Bjorgen and S. Svelle, Topics in Catalysis, 2011, 54, 1293-1301.

397. S. Svelle, P. A. Ronning and S. Kolboe, Journal of Catalysis, 2004, 224, 115-123.

398. S. Svelle, P. O. Ronning, U. Olsbye and S. Kolboe, Journal of Catalysis, 2005, 234, 385-400.

399. J. Van der Mynsbrugge, M. Visur, U. Olsbye, P. Beato, M. Bjorgen, V. Van Speybroeck and S. Svelle, Journal of Catalysis, 2012, 292, 201-212.

400. R. Y. Brogaard, R. Henry, Y. Schuurman, A. J. Medford, P. G. Moses, P. Beato, S. Svelle, J. K. Norskov and U. Olsbye, Journal of Catalysis, 2014, 314, 159-169.

401. A. J. Jones and E. Iglesia, Angewandte Chemie-International Edition, 2014, 53, 12177-12181.

402. J. Van der Mynsbrugge, PhD, Ghent University, 2014.

403. R. Gounder and E. Iglesia, Accounts of Chemical Research, 2012, 45, 229-238.

404. J. Van der Mynsbrugge, J. De Ridder, K. Hemelsoet, M. Waroquier and V. Van Speybroeck, Chemistry-a European Journal, 2013, 19, 11568-11576.

405. C.-M. Wang, R. Y. Brogaard, B. M. Weckhuysen, J. K. Norskov and F. Studt, Journal of Physical Chemistry Letters, 2014, 5, 1516-1521.

406. L. Benco, T. Bucko and J. Hafner, Journal of Catalysis, 2011, 277, 104-116.

407. T. Bucko, L. Benco, O. Dubay, C. Dellago and J. Hafner, Journal of Chemical Physics, 2009, 131, 214508.

408. T. Bucko, L. Benco, J. Hafner and J. G. Angyan, Journal of Catalysis, 2007, 250, 171-183.

409. M. W. Erichsen, K. De Wispelaere, K. Hemelsoet, S. L. C. Moors, T. De Coninck, M. Waroquier, S. Svelle, V. Van Speybroeck and U. Olsbye, Journal of Catalysis, 2015.

410. J. Gomes, M. Head-Gordon and A. T. Bell, Journal of Physical Chemistry C, 2014, 118, 2140921419. 
411. J. Limtrakul, P. Chuichay and S. Nokbin, Journal of Molecular Structure, 2001, 560, 169-177.

412. J. Kanellopoulos, C. Gottert, D. Schneider, B. Knorr, D. Prager, H. Ernst and D. Freude, Journal of Catalysis, 2008, 255, 68-78.

413. J. A. Ryder, A. K. Chakraborty and A. T. Bell, Journal of Physical Chemistry B, 2000, 104, 69987011.

414. I. Stich, J. D. Gale, K. Terakura and M. C. Payne, Chemical Physics Letters, 1998, 283, 402-408.

415. S. L. C. Moors, K. De Wispelaere, J. Van der Mynsbrugge, M. Waroquier and V. Van Speybroeck, Acs Catalysis, 2013, 3, 2556-2567.

416. A. Laio and M. Parrinello, Proc. Natl. Acad. Sci. U. S. A., 2002, 99, 12562-12566.

417. K. De Wispelaere, K. Hemelsoet, M. Waroquier and V. Van Speybroeck, Journal of Catalysis, 2013, 305, 76-80.

418. D. M. McCann, D. Lesthaeghe, P. W. Kletnieks, D. R. Guenther, M. J. Hayman, V. Van Speybroeck, M. Waroquier and J. F. Haw, Angewandte Chemie-International Edition, 2008, 47, 5179-5182.

419. D. Lesthaeghe, A. Horre, M. Waroquier, G. B. Marin and V. Van Speybroeck, Chemistry- $a$ European Journal, 2009, 15, 10803-10808.

420. S. Svelle, F. Joensen, J. Nerlov, U. Olsbye, K. P. Lillerud, S. Kolboe and M. Bjorgen, Journal of the American Chemical Society, 2006, 128, 14770-14771.

421. C. M. Wang, Y. D. Wang, Z. K. Xie and Z. P. Liu, Journal of Physical Chemistry C, 2009, 113, 4584-4591.

422. S. Xu, A. Zheng, Y. Wei, J. Chen, J. Li, Y. Chu, M. Zhang, Q. Wang, Y. Zhou, J. Wang, F. Deng and Z. Liu, Angewandte Chemie-International Edition, 2013, 52, 11564-11568.

423. B. P. C. Hereijgers, F. Bleken, M. H. Nilsen, S. Svelle, K. P. Lillerud, M. Bjorgen, B. M. Weckhuysen and U. Olsbye, Journal of Catalysis, 2009, 264, 77-87.

424. W. Dai, M. Scheibe, L. Li, N. Guan and M. Hunger, Journal of Physical Chemistry C, 2012, 116, 2469-2476.

425. W. Dai, G. Wu, L. Li, N. Guan and M. Hunger, Acs Catalysis, 2013, 3, 588-596.

426. K. Hemelsoet, A. Nollet, M. Vandichel, D. Lesthaeghe, V. Van Speybroeck and M. Waroquier, Chemcatchem, 2009, 1, 373-378.

427. P. Concepcion, A. Corma, J. M. L. Nieto and J. PerezPariente, Applied Catalysis a-General, 1996, 143, 17-28.

428. J. M. Thomas, R. Raja, G. Sankar and R. G. Bell, Nature, 1999, 398, 227-230.

429. J. M. Thomas, R. Raja, G. Sankar and R. G. Bell, Accounts of Chemical Research, 2001, 34, 191-200.

430. D. L. Vanoppen, D. E. Devos, M. J. Genet, P. G. Rouxhet and P. A. Jacobs, Angewandte Chemie-International Edition in English, 1995, 34, 560-563.

431. F. Cora, L. Gomez-Hortigueela and C. R. A. Catlow, Proceedings of the Royal Society aMathematical Physical and Engineering Sciences, 2012, 468, 2053-2069.

432. L. Gomez-Hortigueela, F. Cora and C. R. A. Catlow, Acs Catalysis, 2011, 1, 1487-1497.

433. L. Gomez-Hortigueela, F. Cora and C. R. A. Catlow, Acs Catalysis, 2011, 1, 945-955.

434. L. Gomez-Hortigueela, F. Cora and C. R. A. Catlow, Acs Catalysis, 2011, 1, 18-28.

435. L. Gomez-Hortigueela, F. Cora and C. R. A. Catlow, Journal of Physical Chemistry C, 2012, 116, 6691-6702.

436. L. Gomez-Hortigueela, F. Cora and C. R. A. Catlow, Physical Chemistry Chemical Physics, 2013, 15, 6870-6874.

437. B. Notari, in Advances in Catalysis, Vol 41, eds. D. D. Eley, W. O. Haag and B. Gates, 1996, vol. 41, pp. 253-334.

438. T. Maschmeyer, F. Rey, G. Sankar and J. M. Thomas, Nature, 1995, 378, 159-162.

439. G. Sankar, F. Rey, J. M. Thomas, G. N. Greaves, A. Corma, B. R. Dobson and A. J. Dent, Journal of the Chemical Society-Chemical Communications, 1994, 2279-2280. 
440. J. To, A. A. Sokol, S. A. French and C. R. A. Catlow, Journal of Physical Chemistry C, 2007, 111, 14720-14731.

441. J. To, A. A. Sokol, S. A. French, C. R. A. Catlow, P. Sherwood and H. J. J. van Dam, Angewandte Chemie-International Edition, 2006, 45, 1633-1638.

442. ChemShell, A Computational Chemistry Shell, www.chemshell.org.

443. S. Metz, J. Kaestner, A. A. Sokol, T. W. Keal and P. Sherwood, Wiley Interdisciplinary ReviewsComputational Molecular Science, 2014, 4, 101-110.

444. P. Sherwood, A. H. de Vries, M. F. Guest, G. Schreckenbach, C. R. A. Catlow, S. A. French, A. A. Sokol, S. T. Bromley, W. Thiel, A. J. Turner, S. Billeter, F. Terstegen, S. Thiel, J. Kendrick, S. C. Rogers, J. Casci, M. Watson, F. King, E. Karlsen, M. Sjovoll, A. Fahmi, A. Schafer and C. Lennartz, Journal of Molecular Structure-Theochem, 2003, 632, 1-28.

445. F. Cora, M. Alfredsson, C. M. Barker, R. G. Bell, M. D. Foster, I. Saadoune, A. Simperler and C. R. A. Catlow, Journal of Solid State Chemistry, 2003, 176, 496-529.

446. C. M. Barker, D. Gleeson, N. Kaltsoyannis, C. R. A. Catlow, G. Sankar and J. M. Thomas, Physical Chemistry Chemical Physics, 2002, 4, 1228-1240.

447. D. Berger, A. J. Logsdail, H. Oberhofer, M. R. Farrow, C. R. A. Catlow, P. Sherwood, A. A. Sokol, V. Blum and K. Reuter, Journal of Chemical Physics, 2014, 141, 024105.

448. S. Brandenberger, O. Kroecher, A. Tissler and R. Althoff, Catalysis Reviews-Science and Engineering, 2008, 50, 492-531.

449. S. T. Korhonen, D. W. Fickel, R. F. Lobo, B. M. Weckhuysen and A. M. Beale, Chemical Communications, 2011, 47, 800-802.

450. F. Gao, J. H. Kwak, J. Szanyi and C. H. F. Peden, Topics in Catalysis, 2013, 56, 1441-1459.

451. U. Deka, I. Lezcano-Gonzalez, B. M. Weckhuysen and A. M. Beale, Acs Catalysis, 2013, 3, 413427.

452. J. H. Kwak, T. Varga, C. H. F. Peden, F. Gao, J. C. Hanson and J. Szanyi, Journal of Catalysis, 2014, 314, 83-93.

453. J. S. McEwen, T. Anggara, W. F. Schneider, V. F. Kispersky, J. T. Miller, W. N. Delgass and F. H. Ribeiro, Catalysis Today, 2012, 184, 129-144.

454. C. W. Andersen, M. Bremholm, P. N. Vennestrom, A. B. Blichfeld, L. F. Lundegaard and B. B. Iversen, IUCrJ, 2014, 1, 382-386.

455. P. Vanelderen, J. Vancauwenbergh, B. F. Sels and R. A. Schoonheydt, Coordination Chemistry Reviews, 2013, 257, 483-494.

456. U. Deka, A. Juhin, E. A. Eilertsen, H. Emerich, M. A. Green, S. T. Korhonen, B. M. Weckhuysen and A. M. Beale, Journal of Physical Chemistry C, 2012, 116, 4809-4818.

457. F. Giordanino, E. Borfecchia, K. A. Lomachenko, A. Lazzarini, G. Agostini, E. Gallo, A. V. Soldatov, P. Beato, S. Bordiga and C. Lamberti, Journal of Physical Chemistry Letters, 2014, 5, 1552-1559.

458. E. Borfecchia, K. A. Lomachenko, F. Giordanino, H. Falsig, P. Beato, A. V. Soldatov, S. Bordiga and C. Lamberti, Chemical Science, 2015, 6, 548-563.

459. I. Lezcano-Gonzalez, U. Deka, B. Arstad, A. Van Yperen-De Deyne, K. Hemelsoet, M. Waroquier, V. Van Speybroeck, B. M. Weckhuysen and A. M. Beale, Physical Chemistry Chemical Physics, 2014, 16, 1639-1650.

460. A. F. Combariza, G. Sastre and A. Corma, Journal of Physical Chemistry C, 2011, 115, 875-884.

461. M. P. Allen and D. J. Tildesley, Computer simulation of liquids, Clarendon Press, 1989.

462. I. T. Todorov, W. Smith, K. Trachenko and M. T. Dove, Journal of Materials Chemistry, 2006, 16, 1911-1918.

463. M. K. F. Abouelnasr and B. Smit, Physical Chemistry Chemical Physics, 2012, 14, 11600 11609.

464. A. J. O'Malley and A. Catlow, Physical Chemistry Chemical Physics, 2015, 17, 1943 - 1948.

465. H. Jobic, Journal of Molecular Catalysis a-Chemical, 2000, 158, 135-142.

466. H. Jobic and D. N. Theodorou, Journal of Physical Chemistry B, 2006, 110, 1964-1967. 
467. P. K. Ghorai, S. Yashonath, P. Demontis and G. B. Suffritti, Journal of the American Chemical Society, 2003, 125, 7116-7123.

468. R. C. Runnebaum and E. J. Maginn, Journal of Physical Chemistry B, 1997, 101, 6394-6408.

469. A. J. O'Malley and C. R. A. Catlow, Physical Chemistry Chemical Physics, 2013, 15, 1902419030.

470. B. Smit and R. Krishna, Chemical Engineering Science, 2003, 58, 557-568.

471. R. V. Awati, P. I. Ravikovitch and D. S. Sholl, Journal of Physical Chemistry C, 2013, 117, 13462-13473.

472. H. Fang, H. Demir, P. Kamakoti and D. S. Sholl, Journal of Materials Chemistry A, 2014, 2, 274-291.

473. C. Wang, B. Li, Y. Wang and Z. Xie, Journal of Energy Chemistry, 2013, 22, 914-918.

474. G. Sastre, Catalysis Today, 2014, 226, 25-36.

475. D. Kubicka, I. Kubickova and J. Cejka, Catalysis Reviews-Science and Engineering, 2013, 55, 178. 